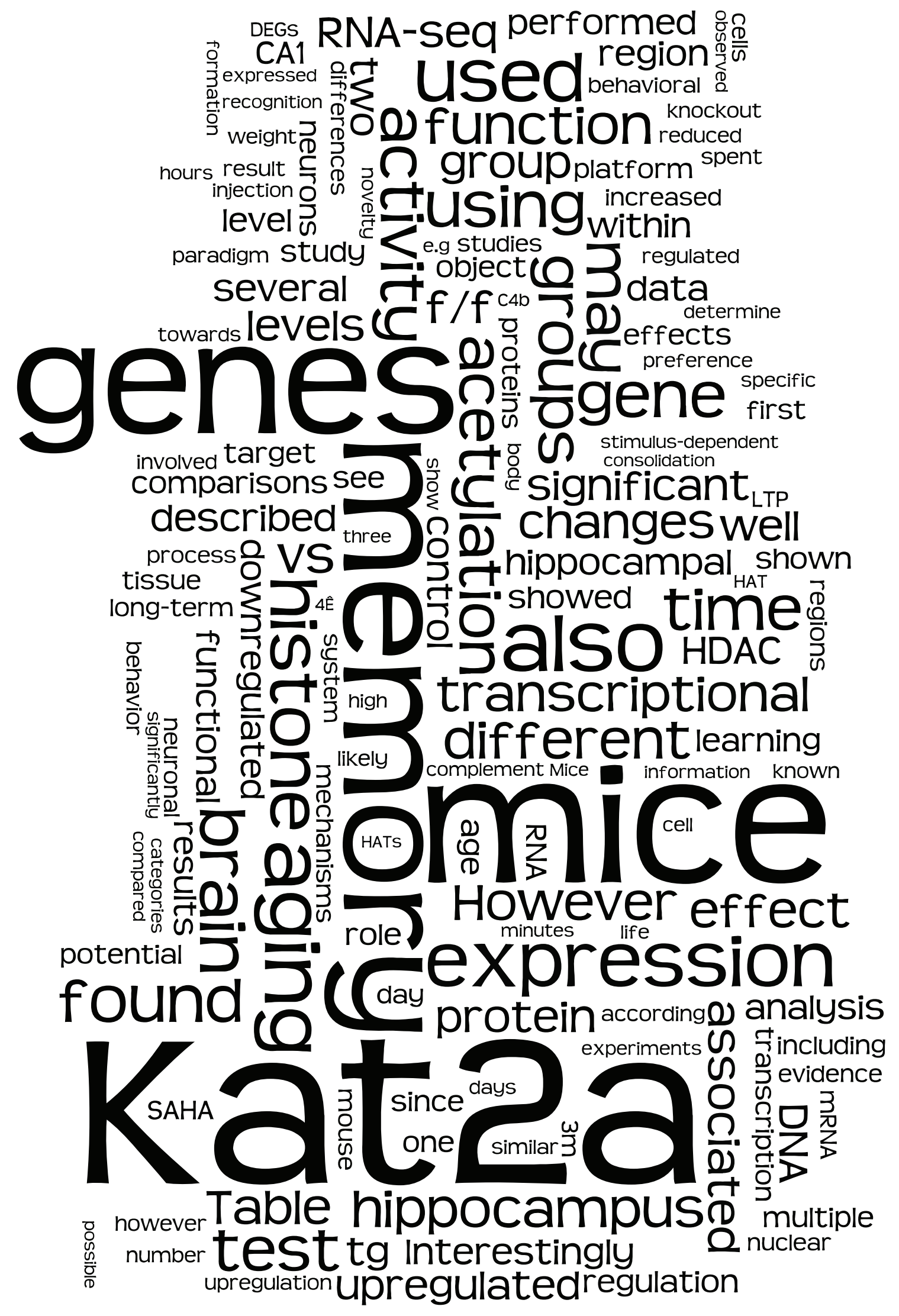




\section{The role of Kat2a during memory formation and chromatin plasticity in the aging murine hippocampus}

Dissertation

in partial fulfillment of the requirements

for the degree "Doctor rerum naturalium"

in the Neuroscience Program

at the Georg-August-University Göttingen, Faculty of Biology

submitted by

Roman Manuel Stilling

born in

Beja / Portugal

Göttingen 2013 


\section{PhD Thesis Committee:}

1. Prof. Dr. André Fischer: Deutsches Zentrum für Neurodegenerative Erkrankungen (DZNE), University Medical Center, Göttingen (GUTACHTER)

2. Prof. Dr. André Fiala: Johann-Friedrich-Blumenbach Institute for Zoologie und Anthropologie, Schwann-Schleiden Forschungszentrum, Georg-AugustUniversität, Göttingen (GUTACHTER)

3. Dr. Judith Stegmüller: Max-Planck-Institut für Experimentelle Medizin, Göttingen

Date of Oral Examination: $\quad 19.04 .2013$

\section{Extended Thesis Committee:}

- Prof. Dr. Michael Hörner: Johann-Friedrich-Blumenbach Institute for Zoologie und Anthropologie and IMPRS Neurosciences, Georg-August-Universität, Göttingen

- Dr. Camin Dean: European Neuroscience Institute Göttingen (ENI-G), University Medical Center and Max-Planck Society, Göttingen

- Prof. Dr. Gregor Eichele: Max-Planck-Institut für Biophysikalische Chemie, Göttingen 


\section{Declaration}

I herewith declare that I have prepared the dissertation "The role of Kat2a during memory formation and chromatin plasticity in the aging murine hippocampus" entirely by myself with no other aids or sources than quoted. 


\section{Table of Contents}

Table of Contents

Acknowledgements

List of Abbreviations ...

List of Figures.. IX

List of Tables

Preface XII

Summary XIII

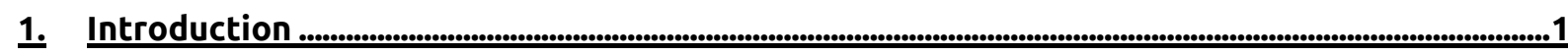

1.1. Learning and Memory ............................................................................................................... 1

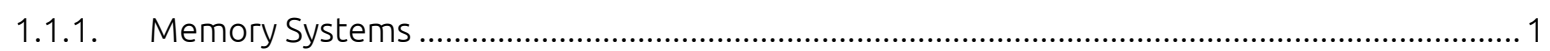

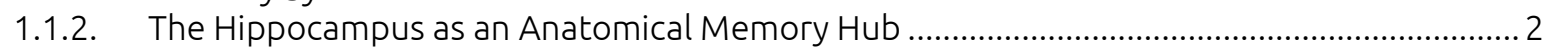

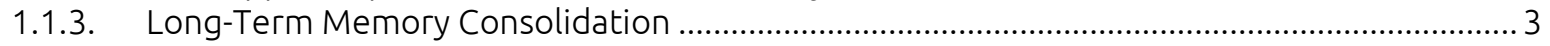

1.1.3.1. Protein Synthesis and Transcription .....................................................................................................

1.1.3.2. Long-Term Potentiation and the Synaptic Tagging and Capture Hypothesis .......................................

1.1.4. The mouse as a model organism for the study of learning and memory ............................... 5

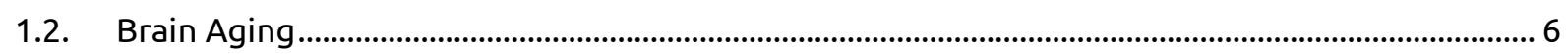

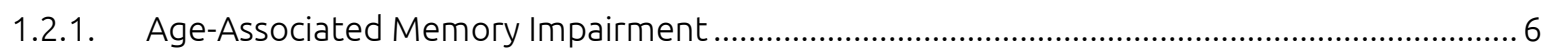

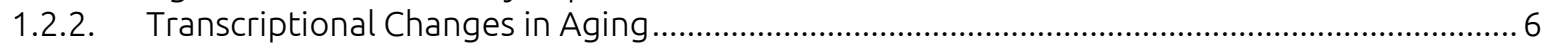

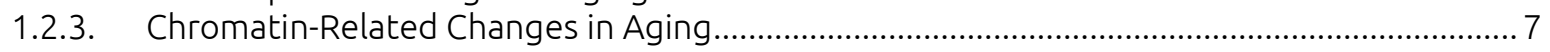

1.3. Chromatin Plasticity and Regulation of Transcription ......................................................................... 8

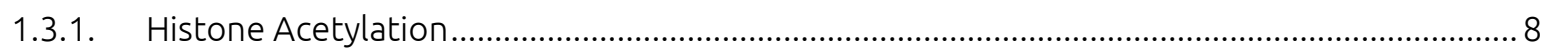

1.3.1.1. Histone acetylation in learning and memory ............................................................................................9

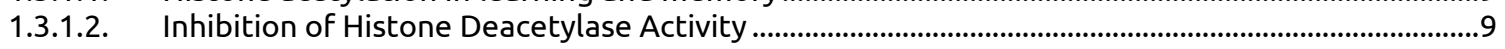

1.3.2. Stimulus-Dependent Regulation of Gene Expression ............................................................10

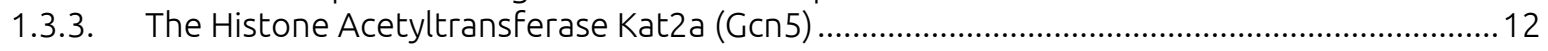

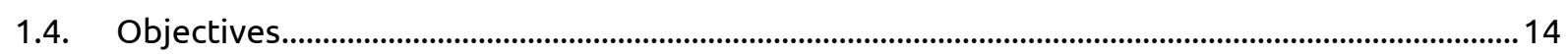

1.4.1. The role of Kat2a in transcription during learning and memory ...........................................14

1.4.2. Histone acetylation and transcription in the aging hippocampus ..........................................14

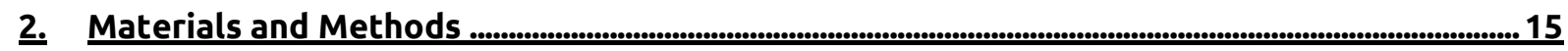

2.1. Animals and Tissue Isolation .................................................................................................15

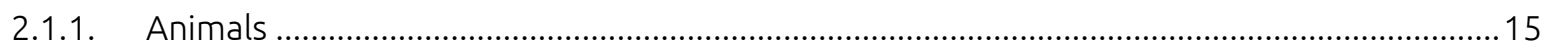

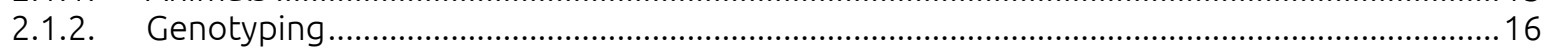

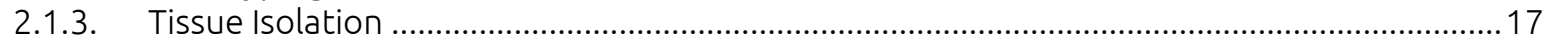

2.1.3.1. Tissue Isolation for Extraction of Biological Macromolecules ............................................................. 17

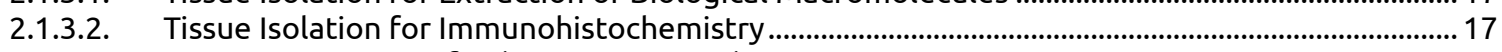

2.1.4. Stereotaxic injection of Adeno-associated virus .................................................................17

2.2. Pharmacological Inhibition of HDAC Activity ............................................................................... 18

2.2.1. Injection of Suberoylanilide Hydroxamic Acid in Young Mice .................................................. 18

2.2.2. Oral Administration of Suberoylanilide Hydroxamic Acid in Aged Mice ................................18 
2.3. Behavioral Analyses...

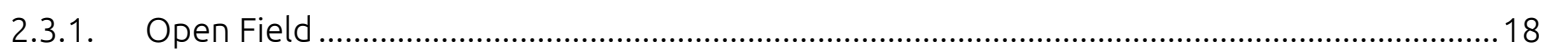

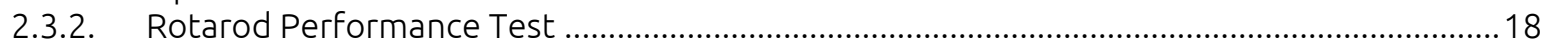

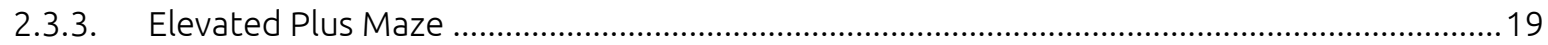

2.3.4. 4-armed Cross Maze-Exploration Test...................................................................................19

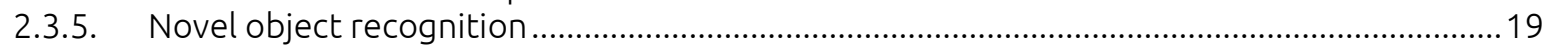

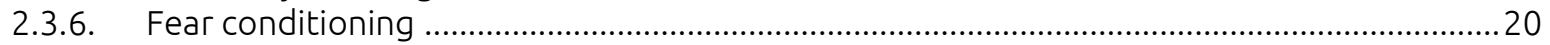

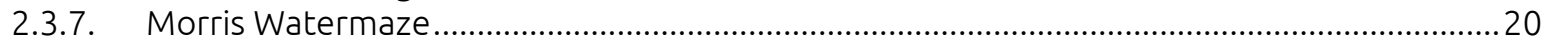

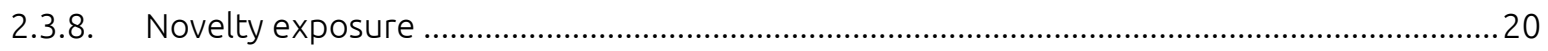

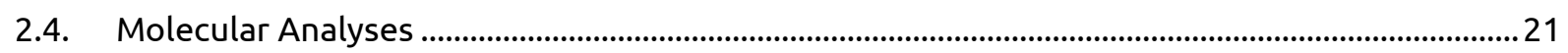

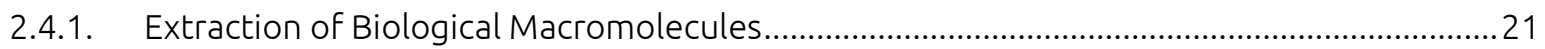

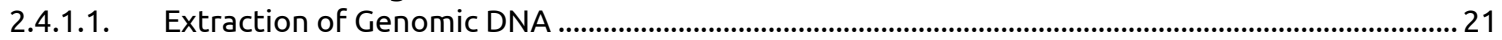

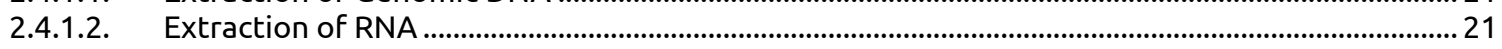

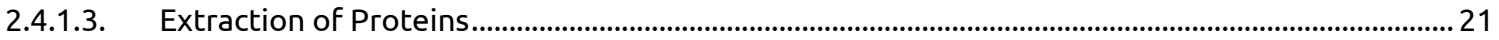

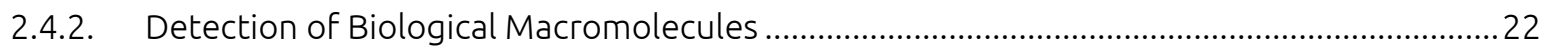

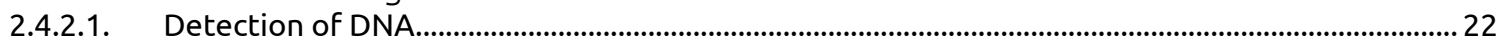

2.4.2.1.1. DNA Quantity Measurements and Quality Control .....................................................................22

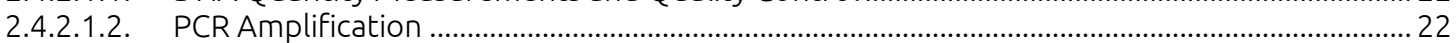

2.4.2.1.3. DNA Gel Electrophoresis and Purification ...................................................................................2 22

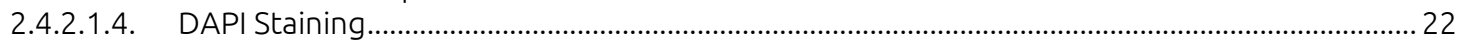

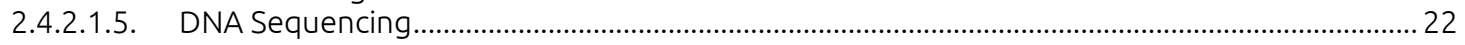

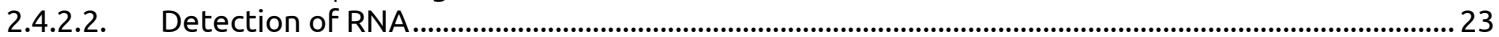

2.4.2.2.1. RNA Quantity Measurements and Quality Control ....................................................................2 23

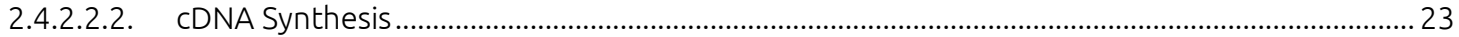

2.4.2.2.3. GRT-PCR

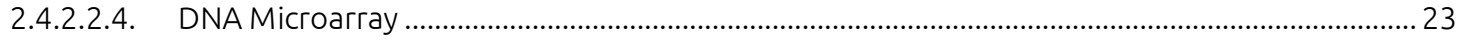

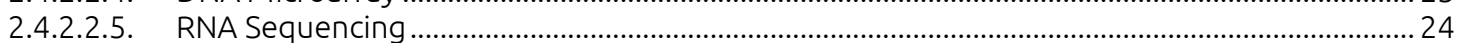

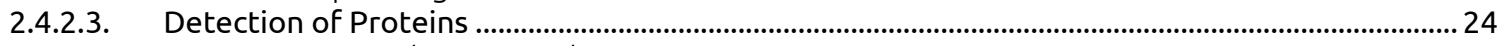

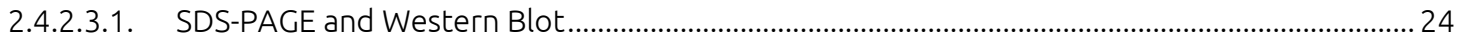

2.4.2.3.2. Immunohistochemistry and Imaging ………………………………………………………....2 24

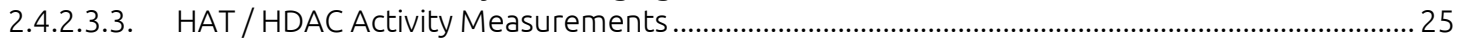

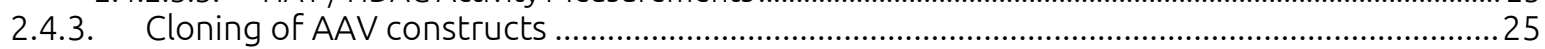

2.4.3.1. Linker-PCR from Mouse Hippocampal cDNA .................................................................................... 25

2.4.3.2. Restriction Digestion and Ligation ………………………………………………………………….... 26

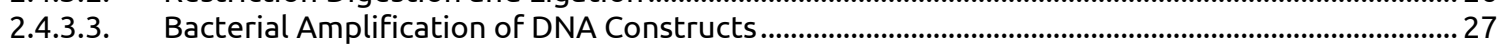

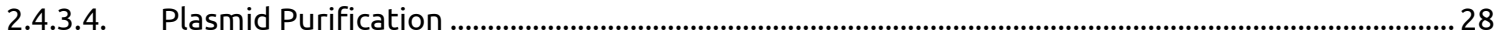

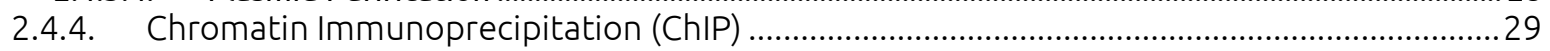

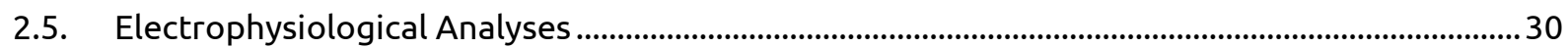

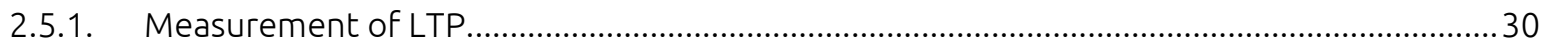

2.5.2. Measurement of Input-Output relation and Paired Pulse Ratio ..............................................30

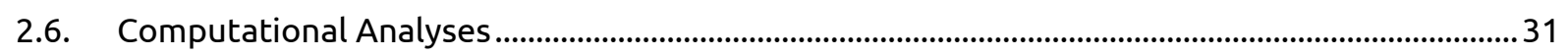

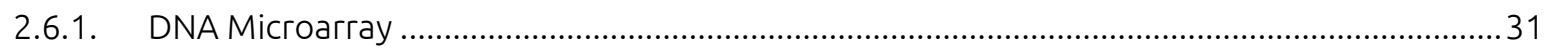

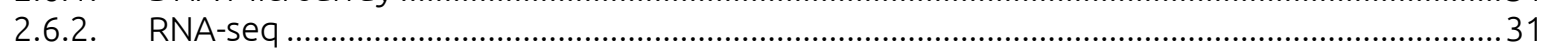

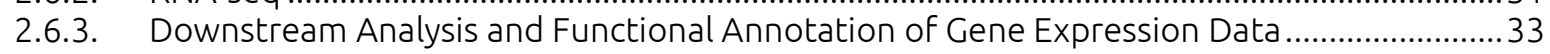

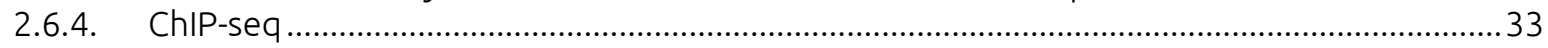

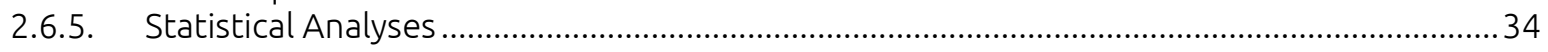

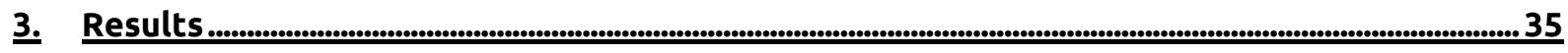

3.1. The Role of Kat2a in the Adult Murine Brain and Learning and Memory......................................36

3.1.1. Characterization of a Kat2a Tissue-Specific Conditional Knockout Mouse ...........................36

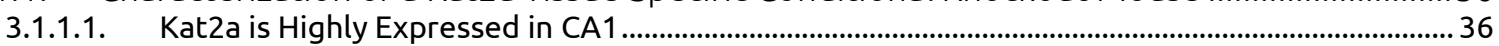

3.1.1.2. Kat2a cKO Mice Show no Overt Morphological Alterations .............................................................. 38

3.1.1.3. Kat2a cKO Mice Show Impairments in Hippocampus-Dependent LTM.............................................. 42

3.1.1.4. Kat2a cKO Mice Show Impaired Long-Term Potentiation in the CA1 ................................................ 46

3.1.1.5. Kat2a cKO Mice Show Specific Alterations in Stimulus-Dependent Gene Expression in CA1 .... 48

3.1.1.6. Kat2a cKO Mice Show Increased Levels of H4K12 Acetylation ......................................................... 54

3.1.2. Characterization of AAV-mediated Kat2a Overexpression in the Dentate Gyrus .................55

3.1.2.1. Robust AAV-mediated Expression of MYC-tagged Kat2a 14 Days after Injection ...............................55

3.1.2.2. Kat2a Overexpression Results in Spatial Memory Impairment.............................................................. 56 
3.2. Regulation of Gene Expression in Hippocampal Aging

3.2.1. Histone Acetylation in the Aged Hippocampus ......................................................................61 61

3.2.1.1. Decreased HDAC Activity is not Associated with Bulk Changes in Histone Acetylation Levels 61

3.2.1.2. Orally Administered HDACi Improves Age-Associated Memory Impairment...................................63

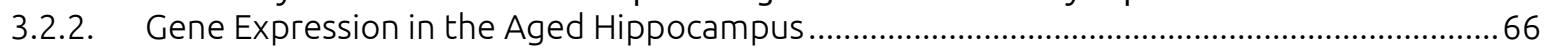

3.2.2.1. RNA Sequencing and Microarray Technology Reveal Dramatic Changes in Gene Expression ... 66

3.2.2.2. Upregulation of the Complement Component C4 Correlates with Age and is Associated with

Increased Intronic H3K9 Acetylation ................................................................................................................73

3.2.2.3. Increased C4 Protein Levels and Astrogliosis Accompany Hippocampal Aging ............................. 75

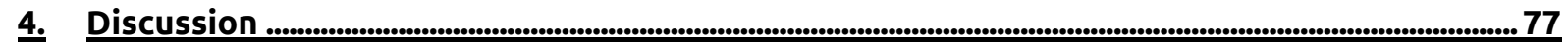

4.1. Kat2a is an Important HAT in the Hippocampus that Regulates LTM Formation

4.1.1. Kat2a conditional knockout mice exhibit several specific phenotypes related to memory

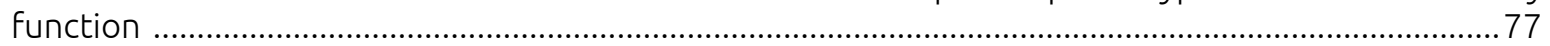

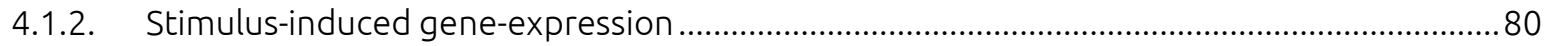

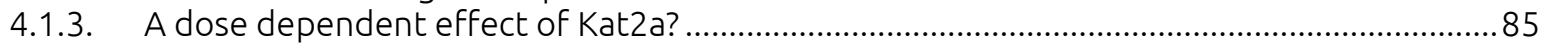

4.2. Extensive Transcriptional Changes and Decreased HDAC Activity in the Aging Hippocampus 87

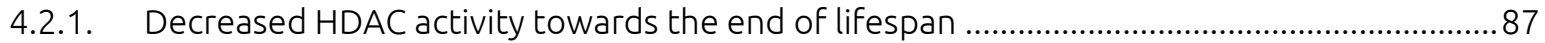

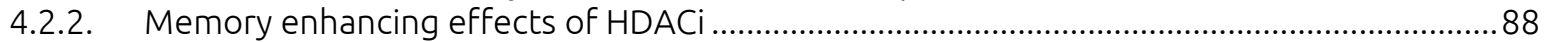

4.2.3. Transcriptional Regulation in the Hippocampus: Immune System and Chromatin ................89

4.2.4. Aging, Alzheimer's disease and the complement connection .............................................91

4.3. Closing remarks and Outlook ............................................................................................................95

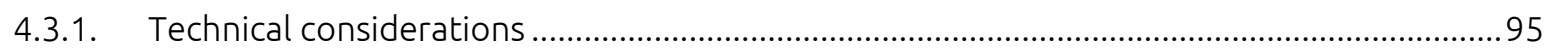

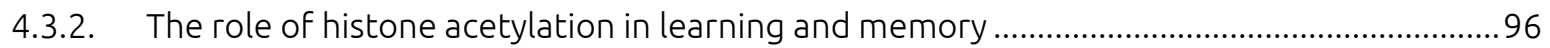

4.3.3. Implications for the synaptic tagging and capture hypothesis ............................................96

4.3.4. (Brain) Aging - an Aggregopathy? An Autoimmune Disease? Or Both!? .................................97

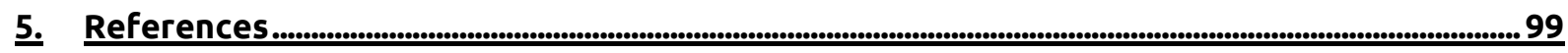

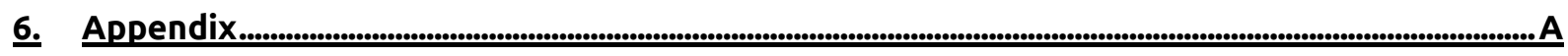

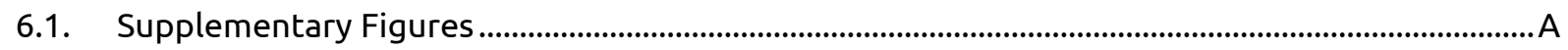

6.1.1. Expression pattern of Kat2a in the adutt murine brain from the Allen Brain Atlas................A

6.1.2. Robust AAV-mediated expression of MYC-tagged Kat2a 14 days after injection ....................A

6.1.3. Strong Accumulation of Autofluorescent Lipofuscin in Hippocampal Microglia .....................B

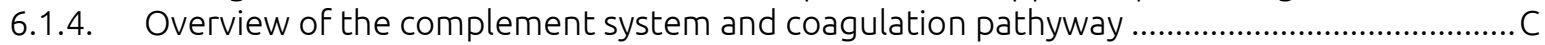

6.2. Supplementary Tables.......................................................................................................................

6.2.1. List of DEGs in the study of Kat2a function ......................................................................... D

6.2.2. Lists of significantly overrepresented GO-Terms and KEGG-Pathways in the study of Kat2a

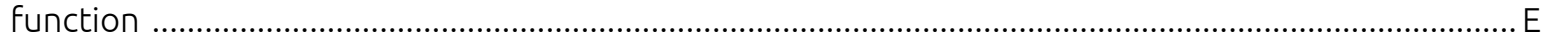

6.2.3. List of DEGs in the study of transcriptional changes during hippocampal aging ................... I

6.2.4. Lists of Significantly Overrepresented GO-Terms and KEGG-Pathways in the Study of Hippocampal Aging

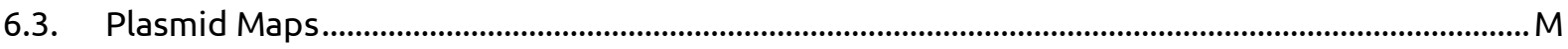

6.4. List of Primers Used in this Thesis (in order of appearance) ..........................................................

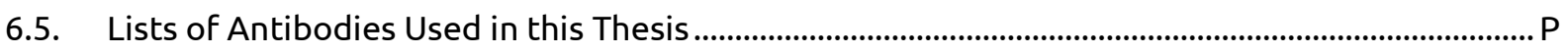

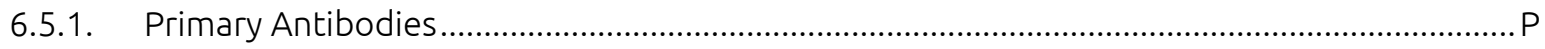

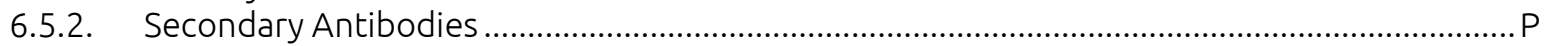

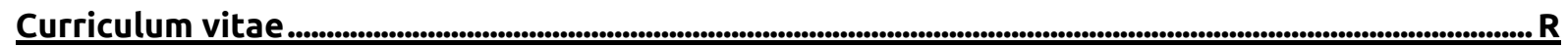




\section{Acknowledgements}

I want to express my deepest gratitude to the following people that have - directly or indirectly contributed to this thesis.

First, I want to thank my supervisor André Fischer for giving me the opportunity to carry out this thesis and also to follow up on several other, sometimes unorthodox ideas. You put a huge amount of trust, time and money in me and these projects and were always open to suggestions, discussions and always had a helping comment regardless of professional or private questions, supporting my personal and career development. I really learned a lot - not only in terms of scientific knowledge but also how to apply it. Contributing to supervision were also André Fiala and Judith Stegmüller, whom I would like to thank for supporting my work as members of my thesis committee. In addition, I want to thank Camin Dean and Gregor Eichele for being part of my extended thesis committee.

Furthermore, I want to thank my "second family for the last 4 years". All current and former have really contributed to making the lab a second home. I especially want to thank Susi (for diverse support in all circumstances and last-minute qPCRs "unter erschwerten Bedingungen"), Sanaz (for being my "Bench Buddy"), Eva (for all the scientific discussions and the last-minute microarray support), Pooja (for giving helpful comments on the thesis), Govind and Tanja (for supervising my Labrotation), and finally also Shahaf for teaching me many important lessons. Also included are my labrotation students, Ulla, Sabitha and the others, who have helped me realize some crazy ideas and taught me how to teach. Not really separated form the group mentioned above, I would like to thank the DZNE people, including Daniel (for the pleasant journey), Ulrike (for administrative support), Stefan (for honest openness, advice in countless situations, reinforcing my bioinformatics education), Vincenzo (for the marvelous job of pipelining all the data).

An integral part in the background was the ENI Team: Ali, Matthias, Sven, Frank, Heiko, Robin, Christiane and Sissi. Thank you for all the support.

To complete this thesis, several fruitful scientific collaborations were established. I'm thankful to Klaus Reymann, Raik Rönicke and Katrin Böhm (Electrophysiology @DZNE Magdeburg); Gabriela Salinas-Riester, Susanne Luthin and Lennart Opitz (TAL); Sebastian Kügler and colleagues (UMG, AAV facility). Another collarboration that is not directly implicated in this thesis but will hopefully lead to great discoveries was started with Priyanka Singh and André Fiala.

The IMPRS and GGNB deserve special attention: Michael, Sandra, the IMPRS Faculty and the GGNB team put a lot of trust in me, and I really hope I did not disappoint them.

Ich möchte natürlich auch meiner "ersten Familie" danken, meinen Eltern für all die Unterstützung und absolute Freitheit bei Studiumswahl und Entwicklung und Förderung meiner "Forschernatur" schon in frühen Jahren und meinem Bruder Sven, besonders für die zeitaufwändigen Formatierungshilfen. Besonderer Dank gilt meiner Freundin Lena, die für mich eine unerschöpfliche Quelle des Ansporns und der Liebe und Fürsorge war, und mir so geholfen hat "to get a life!".

Als ich hier in Göttingen ankam, war ich vielleicht noch eingestellt auf $M$, wie Münster, aber dann kamt ihr, Willi und Kalli, und hab mich umgestellt auf W, wie Wambo! Ohne euch wäre die Zeit in Göttingen sicherlich nicht halb so schön gewesen. Das gilt natürlich auch für die anderen Göttinger "Doppelwurstagenten". Auch für den Austausch was Wissenschaft, Karriere und Donktorandentum angeht, möchte ich mich bei euch bedanken.

Dennoch ist ein Stück Münster immer eingestellt geblieben. Ich möchte daher natürlich auch meinen Münsteraner Kommilitonen, besonders Sven, Philipp, Rüdiger, Helen, Linda, Anja und Katia, danken, für die langjährige Freundschaft über das gemeinsame Studium hinaus und die Unterstützung auf dem gemeinsamen langen Weg - von der allerersten Vorlesung bis hierher. Gleiches gilt für den Rückhalt durch meine Freunde aus der Heimat, der hoffentlich nie abreißt! 


\section{List of Abbreviations}

Please note that units described in the International System of Units (SI) as well as metric prefixes are not listed here. Similarly official gene symbols provided by the Mouse Genome Informatics resource (MGI) used in the text do not appear in this list but are rather described in the text or linked to a description on the Internet.

\begin{tabular}{|c|c|}
\hline $5-\mathrm{HT}$ & 5-hydroxytrytamine, Serotonin (-->Link) \\
\hline AAV & Adeno-associated virus (-->Link) \\
\hline AAMI & Age-associated memory impairment \\
\hline Ac-CoA & acetyl-coenzyme A (substrate for HAT-mediated acetylation) \\
\hline$A D$ & Alzheimer's disease \\
\hline AFBG & Autofluorescent background, mostly Lipofuscin (-->Link) \\
\hline a.k.a. & also known as \\
\hline ANOVA & Analysis of variance \\
\hline$A U$ & Arbitrary units \\
\hline bp & Base pairs (unit for number of nucleotides in a DNA/RNA sequence) \\
\hline CA & Ammons horn (CORNU AMMONIS) (-->Link) \\
\hline $\mathrm{CB}$ & Cerebellum \\
\hline cDNA & complementary DNA (-->Link) \\
\hline CDS & coding DNA sequence (protein-coding part, as opposed to UTR) \\
\hline $\mathrm{CFC}$ & contextual fear conditioning \\
\hline ChIP & Chromatin immunoprecipitation (-->Link) \\
\hline ChIP-seq & ChIP-DNA sequencing using next-generation technology \\
\hline cKO & conditional knockout \\
\hline DAPI & 4',6-diamidino-2-phenylindole (-->Link) \\
\hline DEG & Differentially expressed gene \\
\hline DG & Dentate gyros (GYRUS DENTATUS) (-->Link) \\
\hline DST & Differentially spliced transcript (-->Link) \\
\hline DMSO & Dimethyl sulfoxide \\
\hline DNA & Desoxyribonucleic acid \\
\hline EDTA & Ethylenediaminetetraacetic acid \\
\hline
\end{tabular}




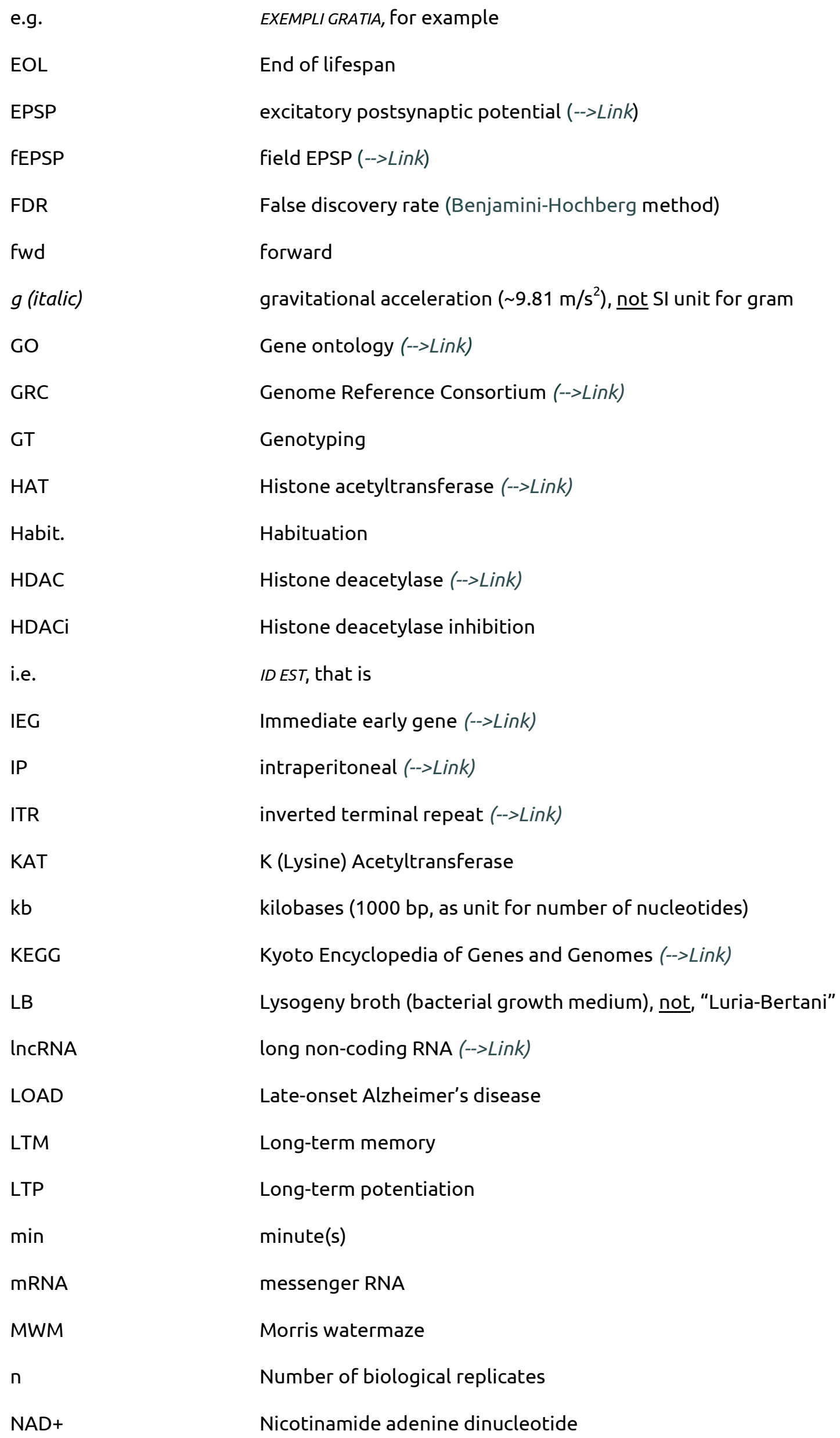




\begin{tabular}{|c|c|}
\hline NC & Neocortex (-->Link) \\
\hline NGS & Next-generation sequencing (-->Link) \\
\hline NOR & Novel object recognition \\
\hline OE & overexpression \\
\hline ON & over night \\
\hline Padj & adjusted p-value (by multiple test correction) \\
\hline PA & Polyacrylamide \\
\hline PAGE & Polyacrylamide gel electrophoresis \\
\hline PBS & Phosphate-buffered saline \\
\hline PCA & Principal component analysis (-->Link) \\
\hline PCR & Polymerase chain reaction \\
\hline $\mathrm{PE}$ & Paired-end (sequencing) (-->Link) \\
\hline Pfc & Prefrontal Cortex (-->Link) \\
\hline PLC & phospholipase C (-->Link) \\
\hline Pol2 & RNA polymerase II (-->Link) \\
\hline qRT-PCR & quantitative real-time PCR (-->Link) \\
\hline rev & reverse \\
\hline RIN & RNA integrity number (-->Link) \\
\hline rmANOVA & repeated measures ANOVA \\
\hline RNA & Ribonucleic acid \\
\hline RNA-seq & Transcriptome sequencing using next-generation technology \\
\hline RT & Room temperature \\
\hline SAHA & Suberoylanilide hydroxamic acid (HDAC inhibitor) \\
\hline SDS & Sodium dodecyl sulfate \\
\hline SE & Single-end (sequencing) (see Paired-end sequencing) \\
\hline SOP & Standard operating procedure \\
\hline sp. & SPECIES (not determined species after mention of genus) \\
\hline STC & Synaptic tagging and capture (hypothesis) (-->Link) \\
\hline STM & Short-term memory \\
\hline TAL & Transcriptome Analysis Laboratory (of the University of Göttingen) \\
\hline TBS & Tris-buffered saline \\
\hline
\end{tabular}


TQ

TR

TU

TX

UTR

vs.

$\mathrm{v} / \mathrm{v}$

$w / v$

$\times g$
Target quadrant

Training

transduction units (or infectious units) (-->Link)

Trition X 100

untranslated region (5' or $3^{\prime}$ UTR of cDNA, as opposed to CDS)

VERSUS

Volume per volume

Weight per volume

times gravitational acceleration 


\section{List of Figures}

Figure 1-1: Schematic overview of stimulus-dependent gene expression

Figure 1-2: Domain structure of the Kat2a protein. Adapted from Interpro database

Figure 2-1 The Kat2a-cKO mouse is based on the Cre-loxP system .........................................................16

Figure 3-1: Nuclear localized Kat2a is differentially expressed in adult brain regions .............................38

Figure 3-2: Anatomical alterations in Kat2 $a^{\text {ff;tg }}$ mice .................................................................................... 39

Figure 3-3: Morphological structure of the hippocampus of Kat2a cKO and control mice.....................40

Figure 3-4: Abundance and distribution of glia in the hippocampus ........................................................... 41

Figure 3-5: Behavioral analysis of Kat2a loss-of-function................................................................................. 43

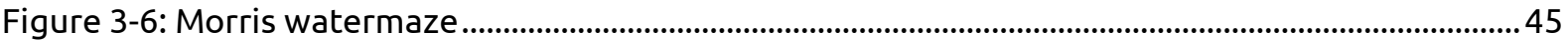

Figure 3-7: Electrophysiological analysis of long-term potentiation and paired pulse facilitation ..... 47

Figure 3-8: Schematic overview of novelty-exposure protocol for stimulus-dependent expression analysis

Figure 3-9: Stimulus-dependent gene expression determined by RNA-seq

Figure 3-10: Bulk levels of histone acetylation in the hippocampus of Kat2 $a^{f / f ; t g}$ and control mice ...54

Figure 3-11: AAV-mediated expression of Kat2a in the dentate gyrus......................................................56

Figure 3-12: Behavioral analysis of Kat2a gain-of-function 58

Figure 3-13: Morris water maze reveals impairment in spatial memory in Kat2a-overexpressing mice

Figure 3-14: EOL mice exhibit decreased nuclear HDAC activity, but no altered bulk histone acetylation.

Figure 3-15: Chronic SAHA administration in young mice transiently increases H4K12 acetylation... 63

Figure 3-16: Chronic oral SAHA administration improves long-term object recognition

Figure 3-17: Expression analysis in the aging hippocampus: Group description and quality control..67

Figure 3-18: Expression analysis in the aging hippocampus: DEGs

Figure 3-19: qRT-PCR validation and correlation with H3K9ac ..................................................................74

Figure 3-20: Increasing protein levels in the aging hippocampus ...............................................................76

Figure 4-1: The role of histone acetylation in synaptic tagging and capturing .........................................97

Figure 6-1: IN SITU hybridization image of the adult murine brain, sagittal section .....................................A

Figure 6-2: Hdac2 mRNA levels 14 days after injection................................................................................

Figure 6-3: Lipofuscin in DG and CA3 co-stained with microglia..................................................................

Figure 6-4: KEGG pathway for complement and coagulation cascades ....................................................... 


\section{List of Tables}

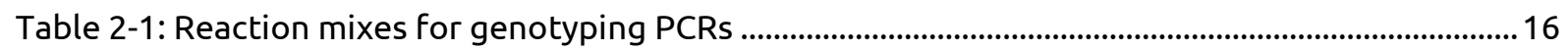

Table 2-2: Cycling conditions for both genotyping PCRs......................................................................17

Table 2-3: Reaction mixes for linker-PCRs ........................................................................................................26

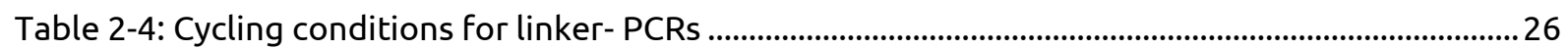

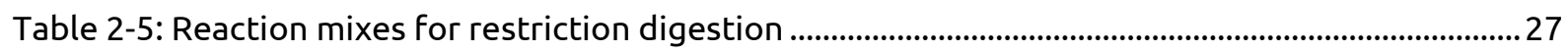

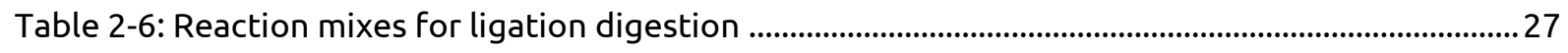

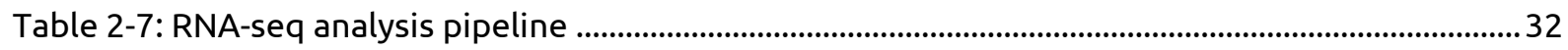

Table 2-8: Sample properties and quality control for samples used for RNA-seq in the study of Kat2a function.

Table 2-9: Sample properties and quality control for samples used for microarray and RNA-seq in the study of transcriptional changes during hippocampal aging.

Table 3-1: Color guide for the results section to help associate of figure colors with a certain

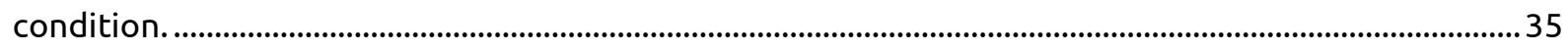

Table 3-2: mRNA levels - Tukey's multiple comparisons test after 2 way ANOVA....................................36

Table 3-3: Protein levels - Tukey's multiple comparisons text after 1 way ANOVA.....................................37

Table 3-4: Overlapping and non-overlapping DEGs .....................................................................................51

Table 3-5: Overlapping DEGs between different comparisons....................................................................69

Table 3-6: GO-Term and KEGG-Pathway analysis of merged, non-redundant lists for $3 \mathrm{~m}$ vs. $\geq 24 \mathrm{~m}$ (841 upregulated, 578 downregulated)....................................................................................................

Table 4-1: Comparison of results from genetic association studies for LOAD from the AlzGene database with differential gene expression in the aging mouse hippocampus .........................................92

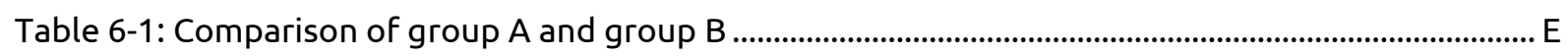

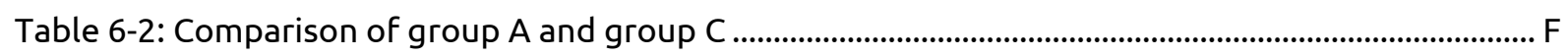

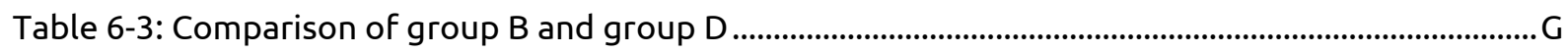

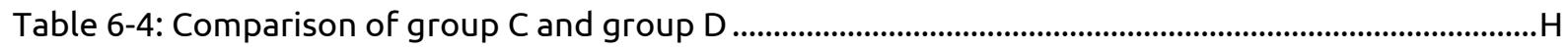

Table 6-5: Comparison of $3 \mathrm{~m}$ and $24 \mathrm{~m}$ using RNA-seq...................................................................................

Table 6-6: Comparison of $3 \mathrm{~m}$ and $\geq 28 \mathrm{~m}$ using RNA-seq ...............................................................................

Table 6-7: Comparison of $3 \mathrm{~m}$ and $\geq 28 \mathrm{~m}$ using microarrays .......................................................................

Table 6-8: List of primers used in this thesis (in order of appearance) ......................................................... 0

Table 6-9: List of primary antibodies used in this thesis (in alphabetical order) ........................................

Table 6-10: List of antibodies used in this thesis (in alphabetical order) ..................................................... 
“NAM ET IPSA SCIENTIA POTESTAS EST"

\section{"For Knowledge Itself is Power"}

"Wissen ist Macht"

SIR FRANCIS BACON

"SCIENTIA NOX EST"

\section{"Wissen ist Nacht"}

"Knowledge is Darkness"

Prof. Dr. Abdul Nachtigaller 


\section{Preface}

Dear reader,

Scientific research is a fast-paced business with new results, reviews and opinions of thousands of researchers worldwide published every day. Digital, accelerated publishing and a steadily growing number of scientific journals make it increasingly difficult to navigate through the jungle of literature. While the author of this dissertation is a supporter of open access digital publishing, he feels that for the individual, increasing complexity can only be faced using digital armaments like reference management software and data-and-text mining tools. Already using the former, the latter however still seems to be a special discipline of computer scientists and not widely usable.

This manuscript was carefully crafted and established as well as recent literature was cautiously selected for citation. However, for the reasons mentioned above, not all publications that might refer to the topics presented in this dissertation could be discussed or cited. Moreover, new papers have been appearing during the process of writing that did not find their way into the final manuscript. New publications are only recognized until the date of 01.03.2013. The author apologizes for any inconvenience caused by these circumstances.

Although you may read this document in its printed form, indeed the manuscript was written and edited on a computer. The text is enriched with hyperlinks to navigate within the document (dark blue font color) and to access additional information on the Internet (dark green font color). To experience the full potential of informational enrichment and easier access to information relevant to you, the author highly recommends reading the digital version of this document.

The reader may notice, that reagents and devices i2n the "Materials and Methods" section are also linked. In addition, the supplier is given in brackets. However, the author consciously desisted from denoting the city or country a supplier may be based in, since in the globalized and fast changing life sciences market, where companies, fuse, split and outsource within increasingly short periods, the model of a centralized headquarter is simply outdated and does not provide any additional informational use to the reader. On the contrary, readers interested in making use of the experimental procedures presented here shall make use of the meta-information that is represented in linking materials and devices with their official description on the world wide web.

Please also note that gene names for mouse genes used in this thesis are official gene symbols provided by the Mouse Genome Informatics resource (MGI) based on genome assembly GRCm38. Human gene names are official gene symbols provided by the HUGO Gene Nomenclature Committee (HGNC) based on genome assembly GRCh37. Furthermore, it should be recognized that words in a language other than English (American English is used throughout the thesis) are denoted in ITALIC SMALL CAPITALS. 


\section{Summary}

Evolution has equipped multicellular organisms with ever more sophisticated means of processing information about the environment they live in. The hippocampus has been shown to be one of the most important structures of the mammalian brain for information processing with respect to learning and memory formation.

In general, memories stored for seconds to minutes are known as short-term memories (STMs), while long-term memories (LTMs) may be stored for hours, days and up to years. Research on the cellular and molecular mechanisms of memory consolidation has revealed that LTM formation as well as a cellular correlate of this process - late-phase long-term potentiation (LTP) - depend on DE Novo protein synthesis and transcription. The synaptic tagging and capture (STC) hypothesis has been formulated as a theoretical basis of this process.

Chromatin plasticity, including dynamic histone acetylation has been demonstrated to play a positive role in long-term memory consolidation and regulation of plasticity-related transcription. Inhibition of histone deacetylases (HDACi) has beneficial effects in several disease models and enhances memory formation across multiple species. Loss of histone acetyltransferase (HAT) function on the other hand has negative effects on memory consolidation. Kat2a is a HAT associated with stimulus-dependent transcriptional activation. However, its function and targets in the adult brain have not been explored yet.

With increasing human life span, aging is becoming a major challenge in modern societies. As in many other aspects, the brain also holds an exceptional position when it comes to aging. One of the earliest symptoms of the aging brain is age-associated memory impairment (AAMI), which is a normal though commonly undesirable process, manifesting itself by difficulties in acquisition of new memories and by increased forgetfulness. Brain aging is also accompanied by massive transcriptional changes. However, a detailed, homogenous picture of the transcriptome of the aging mouse hippocampus, especially towards the end of an individual's life span, has not been drawn yet. Accumulating evidence suggests that chromatin-related mechanisms may be involved in the regulation of transcriptional aging. However, data on the contribution of histone acetylation remains incomplete.

Here, we provide extensive evidence supporting a role for Kat2a in learning and memory. The data shows strong Kat2a expression in the hippocampal neurons, especially in the CA1. Using elaborate behavioral testing, we show impairment of hippocampus-dependent LTM formation upon Kat2a deletion from excitatory forebrain neurons. This finding is further supported by electrophysiological data revealing impaired LTP in the CA1 region. Interestingly, stimulus-dependent mRNAome profiling in the CA1 of conditional knockout and control mice following a novelty-exposure paradigm showed downregulation of several genes related to neuronal activity, which are likely target genes of Kat2a activity. Interestingly, Kat2a overexpression in the DG did not result in enhancement of LTM formation, and even led to impaired performance in the Morris water maze, 
used for spatial memory testing. The findings are discussed with respect to stimulus-dependent gene expression during memory consolidation and in the light of the STC hypothesis.

In a second set of experiments we assessed histone acetylation in the hippocampus of aged mice. While we found no evidence for altered levels of bulk histone acetylation or differential HAT activity, we observed reduced HDAC activity in mice towards the end of lifespan (EOL), that was not associated with reduced expression of $\mathrm{Hdac2}$ or Hdac3. Assuming this to be a compensatory mechanism, we tried to facilitate this compensation at an earlier stage using HDACi. Indeed, longterm HDACi treatment rescued the AAMI phenotype that is observed in aged mice.

In addition, transcriptional changes that accompany the aging process in the hippocampus were detected using whole-transcriptome mRNA sequencing as well as microarray technology. Together, both methods revealed a transcriptional signature of aging highly associated with a proinflammatory milieu, which may be caused by ineffective aggregate clearance. Increased intragenic $\mathrm{H} 3 \mathrm{~K} 9$ acetylation was associated with at least one of these genes, $C 4 b$, demonstrating activatory regulation. Implications of these findings for brain aging in general as well as late-onset Alzheimer's disease in particular are presented and discussed. 


\section{Introduction}

\subsection{Learning and Memory}

\subsubsection{Memory Systems}

Evolution has equipped multicellular organisms with ever more sophisticated means of processing information about the environment they live in. In the past century scientists have identified two pivotal, yet very dissimilar mechanisms of environmental information processing and adaptation: Perception and genome-environment interaction (referred to as epigenetics; Holliday, 2006; Waddington, 1953). Though these processes seem to be unrelated at the first glance, they share an integral similarity, since they provide an internal representation of the environment. The first mechanism relies on sensory processing of diverse external stimuli and is represented in a complex network of cell-to-cell communication. With the development of a brain, multicellular organisms have evolved a unique organ for the first task, made up of a cell type that has brought cell-to-cell communication to perfection. The second mechanism represents information in the form of a transcriptional profile that adapts to extracellular signals to maintain homeostasis (see 1.3.2). Even if this information is represented on very different levels of abstraction, this information can be preserved beyond the actual stimulus. We call information stored this way memory.

The term memory (from Latin MEMORIA) in general refers to storage and retrieval of information. Thus, the term can be used in many different ways with diverse connotations depending on the context or scientific discipline, ranging material science (shape memory, smart metal) over computer science (data storage) and cell biology (transcriptional memory) to neuroscience and psychology (engram) and even sociology (culture, collective memory). In this thesis, the term memory will be exclusively referring to the mechanisms of storing multimodal sensory information in the brain, which will lead to so-called engrams or memory traces (Semon, 1921). Learning will be defined as the process of generating this engram, also referred to as encoding. Though it is still unclear what the physical correlate of the engram is, several mechanisms have been proposed to explain how memory traces can manifest themselves in the physical world of neurophysiology (Bruce, 2001; Garner et al., 2012; Liu et al., 2012; Reijmers et al., 2007). However, none of these theories is able to explain all aspects of memory (Sakaguchi and Hayashi, 2012).

A major challenge in finding the nature of the engram is the variety of forms of memory and it seems plausible that different types of information are stored in different ways. While these distinct types of memories can be observed by introspection and intuition, systematic research has classified them into two major subtypes called declarative and procedural memory. The declarative or explicit memory system can be further divided into semantic memory (facts) and episodic memory (autobiographic events), which both require attention (Eichenbaum, 1997; Tulving and Donaldson, 1972; Ullman, 2001). The procedural or implicit memory system is used to describe skill acquisition or other forms of learning, which do not rely conscious awareness (Fitts, 1954; Ullman, 2001). Both subtypes of memory rely on different though partly overlapping brain regions (Gazzaniga, 2004). 


\subsubsection{The Hippocampus as an Anatomical Memory Hub}

The hippocampus has been shown to be one of the most important structures of the brain related to learning and memory formation. The first indications of its relevance came from human explantation experiments, where parts of the medial temporal lobe including the hippocampal formation were removed, which resulted in severe anterograde and retrograde amnesia in the patients (Corkin, 2002; Lah and Miller, 2008; Scoville and Millner, 1957). Further support came from animal studies, where the hippocampus was physically or pharmacologically lesioned (Barker and Warburton, 2011; Iordanova et al., 2009; Kim and Fanselow, 1992). Compared to rodents, humans have a small hippocampus in relation to total brain volume, though the subregional anatomical structure has largely been preserved during evolution (West, 1990). Interestingly, hippocampal volume has also been correlated to spatial memory capacity, between species as well as among individual humans (Jacobs et al., 1990; Maguire et al., 2006; Woollett and Maguire, 2011).

To perform the task of storing information about the outside world or associations between external and internal stimuli, the hippocampus needs to be well connected to other brain regions and to integrate sensory information. One of the main excitatory hippocampal afferents is the perforant pathway that connects the entorhinal cortex with the Dentate Gyrus (DG) subregion of the hippocampus. The entorhinal cortex in turn receives pre-processed input from brain areas of multiple sensory modalities. In addition, the entorhinal cortex is also the major site receiving hippocampal output via the subiculum. It then relays the information to all neocortical areas in the brain. The entorhinal cortex can therefore be viewed as the major interface and information integrator between the hippocampal formation and the neocortex (Buzsáki, 1996; Lavenex and Amaral, 2000). Inside the hippocampus the main excitatory circuit involves the DG to CA3 connections (Mossy fibers), CA3 to CA1 connections (Schaffer collaterals) and CA1 to Subiculum connections. The CA1 has additional efference to the prefrontal cortex (PfC) and to the entorhinal cortex directly (Andersen et al., 2007).

In addition, the hippocampus is subject to several modulatory inputs from other neurotransmitter systems, including dopaminergic input (mainly originating in the SUBSTANTIA NIGRA) or serotonergic input (mainly originating in RAPHE NUCLEI). Hippocampal neurons express several of the various receptors to respond to these neurotransmitters. For example, the mouse genome encodes 16 known serotonin (5-HT) receptors, classified in 7 families. In the CA1 region 10 of these have been found, with differential expression in pyramidal neurons and inhibitory interneurons (Andrade, 1998). Each of these mostly G-protein-coupled receptors has a different modulatory effect on electrophysiological properties of neurons. Thus, serotonergic modulation of hippocampal function is rather complex and critically depends on the set of receptors expressed in a certain region or cell (Andrade, 1998; Seifert, 2010).

Though any of the three subfields of the hippocampus are necessary for memory formation, they are morphologically distinct and play dissociable roles. While the DG with its granule cell neurons is one of the two sites in the brain where adult neurogenesis occurs, the CA regions harbor pyramidal neurons and are involved in multiple sudivisions that form individual ensembles and microcircuits to 
account for different types of information (Moser and Moser, 1998). This goes along with dissimilar functions for different forms of learning and the multiple types of memory (Moser and Moser, 1998). For example, CA3 and CA1 are both involved in fear extinction learning, while only CA1 is necessary for is required for retrieval of the learned fear (Ji and Maren, 2008).

\subsubsection{Long-Term Memory Consolidation}

Another way to distinguish different forms of memory is the time that elapses between encoding and retrieval. In general, memories stored for seconds to minutes are known as short-term memories (STMs). STM is overlapping, but not identical, with the concept of working memory, in which the memory trace can be subject of active, "online" manipulation (Baddeley, 2003). Long-term memories (LTMs) on the other hand, may be stored for hours, days and up to years. The process of transformation of an STM trace toward a stable LTM is known as memory consolidation. This term was first coined more than a hundred years a ago (Müller and Pilzecker, 1900) and is still used to describe that the formation and stabilization of LTMs is a time-dependent process rather than an instantaneous event or shift (Dudai, 2004; Lechner et al., 1999; McGaugh, 2000). Memory consolidation happens in two steps, of which the first, termed synaptic consolidation, is accomplished within minutes to hours after memory acquisition. The second step, called systems consolidation, involves the transfer of hippocampus-dependent memories to cortical areas and completed over a considerably larger time scale. More recently, a third step has been proposed, reconsolidation, in which a stable memory becomes labile and can be modified again. The existence of this subsequent step, however, is still under debate (Dudai, 2004; Dudai and Eisenberg, 2004).

\subsubsection{Protein Synthesis and Transcription}

Research on the cellular and molecular mechanisms of memory consolidation has revealed that memory can also be classified by whether it is dependent on DE NOVO protein synthesis or not. Early studies mainly focused on protein synthesis inhibitors to establish a causal connection between translation and memory consolidation (Davis and Squire, 1984; Flexner et al., 1963; Schwartz et al., 1971). Local protein synthesis by translation of dendrite-localized mRNA at the post-synapse is one of the proposed mechanisms (Bramham and Wells, 2007; Schuman et al., 2006).

Since, however, translation is only the second step in gene expression and molecular signaling cascades also travel to the nucleus, it soon became evident that transcription is also a necessary step towards creating a stable memory trace. This connection has mainly been established by inhibiting the RNA polymerase II (Pol2) (Castellucci et al., 1986; Igaz et al., 2002, 2004; Montarolo et al., 1986; Da Silva et al., 2008).

\subsubsection{Long-Term Potentiation and the Synaptic Tagging and Capture Hypothesis}

Long-term potentiation (LTP) is believed to comprise a neurophysiological correlate of a memory trace. This phenomenon occurs in a given neuron or population in response to a strong synaptic stimulation, e.g. a high frequency depolarization, and results in an elevated excitatory post-synaptic potential (EPSP) to subsequent low frequency inputs (Bliss and Gardner-Medwin, 1973; Bliss and Lømo, 1973; Lømo, 2003). This establishes a "synaptic memory" that can last up to several hours in 
vitro and even days in the living animal (Bliss and Gardner-Medwin, 1973; Bliss and Lømo, 1973; Douglas and Goddard, 1975). Furthermore, the mechanism serves as a coincidence detector, since high-frequency input may also originate from multiple independent neurons or synapses, which happen to reach a given neuron at the same time (i.e. within a short interval) by coincidence.

Interestingly, LTP also resembles the different phases of memory consolidation, as LTP can as well be subdivided into several phases. Like the different phases of LTM consolidation, the phases of LTP range from early phases that are independent of protein-synthesis to later phases that do depend on protein synthesis and transcription (Nguyen et al., 1994; Reymann and Frey, 2007).

However, synaptic inputs are highly localized while the supply of newly synthesized proteins to mediate plastic effects of these inputs from the soma is ignorant to where the signaling originated. How should the new proteins of neuron "know" where they are needed to establish a more effective connection to the high-frequency-firing neighbor? To elucidate underlying mechanisms, the synaptic tagging and capture (STC) hypothesis has been formulated (Frey and Morris, 1997). Upon stimulation a synaptic tag is set at the stimulated synapse. Depending on the strength of the stimulus nuclear signaling will lead to production of new plasticity-related proteins that recognize the tag and will thereby localize preferentially to synapses that underwent stimulation. Again, coincidence of weak stimulation at one synapse and strong stimulation at another may lead to capturing of the tag set at the weakly stimulated synapse, thus resulting in late LTP at both synapses (Martin et al., 2000; Reymann and Frey, 2007).

In addition, the hypothesis may also connect protein synthesis-dependent and transcriptiondependent mechanisms of LTP and LTM: While tag setting and even spatially confined capturing may be achieved by local protein translation, capturing by soma-supplied, newly generated transcripts and proteins is dependent on nuclear signaling.

As yet the STC hypothesis is a theoretical concept derived from experimental evidence IN VITRO and, most recently, also IN VIVO (Shires et al., 2012), but though there are some promising candidates, the exact nature of the tag and the precise molecular mechanisms of the two steps are still elusive. Also, the question of whether dendritic translation is sufficient to explain the localization problem mentioned above remains, since it has recently been shown that the abundance of mRNA at dendrites had been underestimated (Cajigas et al., 2012). Interestingly, however, the STC concept has recently been applied at a different level of complexity and is used to describe observations called behavioral tagging (Moncada and Viola, 2007). In this intriguing phenomenon, a behavioral learning protocol that would not suffice to induce a stable long-term memory trace is paired with an unrelated behavioral stimulus, e.g. novelty exposure, which together will result in a consolidated long-term memory (Ballarini et al., 2009; Barco et al., 2008; de Carvalho Myskiw et al., 2013; Moncada and Viola, 2007; Moncada et al., 2011). 


\subsubsection{The mouse as a model organism for the study of learning and memory}

Today, models for mammalian hippocampal function in learning and memory are mainly rats and mice. The latter are preferentially used for the study of genetic and molecular mechanisms underlying memory and to model individual aspects of human disease, since mice can easily be genetically modified and have a short generation time. Researchers have elaborated developed tests to assess the content of a specific memory in the laboratory by observation of animal behavior. Different tests are used for different types of memory. In the following, I describe two of the most common memory tests used for mice.

Classical pavlovian fear conditioning is one of the most commonly used tests for associative memory (Blanchard and Blanchard, 1969; Fendt and Fanselow, 1999). In this paradigm mice are trained to associate a novel environment (conditioned stimulus) with an electric foot shock (unconditioned stimulus). The readout for formation of an associative memory is an inborn behavioral response to fear, which is expressed by freezing. The percentage of the time the animal displays freezing behavior during context reexposure correlates with memory strength. This type of contextual fear conditioning (cFC) has been shown to be hippocampus-dependent ( $\mathrm{I}$ and Maren, 2008; Kim and Fanselow, 1992).

Another hippocampus-dependent memory test is the so-called Morris Water Maze (MWM), invented by Richard Morris in 1984 (Morris, 1984). This test is used to investigate spatial memory in rodents. Mice are placed in a pool filled with opacified water and need to find a platform that is submerged just under the water surface to escape the maze. The pool is surrounded by visual cues mice can use as landmarks to orient themselves and to locate the hidden platform. During the initial trials, mice have to learn that the platform is the only way out, and since they do not see the platform, they have to be guided to it by the experimenter. With repeated training mice generally show decreasing escape latencies, indicating increasing awareness of the task procedure and better orientation in the maze. Finally, the platform is removed and mice are allowed to swim in the pool for a certain time. During this time the time spent in each of four virtual quadrants of the pool is measured. The target quadrant (TQ) is defined as the quadrant where the platform was originally placed. The fraction of time spent in this TQ can be used to measure memory strength (Bromley-Brits et al., 2011; Morris, 1984).

In addition, several behavioral tests have been developed to assess other aspects of behavior like exploratory behavior, curiosity and anxiety. Yet, the read out of memory-testing tasks may depend on these parameters and may lead to false results if not accounted for. 


\subsection{Brain Aging}

The aging process affects the whole body and does not spare the brain. As in many other aspects, the brain also holds an exceptional position when it comes to aging. This is largely due to the fact that neurons are terminally differentiated, post-mitotic cells that cannot be replenished after events of cell loss, though there are reports suggesting that adult neurogenesis, confined to the DG and the subventricular zone, could play a role to ameliorate at least some of the age-associated decline (Deng et al., 2010; Praag et al., 2005)

Improved medical care and sanitation standards together with enhanced access to sources of nutrition have resulted in progressively increasing human life span in the past century, resulting in huge benefits as well as posing a challenge to modern society. At the moment it is not clear when this trend will slow down and what the biological or medical limit to aging is. Research on the physiological conditions at advanced ages and towards the end of an organism's life span is therefore invaluable for the future of our societies. Here we use aged mice as a model for mammalian brain aging. Though mice are warm-blooded mammals and therefore share certain similarities with humans and though we further assume that processes towards the end of an organism's life span are comparable, a simple conversion of ages (e.g. 2 "mouse years" correspond to 70 "human years") is inadequate, since mice exhibit quite distance metabolic features due to their much larger surface-to-volume ratio.

\subsubsection{Age-Associated Memory Impairment}

One of the earliest expressions of the aging brain is age-associated memory impairment (AAMI). This is a normal though undesirable process, often manifesting itself by difficulty in the acquisition of new memories and increased forgetfulness along with decreased working memory capacity. Notably, it is very difficult to distinguish between normal and pathological cognitive decline, since mild cognitive impairment can be a first sign of dementia or neurodegenerative diseases like Alzheimer's disease (Hedden and Gabrieli, 2004).

As the hippocampus is the main hub for memory formation and retrieval research on AAMI has invested a great deal of resources in the study of changes within this brain structure. This includes neurophysiological analyses as well as studying cellular and transcriptional changes.

\subsubsection{Transcriptional Changes in Aging}

Interestingly, distinct brain regions do not age at the same rate (Moroz and Kohn, 2010; Raz and Rodrigue, 2006; Xu et al., 2007). Several studies have investigated the transcriptional profiles of different brain regions in different species and came to heterogeneous results (Bishop et al., 2010; Blalock et al., 2003, 2010; Finch and Morgan, 1990; Loerch et al., 2008; Lu et al., 2004; Pawlowski et al., 2009; Pletcher et al., 2002; Verbitsky et al., 2004; Xu et al., 2007; Yankner et al., 2008; Zahn et al., 2007). Also within the same structure, e.g. the hippocampus, and the same or closely related species, results are not strikingly consistent. This likely has diverse reasons associated with 
experimental procedures, including husbandry conditions, the actual age of the animals used, sex differences, differences in methodology and finally also limited information on the mouse genome and transcriptome.

One of the most significant efforts to determine the transcriptional changes associated with aging in the mouse is the AGEMAP project (Zahn et al., 2007), which includes the data by Xu ET AL. (Xu et al., 2007). Between mice of 1, 6, 16 and 24 months of age, a total of almost 9000 genes haven been found to change in 16 tissues (Zahn et al., 2007). However, for the hippocampus there were only 2 genes significantly correlated with increasing age. Other studies tried to find similarities across mammalian species, including humans, and found only a small subset of gene expression changes to be conserved (Loerch et al., 2008).

\subsubsection{Chromatin-Related Changes in Aging}

Several authors have pointed out that chromatin-related ("epigenetic") mechanisms have emerged as important modulators of the aging process in the brain (Kosik et al., 2012; O'Sullivan and Karlseder, 2012; O'Sullivan et al., 2010; Penner et al., 2010a, 2010b; Stilling and Fischer, 2011). This general agreement is based on various observations that are associated with aging and alter chromatin function, including increased DNA damage by reactive oxygen species (ROS), shortened telomeres and loss of core histones (O'Sullivan and Karlseder, 2012). Recent findings from the host laboratory support the notion of loss of chromatin plasticity in response to external stimuli (Peleg et al., 2010). Further evidence for participation of chromatin regulators was derived from investigation of the sirtuin (SIRT) family of histone deacetylases. Overexpression of SIRTs generally has been shown to have beneficial effects on genomic stability during aging and overall life span in several organisms (Kanfi et al., 2012; Michan and Sinclair, 2007; Oberdoerffer et al., 2008). This effect is like attributed to the close interaction of SIRTs with cellular metabolism, due to their localization ${ }^{1}$ and their use of nicotinamide dinucleotide (NAD+) as a cofactor (Braidy et al., 2011).

\footnotetext{
${ }^{1}$ SIRTs 3,4 and 5 are localized to mitochondria
} 


\subsection{Chromatin Plasticity and Regulation of Transcription}

Recently, a deeper connection between the two mechanisms of environmental information processing, perception and epigenetics, has been established, sometimes referred to as neuroepigenetics. This new discipline tries to unravel the dynamic plastic changes in transcriptional regulation in neurons upon stimulation or in disease.

Neuronal networks are plastic in terms of connectivity and communication strength. In turn, all cellular components of an individual neuron, including the nucleus, need to be plastic, so that they can undergo physical, chemical or morphological changes of structure or function in order to respond to external stimuli. Chromosomal DNA in the nucleus is tightly packed and highly decorated with DNA-binding proteins and RNA molecules, which together make up the nuclear chromatin. It is therefore not surprising that also nuclear architecture and chromatin are subject to plastic changes, which is necessary for highly orchestrated and dynamic alterations in the production of new proteins and regulatory RNAs. Chromatin is an essential integrator of cellular signaling cascades and a crucial regulator of transcriptional activation or shutdown. To execute this function, chromatin can undergo diverse chemical modifications that act as signals for the transcriptional machinery. These include DNA modifications like methylation and hydroxymethylation but also covalent modification of histone proteins, which make up the nucleosomes that are wrapped by DNA in a "beads-on-astring" configuration. In particular, the $\mathrm{N}$-terminal histone tail domains that project from of the nucleosome are subject to a plethora of covalent modifications, including methylation, acetylation, phosphorylation and ubiquitination, to name just a few. These modifications have different effects on intra- and inter-nucleosome association and nucleosome-DNA binding affinity and also serve as binding sites for other regulatory proteins and the transcriptional machinery.

\subsubsection{Histone Acetylation}

The addition of an acetyl group to lysine residues of histone tails has been one of the first demonstrated histone modifications (Murray, 1961) and its activatory role in transcription was discovered early (Allfrey et al., 1964; Dion et al., 2005; Durrin et al., 1991). Acetylation is thought to influence transcriptional activation by two non-mutually-exclusive mechanisms. First, addition of an acetyl group removes the positive charge from the protonated lysine residue and thereby reduces the electrostatic interaction with the negatively charged DNA. In turn, the tight binding of DNA and nucleosome loosens and the DNA is more easily accessible to the transcriptional machinery. Second, acetylated lysine residues function as binding sites themselves and recruit protein partners to stimulate transcription, a function that is mainly carried out by proteins harboring a bromodomain, which is a protein domain that specifically binds to acetylated lysine residues (Ai et al., 2011; Arrowsmith et al., 2012; Hargreaves et al., 2009; Hassan et al., 2007; Kanno et al., 2004; LeRoy et al., 2008; Winston and Allis, 1999).

Histone acetylation itself is regulated by the counteracting enzymatic activity of histone acetyltransferases (HATs) and histone deacetylases (HDACs). Since histone acetylation is generally associated with activation of transcription, HATs are suggested to stimulate transcription, while 
HDACs are supposed to inhibit active transcription (Carrozza et al., 2003; Ekwall, 2005; Kouzarides, 2007; Roth et al., 2001). In terms of genomic location, the activatory function of histone acetylation is mostly associated with the promoter region. However with the advent of large-scale genomewide occupation studies using chromatin-immunoprecipitation (ChIP) followed by massive parallel sequencing, histone acetylation has also been shown to associate with other genomic regions, including the coding region of actively transcribed genes, and also telomeres (Chepelev et al., 2012; Durrin et al., 1991; Ginsburg et al., 2009; Hargreaves et al., 2009; Johnsson and Wright, 2010; Peleg et al., 2010; Värv et al., 2010; Zhou et al., 2011a). The exact function of a given modification however, remains one of the main mysteries in the field.

\subsubsection{Histone acetylation in learning and memory}

After the discovery that certain forms of memory depend on protein and RNA synthesis, it was only a matter of time until the first groups showed involvement of several of the above mentioned chromatin modifications in memory consolidation: Histone acetylation in the CA1 region of the hippocampus was again the first modification shown to correlate with memory formation (Levenson et al., 2004; Swank and Sweatt, 2001) followed by histone phosphorylation (Chwang et al., 2006), DNA methylation (Miller and Sweatt, 2007; Miller et al., 2008) and histone methylation (Grinkevich, 2012; Gupta et al., 2010; Kerimoglu et al., 2013). To date, histone acetylation has been found associated with learning and memory in diverse settings and across a wide range of species, including DROSOPHILA MELANOGASTER (Fitzsimons and Scott, 2011), APIS MELLIFERA (Merschbaecher et al., 2012), HELIX SP. (Danilova and Grinkevich, 2012), CHASMAGNATHUS SP. (Federman et al., 2009) rodents and primates (Gräff and Tsai, 2013a). In general, increased histone acetylation is associated with facilitation of memory consolidation (for recent reviews see Gräff and Tsai, 2013a, 2013b). The proposed mechanism of this facilitation is based on the positive effect of histone acetylation on transcription, and in the context of learning and memory especially on those genes associated with synaptic plasticity (McQuown and Wood, 2011).

\subsubsection{Inhibition of Histone Deacetylase Activity}

Inhibitors of HDAC activity are used to transiently increase histone acetylation. This has potential advantages, such as to facilitate the induction of certain genes that are required in a given situation, e.g. in response to an external stimulus. This is the reason, why the same HDAC inhibitor can have different effects deepening on the cell type it is applied.

To date several small-molecule HDAC inhibitors have been identified or specifically designed (Fischer, 2010). Interestingly, also several naturally occurring substances also exhibit HDACi activity. These include sulphoraphan and related isothiocyanates (abundant in broccoli), lactate and Kaempferol (Berger et al., 2012; Dashwood and Ho, 2008; Latham et al., 2012). However, most of the HDAC inhibitors available today are rather unselective towards the different HDAC isoforms (Fischer, 2010). Since these HDAC isoforms have only partially overlapping functions it is desirable to amend the HDACi portfolio with additional, more specific molecules.

Suberoylanilide hydroxamic acid (SAHA), also known as Vorinostat, is a small-molecule HDAC inhibitor that has been shown to be able to cross the blood brain barrier and is already clinically 
applied for oral treatment of advanced refractory cutaneous T-cell lymphoma under the trade name Zolinza® (Mann et al., 2007; Stowell et al., 1995).

\subsubsection{Stimulus-Dependent Regulation of Gene Expression}

Cellular life is a constant struggle against the laws of thermodynamics. Entropy tends to increase and chemical reactions tend to reach an equilibrium stage. Yet, cells decrease their internal entropy and maintain a chemical milieu away from equilibrium for enzymes to carry out their functions, as Erwin Schrödinger already pointed out in his famous book "What is life?" in 1944 (Schrödinger, 1992). In that sense equilibrium is equal to death. The process of maintenance of this cellular interpretation of stability is called homeostasis, a term first coined by Walter Cannon in 1929 (Cannon, 1929). Homeostasis in turn requires a sort of "housekeeping", i.e. a set of tasks that is necessary for survival and structural and functional maintenance. These tasks are mainly carried out by proteins encoded by so-called "housekeeping genes", which are very robust against intra- or extracellular disturbances (Butte et al., 2001).

Though their interior may be fairly homeostatic, the extracellular environment is likely to change over time. Thus cells have to respond to changes in environment in order to maintain their internal state and have evolved a plethora of chemical and physical receptors and signaling pathways for exactly this purpose. These mechanisms usually result in plastic changes of the interior organization of a cell, including changes in transcriptional activity. Thus, transcription that is activated or deactivated by such a signal can be called stimulus-dependent transcription. In that sense, all changes in gene expression could be called stimulus-dependent.

In a multicellular organism, the proximate environment is very likely to consist of other cells, so that receptors and signaling mechanisms may be used for cell-to-cell communication. In this respect neurons are perhaps the most remarkable cell types, since they have evolved to form complex cellular networks and offer multiple channels for communication. Here, the term stimulus is defined as an external signal that leads to altered neuronal activity and in turn to plastic changes in the activated neurons.

Interestingly, a class of genes that are upregulated upon stimulation is shared by various cell types. These genes are transcribed almost immediately after the signal has reached the - in many cases already pre-stalled, yet paused - transcriptional machinery, which has earned them the name "immediate early genes" (IEGs), a.k.a. primary response genes (Hargreaves et al., 2009; Morgan and Curran, 1989). One of the most well studied immediate early genes is the Fos gene (a.k.a. cFOS), which is, like many other IEGs, a transcription factor itself (Morgan and Curran, 1988). Thus, once expressed, many IEGs stimulate further transcription of their respective target genes, which provides a way of amplifying a signal while diverting it at the same time. The targeted genes in turn are the genes that are needed for a specific task, here referred to as effector genes (Figure 1-1). In neurons these may comprise plasticity-related genes like additional ion channels and structural components of the post-synapse. 


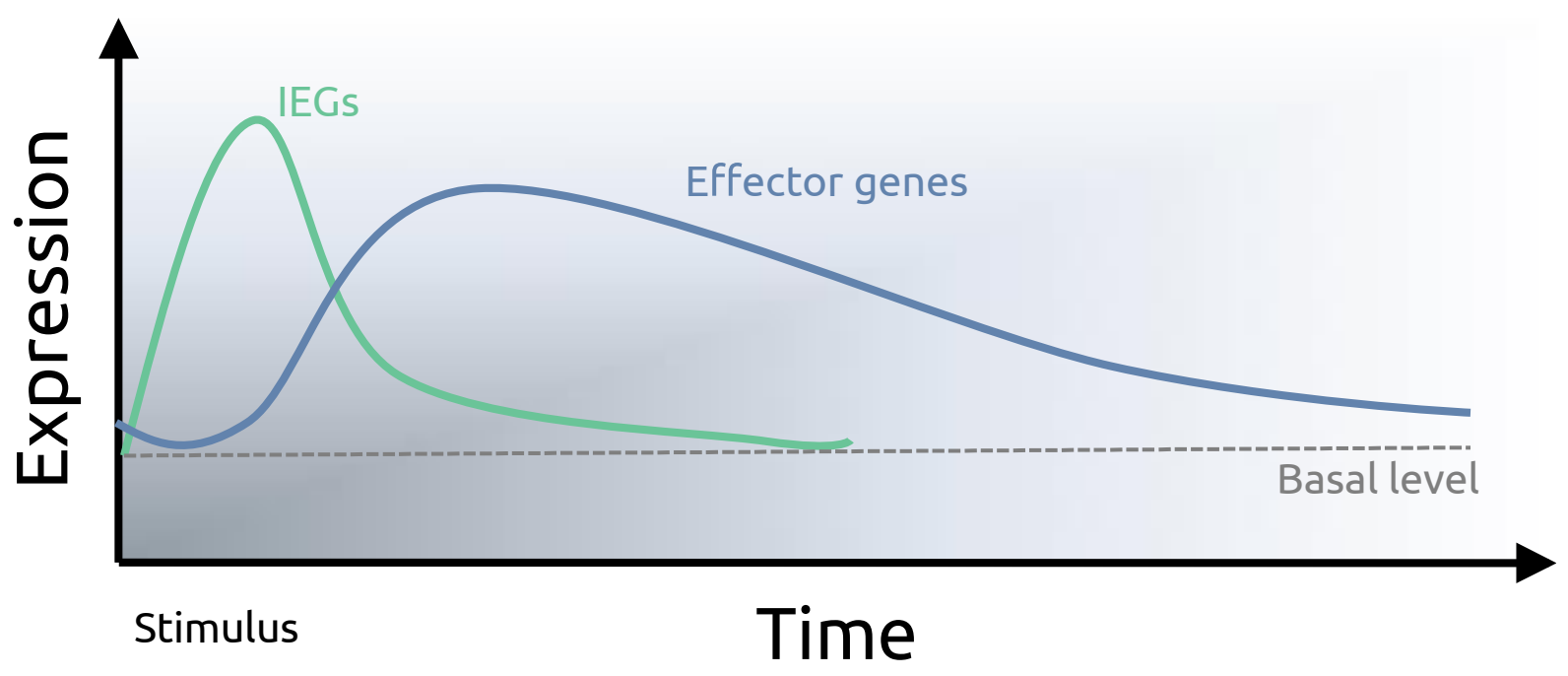

Figure 1-1: Schematic overview of stimulus-dependent gene expression. IEG, Immediate early gene.

It has been shown that novelty, i.e. the presentation of a novel environment can act as such a stimulus to elicit activity-driven gene expression in a neuronal network (Benito et al., 2011; Jones et al., 1996; Moncada and Viola, 2007). However, novelty is likely not to hold the same "relevance" as a stimulus that leads to formation of a memory trace. It is supposed that relevance is mediated by attention, repetition and inborn mechanisms that for example detect life threatening situations or respond to already memorized associations (Atkinson and Shiffrin, 1968).

As outlined before, stimulus-induced gene expression has been shown to correlate with transiently increased histone acetylation at the promoter regions and gene bodies of upregulated genes. Also the timing of increased acetylation coincides with the first waves of expression (Levenson et al., 2004; Morgan and Curran, 1989; Peleg et al., 2010). Notably, the host laboratory could recently show that different gene sets are regulated in response to exploration of a new context and after association of this context with a foot shock (contextual fear conditioning, see 1.1.4). Acetylation of histone $\mathrm{H} 4$ on lysine 12 (H4K12ac) was strongly associated with regulation of the latter gene set, as upregulation of acetylation at this site was impaired in the hippocampus of 16-month-old mice and so was upregulation of this gene set. After administration of SAHA, however, induction of H4K12ac as well as induction of the learning-regulated genes was reinstated, which suggests a causal relationship between $\mathrm{H} 4 \mathrm{~K} 12 \mathrm{ac}$ and learning-induced gene expression. Interestingly, this modification was found enriched not only at the promoter but also throughout the gene bodies of the regulated genes (Peleg et al., 2010). 


\subsubsection{The Histone Acetyltransferase Kat2a (Gcn5)}

Gcn5 was the first protein described to exhibit acetyltransferase activity (Brownell et al., 1996) and is one of the best studied HATs to date (when all homologs from various species from yeast to human are considered). To account for the fact that almost all HATs discovered since then also have non-histone proteins as targets for acetylation, a group of leading researches in the field has recently proposed a new nomenclature for chromatin-modifying enzymes (Allis et al., 2007). Accordingly, all HATs have been renamed as serially numbered KATs (K-Lysine-acetyltransferase), also adding letter indices to account for orthologs in different clades, which for example makes Gcn5's new name for mammals Kat2a (formerly known as Gcn5l2). In this thesis the new name is used, as it is now also the official gene symbol. However, I will refer to histone acetyltransferases as HATs.

The diverse HAT family members are further divided into subfamilies according to sequence homology. Like its close homolog Kat2b (Pcaf), Kat2a belongs to the GNAT subfamily of HATs. Other well studied HAT subfamilies are the Kat3a/Kat3b subfamily or the MYST subfamily, of which some have described functions in the brain and/or cultured neurons (reviewed in Stilling and Fischer, 2011).

The murine Kat2a gene is positioned on chromosome 11. According to the most recent official mouse genome assembly by the genome reference consortium (GRC), the Kat2a gene harbors 18 exons, which is in contrast to the annotation shown in Lin et al., 2008, Figure 1A. Two protein-coding and two non-coding transcripts are expressed from this locus, where the two protein isoforms differ only by the insertion of one amino acid ( $423 \mathrm{~A})$ in a protein region that is not known to be part of any functional domain ${ }^{2}$. The murine Kat2a protein shares $97 \%$ of its amino acids with human Kat2a and both exhibit the same domain structure, as shown in Figure 1-2. One interesting feature is the existence of a bromodomain, which allows Kat2a to bind to pre-acetylated lysine residues. This may already point to a function of Kat2a, where Kat2a is not required for basal (or primary) acetylation but is rather binding to these and further adds additional (secondary), potentially stimulusdependent, acetyl groups to nearby histone tails. This notion is support by evidence for Kat2a binding H4K16ac, a site known to be acetylated in a basal cellular state and to not respond to neuronal activity (Dion et al., 2005; Owen et al., 2000; Peleg et al., 2010; Schiltz et al., 1999).

Like most HATs Kat2a exerts its function in multi-protein complexes. In mammals, Kat2a is a component of at least two different complexes that can either comprise Kat2a or Kat2b in a mutually exclusive manner. While the SAGA complex preferentially acetylates $H 3$, the ATAC complex prefers to acetylate $\mathrm{H} 4$ sites (Anamika et al., 2010). In fact, Kat2a has been shown to preferentially acetylate H3K14 and H4K12 in multiple species (Bu et al., 2007; Ciurciu et al., 2006, 2008; Guelman et al., 2009; Hargreaves et al., 2009; Martinez et al., 2001). Interestingly, the yeast homolog Gcn5p was also found to be preferentially associated with the gene bodies of stressinduced genes (Gunderson and Johnson, 2009; Johnsson and Wright, 2010; Johnsson et al., 2009),

\footnotetext{
${ }^{2}$ source: Ensembl release 70
} 
which suggests role in transcriptional elongation and is in line with mouse data (Hargreaves et al., 2009; Peleg et al., 2010).

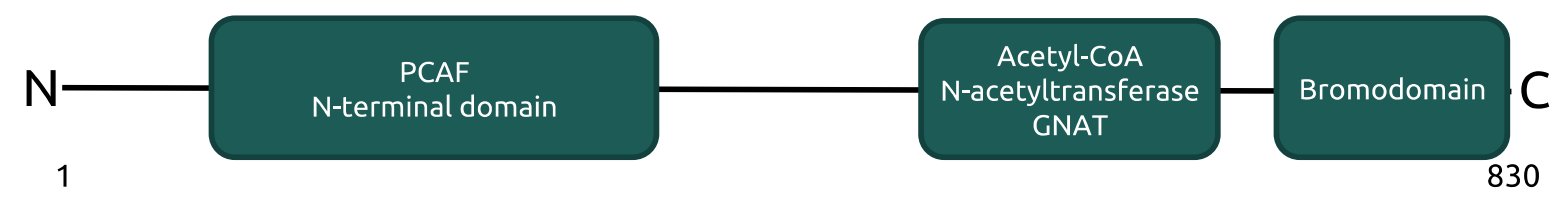

Figure 1-2: Domain structure of the Kat2a protein. Adapted from Interpro database.

Using IN SITU hybridization technology, expression of Kat2a in the murine brain has been found by the brain map project of the Allen Brain Institute to show strong signal in the hippocampal formation (Figure 6-1) (Lein et al., 2007). This is the only data currently available for Kat2a in the adult cerebrum. 


\subsection{Objectives}

\subsubsection{The role of Kat2a in transcription during learning and memory}

Loss of histone-acetyltransferase function is suspected to affect memory consolidation negatively. Among the HATs, only Kat3a (Cbp), Kat3b (p300) and Kat2b (Pcaf) have been tested in this respect. Though Kat2a was the first discovered HAT, its function has only been described in the developing embryonic brain and it has not yet been implicated in learning and memory processes. In fact, though Kat2a is associated with stimulus-dependent transcriptional activation in somatic cells, little is known about its expression in neurons and its function and targets in the adult brain are entirely unexplored.

The aim of the presented project was to determine a putative function of Kat2a in the adult mouse brain. In a first step, expression levels of Kat2a in different brain regions were examined. Furthermore, the role of Kat2a in learning and memory-related behavior was analyzed using lossand gain-of-function approaches. In order to correlate a potential behavioral phenotype with electrophysiological properties of the hippocampus, investigation of long-term potentiation was carried out. To confirm stimulus-dependent transcriptional regulation by Kat2a and to identify potential target genes will help to further establish chromatin plasticity as a key process in governing regulation of neuronal gene expression and increase our knowledge of the cellular and molecular mechanisms underlying learning and memory.

\subsubsection{Histone acetylation and transcription in the aging hippocampus}

Though brain aging goes along with massive transcriptional changes, a detailed, homogenous picture of the transcriptome of the aging mouse hippocampus, especially towards the end of an individual's life span, has not been drawn yet. Accumulating evidence suggests that chromatinrelated mechanisms may be involved in the regulation of transcriptional aging. However, data on the contribution of histone acetylation remain incomplete.

The aim of this project was to extend our knowledge about transcriptional programs associated with the advanced aging and to investigate a potential role of histone acetylation in this process. First, enzymatic activities of HATs and HDACs as well as histone acetylation levels were assessed in 24-month-old mice and mice older than 28 months. Since there is extensive evidence for beneficial effects of HDAC inhibition on cognition in health, aging and disease, it can be reasoned that oral SAHA administration would exert a positive influence on memory formation of mice at an advanced age. Finally, we wanted to comprehensively determine the transcriptional signature of the aging hippocampus to draw conclusions about the processes responsible for ageassociated memory impairment. 


\section{Materials and Methods}

\subsection{Animals and Tissue Isolation}

\subsubsection{Animals}

All animals used in this study were of the species Mus musculus (the common house mouse). Wild type mice were of the inbred strain C57Bl6/J, unless stated otherwise. Specific pathogen free (SPF) C57Bl6/J wild type mice were obtained from Janvier SAS. Mice were kept in individually ventilated cages (IVC, $32 \times 16 \times 14 \mathrm{~cm}$, Techniplast) at the certified, access restricted animal facility of the European Neuroscience Institute, maintained by professional animal caretakers. All procedures described, were performed according to protocols approved by the Lower Saxony State State Office for Consumer Protection and Food Safety (LAVES, Reference number G12.780).

To obtain Kat2a conditional knockout (cKO) mice that carrying a heterozygous "floxed" allele of Kat2a (originally described as Gcn $5^{\text {flox }}$, officially recognized as Kat2atm3.2Roth and hereafter named Kat2 $\left.a^{\mathrm{f} /+}\right)^{3}$ on a 129/Sv-C57Bl/6-mixed background (Lin et al., 2008) were crossed to mice carrying a transgenic construct for expressing the Cre recombinase under control of the CamKIla promoter on a C57BL/6J background (officially recognized as Tg(CamKIla-cre)159Kln, hereafter named CamKIICre ${ }^{\text {tg }}$ ) (Minichiello et al., 1999). Mice carrying the floxed Kat2a allele homozygously and additionally the CamKIla-Cre transgene are cKO mice (hereafter designated as Kat2 $a^{\mathrm{f} / \mathrm{fitg}}$ ), with homozygous recombination of the two loxP sites only in excitatory forebrain neurons resulting in loss-of-function Kat2a alleles restricted to these cell types. The Cre-loxP system is described visually in Figure 2-1A.

Unless otherwise stated, young adult mice at the age of 3 to 5 months were used for behavioral and molecular analyses. Male and female mice were used in the different groups where appropriate to avoid gender bias.

\footnotetext{
${ }^{3}$ According to the original description exons 3 to 18 (of 19) are flanked by the loxP sites. However, Kat2a has only 18 exons according to the most recent genome assembly. For simplicity, it can be agreed that the loxP sites are integrated after exon 2 and before the last 3' exon. Expressed this way, validity is preserved in any case.
} 

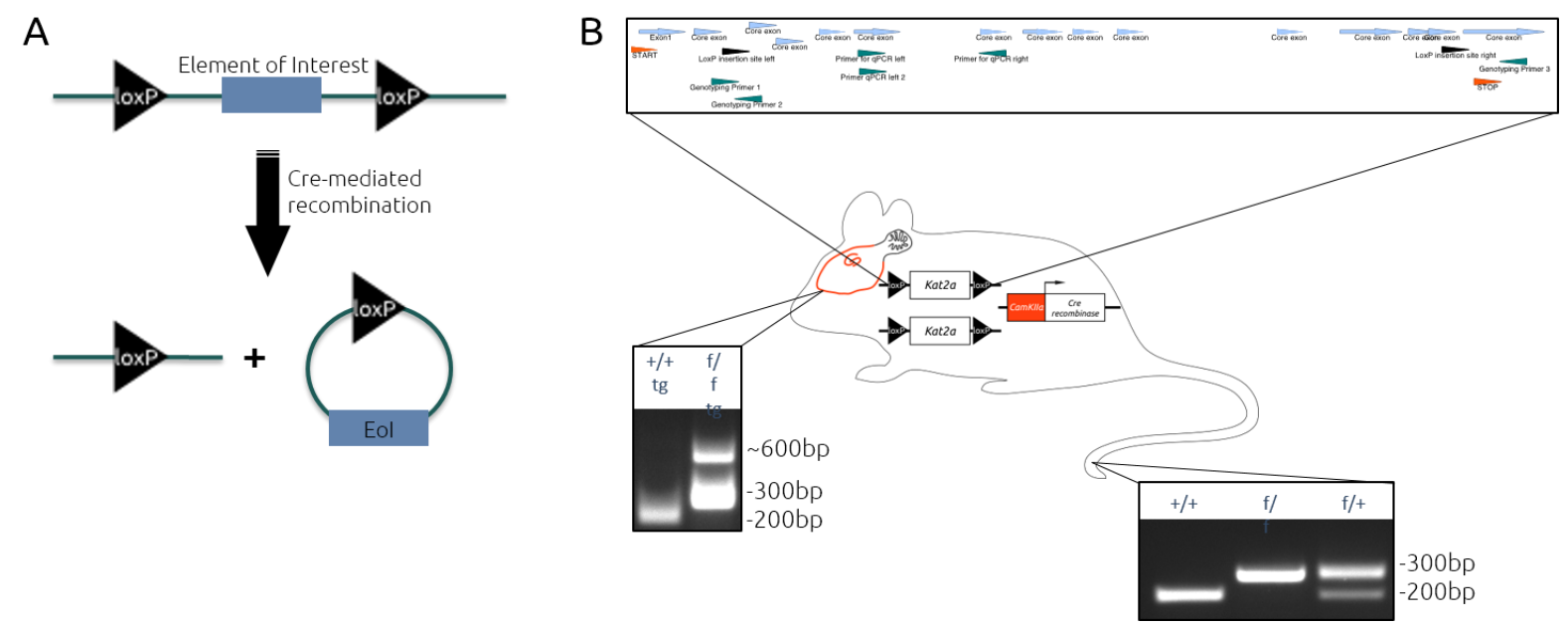

Figure 2-1 The Kat2a-cKO mouse is based on the Cre-loxP system. A DNA element of interest (Eol) is said to be "floxed" if flanked by in-tandem loxP recognition sites for the Cre recombinase. The result of the recombination is loss of the Eol (A). Structure of the floxed Kat2a locus and genotyping results from forebrain (right) and tail tissue (left). The Cre recombinase is expressed under CamKIla promoter, which is active in excitatory forebrain neurons (orange) (B).

\subsubsection{Genotyping}

Genomic DNA was extracted from cut tail tips of mice after weaning using Direct PCR DNA Extraction Reagent (mouse tail) (Viagen) according to the instructions manual. PCR reaction took place in thin-walled $0.2 \mathrm{ml}$ test tubes on a Mastercycler ep gradient S (Eppendorf) Genotyping for the Kat2a locus was performed using three primers to distinguish between wildtype (210 bp PCR product), floxed (310 bp) and recombined alleles ( $600 \mathrm{bp}$ ) as shown in Figure 2-1B (see also Lin et al., 2008). Presence of the CamKII-Cre transgene was tested in a different PCR. Primer sequences for all reactions in this study are given in Appendix 6.4. Reaction mixes and cycling conditions are given in Table 2-1 and Table 2-2, respectively. PCR products were loaded on agarose gels for sizing and visualization as described in 2.4.2.1.3.

Table 2-1: Reaction mixes for genotyping PCRs

\begin{tabular}{llr}
\hline Kat2a genotyping & Volume & CamKII-Cre genotyping \\
\hline Genomic DNA solution & $1 \mu \mathrm{l}$ & Genomic DNA solution \\
Dream-Taq Buffer 10x & $2.5 \mu \mathrm{l}$ & Dream-Taq Buffer 10x \\
dNTP Mix (10 mM each) & $2 \mu \mathrm{l}$ & dNTP Mix (10 mM each) \\
Primer Kat2a-GT_fwd & $0.5 \mu \mathrm{l}$ & Primer Cre-GT-fwd \\
Primer Kat2a-GT_rev & $0.5 \mu \mathrm{l}$ & Primer Cre-GT_rev \\
Primer Kat2a-GT_rev2 & $0.5 \mu \mathrm{l}$ & - \\
Dream Taq (5 u/ $/ \mathrm{ll})$ & $0.2 \mu \mathrm{l}$ & Dream Taq $(5 \mathrm{u} / \mu \mathrm{l})$ \\
$\mathrm{ddH}_{2} \mathrm{O}$ & ad $25 \mu \mathrm{l}$ & $\mathrm{ddH}_{2} \mathrm{O}$ \\
\hline
\end{tabular}


Table 2-2: Cycling conditions for both genotyping PCRs.

\begin{tabular}{llc}
\hline Temperature & \multicolumn{2}{l}{ Duration [s] } \\
\hline 95 & 300 & \\
\hline 95 & 30 & 34 \\
57.5 & 30 & cycles \\
72 & 60 & \\
\hline 72 & 300 & \\
4 & $\infty$ & \\
\hline
\end{tabular}

\subsubsection{Tissue Isolation}

\subsubsection{Tissue Isolation for Extraction of Biological Macromolecules}

Mice were sacrificed by cervical dislocation. The following steps were performed on a metal plat resting on an ice bed. The skull was opened and skull bones removed. The brain was unhinged and tissue was dissected to isolate different brain regions. For retrieval of hippocampal subfield tissue (CA1, CA3 and DG), dissection was performed as described previously (Hagihara et al., 2009; Hörtnagl et al., 1991) in PBS using binocular magnification. The isolated tissue pieces were collected in $1.5 \mathrm{ml}$ test tubes, snap-frozen in liquid nitrogen and kept at $-80{ }^{\circ} \mathrm{C}$ until use.

\subsubsection{Tissue Isolation for Immunohistochemistry}

Mice were anesthetized by intraperitoneal (IP) injection of $250 \mathrm{mg} / \mathrm{kg}$ (bodyweight) Avertin (tribromoethanol, Sigma) and it was waited until full anesthesia occurred. Surgical tolerance was tested checking for whisker, cornea and toe-pinch reflexes, respectively. Only then, mice were transcardially perfused with PBS to wash out the blood, followed by perfusion with $4 \%$ freshly prepared PFA. After fixation the brain was isolated as described above and post-fixed in 4\% PFA over night at $4{ }^{\circ} \mathrm{C}$. Then, PFA was replaced by a $30 \%$ sucrose solution and brains were kept until they sunk to the bottom of the falcon. Sucrose solution was discarded, residual solution dried away and brains frozen on tinfoil over liquid nitrogen. Brains were then embedded in tissue tek medium and cut in a CM1850uv cryostat (Leica Biosystems) at $-20^{\circ} \mathrm{C}$ into sections with a thickness of $30 \mu \mathrm{m}$. Sections were collected in sterile PBS supplemented with Penicillin/Streptavidin solution and kept at $4{ }^{\circ} \mathrm{C}$. PBS was later replaced by cryo-protectant solution (containing $30 \% \mathrm{w} / \mathrm{v}$ sucrose, $1 \% \mathrm{w} / \mathrm{v}$ PVP$40,30 \% \mathrm{v} / \mathrm{v}$ ethylene glycol in $0.2 \mathrm{M}$ phosphate buffer $\left[2.448 \% \mathrm{w} / \mathrm{v} \mathrm{Na} \mathrm{NPO}_{4} \cdot 2 \mathrm{H}_{2} \mathrm{O}, 0.624 \% \mathrm{w} / \mathrm{v}\right.$ $\left.\mathrm{NaH}_{2} \mathrm{PO}_{4} \cdot 2 \mathrm{H}_{2} \mathrm{O}\right]$ ). Immunohistochemistry was performed as described in 2.4.2.3.2.

\subsubsection{Stereotaxic injection of Adeno-associated virus}

Adeno-associated virus (AAV) for IN VIVO expression of Kat2a was generated in collaboration with Dr. Sebastian Kügler's laboratory. The final titer of soluble viral particle aliquots was determined to be $5.4 \times 10^{8}$ transduction units per microliter (TU/ $\left.\mu \mathrm{l}\right)$. GFP-AAV served as control virus. The viral titer was determined to be $4 \mathrm{e} 10^{8} \mathrm{TU} / \mu \mathrm{l}$. Viral particles were diluted to $1.0 \mathrm{e} 10^{8} \mathrm{TU} / \mu \mathrm{l}$ in sterile PBS and $1 \mu \mathrm{l}$ was injected into each hemisphere of anesthetized animals mounted in a digital stereotaxic manipulator (Leica Microsystems) using a surgical driller (Fordom) for skull penetration and a 
microsyringe pump (World Precision Instruments) with an attached glass capillary for injection at a rate of $300 \mathrm{nl} / \mathrm{min}$. Surgical-tolerance anesthesia was achieved as described in 2.1.3.2. Coordinates for injection were (relative to bregma): anteroposterior $-1.75 \mathrm{~mm}$, lateral $-1 \mathrm{~mm}$, dorsoventral $2 \mathrm{~mm}$, thereby targeting DG. After surgery the wound was closed using tissue glue (Histoacryl $\circledast$, Braun) and animals were kept on a warming plate until wake-up.

\subsection{Pharmacological Inhibition of HDAC Activity}

\subsubsection{Injection of Suberoylanilide Hydroxamic Acid in Young Mice}

Young adult wildtype mice ( 3 months old) were injected with either SAHA solution ( $0.5 \% \mathrm{w} / \mathrm{v}$ SAHA, $35 \% \mathrm{DMSO}, 0.9 \% \mathrm{NaCl}$ in $\mathrm{ddH}^{2} \mathrm{O}$, sterile filtered) ${ }^{4}$, or vehicle (35\% DMSO, $0.9 \% \mathrm{NaCl}$ in $\mathrm{ddH}_{2} \mathrm{O}$, sterile filtered) every 24 hours for 7 consecutive days and sacrificed either $1,3,8$ or 24 hours after the last injection.

\subsubsection{Oral Administration of Suberoylanilide Hydroxamic Acid in Aged Mice}

Aged wildtype mice (23 months old) were fed with either $50 \mu$ g SAHA per $g$ body weight (10\% SAHA in DMSO mixed with $200 \mathrm{mg}$ peanut butter) or vehicle (equivalent amount of DMSO with $200 \mathrm{mg}$ peanut butter) every 24 hours for 21 consecutive days.

\subsection{Behavioral Analyses}

If not otherwise stated, all behavioral experiments were digitally recorded and analyzed using the Videomot2 tracking system (Versions 7.02, TSE Systems). Experimenters were blind to the genotype where possible. Where necessary, experimental designs are described in the following and displayed together with their respective figure in the Results section. For all behavioral experiments mice were habituated to the testing room: male mice were kept individually at least one week before the start of the first experiment, while female mice were kept in groups of 2 to 5 animals.

\subsubsection{Open Field}

Animals were placed individually in a uniform-grey plastic arena (50 x $50 \mathrm{~cm}$, walls were $40 \mathrm{~cm}$ high) and allowed to explore the arena for 5 minutes. The room was lit by passive illumination to avoid shading differences within the arena. A virtual $4 \times 4$ grid was drawn over the arena and the 4 inner tiles were defined as center region of the arena. Measured values included total distance, average speed and total time spent in center region vs. total time in surrounding regions.

\subsubsection{Rotarod Performance Test}

Motor function and performance was tested on the TSE RotaRod system. Animals were placed in individual chambers on the rotating rod and habituated for four trials at $10 \mathrm{rpm}, 3$ minutes each.

\footnotetext{
${ }^{4}$ Final concentration (SAHA) $=5 \mu \mathrm{g} / \mu \mathrm{l}, 50 \mu \mathrm{g}$ SAHA per g [body weight] injected, e.g. $300 \mu \mathrm{l} \mathrm{for} \mathrm{a} 30 \mathrm{~g}$ mouse
} 
Falling animals were placed back on the rod to continue training. Aversive stimuli (electric foot shock) were not used. During four testing trials, rotation speed was linearly increasing from 5 to $40 \mathrm{rpm}$ for 3 minutes and kept at $40 \mathrm{rpm}$ for another 1 minute. Time between all trials was 6 to 12 hours. Time until fall was recorded by the supplier's software for each animal during all testing trials.

\subsubsection{Elevated Plus Maze}

Animals were placed individually in a uniform-grey plastic arena consisting of two non-walled (open) and two walled (closed) arms (10 x $40 \mathrm{~cm}$, walls were $40 \mathrm{~cm}$ high). Time spent in open vs. closed arms was measured.

\subsubsection{4-armed Cross Maze-Exploration Test}

Spatial working memory was tested in a uniform-grey plastic arena consisting of four numbered,

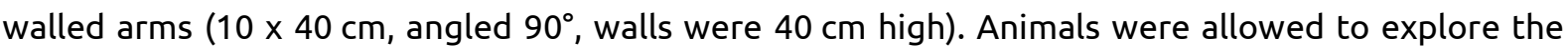
arena for 10 minutes. Mouse behavior was digitally recorded and analyzed using the TSE Videomot2 tracking system (Version 7.02). The sequence of arm entries was extracted and successful trials were counted.

A successful trial was defined as entering an arm that was not entered in one the last three trials. If a mouse would enter arms in random order, the fraction of successful trials out of all trials would be

$$
\frac{4}{4} \times \frac{3}{4} \times \frac{2}{4} \times \frac{1}{4}=0.09375
$$

or $9.375 \%$ ('chance level').

\subsubsection{Novel object recognition}

Animals were habituated individually to a uniform-grey plastic arena $(50 \times 50 \mathrm{~cm}$, walls were $40 \mathrm{~cm}$ high) for 5 minutes on two subsequent days. Animals were then further habituated to two equal objects (objects $A$ and $A$ ) placed in opposing corners of the arena for 5 minutes on the next to days. On day 5 objects $A$ and $A$ were exchanged by two new but equal objects (objects $B$ and $B$ ) and animals were allowed to explore the objects for 5 minutes. Then, mice were sent back to their home cages for 5 minutes (for short-term memory assessment, STM) and reintroduced to the arena after one object was exchanged (objects $B$ and $C$ ). After 24 hours object $C$ was exchanged for object $D$ for long-term memory assessment (LTM). Number and duration of object contacts were measured by the experimenter. Mice that did not contact any of the two objects during a session or only showed summed interest for less than $1 \mathrm{~s}$ were excluded from the analysis of this test.

Object preference was defined as

$$
\frac{\operatorname{time}(B)}{\operatorname{time}(B)+\operatorname{time}(C / D)}
$$


,where time is the summed duration spent with the object.

For comparison of vehicle-treated vs. SAHA-treated 24-month-old-mice, a similar setting was used. However, mice were habituated for only one day in an arena that was $100 \times 100 \mathrm{~cm}$ with $20 \mathrm{~cm}$ high transparent walls. Objects $A$ and $A$ were used for training on day 2 and objects $B$ and $C$ were introduced after a 5 min or 24 hours stay in the home cage, respectively.

\subsubsection{Fear conditioning}

Fear conditioning was performed, recorded and analyzed on the NIR Video Fear Conditioning system (Med Associates Inc.) using Video Freeze ${ }^{T M}$ software. Mice were placed in a sound-protected box supplied with white noise and allowed to explore the new context, while baseline freezing was monitored. After 3 minutes, an electrical foot shock ( $0.7 \mathrm{~mA}, 2$ seconds) was delivered through the grid floor. 24 hours later, animals were reintroduced to the cage and contextual freezing behavior was recorded for 3 minutes. Freezing was counted in linear analysis mode, if the Motion Index was below a threshold of 50 for longer than 30 frames (at 30 frames per second).

\subsubsection{Morris Watermaze}

Animals were placed in a circular pool (1.2 m diameter) containing opaque water at ambient temperature $\left(20-22^{\circ} \mathrm{C}\right.$ ) and a platform submerged $1 \mathrm{~cm}$ below the water surface. The pool was equipped with four visual cues for orientation. On each day mice were allowed to swim on four trials, each starting from a different starting point. Escape latency was measured as the time the mice needed to find the platform. If they did not find it within 1 minute, mice were guided to the platform by the experimenter. On the day of probe testing, the platform was removed and mice were allowed to swim for 1 minute. A virtual grid of 4 quadrants was drawn over the pool surface and time spent in each quadrant was measured, the target quadrant being the one, where the platform had been placed during training. The percentage of time spent in each quadrant (chance level $=1 / 4=25 \%$ ) and the number of crossings of as well as time spent in the platform region was calculated.

In a visual form of the test described above, the submerged platform was flagged with a visual cue and trials were performed for four days with no subsequent probe test.

\subsubsection{Novelty exposure}

Male mice were individually exposed to a uniform-grey plastic arena $(50 \times 50 \mathrm{~cm}$, walls were $40 \mathrm{~cm}$ high) containing four objects in each corner of the arena, different in shape, size, color and material. Mice were allowed to explore the objects for 15 minutes while movements were monitored. One hour after they were sent back to their home cages, mice were sacrificed and tissue was isolated as described in 2.1.3.1. Naïve mice taken directly from their home cage served as controls. 


\subsection{Molecular Analyses}

For all protocols, all steps were performed at RT, unless stated otherwise.

\subsubsection{Extraction of Biological Macromolecules}

\subsubsection{Extraction of Genomic DNA}

Genomic DNA was extracted only for the use of genotyping as described in 2.1.2.

\subsubsection{Extraction of $R N A$}

In general, all materials and workbenches used for RNA extraction were wiped with RNaseZAP (Sigma-Aldrich). RNA was extracted according to the SOP of the Transcriptome Analysis Laboratory (TAL). In brief, flash-frozen tissue was homogenized on ice with several pestle strokes in $0.5 \mathrm{ml}$ of TRI Reagent (Sigma-Aldrich) After addition of another $0.5 \mathrm{ml}$ of TriReagent and $5 \mathrm{~min}$ incubation at room temperature (RT) the dissociated homogenate was mixed with $300 \mu \mathrm{l} \mathrm{of} \mathrm{CHCl}_{3}$ and incubated for $15 \mathrm{~min}(\mathrm{RT})$ followed by centrifugation at $12,000 \times g\left(4^{\circ} \mathrm{C}\right)$. The upper aqueous phase was transferred to a new tube, mixed with $500 \mu$ isopropanol and incubated at $-20^{\circ} \mathrm{C}$ for at least 1 hour for precipitation. RNA was precipitated by centrifugation at $12,000 \mathrm{~g}$ for 30 minutes $\left(4^{\circ} \mathrm{C}\right)$. The pellet was washed twice with $1 \mathrm{ml}$ of $75 \%$ ethanol (centrifugation after washing steps: $12,000 \times g$, $5 \mathrm{~min}, 4^{\circ} \mathrm{C}$ ). The washed pellet was dissolved in $30 \mu$ of RNase-free water.

For genome-wide methods (RNA-seq, microarray) retrieved RNA was treated with DNase I according to the SOP of the TAL: To the $30 \mu$ l of dissolved RNA $5 \mu$ l of 10x incubation buffer, $1 \mu l$ DNase I and $0.5 \mu \mathrm{lnNaseOUT}{ }^{\mathrm{TM}}$ (all life Technologies) was added and filled up to a final volume of $50 \mu \mathrm{l}$ with RNase free water. Samples were then incubated for $20 \mathrm{~min}$ at $37^{\circ} \mathrm{C}$ followed by addition of an $150 \mu \mathrm{l}$ of RNase free water and $200 \mu \mathrm{l}$ of phenol:chloroform:isoamylalcohol (25:24:1 ratio, Applichem). After vortexing and centrifugation at 12,000 $\times \mathrm{g}$ for $2 \mathrm{~min}$ (RT), the upper aqueous phase was transferred two a new tube and mixed with $20 \mu \mathrm{l}$ of $3 \mathrm{M}$ sodium acetate pH 4.8, $200 \mu \mathrm{l}$ isopropanol and $1 \mu$ Glycoblue (life Technologies). For precipitation samples were kept at $-20 \mathrm{C}$ for at least 1 hour and afterwards centrifuged for $30 \mathrm{~min}$ at 16,100 $\mathrm{g}$. The resulting pellet was washed twice with $1 \mathrm{ml}$ of $75 \%$ ethanol (centrifugation after washing steps: $12,000 \times \mathrm{g}, 5 \mathrm{~min}, 4^{\circ} \mathrm{C}$ ). The pellet was then redissolved in $30 \mu \mathrm{l}$ of RNase-free water.

\subsubsection{Extraction of Proteins}

Protein extracts from subcellular compartments were obtained using two different methods. In general, flash-frozen tissue was homogenized on ice with several pestle strokes under buffered and protease-inhibited conditions. To extract proteins from cytosolic as well as membrane and nuclear proteins the ProteoExtract@ Subcellular Proteome Extraction Kit (Merck Chemicals / Calbiochem) was used according to the manufacturer's recommendations.

To separate cytoplasmic from nuclear protein fractions tissue was homogenized in $0.5 \mathrm{ml}$ TX Buffer (1\%v/v Triton X 100, $150 \mathrm{mM} \mathrm{NaCl}, 50 \mathrm{mM}$ Tris HCl, 2 mM EDTA in PBS, pH 7.4, supplemented with Complete Protease Inhibitor Cocktail, Roche Applied Science) and incubated for $15 \mathrm{~min}$ on a 
rotation wheel $(15 \mathrm{rpm})$ at $4{ }^{\circ} \mathrm{C}$. After centrifugation $\left(10 \mathrm{~min}, 4^{\circ} \mathrm{C}, 400 \mathrm{xg}\right.$ ), supernatant was collected as cytoplasmic fraction. The remaining nuclear pellet was dissolved in TX Buffer supplemented with $1 \% \mathrm{SDS}$, incubated in for $5 \mathrm{~min}$ on a rotation wheel $\left(15 \mathrm{rpm}, 4^{\circ} \mathrm{C}\right)$ and sent to an ultrasound bath (Bioruptor ${ }^{\circledR}$, Diagenode) for 15 cycles ( $30 \mathrm{~s}$ ON / 30 s OFF). After centrifugation $\left(10,000 \times \mathrm{g}, 10 \mathrm{~min}, 4^{\circ} \mathrm{C}\right)$, the supernatant was collected as nuclear fraction, the pellet was discarded.

\subsubsection{Detection of Biological Macromolecules}

\subsubsection{Detection of DNA}

\subsection{DNA Quantity Measurements and Quality Control}

DNA concentration and 260/280 nm values were determined using a Nanodrop 2000 (Thermo Scientific). For ChIP-seq, concentration and quality (fragmented DNA size) was measured using High Sensitivity DNA kits with Agilent's 2100 Bioanalyzer (Agilent Technologies) according to the supplier's protocols.

\subsubsection{2. $\quad P C R$ Amplification}

For PCR amplification of gDNA for genotyping purposes see 2.1.2

For PCR amplification of cDNA fragments for molecular cloning purposes see 2.4.3.1

\subsection{DNA Gel Electrophoresis and Purification}

For DNA sizing and visualization, agarose-gel electrophoresis was used. $0.8-2 \% \mathrm{w} / \mathrm{v}$ agarose in $1 \mathrm{x}$ TAE buffer ( $40 \mathrm{mM}$ Tris-acetate, $1 \mathrm{mM}$ EDTA in $\mathrm{ddH}_{2} \mathrm{O}$ ) was boiled until complete solution, supplemented with $2-4 \mu \mathrm{l}$ of ethidium bromide stock solution $(10 \mathrm{mg} / \mathrm{ml})$ and poured to electrophoresis chambers. Separation was achieved in electrophoresis chambers submerged in TAE buffer at constant voltage (100 V). For genotyping, a green loading dye was already a component of the PCR Mastermix. For all other PCRs (see 2.4.3.1) 6x loading dye (Thermo Scientific) was added to the sample to a final concentration of $1 \mathrm{x}$. For sizing purposes, a reference size marker (100 bp ladder or 1000 bp ladder, Thermo Scientific) was loaded on each gel.

For purification of DNA fragments from gel, the piece of interest was cut out and subjected for treatment with the QIAquick Gel Extraction Kit (Qiagen) according to the manufacturer's manual.

\subsection{DAPI Staining}

DAPI (life Technologies) stock solution ( $5 \mathrm{mg} / \mathrm{ml}$ ) was diluted 1:10,000 in the secondary antibody solution and incubated as described in 2.4.2.3.2.

\subsection{DNA Sequencing}

For routine Sanger sequencing of amplified constructs and PCR products, purified DNA ${ }^{5}$ was prepared according to Seqlab recommendations ${ }^{6}$, picked up by Seqlab service and sequenced using

\footnotetext{
${ }^{5}$ QIAquick PCR / Gel Purification kit (Qiagen) for PCR products, purification of plasmids is described in 2.4.3.4

${ }^{6}$ Recommended amount of DNA was mixed with a single specific primer
} 
Extended HotShot sequencing. Lasergene Seqman (Dnastar) was used for sequence inspection and alignment.

ChIP-seq was performed for genome-wide H3K9ac occupation studies. ChIP samples were purified using ChIP DNA Purification Kit (Active Motif) and shipped to Fasteris SA. There DNA concentration is verified by fluorometry using the Qubit (life Technologies). The libraries were prepared as described by Illumina in their ChIP-seq library preparation kit. Briefly, the DNA is end-repaired to produce phosphorylated blunt ends, A-tailed to get a 3' protruding A residue and ligated with Illumina's adapters. The final library obtained by PCR amplification (15 cycles) was verified on an Agilent 2100 Bioanalyzer (Agilent Technologies) for size distribution and concentration. The libraries were analyzed on a $1 \times 50$ run on the HiSeq instrument ${ }^{7}$. Data analysis for quality control, basic statistics and generation of region specific plots was performed as described in 2.6.4.

\subsubsection{Detection of $R N A$}

\subsection{RNA Quantity Measurements and Quality Control}

RNA concentration and 260/280 nm values were determined using a Nanodrop 2000 (Thermo Scientific). For genome-wide assays (RNA-seq, microarray) concentration and quality (RNA integrity number, RIN) was measured using RNA 6000 nano or 6000 pico kits on an Agilent 2100 Bioanalyzer (Agilent Technologies) according to the supplier's protocols.

\subsection{CDNA Synthesis}

cDNA synthesis was performed using the First Strand Synthesis kit (Roche Applied Science) according to the manufacturer's protocol. In brief, $1 \mu$ g of RNA was used as input and denatured for 10 min at $65^{\circ} \mathrm{C}$. Then first strand CDNA synthesis was performed using random hexamer primers.

\subsubsection{3. $\quad q R T-P C R$}

For expression analysis by quantitative real-time PCR (qRT-PCR), CDNA was diluted 1:10 in PCRgrade $\mathrm{H}_{2} \mathrm{O}$ and amplified using the LightCycler ${ }^{\circledR} 480$ System including LC480 Mastermix in association with the Universal ProbeLibrary (UPL) system (Roche Applied Science). Primers and probes were selected using the UPL Assay Design Center. Murine housekeeping gene Hprt served as internal reference. I each run (96-well plate) all qRT-PCR samples were measured in duplicates.

\subsection{DNA Microarray}

DNase I treated RNA or frozen tissue was handed to the TAL. There, RNA extraction, quality control, cDNA synthesis, mono-color Cy3-labeling and hybridization to whole mouse genome microarray chips were carried out according to TAL-own SOPs. In brief, total RNA was labeled with Cy3 according to Agilent's Low RNA Input Fluorescent Linear Amplification Kit and later hybridized to Agilent Whole Mouse Genome 4x44K G4122F microarrays according to the manufacturer's protocol. Quantity and Cydye incorporation rates of the generated target material were assessed using a NanoDrop ND-1000. Washes were performed according to the Agilent Technologies SSPE protocol (v2.1) - wash solution 3 was replaced by acetonitrile. After that, scanning was performed using an Agilent G2505B scanner. ${ }^{8}$

\footnotetext{
${ }^{7}$ Protocol description kindly provided by Fasteris SA.

${ }^{8}$ Protocol description kindly provided by the TAL
} 
Data analysis to generate lists of differentially expressed genes (DEGs) was performed as described in 2.6.1.

\subsection{RNA Sequencing}

DNase I treated RNA was quality controlled (see 2.4.2.2.1) and handed to technical assistance (for the Kat2a and aging project) or the TAL (for the aging project). Library preparation and cluster generation for mRNA sequencing was performed according to Illumina standard protocols using the TruSeq RNA Sample Prep Kit v2 and the TruSeq Paired-End Cluster Generation Kit v3-cBot-HS (for PE mRNA-seq) with subsequent use of the corresponding TruSeq Cluster Generation Kit v3-cBot-HS (for SE mRNA-seq). Libraries were quality controlled and quantified using a Nanodrop 2000 (Thermo Scientific), an Agilent 2100 Bioanalyzer (Agilent Technologies) and Qubit (life Technologies). For the sequencing run, TrueSeq SBS kits were used according to Illumina manuals. Data analysis for quality control, basic statistics and generation of lists of differentially expressed genes (DEGs) and comparison of transcript abundance was performed as described in 2.6.2.

\subsubsection{Detection of Proteins}

For all immunological-based detection of proteins commercially available antibodies were used. For the full list of antibodies used in this thesis, please refer to Appendix 6.5.

\subsection{SDS-PAGE and Western Blot}

Protein lysates (see 2.4.1.3) were diluted to $1 \mathrm{\mu g} / \mathrm{\mu l}$ with Laemmli sample buffer (CSH Protocols,

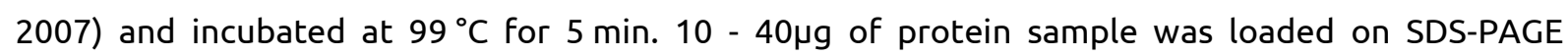
acrylamide gels, consisting of a stacking gel (5\% polyacrylamide [PA]) on top of a separating gel (10 - 15\% PA). All devices for SDS-PAGE and western blot were from Bio-Rad. $5 \mu$ of protein-sizing markers (Thermo Scientific) were loaded on every gel. Gels were submerged in electrophoresis buffer ( $25 \mathrm{mM}$ Tris, $250 \mathrm{mM}$ Glycine, $0.1 \%$ SDS in $\mathrm{ddH}_{2} \mathrm{O}$ ) and electrophoresis was carried out at constant voltage ( $45 \mathrm{~min}$ at $60 \mathrm{~V}$ plus 1.5 hours at $100 \mathrm{~V}$ ).

Afterwards, submerged in transfer buffer (192 mM Glycine, $25 \mathrm{mM}$ Tris- $\mathrm{HCl}$ pH 8.3, 20\% Methanol in $\left.\mathrm{ddH}_{2} \mathrm{O}\right)$, proteins were transferred to a PROTRAN® nitrocellulose membrane $(0.2 \mu \mathrm{m}$ pore size, Whatman) in a cooled device $\left(4^{\circ} \mathrm{C}\right)$ at constant voltage $(45 \mathrm{~V})$ for 12 to 20 hours.

Blotted membranes were incubated in non-fat milk (5\%w/v milk powder (Roth) in TBS [5 mM Tris, $15 \mathrm{mM} \mathrm{NaCl}, \mathrm{pH} 7.6])$ for blocking and incubated with the primary antibody of interest $\mathrm{ON}$ at $4{ }^{\circ} \mathrm{C}$ (antibodies were diluted with in $0.5 \% \mathrm{w} / \mathrm{v}$ milk powder, $0.05 \% \mathrm{v} / \mathrm{v}$ Tween ${ }^{\circledR}-20$ (Roth) in TBS).

Afterwards the membrane was washed 3 times for $5 \mathrm{~min}$ in $0.05 \% \mathrm{v} / \mathrm{v}$ Tween $®-20$ in TBS, incubated with the appropriate (fluorescent) secondary antibody for 30 minutes (dilution as primary antibody) and washed again 3 times. Membranes were stored in TBS until imaging. Membranes were scanned and the results quantified using the Odyssey $®$ Imaging system (Li-cor).

\subsection{Immunohistochemistry and Imaging}

Immunohistochemistry was used to visualize protein abundance and localization in floating coronal and sagittal cryosections of mouse brain tissue that were prepared as described in 2.1.3.2. Sections 
were transferred to 24-well plates (1 section per well) and washed twice with $500 \mu l 9$ of PBS supplemented with $0.2 \%$ Triton X 100 (TX, Roth) for 5 min followed by incubation in $500 \mu$ of blocking solution (5\% goat serum (PAN Biotech), 0.3\% TX in PBS) for 90 min. After blocking, $300 \mu$ of primary antibody solution (prepared in blocking buffer) 10 was added and incubated over night (ON) at $4{ }^{\circ} \mathrm{C}$. Washing was performed with 3 times $500 \mu$ of $1 \%$ goat serum plus $0.2 \%$ TX in PBS for 10 min each. Incubation with $300 \mu$ secondary antibody solution (prepared in blocking buffer) 11,12 for 60 min was followed by 3 washes with $500 \mu$ of $0.2 \%$ TX in PBS and an additional wash in $500 \mu$ pure PBS, all steps under light protected conditions. Sections were dried, mounted on super frost+ glass slides (Thermo Scientific), submerged in polyvinylalcohol (Mowiol@ 4-88, Sigma-Aldrich) covered with a glass cover slip. Slides were kept at $4{ }^{\circ} \mathrm{C}$ until imaging with a confocal microscope (DMIRE2, Leica) or an epifluorescence light microscope (IX70, Olympus). For image acquisition, on the confocal microscope LCS software (v2.6.1, Leica), on the epifluorescence light microscope a digital camera (DP71, Olympus) in combination with Cell F software (Olympus) were used. Image manipulations were done using Image $J(\mathrm{NIH})$ and LCS software (v2.6.1 Leica) was used for fluorescence quantification.

Immunostaining of Kat2a using was done as described above, with the following difference: Sections were incubated in Citrate Buffer (10 mM Tri-sodium citrate (Applichem), 0.1\% TX in $\mathrm{ddH}_{2} \mathrm{O}$ ) at $95^{\circ} \mathrm{C}$ for 10 minutes before submitting to washes and blocking.

\subsection{HAT / HDAC Activity Measurements}

For HAT and HDAC activity measurements commercially available colorimetric assay kits (K332-100 and K331-100, respectively, Biovision) that pick up non-specific activity of enzymatic families were used according to the manufacturer's manuals. $40-120 \mu \mathrm{g}$ of hippocampal nuclear fraction (see 2.4.1.3) were used as enzyme source. Data acquisition for all assays mentioned above was carried out on an Infinite ${ }^{\circledR} 200$ PRO multimode reader (TECAN) using i-control software (v1.9).

\subsubsection{Cloning of AAV constructs}

Cloning of cDNA constructs was used to prepare vectors for virus generation. AAV-ready plasmids were constructed for the following genes, all carrying a MYC-tag: Kat2a, Kat5 (formerly known as Tip60), Kat7 (Myst2 or Hbo1), Hdac2 and Hdac3. Sequenced (see 2.4.2.1.5) and test-digested plasmids of Kat2a and Hdac2 were then used to generate AAV viruses as described in 2.1.4. Kat2a-MYC-AAV was used for further analysis or IN VIVO overexpression (OE).

\subsubsection{Linker-PCR from Mouse Hippocampal cDNA}

To obtain CDNA containing appropriate restriction sites for cloning into the target vector, a linkerPCR was performed in two steps. In the first step, cDNA of interest was amplified with cDNA-

\footnotetext{
${ }^{9}$ All following volumes are given as per well.

${ }^{10}$ For dilutions see 6.5.1

${ }^{11}$ For dilutions see 0

${ }^{12}$ DAPI was added where necessary as described in 2.4.2.1.4
} 
specific forward (fwd) and reverse (rev) primers. ${ }^{13}$ In the second step, the cDNA-specific reverse primer was substituted for a universal reverse primer, used for all cDNA amplifications. ${ }^{14}$ Reaction mixes and cycling conditions are given in Table 2-3 and Table 2-4, respectively. For each PCR 6 reactions were mixed used with different primer annealing temperatures (gradient). PCR products were loaded on agarose gels for sizing, visualization and gel purification of the correctly sized band as described in 2.4.2.1.3. Conditions for linker-PCRs were chosen based on the Phusion High-Fidelity DNA Polymerase (Thermo Scientific) manual and practical experience.

Table 2-3: Reaction mixes for linker-PCRs

\begin{tabular}{|c|c|}
\hline Reagent & Volume \\
\hline $\begin{array}{l}\text { Hippocampal cDNA / } \\
\text { PCR product form step } 1\end{array}$ & $0.5 \mu \mathrm{l}$ \\
\hline Phusion HF Buffer $5 x$ & $10 \mu$ \\
\hline dNTP Mix (10 mM each) & $5 \mu$ \\
\hline $\begin{array}{l}\text { Primer fwd } \\
\text { (depending on target (see 6.4) }\end{array}$ & $1 \mu l$ \\
\hline $\begin{array}{l}\text { Primer rev } \\
\text { (depending on target (see } 6.4 \text { ) }\end{array}$ & $1 \mu$ \\
\hline Phusion (2 u/pl) & $0.5 \mu l$ \\
\hline $\mathrm{dd}_{2} \mathrm{O}$ & ad $50 \mu$ \\
\hline
\end{tabular}

Table 2-4: Cycling conditions for linker- PCRs

\begin{tabular}{llc}
\hline Temperature & Duration [s] & \\
\hline 98 & 45 & \\
\hline 98 & 20 & 10 \\
$52-63$ (gradient) & 30 & cycles \\
72 & 150 & \\
\hline 98 & 20 & 22 \\
$65-72$ (gradient) & 30 & cycles \\
72 & 150 & \\
\hline 72 & 420 & \\
4 & $\infty$ & \\
\hline
\end{tabular}

\subsubsection{Restriction Digestion and Ligation}

Restriction digestion of PCR products and target vector was achieved using FastDigest enzymes EcoRI, Notl, Nhel according to the target (Thermo Scientific). FastDigest Smal (Thermo Scientific) was used to digest final AAV constructs to make sure inverted terminal repeats (ITR) were present.

\footnotetext{
${ }^{13}$ Primers were carrying the necessary $5^{\prime}$ restriction site (EcoRI or Nhel) and vertebrate Kozak sequence (CCACC) \{Kozak 1987 NAR\} on the forward primer and part of the MYC-tag (Amino acid sequence of MYC-tag: EQKLISEEDL) on the reverse primer. The specific reverse primers were lacking the Stop-Codon. The primers were designed in a way to only amplify the protein coding DNA sequence (CDS). Untranslated regions (UTRs) were not amplified.

${ }^{14}$ The universal reverse primer carried the remaining part of the MYC-tag, a Stop-Codon and a restriction site for Notl.
} 
Reaction mixes are given in Table 2-5. Reactions mixes were incubated at $37^{\circ} \mathrm{C}$ for $15-30 \mathrm{~min}$ and digested constructs were sized and retrieved by gel purification as described in 2.4.2.1.3. Reaction mixes and incubations were based on recommendations by the manufacturer and practical experience.

Ligation was carried out using T4 DNA Ligase (Thermo Scientific) according to the supplier's protocol. In brief, target vector and insert were mixed in a ratio of 1:5 and incubated with the ligase at $22^{\circ} \mathrm{C}$ for $10-20 \mathrm{~min}$. The reaction mix is given in Table 2-6. Afterwards, the ligase was inactivated by incubation at $75^{\circ} \mathrm{C}$ for $10 \mathrm{~min}$. Mass of vector to be used was set to $50 \mathrm{ng}$. Masses of inserts were determined according to the following formula:

$$
\operatorname{insert}(n g)=\frac{\operatorname{insert}(k b) \cdot \operatorname{vector}(n g) \cdot 5}{\text { vector }(k b)}
$$

The AAV target vector plasmid, called AAV-6P-NoTB-SEWB (5288 bp), was a kind gift from Dr. Sebastian Kügler's laboratory.

Table 2-5: Reaction mixes for restriction digestion

\begin{tabular}{ll}
\hline Reagent & Volume \\
\hline Target vector or PCR product & $5 \mu \mathrm{l}$ \\
FastDigest Restriction Enzyme & $1 \mu$ l each \\
FastDigest universal buffer & $2 \mu \mathrm{l}$ \\
$\mathrm{ddH}_{2} \mathrm{O}$ & $\mathrm{ad} 20 \mu \mathrm{l}$ \\
\hline
\end{tabular}

Table 2-6: Reaction mixes for ligation digestion

\begin{tabular}{ll}
\hline Reagent & Volume or Mass \\
\hline Digested Target vector & $50 \mathrm{ng}$ \\
Digested PCR product & (according to formula) \\
T4 DNA ligase & $1 \mu \mathrm{l}$ \\
T4 DNA ligation buffer & $1 \mu \mathrm{l}$ \\
$\mathrm{dd}_{2} \mathrm{O}$ & ad $10 \mu \mathrm{l}$ \\
\hline
\end{tabular}

\subsubsection{Bacterial Amplification of DNA Constructs}

Target vectors or ligated constructs were amplified using SURE competent cells (Stratagene / Agilent Technologies). To this end, $0.5 \mu$ of ligation mix was added to provided frozen bacterial cell aliquots and cells were transformed by electroporation with an Electroporator 2510 (Eppendorf). Freshly electroporated cells were added to $1 \mathrm{ml}$ of Lysogeny broth (LB) medium (1\%w/v Tryptone, 
$0.5 \% \mathrm{w} / \mathrm{v}$ yeast extract, $1 \% \mathrm{w} / \mathrm{v} \mathrm{NaCl}$ (all Applichem)) without addition of any antibiotic and

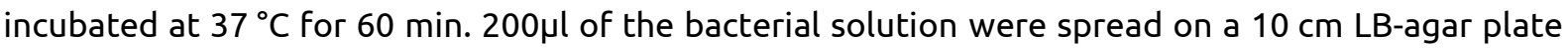
(LB medium $+1.5 \% \mathrm{w} / \mathrm{v}$ agar) containing $100 \mu \mathrm{g} / \mu \mathrm{l}$ of ampicillin (Sigma-Aldrich). Agar plates were incubated at $37^{\circ} \mathrm{C}$ for at least 16 hours and colony-forming clones were selected for further amplification in $5 \mathrm{ml}$ liquid LB medium with ampicillin (as before) for small-scale preparation. Positive clones with correctly amplified sequence and target vector backbone were selected for large-scale production of plasmid DNA.

\subsubsection{Plasmid Purification}

For small-scale preparation, bacterial cells were collected by centrifugation ( $4000 \times g, 10 \mathrm{~min}, 4^{\circ} \mathrm{C}$ ) and plasmid DNA was retrieved using the QuickLyse Miniprep Kit (Qiagen) according to the supplied manual. Plasmid sequencing and test digestion was performed as described in 2.4.2.1.5 and 2.4.3.2, respectively.

Large-scale preparation was achieved by using NucleoBond® PC 2000 kit (Macherey-Nagel) as recommended by the supplier with the following modifications. A 1 I culture was used to harvest bacterial starting material. Harvesting was achieved by centrifugation at $6000 \mathrm{xg}$ for $15 \mathrm{~min}$ at $4{ }^{\circ} \mathrm{C}$ multiple times as only $200-250 \mathrm{ml}$ of culture could be centrifuged in each round; in between supernatant was discarded. Elution of column-bound plasmid DNA was done over night at $4{ }^{\circ} \mathrm{C}$, DNA was precipitated by the use of $18 \mathrm{ml}$ isopropanol and the pellet was dissolved in $3 \mathrm{ml}$ of TE buffer (10 mM Tris, 1mM EDTA (all Applichem)). To further purify DNA, another precipitation step was introduced. DNA was precipitated with 2.5 volumes of cold $100 \%$ ethanol (Roth) and $1 / 10$ volumes of $3 \mathrm{M}$ sodium acetate (Roth) and centrifuged at $10,000 \times \mathrm{g}$ for $30 \mathrm{~min}$ at $4{ }^{\circ} \mathrm{C}$. The pellet was washed with $7 \mathrm{ml}$ of $70 \% \mathrm{v} / \mathrm{v}$ ethanol and dissolved in TE buffer. 


\subsubsection{Chromatin Immunoprecipitation (ChIP)}

ChIP was carried out using the fixation-based (XChIP) Shearing Optimization kit and One Day ChIP kit (Diagenode) according to the manufacturer's recommendations, with the following alterations: Hippocampal tissue was used as starting material and was homogenized in sterile PBS supplemented with Complete Protease Inhibitor Cocktail, Roche Applied Science). Fixation was achieved by incubation of the homogenate with $1 \%$ formaldehyde (final concentration, Applichem) for $10 \mathrm{~min}$ on a rotation wheel (12 rpm, RT). Fixation was stopped by adding $1.25 \mathrm{M}$ Glycine (Applichem) and were then subjected to further processing using kit Buffers A, B, C and D. For chromatin shearing, samples were placed in a Bioruptor ${ }^{\circledR}$ high-energy ultra-sound sonification device (Diagenode) and sonicated for 25 cycles ( 1 cycle $=30 \mathrm{~s}$ ON / $30 \mathrm{~s}$ OFF) with intermediate changes of ice. Immunoprecipitation was achieved by incubation of the antibody-chromatin mix for 1 hour in an ultra-sonic cleaner bath (VWR) at $4{ }^{\circ} \mathrm{C}$. Protein-A-covered agarose beads were blocked with bovine serum albumin for 20 minutes and incubated with the antibody-bead-mix for 60 min on a rotation wheel $\left(12 \mathrm{rpm}, 4^{\circ} \mathrm{C}\right.$ ). The resulting antibody-chromatin-bead complexes were washed twice with $1 \mathrm{x}$ provided ChIP buffer. Proteinase $\mathrm{K}$ digestion was for 1 hour at $55^{\circ} \mathrm{C}$. 


\subsection{Electrophysiological Analyses}

\subsubsection{Measurement of LTP}

Electrophysiological analysis was performed in collaboration with the laboratory of Prof. Dr. Klaus Reymann (DZNE Magdeburg). Hippocampal slices (400 $\mu \mathrm{m}$ thick) were prepared from 4-months-old male Kat2 $d^{f / f}$ or Kat2 $d^{f / f i t g}$ mice. Mice were killed by hyperextension of the neck. After decapitation, the brain was quickly removed and placed into ice-cold artificial cerebrospinal fluid (ACSF) with the following composition (in mM): $\mathrm{NaCl} 124, \mathrm{KCl} 4.9, \mathrm{MgSO}_{4}$ 1.3, $\mathrm{CaCl}_{2} 2.5, \mathrm{KH}_{2} \mathrm{PO}_{4}$ 1.2, $\mathrm{NaHCO}_{3}$ 25.6, Dglucose 10 , saturated with $95 \% \mathrm{O}_{2} / 5 \% \mathrm{CO}_{2}, \mathrm{pH}$ 7.4). Transverse slices were prepared using a tissue chopper with a cooled stage and immediately transferred into a pre-chamber containing $8 \mathrm{ml}$ of permanently carbogen-gasified ACSF for $2 \mathrm{~h}$ to allow recovery from preparation stress. Slices were then transferred into a submerged-type recording chamber and were allowed to adapt for at least $30 \mathrm{~min}$ before the experiment started. The chamber was constantly perfused with artificial cerebrospinal fluid (ACSF) at a rate of $2.5 \mathrm{ml} / \mathrm{min}$ at $33 \pm 1{ }^{\circ} \mathrm{C}$.

Synaptic responses were elicited by stimulation of the Schaffer collateral-commissural fibers in the stratum radiatum of the CA1 region using lacquer-coated stainless steel stimulating electrodes. Glass electrodes (filled with ACSF, 1-4 MS) were placed in the apical dendritic layer to record field excitatory postsynaptic potentials (FEPSPS). The initial slope of the FEPSP was used as a measure of this potential. The stimulus strength of the test pulses was adjusted to $30 \%$ of the FEPSP maximum. During baseline recording, single stimuli were applied every minute $(0.0166 \mathrm{~Hz})$. Once a stable baseline had been established, long-term potentiation was induced by applying 100 pulses at an interval of $10 \mathrm{~ms}$ and a width of the single pulses of $0.2 \mathrm{~ms}$ (strong tetanus) three times at 10 min intervals. ${ }^{15}$

\subsubsection{Measurement of Input-Output relation and Paired Pulse Ratio}

The input-output relationship was obtained from a curve of FEPSP amplitude vs. stimulation intensity. For paired pulse stimulation, the stimulus strength of the test pulses was adjusted to $50 \%$ of the fEPSP maximum. Paired-pulse stimulation was delivered with inter-stimulus interval of $20 \mathrm{~ms}$ and the pairedpulse ratio (PPR) was calculated as the ratio of the second fEPSP amplitude to the first. ${ }^{15}$

\footnotetext{
${ }^{15}$ Protocol description kindly provided by Raik Rönicke, DZNE Magdeburg
} 


\subsection{Computational Analyses}

Analysis of images taken by digital microscopy and western blot infrared imaging are described in 2.4.2.3.2 and 2.4.2.3.1, respectively. For DNA sequence organization and maintenance the ApE software (v2.0.45) was used.

\subsubsection{DNA Microarray}

Analysis of microarrays was performed as described previously (Agis-Balboa et al., 2011; Kerimoglu, 2012; Kerimoglu et al., 2013; Peleg et al., 2010). In summary, starting with scanned images, intensity data was extracted using Agilent Feature Extraction software, version 9.5.3.1, and analyzed using the Limma (Smyth et al., 2005) package of Bioconductor (Gentleman et al., 2004). In order to assure that the intensities had similar distributions across arrays, VSN normalization (Huber et al., 2002) was applied to the intensity values as a method for between-array normalization. To estimate the average group values for each gene and assess differential gene expression, a simple linear model was fit to the data, and group-value averages and standard deviations for each gene were obtained. To find genes with significant expression changes between groups, empirical Bayes statistics were applied to the data by moderating the standard errors of the estimated values (Smyth, 2004). Pvalues were inferred from the moderated t-statistic and corrected for multiple testing using the FDR method (Benjamini and Hochberg, 1995). Afterwards, the final output was filtered for probes showing a change in normalized intensity that was greater than $1.414-$ fold $\left(\log _{2}(\right.$ fold change $)=0.5$ ) with an adjusted $p$-value of $\operatorname{FDR}(p)<0.1$.

\subsubsection{RNA-seq}

For whole-transcriptome sequencing a streamlined, customized bioinformatics approach was used for state-of-the-art data analysis. Table 2-7 gives details on the steps involved in this pipeline and non-default parameter settings of the programs and scripts used. Tables 2-8 and 2-9 give basic parameters of RNA and data quality control, including RNA integrity number (RIN), library size and reference genome alignment rate. 
Table 2-7: RNA-seq analysis pipeline.

\begin{tabular}{|c|c|c|c|c|}
\hline Step & Description & Tool / Script & $\begin{array}{l}\text { Non-default } \\
\text { parameters }\end{array}$ & Citation \\
\hline \multicolumn{5}{|l|}{1} \\
\hline $1 \mathrm{a}$ & Demultiplexing & Illumina pipeline scripts & - & - \\
\hline $1 b$ & File conversion (.bcl $\rightarrow$.fastq) & Illumina pipeline scripts & - & - \\
\hline 2 & Quality control & FastQC & - & $\begin{array}{l}\text { - FastQC A Quality Control } \\
\text { tool for High Throughput } \\
\text { Sequence Data) }\end{array}$ \\
\hline 3 & $\begin{array}{l}\text { Mapping to reference genome } \\
\text { (GRCm38/mm10) }\end{array}$ & Bowtie2 (v2.0.7) & $\begin{array}{l}\text {--very-sensitive- } \\
\text { local }\end{array}$ & $\begin{array}{l}\text { (Langmead and Salzberg, } \\
\text { 2012) }\end{array}$ \\
\hline 4 & $\begin{array}{l}\text { File conversion and sorting } \\
\text { (.sam } \rightarrow \text {.sorted.bam) }\end{array}$ & SAMtools & - & (Li et al., 2009a) \\
\hline 5 & Read counting in transcripts & HTSeq (htseq-count) & $\begin{array}{l}\text {-m intersection } \\
\text { non-empty }\end{array}$ & $\begin{array}{l}\text { (HTSeq: Analysing high- } \\
\text { throughput sequencing } \\
\text { data with Python) }\end{array}$ \\
\hline 6 & $\begin{array}{l}\text { Detection of differential gene } \\
\text { expression }\end{array}$ & DESeq (R-package) & $\begin{array}{l}\text { (pairwise } \\
\text { comparisons of } \\
\text { groups) }\end{array}$ & (Anders and Huber, 2010) \\
\hline 7 & Visualization & SeqMonk & - & $\begin{array}{l}\text { (Babraham Bioinformatics } \\
\text { - SeqMonk Mapped } \\
\text { Sequence Analysis Tool) }\end{array}$ \\
\hline
\end{tabular}

Table 2-8: Sample properties and quality control for samples used for RNA-seq in the study of Kat2a function.

\begin{tabular}{|c|c|c|c|c|}
\hline Sample\# & Group & $\begin{array}{l}\text { RNA Integrity } \\
\text { Number (RIN) }\end{array}$ & $\begin{array}{l}\text { Unmapped Paired } \\
\text { Reads (Library Size) }\end{array}$ & $\begin{array}{c}\text { Bowtie2: Alignment } \\
\text { Rate [\%] }\end{array}$ \\
\hline 497 & $A$ & 9.0 & $2.28 \mathrm{E}+07$ & 98.7 \\
\hline 498 & A & 9.1 & $1.76 \mathrm{E}+07$ & 98.3 \\
\hline 507 & A & 8.8 & $3.25 \mathrm{E}+07$ & 98.8 \\
\hline 514 & A & 8.9 & $3.16 \mathrm{E}+07$ & 98.7 \\
\hline 515 & A & 8.9 & $2.45 \mathrm{E}+07$ & 98.9 \\
\hline 524 & A & 8.7 & $2.30 \mathrm{E}+07$ & 98.9 \\
\hline 487 & B & 9.1 & $2.99 \mathrm{E}+07$ & 98.9 \\
\hline 488 & B & 8.8 & $4.99 \mathrm{E}+07$ & 98.9 \\
\hline 496 & B & 9.0 & $2.52 \mathrm{E}+07$ & 98.8 \\
\hline 509 & B & 8.9 & $1.93 \mathrm{E}+07$ & 98.9 \\
\hline 531 & $B$ & 8.5 & $2.38 \mathrm{E}+07$ & 98.9 \\
\hline 505 & $C$ & 8.5 & $3.22 E+07$ & 98.8 \\
\hline 506 & $C$ & 8.9 & $3.09 \mathrm{E}+07$ & 98.7 \\
\hline 508 & C & 9.0 & $2.71 \mathrm{E}+07$ & 98.8 \\
\hline 525 & C & 8.9 & $2.83 \mathrm{E}+07$ & 98.9 \\
\hline 546 & $C$ & 8.8 & $3.07 \mathrm{E}+07$ & 99.0 \\
\hline 547 & C & 8.9 & $3.33 \mathrm{E}+07$ & 98.9 \\
\hline 478 & $D$ & 9.0 & $2.44 \mathrm{E}+07$ & 98.9 \\
\hline 479 & $D$ & 9.1 & $1.83 \mathrm{E}+07$ & 98.4 \\
\hline 480 & D & 9.1 & $2.20 \mathrm{E}+07$ & 98.5 \\
\hline 513 & $D$ & 8.9 & $2.89 \mathrm{E}+07$ & 98.9 \\
\hline 516 & D & 9.0 & $2.88 \mathrm{E}+07$ & 98.9 \\
\hline 517 & D & 8.9 & $2.49 \mathrm{E}+07$ & 98.9 \\
\hline 533 & D & 8.9 & $2.70 \mathrm{E}+07$ & 98.8 \\
\hline
\end{tabular}


Table 2-9: Sample properties and quality control for samples used for microarray and RNA-seq in the study of transcriptional changes during hippocampal aging.

\begin{tabular}{|c|c|c|c|c|}
\hline Sample\# & $\begin{array}{c}\text { Age } \\
\text { [months] }\end{array}$ & Assay & $\begin{array}{l}\text { Unmapped } \\
\text { Paired Reads } \\
\text { (Library Size) }\end{array}$ & $\begin{array}{c}\text { Bowtie2: } \\
\text { Alignment } \\
\text { Rate [\%] }\end{array}$ \\
\hline $3 m_{-} 1$ & 3 & Microarray & - & - \\
\hline $3 \mathrm{~m} \_2$ & 3 & Microarray & - & - \\
\hline $3 \mathrm{~m} \_3$ & 3 & Microarray & - & - \\
\hline $3 m \_4$ & 3 & Microarray & - & - \\
\hline$\geq 28 m_{-} 1$ & $\geq 28$ & Microarray & - & - \\
\hline$\geq 28 \mathrm{~m} \_2$ & $\geq 28$ & Microarray & - & - \\
\hline$\geq 28 \mathrm{~m} \_3$ & $\geq 28$ & Microarray & - & - \\
\hline$\geq 28 \mathrm{~m} \_4$ & $\geq 28$ & Microarray & - & - \\
\hline 3m_se_1 & 3 & RNA-seq SE & $2.89 E+07$ & 98.8 \\
\hline $3 \mathrm{~m} \_s e \_2$ & 3 & RNA-seq SE & $2.58 \mathrm{E}+07$ & 99.4 \\
\hline $3 \mathrm{~m} \_s e \_3$ & 3 & RNA-seq SE & $3.71 \mathrm{E}+07$ & 98.9 \\
\hline $3 m \_s e \_4$ & 3 & RNA-seq SE & $2.62 \mathrm{E}+07$ & 99.1 \\
\hline $3 \mathrm{~m} \_s e \_5$ & 3 & RNA-seq SE & $2.78 \mathrm{E}+07$ & 99.1 \\
\hline $24 m \_s e \_1$ & 24 & RNA-seq SE & $1.94 \mathrm{E}+07$ & 99.0 \\
\hline $24 m \_s e \_2$ & 24 & RNA-seq SE & $1.82 \mathrm{E}+07$ & 99.3 \\
\hline $24 m \_s e \_3$ & 24 & RNA-seq SE & $2.82 \mathrm{E}+07$ & 99.4 \\
\hline $24 m \_s e \_4$ & 24 & RNA-seq SE & $1.99 \mathrm{E}+07$ & 99.1 \\
\hline $24 \mathrm{~m}$ se 5 & 24 & RNA-seq SE & $2.41 \mathrm{E}+07$ & 99.2 \\
\hline $24 \mathrm{~m}$ _se_6 & 24 & RNA-seq SE & $1.24 \mathrm{E}+07$ & 99.4 \\
\hline $3 \mathrm{~m} \_\mathrm{PE} \_1$ & 3 & RNA-seq PE & $1.64 \mathrm{E}+07$ & 98.7 \\
\hline $3 m \_P E \_2$ & 3 & RNA-seq PE & $1.95 \mathrm{E}+07$ & 99.1 \\
\hline 3m_PE_3 & 3 & RNA-seq PE & $1.44 \mathrm{E}+07$ & 98.8 \\
\hline$\geq 28 \mathrm{~m}$ _PE_1 & $\geq 28$ & RNA-seq PE & $1.54 \mathrm{E}+07$ & 98.9 \\
\hline$\geq 28 m$ m_PE_2 & $\geq 28$ & RNA-seq PE & $1.60 \mathrm{E}+07$ & 98.1 \\
\hline$\geq 28 m$ m_PE_3 & $\geq 28$ & RNA-seq PE & $1.75 \mathrm{E}+07$ & 99.0 \\
\hline
\end{tabular}

\subsubsection{Downstream Analysis and Functional Annotation of Gene Expression Data}

Downstream analysis for genome-wide expression assessment methods comprised group comparisons (search for overlaps, calculate Venn diagrams) and functional annotation. To calculate Venn diagrams and retrieve lists with overlapping genes, the web tool of the BEG group, University of Gent was used. Functional annotation and category analysis was carried out using the Database for Annotation, Visualization and Integrated Discovery (DAVID, v6.7) (Huang et al., 2009a, 2009b).

\subsubsection{ChIP-seq}

DNA derived from ChIP (2.4.4) was sequenced as described in 2.4.2.1.5. Fastq-files were retrieved from Fasteris SA's FTP-server and steps 2, 3, 4 and 7 of the pipeline described in 2.6.2 were applied to the data and correlated with gene expression data from the corresponding RNA-seq experiments. 


\subsubsection{Statistical Analyses}

General-purpose statistical analysis for behavioral and molecular analyses was performed using GraphPad Prism 6 or Microsoft Excel for Mac 2008. Details of statistical analysis, methods and parameters are given in the results sections when data is described. Statistical tests include: Oneway/Two-way/repeated measures analysis of variance or repeated measures analysis of variance (1way/2way/rmANOVA), Tukey's multiple comparisons test (McHugh, 2011), Šidák's multiple testing correction (Sidak, 1967), the Benjamini-Hochberg procedure (FDR) (Benjamini and Hochberg, 1995), Student's t-test (Student, 1908). The number of biological replicates (n) is given the base of figures where necessary; figures belonging to the same experimental setup display these numbers only once to avoid redundancy. Statistical analysis of whole-genome data was performed as described in the respective sections $(2.6 .1,2.6 .2$, and 2.6.4). Statistical significance is indicated by asterisks $(*)$, hashes $(\#)$ on three different levels throughout graphs: $*: p<0.05, * *: p<0.01$, $* * *: p<0.001$.

Unless otherwise stated, error bars in all experimental figures represent standard error of the mean (SEM), which is calculated as

$$
S E M=\frac{S D}{\sqrt{n}}
$$




\section{Results}

The results section is separated in two main paragraphs with different focus each. While the first paragraph mainly covers the role of Kat2a in the adult murine brain and learning and memory, the second paragraph is dedicated to hippocampal aging and associated changes in transcriptional regulation.

For purposes of clarity, Table 3-1 shows a color guide that shall help to intuitively associate certain colors with experimental conditions.

Table 3-1: Color guide for the results section to help associate of figure colors with a certain condition.

Color guide

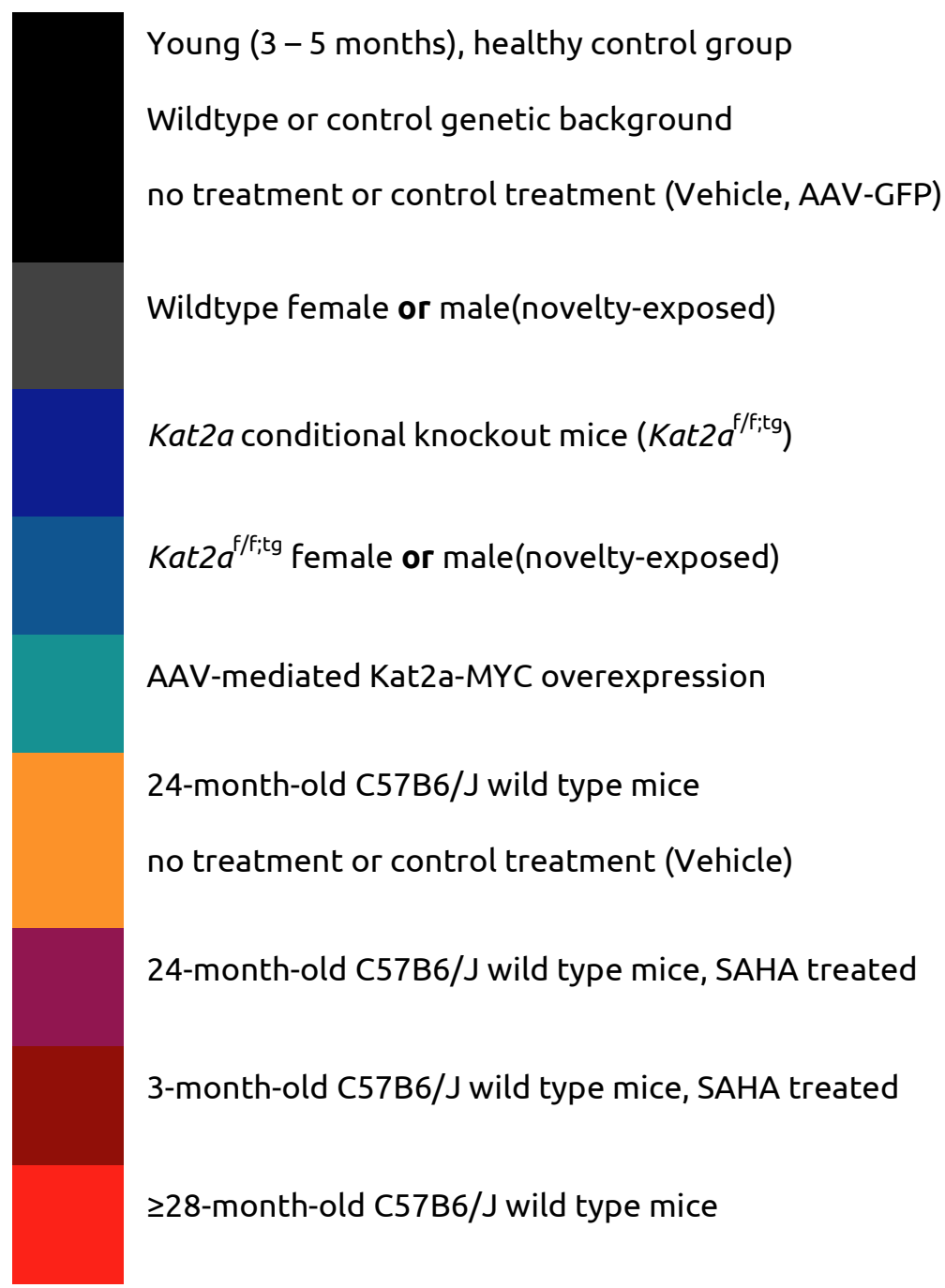




\subsection{The Role of Kat2a in the Adult Murine Brain and Learning and Memory}

\subsubsection{Characterization of a Kat2a Tissue-Specific Conditional Knockout Mouse}

Deficiency of Kat2a function has been shown to result in embryonic lethality due to failure of neural tube closure and exencephaly (Bu et al., 2007) and was found to exacerbate neurodegeneration when deleted in the context of spinocerebellar ataxia type 7 (SCA7) in the cerebellum (Chen et al., 2011). However, functional aspects of Kat2a in the adult forebrain and in the context of cognition have not yet been explored in detail. As several other HATs have been shown to be involved in such processes (see Stilling and Fischer, 2011 for review), we hypothesized that also Kat2a plays a role in learning and memory. This hypothesis is further supported by the observation that Kat2a is upregulated after contextual fear conditioning (Peleg et al., 2010) and is likely to acetylate H4K12, which is implicated in learning-induced gene expression (Bu et al., 2007; Ciurciu et al., 2008; Guelman et al., 2009; Hargreaves et al., 2009; Martinez et al., 2001; Peleg et al., 2010).

\subsubsection{Kat2a is Highly Expressed in CA1}

To explore the expression pattern of Kat2a within the adult murine brain we performed qRT-PCR and quantitative western blotting. In agreement with IN SITU hybridization data from the Allen Brain Atlas (Figure 6-1) (Lein et al., 2007), we observed high mRNA levels in the hippocampus as determined by qRT-PCR, with highest expression in the CA1 and CA3 region, and relatively low levels in cerebellar tissue. Two-way analysis of variance (2way ANOVA) showed a significant effect of genotype $\left(F_{(1,41)}=169.8, p<0.001\right)$ and brain region $\left(F_{(5,41)}=9.1, p<0.001\right)$. Tukey's multiple comparisons test revealed significant differences between brain regions as shown in Table 3-2. In the knockout condition, levels in all tested forebrain regions were significantly reduced to background levels ( 18\%). As the Cre-recombinase transgene is not expressed in the cerebellum (CB), mRNA abundance was similar to control mice (Figure 3-1A).

Table 3-2: mRNA levels - Tukey's multiple comparisons test after 2 way ANOVA. Only significant results are shown. Significant differences compared to cerebellum are displayed in Figure 3-1A.

\begin{tabular}{|c|c|c|c|c|c|c|c|c|}
\hline & Pfc vs. CA1 & Pfc vs. CA3 & DG vs. CA1 & DG vs. CB & CA1 vs. NC & CA1 vs. CB & CA3 vs. NC & CA3 vs. CB \\
\hline Mean Diff. & -1.448 & -0.9207 & -0.9575 & 0.7685 & 1.483 & 1.726 & 0.9558 & 1.198 \\
\hline Padj & $<0.001$ & 0.0011 & 0.0007 & 0.0093 & $<0.001$ & $<0.001$ & 0.0007 & $<0.001$ \\
\hline Significant? & $* * *$ & ** & $* * *$ & ** & $* * *$ & $* * *$ & $* * *$ & $* * *$ \\
\hline
\end{tabular}

We also determined Kat2a expression on the protein level, using nuclear lysates from the same brain regions and a Kat2a-specific antibody. One-way analysis of variance (1way ANOVA) revealed a significant difference between means. Tukey's multiple comparisons text showed significant differences between brain regions as shown in Table $3-3^{16}$.

\footnotetext{
${ }^{16}$ For this experiment, the total number of biological replicates was $n=3$. Data from 2 technical replicates of each biological replicate for CA1 and CB ( $n=3[6])$ was averaged before plotting and statistical analysis.
} 
Table 3-3: Protein levels - Tukey's multiple comparisons text after 1way ANOVA. Only significant results are shown. Significant differences compared to cerebellum are displayed in Figure 3-1B.

\begin{tabular}{|c|c|c|c|c|c|c|c|c|}
\hline & Pfc vs. DG & Pfc vs. CA1 & fc vs. CA & fc vs. CB & $A 1$ vs. $C A 3$ & CA1 vs. CB & CA3 vs. $\mathbf{N}$ & NC vs. CB \\
\hline Mean Diff. & 4.28 & 2.933 & 5.676 & 5.851 & 2.742 & 2.917 & -3.981 & 4.156 \\
\hline$P_{\text {adj }}$ & 0.0022 & 0.0336 & 0.0002 & 0.001 & 0.0498 & 0.0347 & 0.0039 & 0.0028 \\
\hline Significant? & $* *$ & * & $* * *$ & $* * *$ & * & * & $* *$ & $* *$ \\
\hline
\end{tabular}

Concordant with expression of Kat2a on the mRNA level, we found significantly higher levels of Kat2a protein in the CA1 region compared to cerebellum, where immunoreactivity was very weak. However, prefrontal cortical (Pfc) lysates as well as lysates from other parts of the neocortex (NC) showed higher levels compared to lysates from hippocampal regions (Figure 3-1B).

The Kat2a protein is highly enriched in nuclear fractions prepared form hippocampal tissue, with a faint signal observed in the cytosolic fraction. Effectiveness of the subcellular fractionation method is demonstrated by immunoreactivity of $\beta$-Actin (weaker band intensity in the nucleus) and Histone $\mathrm{H} 4$ (immunoreactivity only in nuclear fractions). Kat2a was drastically reduced in Kat2 $a^{\mathrm{f} / \mathrm{f} ; \mathrm{tg}}$ mice (Figure 3-1C). Quantification of protein levels in hippocampal nuclear lysates showed a decrease of $\sim 82 \%$, which is parallels the reduction of mRNA levels in CA1 (Figure 3-1A,D). We conclude that Kat2a is highly expressed in the murine CA1 subfield of the hippocampus and enriched in the nucleus, suggesting an important function in transcriptional regulation in this area. Expression levels of mRNA and protein are severely reduced specifically in forebrain regions of Kat2 $a^{\mathrm{f} / \mathrm{F} \text { tg }}$ mice, demonstrating functionality of the knockout approach. 

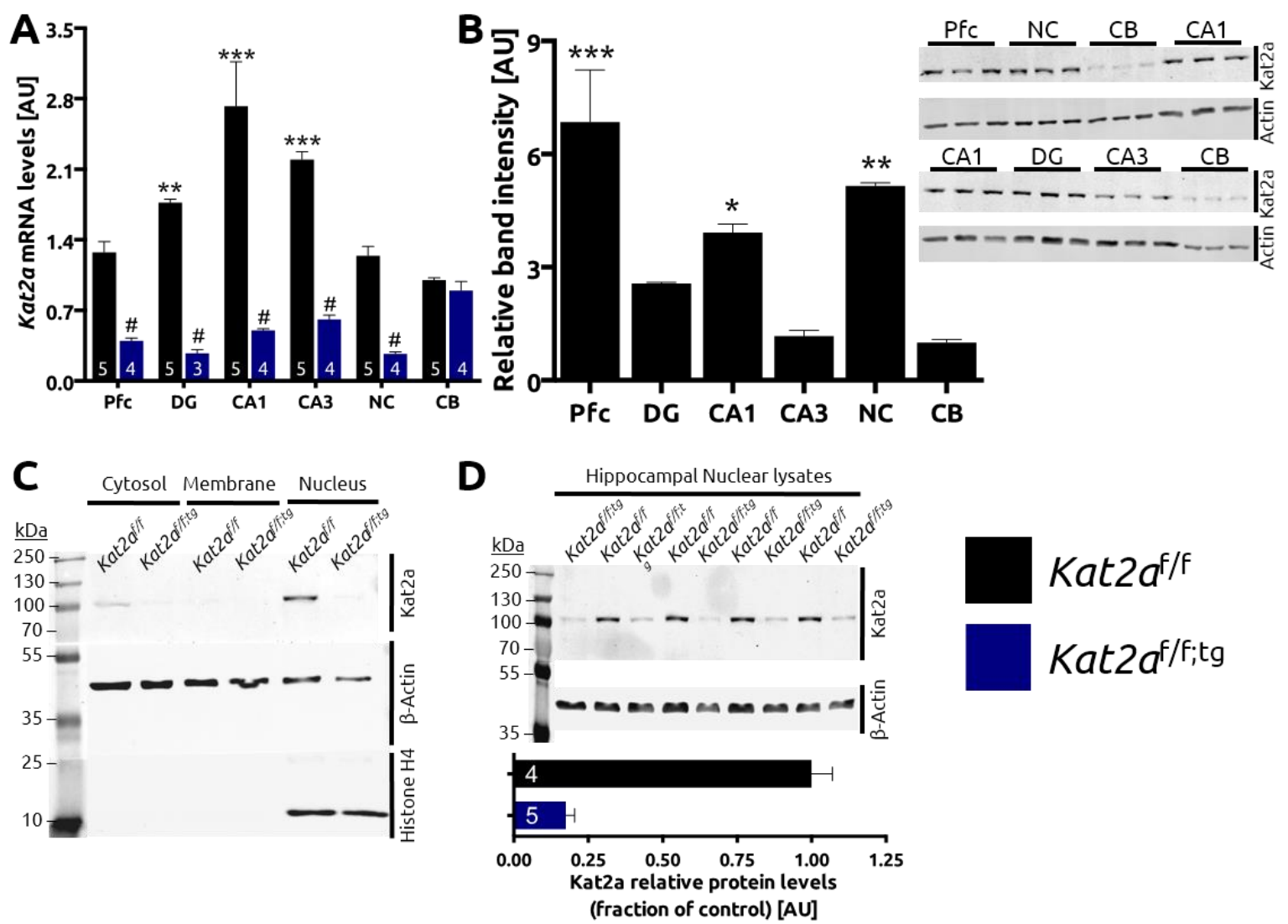

Figure 3-1: Nuclear localized Kat2a is differentially expressed in adult brain regions. qRT-PCR revealed that Kat2a mRNA levels are highest in the hippocampal region. Values are normalized to control cerebellar values. Asterisks represent a statistically significant difference in comparison to cerebellum; hashes represent a statistically significant difference between genotypes within brain regions (A). Kat2a protein levels within the hippocampus are highest in the CA1 region and lowest in the cerebellum. Cortical regions are also significantly enriched in Kat2a protein. Asterisks refer to significance with regard to cerebellum. $n=3$ for $P f c, D G, C A 3$ and NC; $\mathrm{n}=3(6)$ for $C A 1$ and $C B(B)^{10}$. Hippocampal Kat2a protein is enriched in the nucleus, compared to cytosolic and membrane fractions (C) and reduced by $80 \%$ in Kat2 $a^{\mathrm{f} / f ; t g}$ mice (D). $A U$, arbitrary units.

\subsubsection{Kat2a Conditional Knockout Mice Show no Overt Morphological Alterations}

We measured body and brain weight of adult mice to determine potential developmental effects in Kat2a conditional knockout (cKO) mice. Average body weight was slightly reduced in male Kat2 $a^{\mathrm{F} / \text {; }: \mathrm{tg}}$ mice of ages 3 to 5 months as well as 5-8 months (Figure 3-2A). This observation was underlined by a reduced body size as shown in the representative picture in Figure 3-2C. Female $K a t 2 a^{\mathrm{F} / \text {;:tg }}$ mice only showed a trend towards reduced body weight (Figure 3-2). Interestingly, both male and female Kat2a/fitg mice had significantly reduced brain weights (Figure 3-2B). However we could not detect gross morphological differences in by inspection of isolated brains as seen in the representative picture Figure 3-2D. Furthermore, the relation of brain weight and body weight did not show any difference between genotypes (Figure 3-2E). 
A

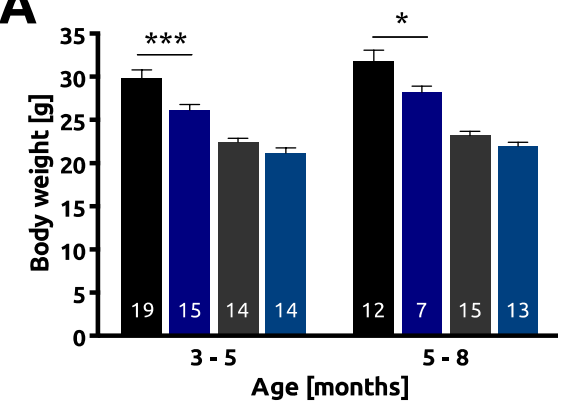

C

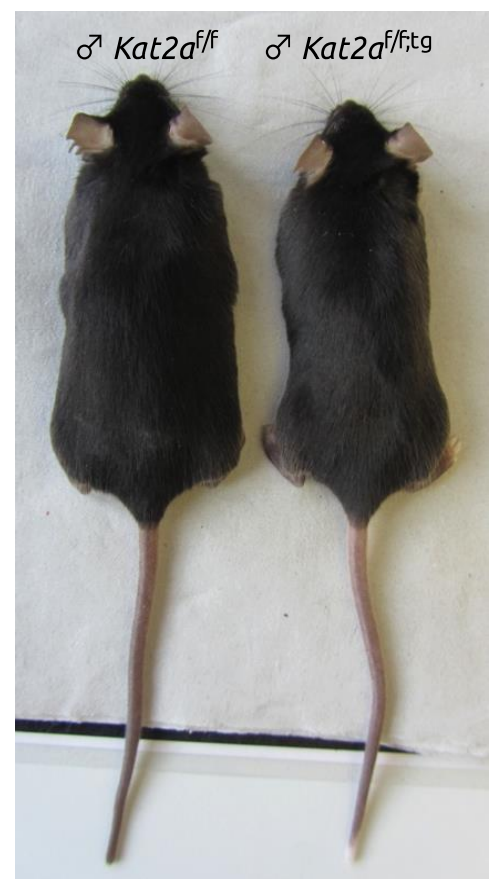

B

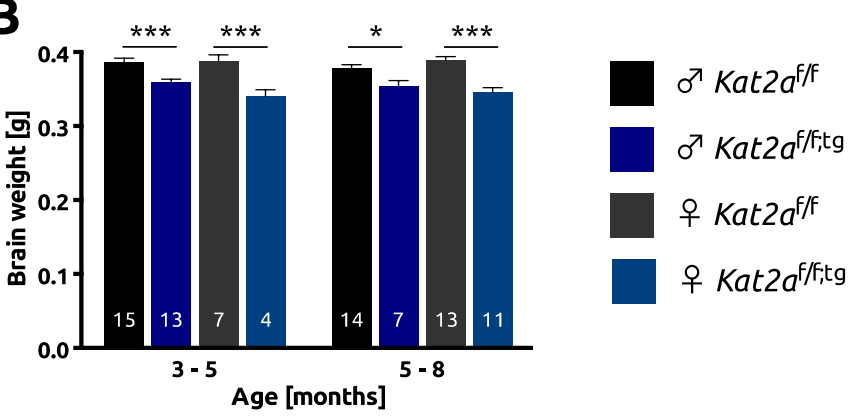

D

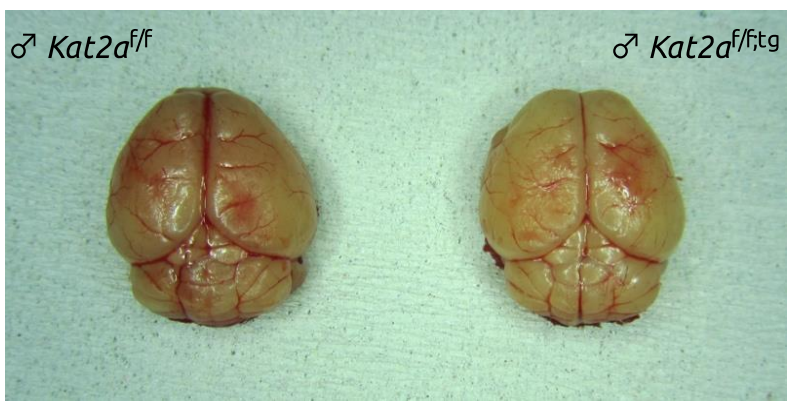

$\mathbf{E}$

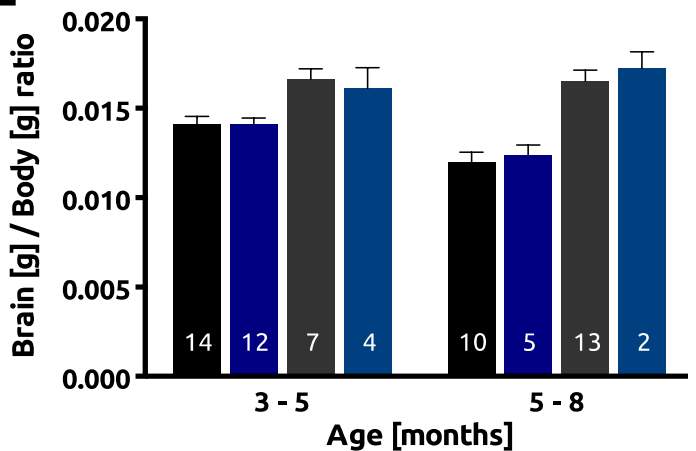

Figure 3-2: Anatomical alterations in $K a t 2 a^{\mathrm{f} / F ; t g}$ mice. Male but not female cKO mice show reduced body weight in two age groups and reduced body size (A). Both male and female mice have reduced brain mass, but no overt differences in brain morphology, as shown in representative images (B). Representative image of 3month-old siblings (C) and their brains (olfactory bulbs removed, D). The ratio of brain weight to body weight was independent of genotype (E).

To further investigate potential morphological alterations in the hippocampus of Kat2a cKO mice in more detail, we performed DNA staining and immunohistological stainings for neuronal and preand post-synaptic marker proteins, as well as for Kat2a itself (Figure 3-3). Despite strong background immunoreactivity, Kat2a staining vas visible in neuronal nuclei within the hippocampal subregions CA1, CA3 and DG. This signal was absent in Kat2 $a^{\mathrm{f} / \text {;itg }}$ mice. (Figure 3-3A). DAPI PANnuclear and neuronal nuclei NeuN (Rbfox3) staining did not reveal any obvious variations in cell distribution or morphology (Figure 3-3B,C). Post-synaptic (Map2) and pre-synaptic (Synaptophysin, Syp and Synaptoporin, Synpr) markers showed similar distributions and intensities (Figure 3-3C,D). 
A

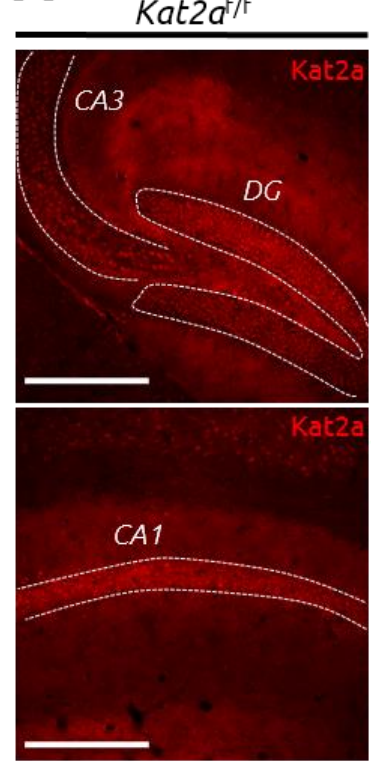

C
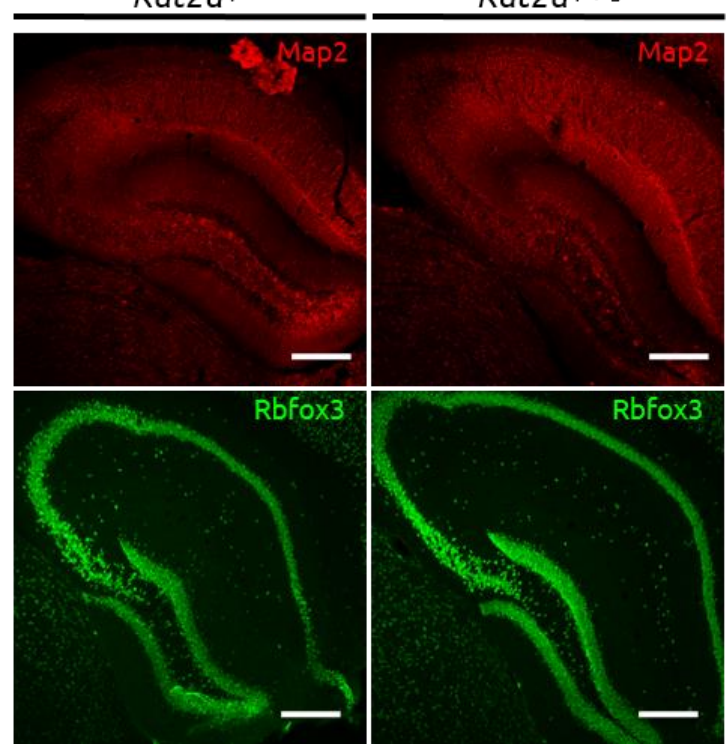

B
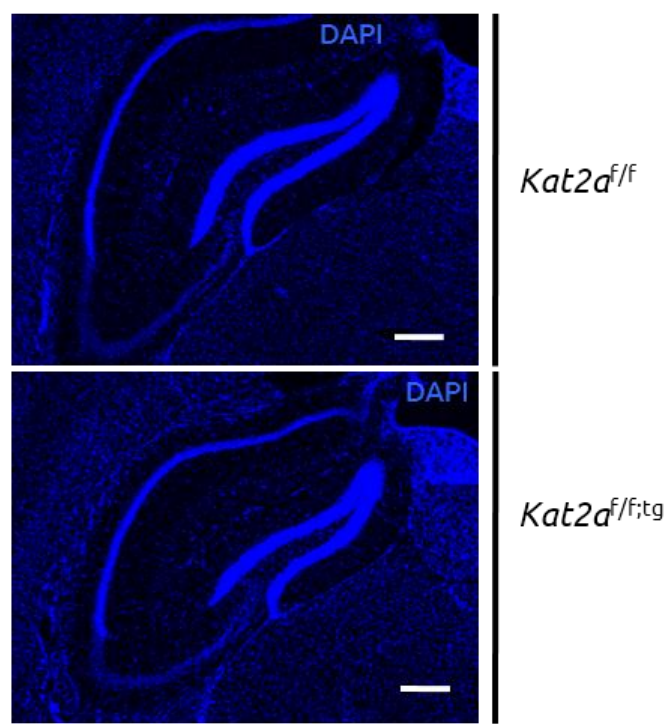

D

$\mathrm{Kat} 2 \mathrm{a}^{\mathrm{f} / \mathrm{f}}$

$\mathrm{Kat} 2 \mathrm{a}^{\mathrm{f} / \mathrm{f} ; \mathrm{tg}}$

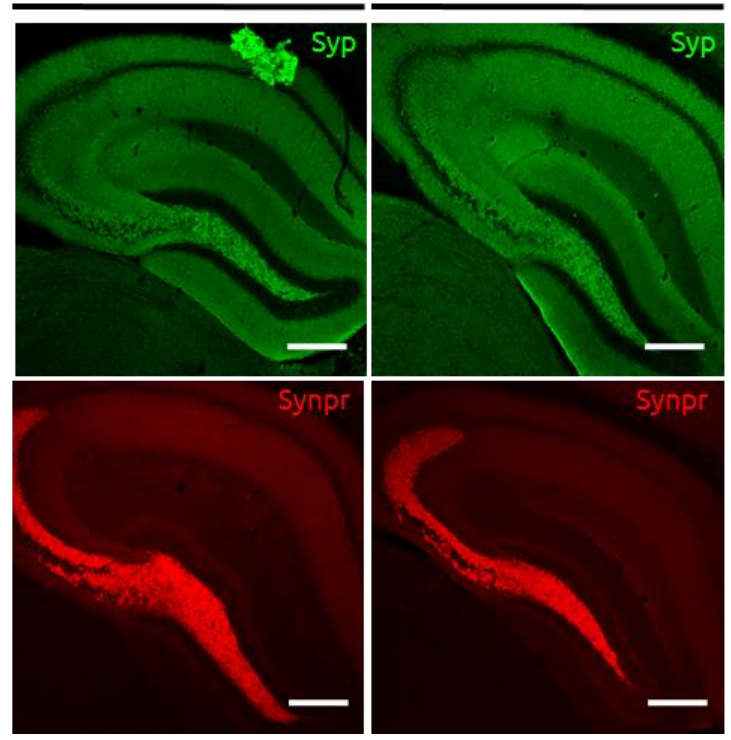

Figure 3-3: Morphological structure of the hippocampus of Kat2a cKO and control mice. Staining for Kat2a shows loss of Kat2a protein in hippocampal neuronal layers (A). DAPI is a PAN-nuclear DNA stain while NeuN (Rbfox3) is a marker neuronal nuclei (B,C). Map2 is localized to the post-synapse and dendrites while Synaptophysin (Syn) marks post-synaptic terminals (C,D). Synaptoporin (also known as Synaptophysin2) is a marker for mossy-fiber-specific pre-synaptic terminals (D). Representative images, scale bar: $250 \mu m$ in $\mathbf{A}, \mathbf{C}, \mathbf{D}$ and $500 \mu \mathrm{m}$ in $\mathbf{B}$. 
Glia, such as astrocytes and oligodendrocytes, are non-neuronal cells that constitute a large percentage of the mammalian brain (Bandeira et al., 2009). Since they do not activate the CamKIla promoter (Vazdarjanova et al., 2006), glial cells carry a functional Kat2a allele. However, gila cells have important supporting functions within nervous tissue such as nutritional supply, insulation and disposal of degenerated neurons (Schütz, 2008). Thus, they may respond to potential changes in adjacent, mutant neurons. We therefore stained cryosections from young adult Kat2 $a^{f / f ; t g}$ and control mice for the glial marker proteins Gfap and Plp1 to visualize abundance and distribution of astrocytes and oligodendrocytes in the hippocampus, respectively. We did, however, not observe alterations in abundance or distribution of these cells (Figure 3-4A,B).
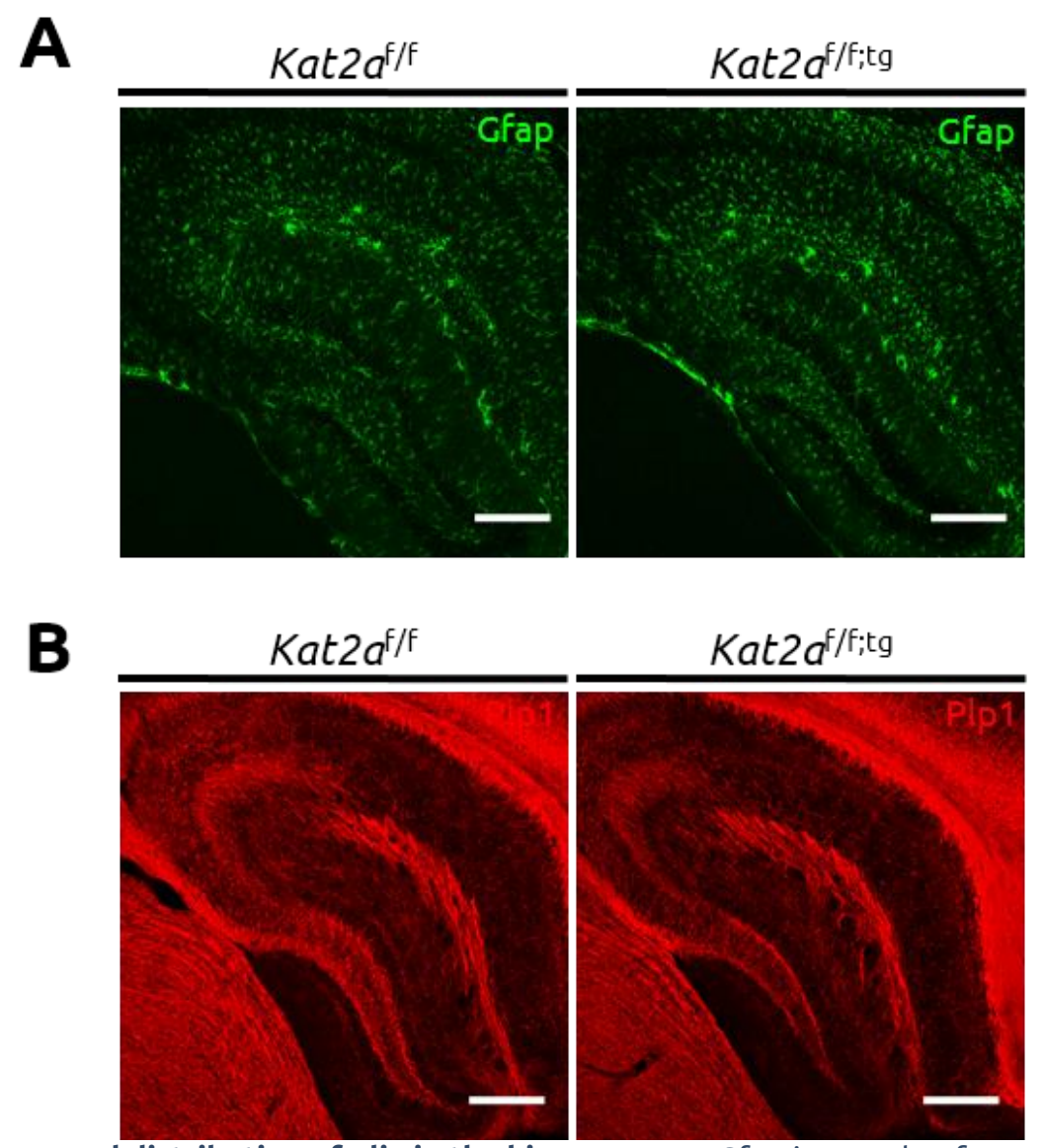

Figure 3-4: Abundance and distribution of glia in the hippocampus. Gfap is a marker for astrocytes (A) while

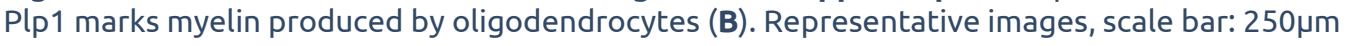

In conclusion, anatomical changes in Kat2 $a^{\mathrm{f} / \text {; tg }}$ mice with regard to overall body size were not reflected on the morphological level in the hippocampus. Furthermore, abundance and distribution of astrocytes and oligodendrocytes did not yield any evidence for potential deterioration of nervous tissue in the hippocampus. 


\subsubsection{Kat2a Conditional Knockout Mice Show Impairments in Hippocampus-Dependent Long-Term} Memory

Next, we analyzed potential behavioral effects of Kat2a loss-of-function by subjecting groups of male and female $K a t 2 a^{f / f i t g}$ mice to a behavioral test battery. Starting with an open field test to monitor exploratory behavior and locomotion, we found both groups spending comparable amounts of time in the center region of the arena and traveling similar distances within 5 min. Hence, there was also no significant difference in average traveling speed (Figure 3-5A).

Locomotion, coordination, motor-learning and physical strength was tested using the Rotarod performance test (Figure 3-5B). rmANOVA revealed a significant effect of time $\left(F_{(3,57)}=3.54, p=0.02\right)$ which could be attributed to the comparison of day 1 vs. day 4 (Tukey's multiple comparisons test: $p=0.029)$. However, there was no effect of genotype.

In the elevated plus maze anxiety and exploratory behavior was tested. Both groups showed normal levels of anxiety, as they spent most of the time ( $85-95 \%)$ in the closed arms or the center region. There was no difference in time spent in the open arms of the maze (Figure 3-5C).

Working memory was probed by monitoring the sequence of arm entries in a 4-arms cross maze, Successful alterations were defined as visits of an arm that was not visited within the last three trials before. Both groups performed significantly better than the $9.375 \%$-chance level, which indicates non-random arm entries. (Student's one-sample t-test: Kat2 $a^{f / F}: p<0.001, K a t 2 a^{f / f ; t g}$ : $p=0.001$ ). However, there was no significant difference between groups (Figure 3-5D)

A novel object recognition (NOR) paradigm was used to determine potential defects in short-term as well as long-term recognition memory. After habituation, both groups showed no preference for one of the two equal objects during the training session (Figure 3-5E, TR) and equivalent preference for the novel object in the short-term memory paradigm (Student's one-sample t-test vs. 0.5: $\mathrm{p}<0.001$ for both groups; Figure 3-5E, STM). Also in the long-term paradigm, both groups showed significant preference for the novel object (Student's one-sample t-test vs. 0.5 : Kat2 $a^{\mathrm{f} / \mathrm{f}}: p<0.001$, Kat2 $\left.a^{f / f ; t}: p=0.0017\right)$. Interestingly however, Kat2 $a^{\mathrm{f} / f ; t g}$ mice displayed a slightly reduced object preference index when compared with the control group (Student's t-test: $p=0.041$; Figure 3-5E, LTM).

We next tested associative learning in a contextual fear-conditioning (cFC) paradigm that is known to be hippocampus dependent (Anagnostaras et al., 2001). While baseline freezing in the training situation was comparable, Kat2 $a^{\mathrm{f} / \text {;itg }}$ mice showed significantly less freezing behavior when reintroduced to the context 24 hours after receiving the electric foot shock (Student's t-test: $P_{a d j}=0.015$; Figure 3-5F, right panel). To exclude effects of altered sensory perception in Kat $2 a^{f / f ; t g}$ mice, we also monitored shock response activity during the 2s-shock phase (Figure 3-5F, right panel). 
A
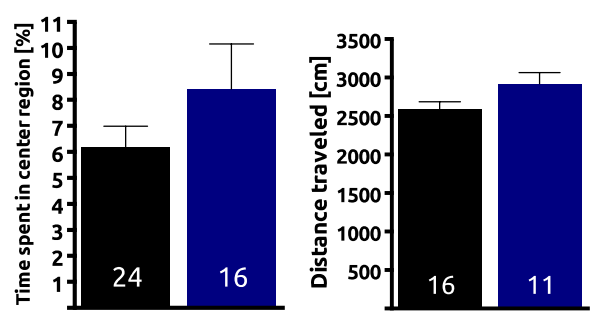

C

Elevated Plus Maze

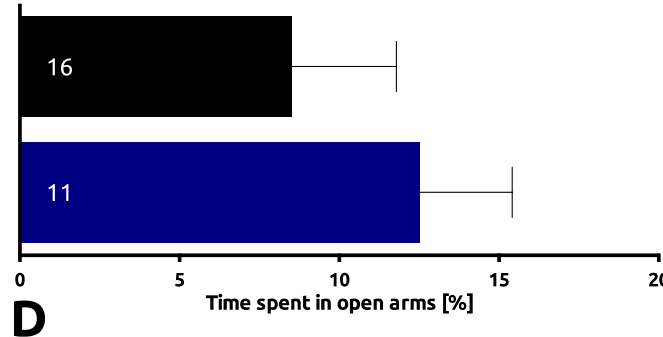

$5 \quad$ Time spent in op
Cross Maze

9,4\%:chance level

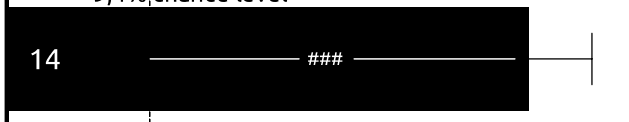

\section{0}

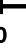

F

E

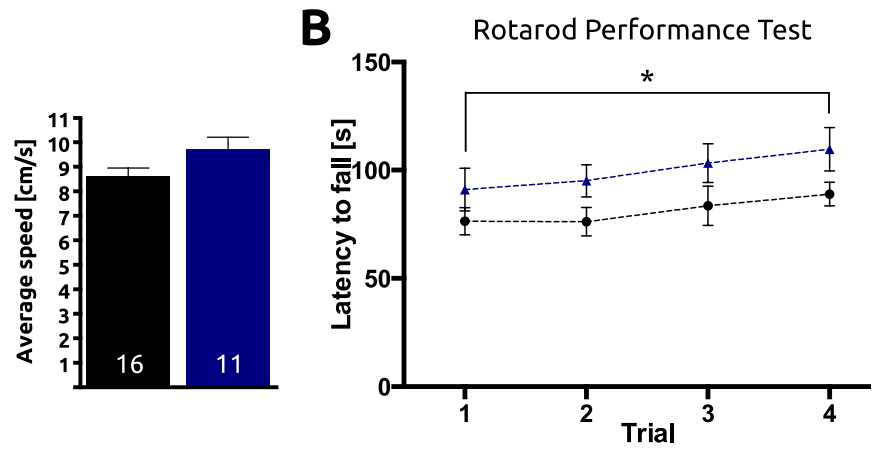

E
Novel Object Recognition
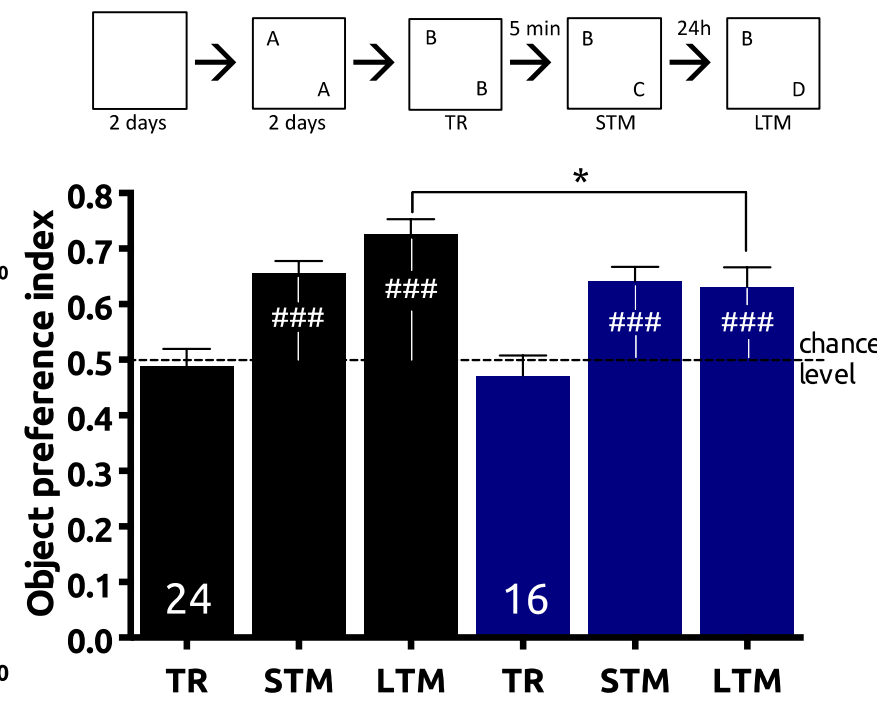

Contextual Fear Conditioning
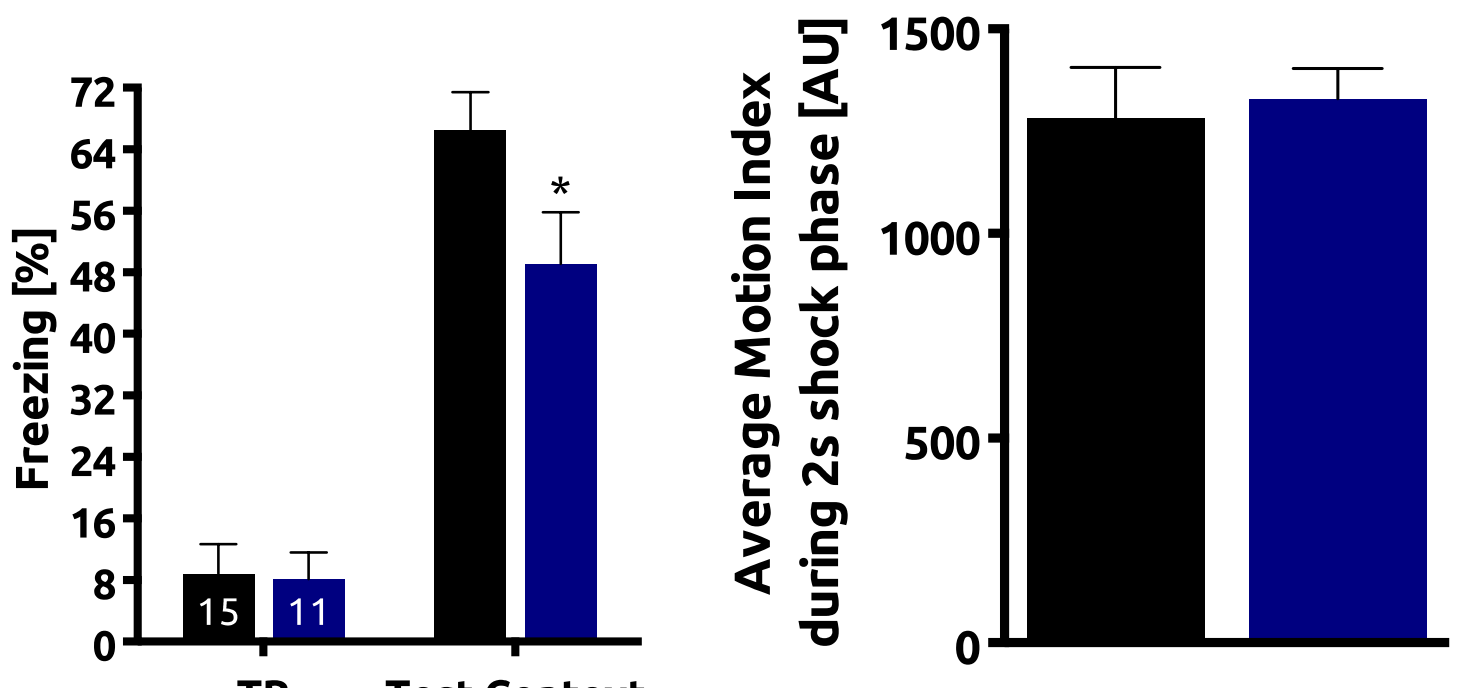

TR Test Context

- $\mathrm{Kat2a}^{\mathrm{f} / \mathrm{F}}$

$\operatorname{Kat} 2 a^{\mathrm{f} / \mathrm{F}, \mathrm{tg}}$

Figure 3-5: Behavioral analysis of Kat2a loss-of-function. Open field test (A), Rotarod performance test (B), Elevated plus maze (C) and 4-arms cross maze (D) indicated normal behavior with regard to locomotion, exploratory behavior, basal anxiety and working memory. Novel object recognition (E) and contextual fear conditioning (F) revealed a tendency to impaired long-term memory. Control parameters like object preference during training (E, TR) or activity in response to electric foot shock (F, right panel) were comparable. Asterisks indicate significant differences between groups. Hashes indicate significant differences from chance level within groups. TR, Training; STM, Short-term memory; LTM, Long-term memory. 
Finally, we subjected mice to a spatial navigation task known as Morris watermaze (MWM). To exclude a potential effect of impaired vision, we first installed a visible platform in the pool. Though both groups rapidly learned to navigate within the pool and find the platform, Kat $2 a^{f / f ; t g}$ mice showed a slight delay during day 2 (rmANOVA, main effect of time: $F_{(3,54)}=78.6, p<0.001$; main effect of genotype: $F_{(1,18)}=4.7, p=0.044$, Šidák's multiple comparisons test: Day2, $p=0.015$; Figure 3-6A, left panel).

A different group of mice was used to test memory using a hidden-platform task. As shown in the right panel of Figure 3-6A, Kat2 $a^{\mathrm{f} / \mathrm{fitg}}$ mice displayed a significant retardation in finding the hidden platform (rmANOVA, main effect of time: $F_{(8,176)}=21.6, p<0.001$; main effect of genotype: $F(1,22)=18.04, p<0.001$; Šidák's multiple comparisons test: Day2, $p=0.004$; Day3, $p=0.006$; Day5, $p<0.001$; Day7, $p=0.011$; Day9, $p=0.02$ ). To exclude effects of potential mobility impairments to account for this difference, we also monitored the swim speed on day 1 of the test, where potential motivational differences are most likely negligible (Figure 3-6B).

At the day of the probe test, the platform was removed and time spent in the four quadrants of the pool was monitored for one minute. Time spent in the target quadrant (TQ) was significantly different from chance level (Student's one-sample $t$-test: $p=0.013$ ) as well as from average time spent in the other three quadrants (Q1-3, Student's t-test: $\mathrm{p}=0.015)$ in Kat2 $a^{\mathrm{f} / \mathrm{f}}$ mice (Figure 3-6C). However, Kat2 $a^{f / f i t g}$ mice did not show preference for the target quadrant. Also the number and integrated time of crossing over the former platform region was reduced in Kat $2 a^{\mathrm{f} / \mathrm{f}}$ mice, though these differences were not statistically significant (Student's t-test: Platform crossings: $p=0.096$, Time: $p=0.278$ ).

In conclusion, Kat2a loss-of-function did neither result in changed locomotion or baseline anxiety levels nor was there any evidence for altered working memory or short-term recognition memory. Indeed, memory impairments were only found in hippocampus-dependent forms of long-term memory, like contextual aversive memory and spatial memory as demonstrated by CFC and MWM, respectively, accompanied by a slight reduction in long-term NOR memory strength. 
A

Morris Water Maze

B

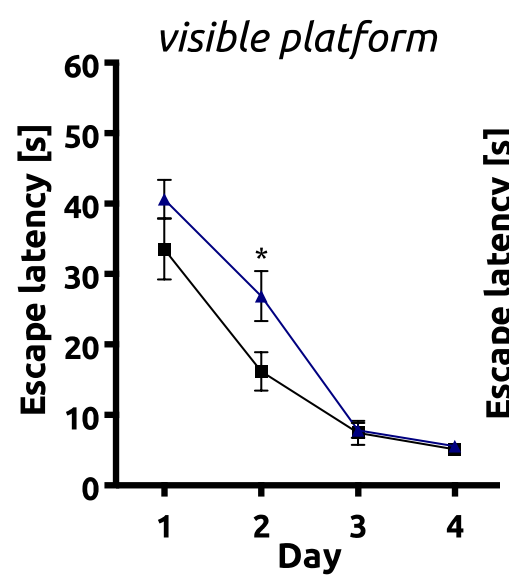

C
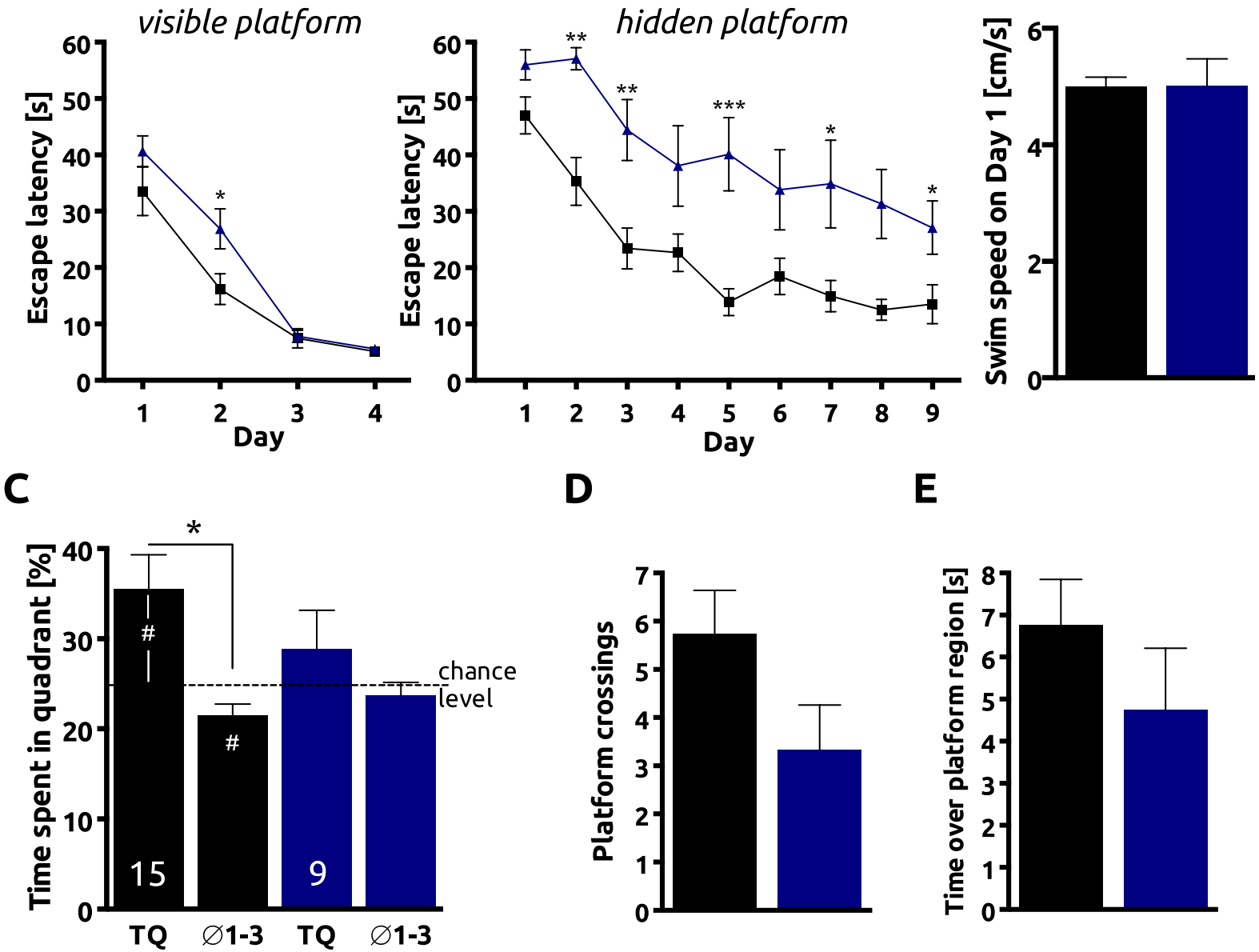

D

E
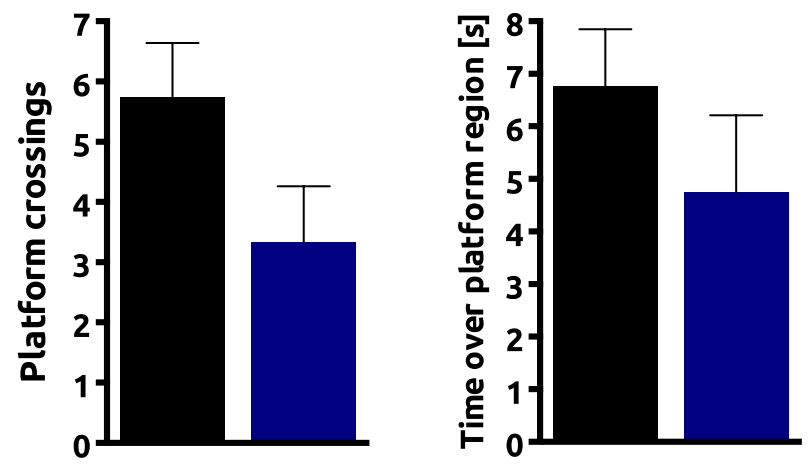

Kat2a $a^{f / f}$

$K a t 2 a^{f / f, t g}$

Figure 3-6: Morris watermaze. Learning curves in a visual and a memory-dependent form of the task (A). Swim speed on day 1 of the test (B). Time spent in each of the four quadrants on the day of the probe test (C). Number of platform crossings and summed time spent over former platform region during probe test $(\mathbf{D}, \mathbf{E})$. Asterisks indicate significant differences between groups. Hashes indicate significant differences from chance level within groups. TQ, Target quadrant; 1-3, quadrants 1 to 3. 


\subsubsection{Kat2a Conditional Knockout Mice Show Impaired Long-Term Potentiation in the CA1}

In order to explore a potential neurophysiological basis for the observed behavioral phenotype in Kat2a cKO mice, we next investigated electrical properties of CA3-to-CA1 communication in acute hippocampal slices ${ }^{17}$. To this end, CA3-originating projections (Schaffer collateralcommissural fibers) were externally stimulated and electric field potentials (excitatory postsynaptic potentials, fEPSPs) were recorded in the apical dendritic layer of the CA1 of Kat2 $a^{\mathrm{f} / \text {; titg }}$ and control mice as diagramed in Figure 3-7A). In both groups stimulation strength was well correlated with the elicited fEPSP slope, yielding similar linear input / output curves (Figure 3-7B).

Baseline fEPSP slopes were similar in both groups during the first $45 \mathrm{~min}$ of the measurement. Moreover, both groups showed a strong increase in fEPSP slope in response to three strong tetanic stimulations (LTP). Afterwards fEPSP slopes decayed slowly over time. Interestingly however, the change in FEPSP slope in Kat2 $a^{f / f i t g}$ mice did not reach the same level as compared to controls and showed a more rapid decay ( $r \mathrm{mANOVA}$, main effect of time: $F_{(285,2280)}=116.9, p<0.001$, main effect of genotype: $F_{(1,8)}=8.3, p=0.021$, interaction: $\left.F_{(285,2280)}=3.3, p<0.001\right)^{18}$. While control fEPSP slopes remained high over the whole time of measurement, fEPSP slopes of Kat2 $a^{\mathrm{f} / f \mathrm{i} t \mathrm{fg}}$ mice returned to baseline within this time (Figure 3-7C). In order to study short-term synaptic plasticity, a paired pulse stimulation paradigm was used. Here, we could not detect any difference between groups (Figure 3-7D).

In summary, while basic postsynaptic electrical properties of CA1 pyramidal and short-term synaptic plasticity were not different in the cKO condition, Kat2 $a^{f / f i t g}$ mice showed LTP impairments with lower LTP amplitude and faster decay of the potentiation effect.

\footnotetext{
17 These experiments were carried out at the DZNE-Magdeburg, in close collaboration with Prof. Dr. Klaus Reymann, Dr. Raik Rönicke and Katrin Böhm.

${ }^{18}$ For these experiments the total number of mice was $n=5$ for both groups resulting in $n=17$ and $n=20$ replicate measurements (sections) for $K a t 2 a^{f / f ; t g}$ and $K a t 2 a^{f / f}$ mice, respectively. Measurements for each mouse were averaged before plotting and statistical analysis.
} 

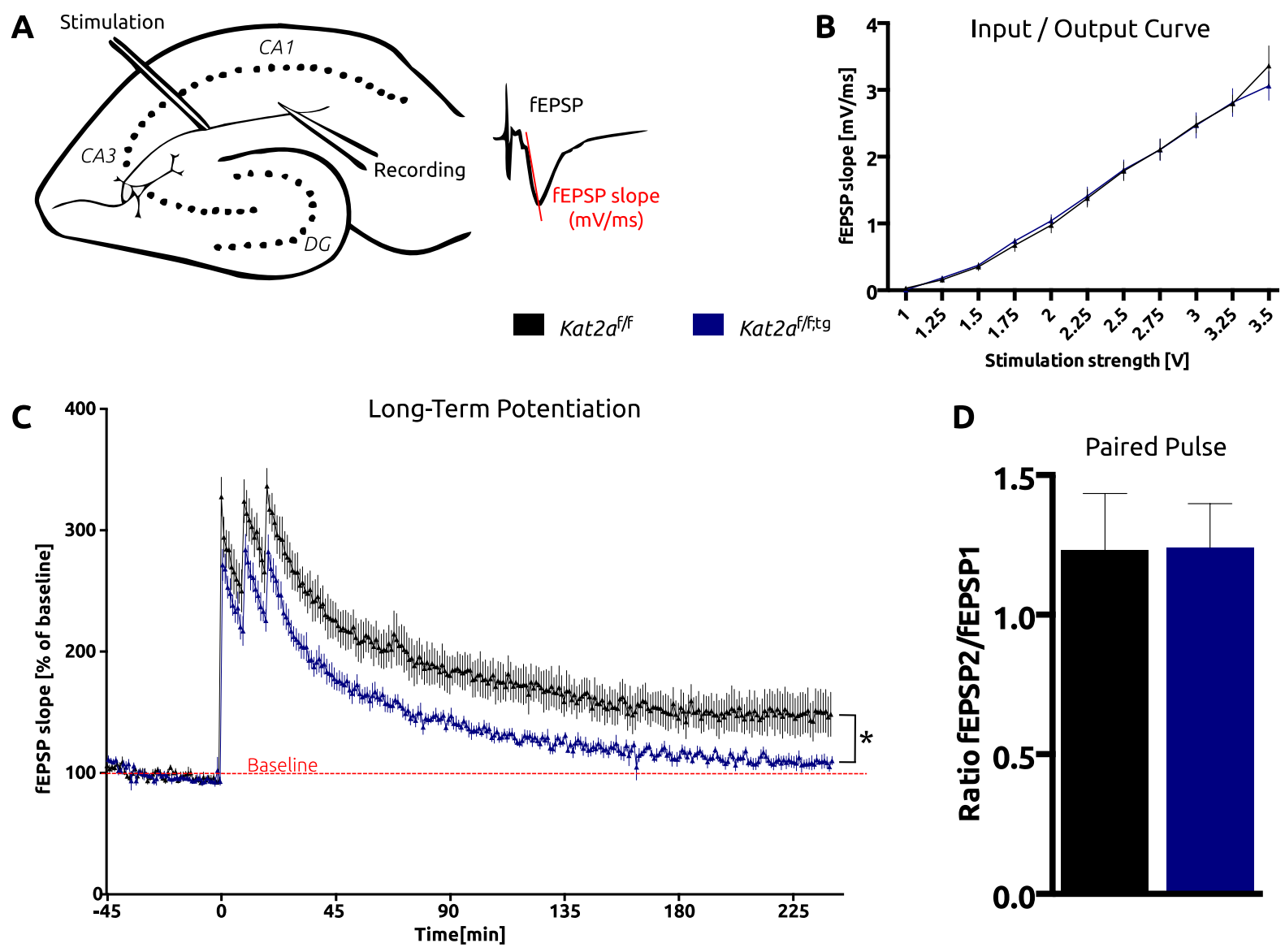

Figure 3-7: Electrophysiological analysis of long-term potentiation and paired pulse facilitation. Schematic overview of experimental setup (A). Input / Output curve, showing linear correlation of stimulations strength and fEPSP slope (B). LTP measurement over 240 minutes, starting with three strong tetanic stimuli after 45 minutes of baseline measurement (C). A test for Paired pulse facilitation or inhibition showed no

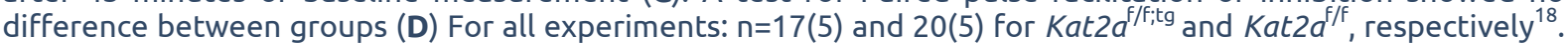
The asterisk in $\mathbf{C}$ represents significant difference with regard to the main effect of genotype. 


\subsubsection{Kat2a Conditional Knockout Mice Show Specific Alterations in Naïve and Stimulus-Dependent} Gene Expression in CA1

To analyze transcriptional changes resulting from Kat2a deletion in naïve mice as well as in a stimulus-dependent manner, we subjected $K a t 2 a^{\mathrm{F} / F \text { tg }}$ and control mice to a four-object noveltyexposure paradigm as described in 2.3.8. A graphical overview is given in Figure 3-8.

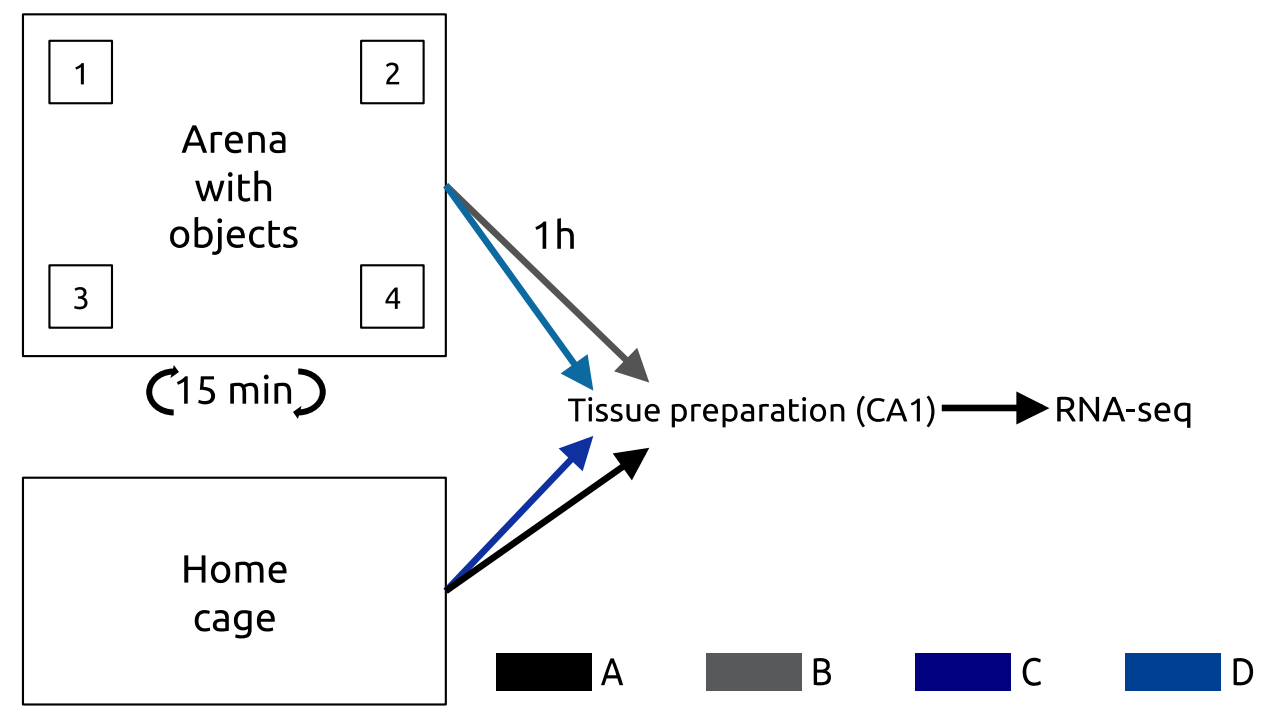

Figure 3-8: Schematic overview of novelty-exposure protocol for stimulus-dependent expression analysis. Mice of both genotypes were either exposed to a novel environment with 4 different objects (1-4) for 15 min and afterwards sent back to their home cage for 1 hour or directly taken from home cage. CA1 tissue was extracted from all mice, resulting in 4 different groups (A-D) used for RNA-seq to determine transcriptional changes.

For clarity purposes, the four resulting groups were named $\boldsymbol{A}$ to $\boldsymbol{D}$ as shown in Figure 3-8 and described in Figure 3-9A: A represents naïve $K a t 2 a^{\mathrm{f} / \mathrm{F}}$ mice (naïve control group), $\mathbf{C}$ are their Kat2adeficient counterparts (naïve Kat2 $a^{\mathrm{f} / \text {; }: \mathrm{tg}}$ ), $\mathbf{B}$ and $\mathbf{D}$ are the groups of Kat2 $a^{\mathrm{f} / \mathrm{F}}$ and $K a t 2 a^{\mathrm{f} / \mathrm{F} \text { tg }}$ mice, respectively, that underwent novelty exposure.

RNA-sequencing (RNA-seq) yielded 18-50 million paired reads of $200 \mathrm{bp}(100+100 \mathrm{bp}$ ) per sample with comparable distribution of total read count (Figure 3-9B). Reads were of high quality, with an average Phred quality score of $>30$ across the full read length (Figure 3-9C). Alignment of reads to the mouse genome using Bowtie2 resulted high alignment rates in all samples (see Table 2-8).

Interestingly, 2-dimensional principal component analysis (PCA) revealed that "novelty exposure" (likely component 2) seemed to explain more variance than "genotype" did, as shown by a clear separation of clusters $B$ and $D$ from clusters $A$ and $C$ in the $2^{\text {nd }}$ dimension of the PCA plot. Clustering of individual groups was more ambiguous (Figure 3-9D). Expression-based sample distances confirmed this notion, as not all samples from the same group did cluster together in a sample distance plot. Still, several samples within each group showed good correlation, e.g. within group $D$ (Figure 3-9E).

A great advantage of modern paired-end RNA-seq technology is the possibility of visualization of aligned reads and therefore transcripts. Figure 3-10F shows reads aligned at the Kat2a genomic locus. The aligned pairs are not split in this view so that they are visualized by a line (red or blue) corresponding to the length of the paired reads plus their distance. As the reads originate from 


\begin{tabular}{|c|c|c|}
\hline \multicolumn{2}{|c|}{ Condition } & \multirow{2}{*}{$\begin{array}{l}\text { Group } \\
\text { Name }\end{array}$} \\
\hline Genotype & Treatment & \\
\hline Kat2 $d^{f / f}$ & Naïve & A \\
\hline Kat2 $d^{d / f}$ & $\begin{array}{l}\text { Novelty } \\
\text { exposed }\end{array}$ & B \\
\hline Kat2a $a^{f / f ; t g}$ & Naïve & C \\
\hline Kat2 $a^{f / f, t g}$ & $\begin{array}{l}\text { Novelty } \\
\text { exposed }\end{array}$ & D \\
\hline
\end{tabular}
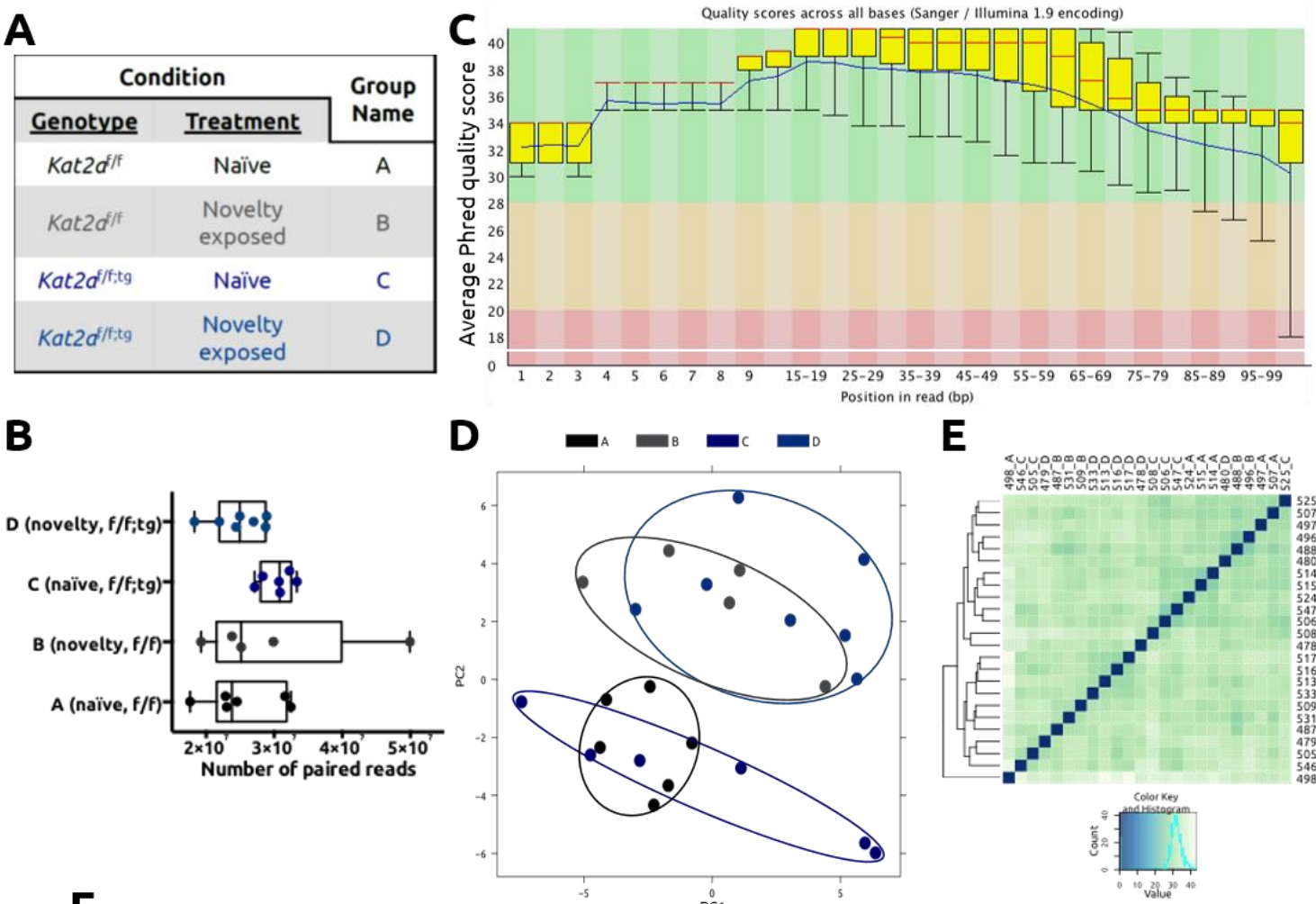

\section{E}
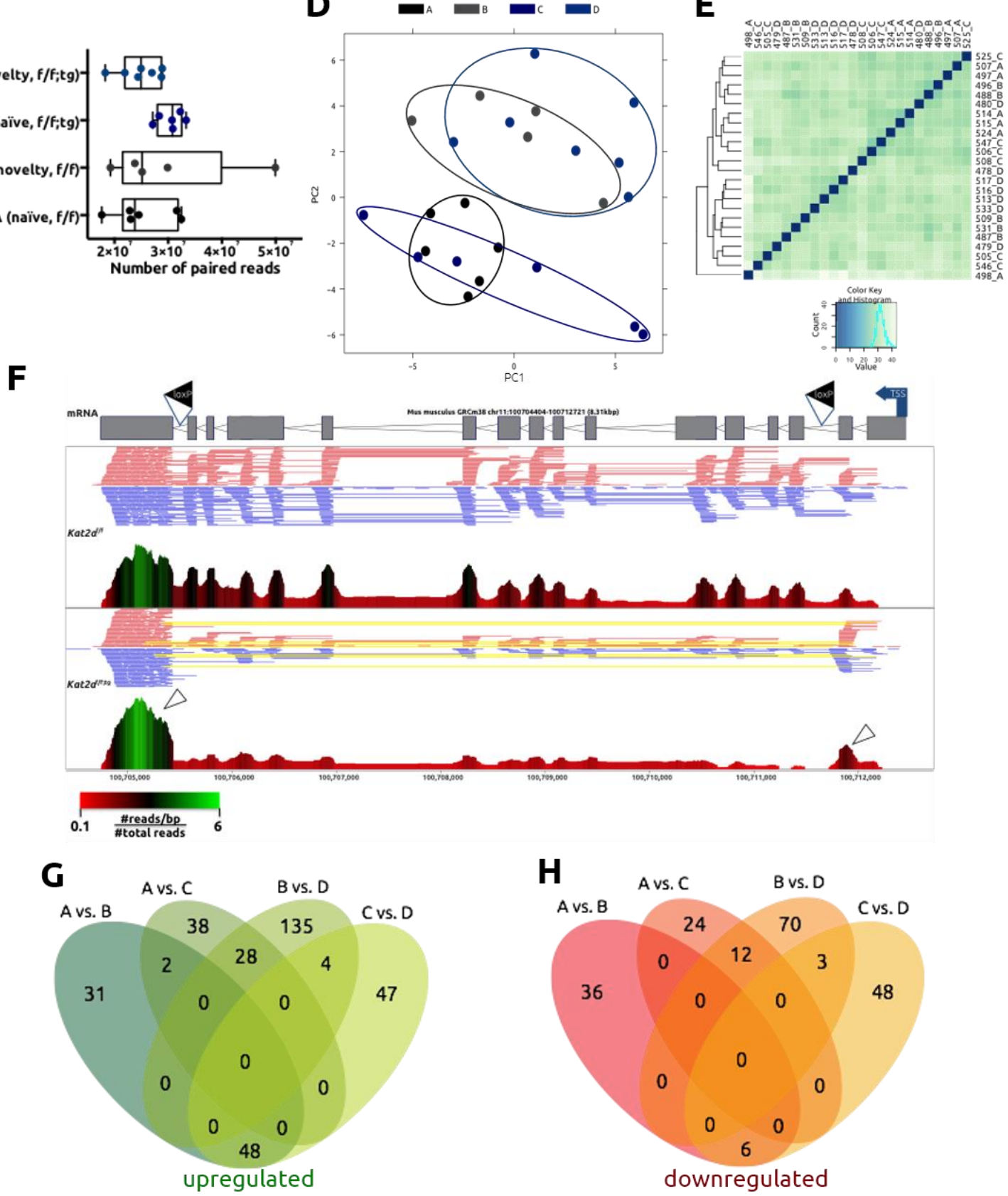

7 
spliced mRNA and are aligned to a genomic DNA sequence, this form of plotting allows to visualize reads that span intronic regions and thereby reveal connected exons. Indeed, within the sample of Kat2 $a^{\mathrm{f} / f ; \mathrm{ig}} \mathrm{mRNA}$ we could observe reads that span almost the entire transcribed region (yellow), except for exons 1 and 2 and the most-3' exon (arrowheads), which corresponds to the region deleted in the knockout condition. In fact, these exons seem to exhibit an even higher read count in the Kat $2 a^{f / f i t g}$ sample. Together, these observations confirm location of the loxP elements and even allow visualizing the transcript resulting from recombination between those (Figure 3-9F).

Using the R-based DEseq package to analyze differential expression for pair-wise comparisons in all possible combinations of the four groups (except combination $A$ vs. D), we found several differentially expressed genes (DEGs) with respect to novelty exposure, genotype or both ${ }^{19}$. We also found several DEGs overlapping between comparisons (Figure 3-9G,H and Table 3-4). Notably, most changes were found between group $B$ and $D$ (novelty-exposed, Kat $2 a^{\mathrm{f} / \mathrm{F}}$ vs. Kat $2 a^{\mathrm{f} / \mathrm{f} ; \mathrm{tg}}$ ), with 167 genes upregulated and 85 genes downregulated. Another notable observation is the extensive overlap between $A$ vs. $B$ and $C$ vs. $D$ (naïve vs. novelty for both genotypes) among the upregulated genes in contrast to downregulated genes (48 and 6, respectively).

\footnotetext{
${ }^{19}$ For a complete overview, sorted lists with all the DEGs found to be significantly up- or downregulated are given in a supplementary table in Appendix 6.2.1
} 
Table 3-4: Overlapping and non-overlapping DEGs. Below the description of each group a possible interpretation of that group with regard to Kat2a function is given. $\cap$ is the mathematical notation for "intersection".

\begin{tabular}{|c|c|c|c|c|}
\hline \multirow{2}{*}{ Group } & \multicolumn{2}{|r|}{ Upregulated } & \multicolumn{2}{|r|}{ Downregulated } \\
\hline & Total & Genes & Total & Genes \\
\hline $\begin{array}{c}\text { (A vs. B) } \cap \text { (A vs. C): } \\
\text { novelty-regulated, already changed } \\
\text { due to conditional knockout }\end{array}$ & 2 & Gm15583 Zscan20 & 0 & \\
\hline $\begin{array}{c}\text { (A vs. B) } \cap \text { (C vs. D): } \\
\text { novelty-regulated, likely } \\
\text { independent of Kat2a regulation }\end{array}$ & 48 & 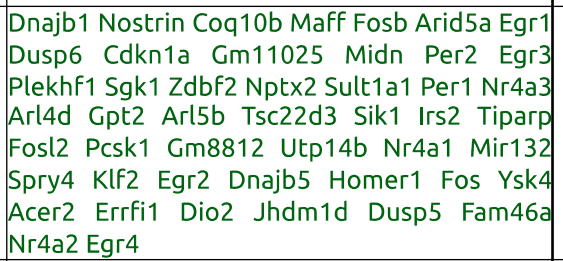 & 6 & $\begin{array}{l}\text { Plekho2 Egf Gm11458 Pkdrej Cd180 } \\
\text { Ppcs }\end{array}$ \\
\hline $\begin{array}{l}\quad(\mathbf{A} \text { vs. } \mathbf{C}) \cap(\mathbf{B} \text { vs. D): } \\
\text { regulated in knockout condition, } \\
\text { independent of novelty exposure }\end{array}$ & 28 & 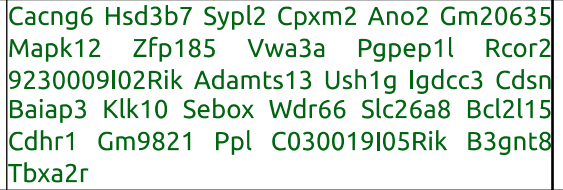 & 12 & $\begin{array}{l}\text { Cyp26b1 2410018L13Rik Npbwr1 Htr2a } \\
\text { Adcyap1 Grem1 Kat2a Fam19a1 Zfp934 } \\
\text { Gm3667 Oxr1 Penk }\end{array}$ \\
\hline $\begin{array}{l}\text { (B vs. D) } \cap \text { (C vs. D): } \\
\text { highly novelty-regulated in } \\
\text { knockout condition }\end{array}$ & 4 & Tnni3k 1700016P03Rik Atoh8 Ankrd53 & 3 & 4933413G19Rik Ehhadh Gm10574 \\
\hline $\begin{array}{c}\text { A vs. B only: } \\
\text { novelty-regulated, likely dependent } \\
\text { on Kat2a regulation }\end{array}$ & 31 & 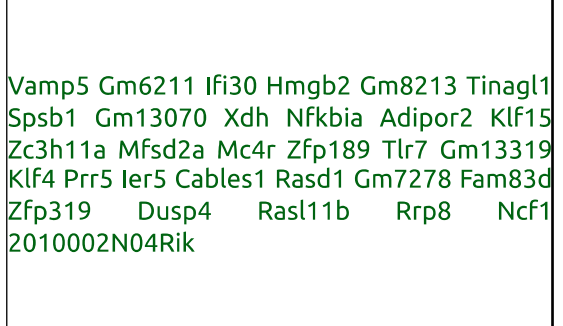 & 36 & $\begin{array}{llll}\text { Dcaf12l2 } & \text { 4632433K11Rik } & \text { Gm15817 } \\
\text { 4922502B01Rik } & \text { Glyat } & \text { C030005K06Rik } \\
\text { Gm8619 } & \text { Gm20692 } & 9130019022 R i k \\
\text { Gm9889 } & 1810059 H 22 R i k & \text { Tlr9 } & \text { Ccdc14 } \\
\text { Gm10345 } & \text { Gdf3 } & \text { Micall2 } & \text { BC024139 } \\
\text { Tmem194b Gm17096 AC151712.1 Hey } \\
\text { Gli2 } & \text { G530011006Rik } & \text { Fgf22 } & \text { Nt5dc1 } \\
\text { 4931417G12Rik } & \text { Klhl6 } & \text { Slc22a12 } & \text { Ccno } \\
\text { Atad5 Parp16 Gp1ba Mfsd10 BB218582 } \\
\text { Tnfrsf18 Gprin3 }\end{array}$ \\
\hline $\begin{array}{l}\text { A vs. C only: } \\
\text { regulated in knockout condition, } \\
\text { independent of novelty exposure }\end{array}$ & 38 & 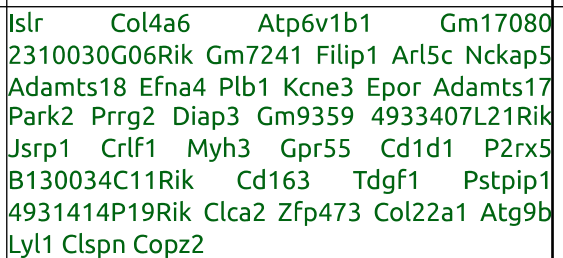 & 24 & 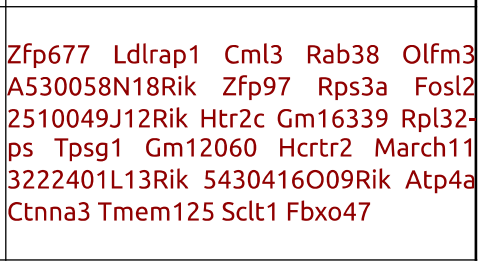 \\
\hline
\end{tabular}


Table 3-4 (continued)

\begin{tabular}{|c|c|c|c|c|}
\hline \multirow{2}{*}{ Group } & \multicolumn{2}{|r|}{ Upregulated } & \multicolumn{2}{|r|}{ Downregulated } \\
\hline & Total & Genes & Total & Genes \\
\hline $\begin{array}{l}\text { B vs. D only: } \\
\text { regulated in knockout condition, } \\
\text { dependent on novelty exposure }\end{array}$ & 135 & 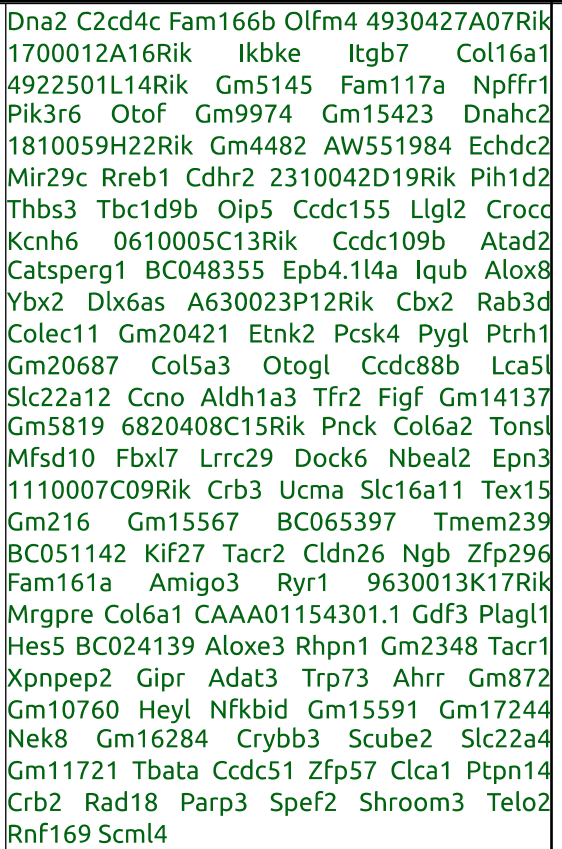 & 70 & 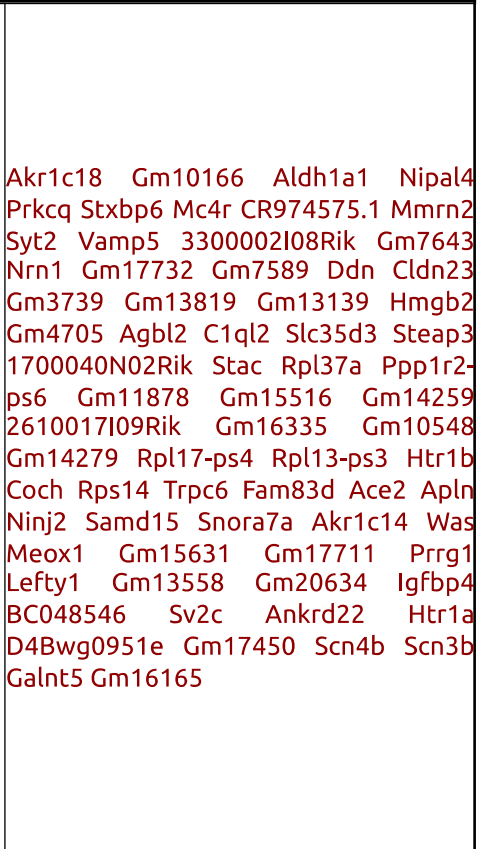 \\
\hline $\begin{array}{c}\text { C vs. D only: } \\
\text { novelty-regulated, likely } \\
\text { compensatory for Kat2a deficiency }\end{array}$ & 47 & 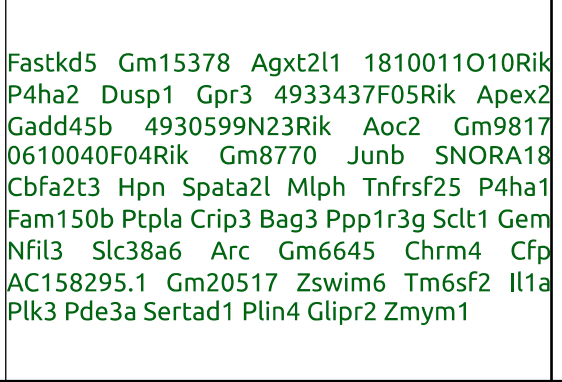 & 48 & 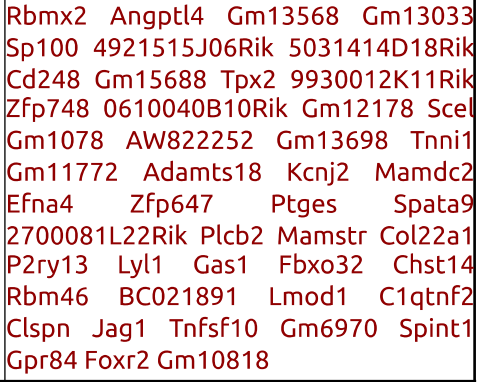 \\
\hline $\begin{array}{c}\text { Up(A vs. B) } \cap \text { Down(A vs. C) } \cap \\
\text { Up(C vs. D): } \\
\text { novelty-regulated, likely } \\
\text { independent of Kat } 2 \text { a regulation }\end{array}$ & 1 & Fosl2 & & \\
\hline $\begin{array}{c}\text { Up(A vs. B) } \cap \text { Down(B vs. D): } \\
\text { novelty-regulated, likely dependent } \\
\text { on Kat2a regulation }\end{array}$ & 4 & Vamp5 Hmgb2 Mc4r Fam83d & & \\
\hline $\begin{array}{l}\text { Down(A vs. B) } \cap \text { Up(B vs. D): } \\
\text { novelty-regulated, likely inversely } \\
\text { or indirectly regulated by Kat } 2 a\end{array}$ & 7 & 1810059H22Rik Slc22a12 Mfsd10 Heyl Ccno Gd & $\mathrm{AF} 3 \mathrm{BC}$ & C024139 \\
\hline $\begin{array}{l}\text { Up(A vs. C) } \cap \text { Down(C vs. D): } \\
\text { increased due to knockout, but } \\
\text { decreased upon novelty-exposure, } \\
\text { likely independent of Kat2a } \\
\text { regulation, possibly compensatory }\end{array}$ & 6 & Adamts18 Lyl1 Clspn Efna4 Col22a1 & & \\
\hline $\begin{array}{l}\text { Down(A vs. C) } \cap \text { Up(C vs. D): } \\
\text { decreased due to knockout, but } \\
\text { increased upon novelty-exposure, } \\
\text { likely independent of Kat } 2 a \\
\text { regulation, possibly compensatory }\end{array}$ & 1 & Sclt1 & & \\
\hline
\end{tabular}


To further examine potential functional relevance of these DEGs, we performed functional annotation by searching for overrepresentation of genes associated with specific functional categories (GO-Terms) or pathways (KEGG-Pathways). A complete overview of all such enriched categories can be found in section 6.2.2, Table 6-1 through Table 6-4). Here, I will focus on general trends that help to interpret the results.

To study the effect of Kat2a deletion in naïve mice, groups $\boldsymbol{A}$ and $\boldsymbol{C}$ need to be compared. Here, plasma-membrane-associated genes were found among the upregulated DEGs. Genes related to activation of phospholipase $\mathrm{C}$ activity and neuroactive ligand-receptor interaction were enriched in downregulated DEGs.

When comparing naïve with novelty-exposed mice, prominent upregulation of transcriptionregulation associated genes was found for either genotype ( $A$ vs. $B$ and $C$ vs. $D$ ). Among downregulated genes, those associated with growth-factor activity were enriched only in Kat2 $a^{\mathrm{f} / \mathrm{f}}$ mice, while in Kat2 $a^{f / f ; t g}$ mice genes that were downregulated upon novelty exposure were not enriched in any specific functional categories. (Table 6-1 and Table 6-4). Novelty also induced a significant number of genes involved in MAPK/ERK signaling in both genotypes.

When comparing stimulus-dependent gene expression between $K a t 2 a^{f / f ; t g}$ and control mice, we found an overrepresentation of upregulated genes that were associated with calcium-ion binding and extracellular processes. Strikingly, genes involved in neuronal activities such as serotonin receptor activity were enriched among downregulated genes. Additionally, genes associated with the ribosome, and therefore translation, were found. When we used the 135 genes that were upregulated exclusively in $B$ vs. $D$ we did neither find any evidence for increased enrichment of those categories aforementioned nor of any additional categories.

In conclusion, we identified a well-defined group of genes that respond to novelty exposure in the CA1, independent of Kat2a status. This group mainly consists of immediate early genes (IEGs) and transcriptional regulators. However, we also found several genes that show a differential response to a novelty stimulus, depending on genotype. This set of genes, together with those genes differentially expressed to Kat2a deletion in the naïve condition, are likely candidates for direct or indirect Kat2a regulation. Interestingly, several of these genes are associated with neuronal activity and translation. The significance and function of several of the identified genes are discussed in 4.1.2. 


\subsubsection{Kat2a Conditional Knockout Mice Show Increased Levels of H4K12 Acetylation}

To investigate, whether Kat2a loss-of-function results in altered histone acetylation in the hippocampus, we analyzed bulk levels of acetylation in whole hippocampal tissue for H4K12, H3K9 and H3K14 by western blot. These are the lysine residues associated with Kat2a function in the GOTerm database, based on findings in the literature. To our surprise, bulk levels of H4K12ac were found to be elevated almost 2-fold in Kat2 $a^{\mathrm{f} / \mathrm{F} \text { tg }}$ mice, while H3K9ac and H3K14ac did not show significant changes (2way ANOVA had significant effect of genotype: $F_{(1,18)}=9,08, p=0.008$, Šidák's multiple comparisons test showed significant differences in H4K12ac: $p_{a d j}=0.008$; Figure 3-10).

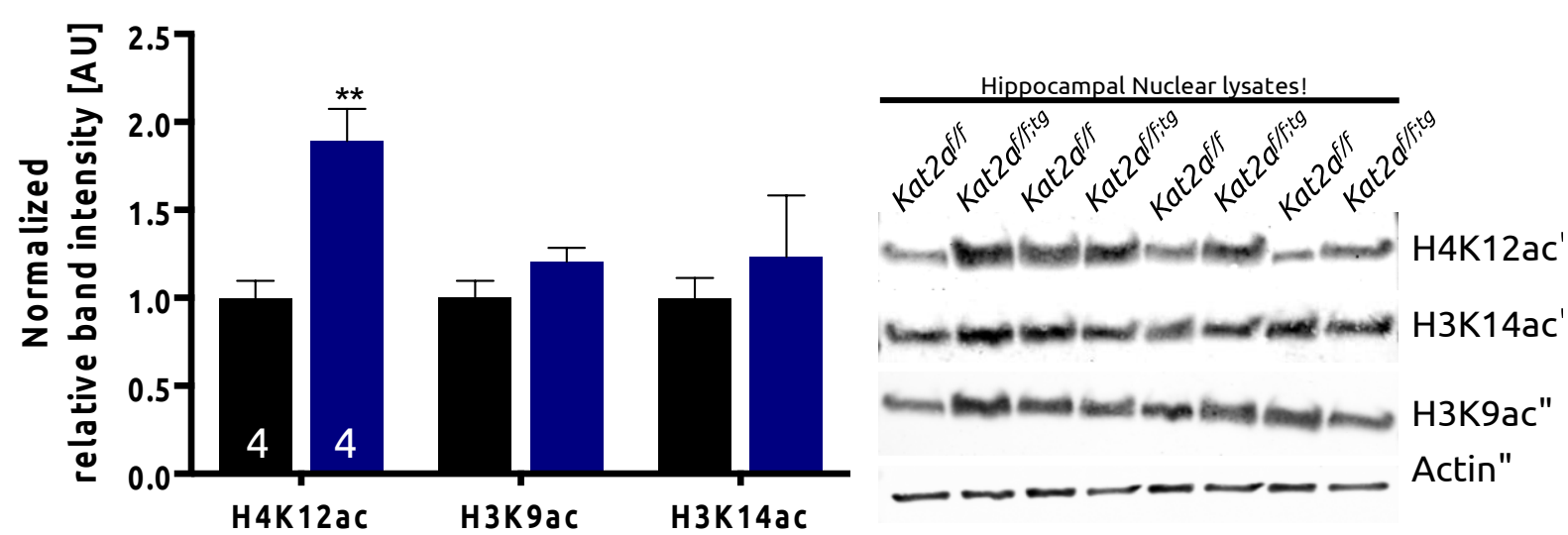

Figure 3-10: Bulk levels of histone acetylation in the hippocampus of $K a t 2 a^{\mathrm{f} / F ; t g}$ and control mice. H4K12ac was significantly increased whereas H3K9 and H3K14 showed only a trend towards elevated acetylation levels. Asterisks indicate significant difference according to Šidák's multiple comparisons test. 


\subsubsection{Characterization of AAV-mediated Neuron-Specific Kat2a Overexpression in the Dentate Gyrus}

After investigating the role of Kat2a using a loss-of-function approach, we wanted to study Kat2a gain-of-function. Overexpression studies offer certain advantages over knockout studies, since they allow greater focality and specificity in many respects.

As we found Kat2a mRNA to be expressed strongest in the CA region, which is supported by data from IN SITU hybridization experiments in the Allen brain atlas, we determined the dentate gyrus (DG) to be a promising region to study effects of Kat2a overexpression. To this end, we made use of adeno-associated viral (AAV) vectors, which allows focal, neuron-specifc expression of MYC-tagged Kat $2 \mathrm{a}^{20}$ under control of the human SYN1 promoter. ${ }^{21}$ For these experiments, male WT C57BL/6J mice were used.

\subsubsection{Robust AAV-mediated Expression of MYC-tagged Kat2a 14 Days after Injection}

Using a MYC-specific antibody for immunohistochemistry of cryosections retrieved at 5 different time points after injection, we found initial expression of Kat2a-MYC at about 10 days. Robust expression across the whole DG was evident at 14 days after intra-hippocampal injection of viral particles (Figure 3-11A). After 14 days Kat2a mRNA levels had increased almost 5-fold compared to GFP-control condition as well as to AAV-mediated Hdac2 overexpression (Figure 3-11B) ${ }^{22}$. This was in line with other overexpression studies, using AAV vectors (Bahari-Javan et al., 2012) and was also confirmed when we overexpressed Hdac2-MYC for control purposes (see Figure 6-2).

\footnotetext{
${ }^{20}$ Two known isoforms are expressed form Kat2a locus. The one used here was: NCBI: isoform b / variant 2 , NM_001038010.2; ENSEMBL: Kat2a-001, ENSMUST00000006973; see 6.3B for vector map)

${ }^{21}$ The viral particles were generated at the University Medical Center Göttingen in close collaboration with Dr. Sebastian Kügler and colleagues.

${ }^{22}$ For this experiment mRNA was extracted from the whole hippocampus, not from DG specifically.
} 
A
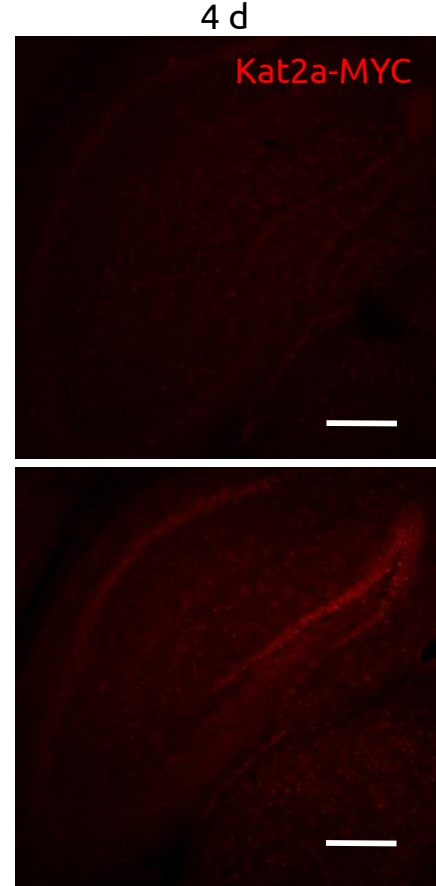

$12 \mathrm{~d}$
$7 \mathrm{~d}$
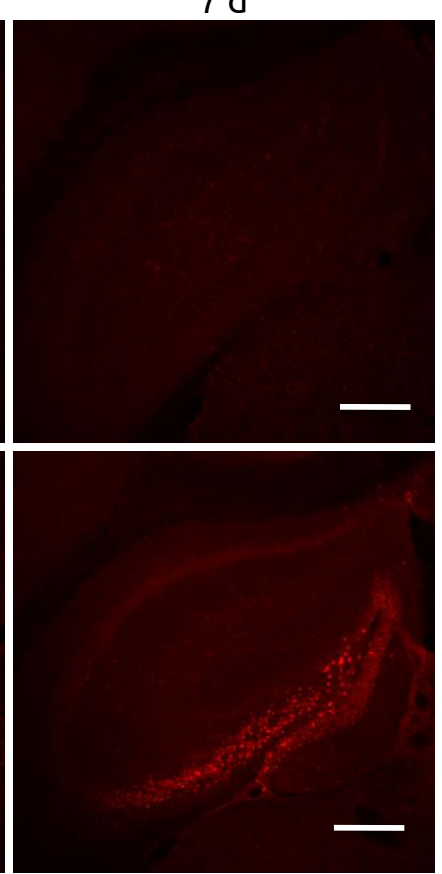

$14 \mathrm{~d}$
$10 d$
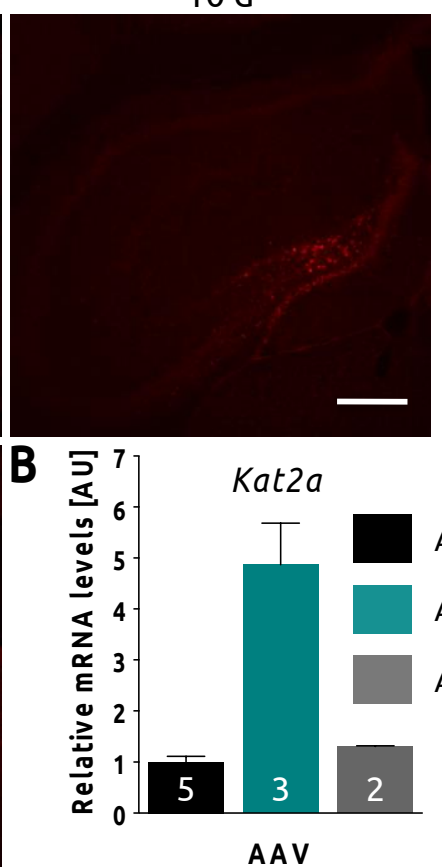

AAV-GFP

AAV-Kat2a-MYC

AAV-Hdac2-MYC

Figure 3-11: AAV-mediated expression of Kat2a in the dentate gyrus. First evidence of expression was observed at 10 days, robust staining at 14 days after injection (A). Kat2a mRNA levels increased about 5-fold at this time in mice injected with Kat2a-MYC-AAV compared to mice injected with either GFP-AAV or Hdac2-MYCAAV (B). Representative images, scale bar is $250 \mu \mathrm{m}$.

\subsubsection{Kat2a Overexpression Results in Spatial Memory Impairment}

To test for behavioral effects of Kat2a overexpression in the dentate gyrus, AAV-Kat2a-MYCinjected mice underwent behavioral testing, starting 14 days after AAV administration. Mice overexpressing GFP only under otherwise equal conditions served as control group. This time, the behavioral test battery did not include Rotarod performance testing nor the visual form of the MWM, since the parameters measured by these test were most likely not affected by focal overexpression of Kat2a, as opposed to the forebrain-wide-knockout condition.

Starting with an open field test, we first tested for altered exploratory behavior and locomotion. We found both groups spending comparable amounts of time in the center region of the arena and traveling similar distances within 5 min. Also average traveling speed was similar (Figure 3-12A).

In the elevated plus maze anxiety and exploratory behavior was reviewed. Both groups showed normal levels of anxiety, as they spent most of the time $(\sim 85-95 \%)$ in the closed arms or center region. Hence, there was no difference in time spent in the open arms of the maze (Figure 3-12B).

By submitting both groups to explore a 4-arms cross maze, working memory was probed by monitoring the sequence of arm entries. Again, successful alterations were defined as visits of an arm that was not visited within the last three trials before. Both groups performed significantly better than the $9.375 \%$-chance level, indicating non-random arm entries. (Student's one-sample $t$ test: AAV-GFP: $p=0.001$, AAV-Kat2a-MYC: $p<0.001)$. However, there was no significant difference between groups (Figure 3-12C) 
A novel object recognition (NOR) paradigm was used to determine potential effects on short-term or long-term recognition memory. After habituation, the AAV-GFP group showed no preference for any of the two equal objects during the training session, while the AAV-Kat2a-MYC group demonstrated some preference for the object that would later not be replaced for the new one (Student's one-sample t-test vs. 0.5: $p=0.027$; Figure 3-5D, TR). Both groups however showed equivalent preference for the novel object in the short-term memory paradigm (Student's onesample t-test vs. 0.5: p=0.005 for both groups; Figure 3-12D, STM). Interestingly, in the long-term paradigm, none of the groups showed significant preference for the novel object (Student's onesample t-test vs. 0.5: AAV-GFP: $p=0.578$, AAV-Kat2a-MYC: $p=0.129$; Figure 3-12D, LTM).

Also here, we tested associative learning in the contextual fear-conditioning (cFC) paradigm. While baseline freezing in the training situation was equivalently low, AAV-Kat2a- mice showed a nonsignificant tendency towards more freezing behavior when reintroduced to the context 24 hours after receiving the electric foot shock (Figure 3-12E, right panel). To exclude effects of altered sensory perception, we also monitored shock response activity during the 2s-shock phase (Figure 3-12E, right panel). 
A

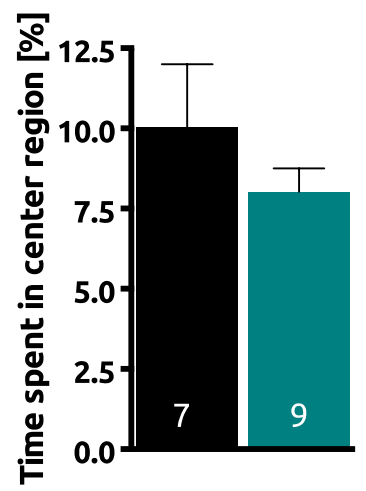

Open Field Test

B

Elevated Plus Maze

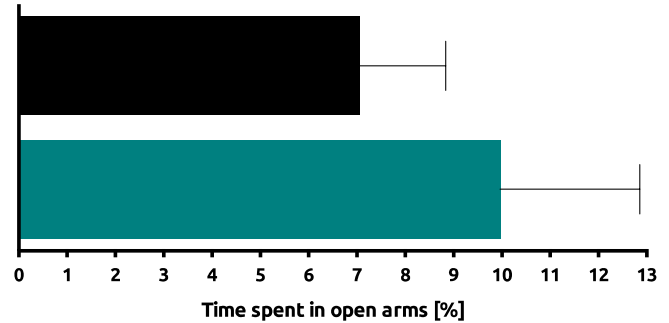

C

Cross Maze
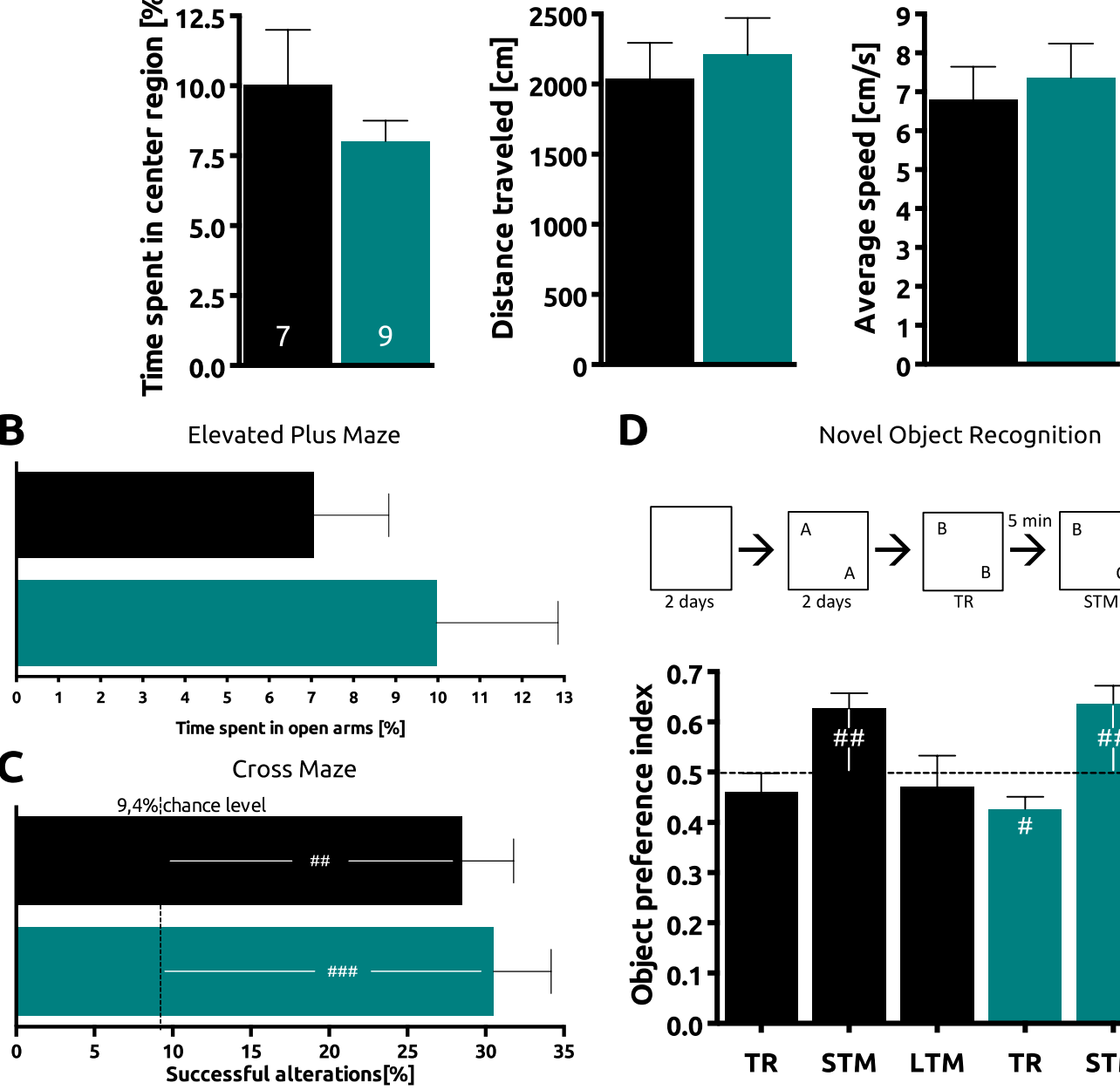

D

Novel Object Recognition
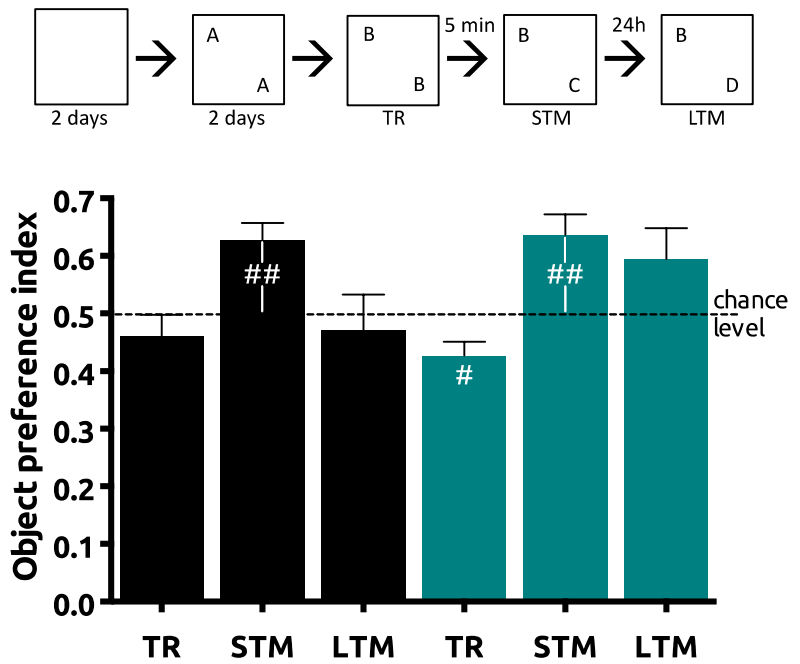

E Contextual Fear Conditioning
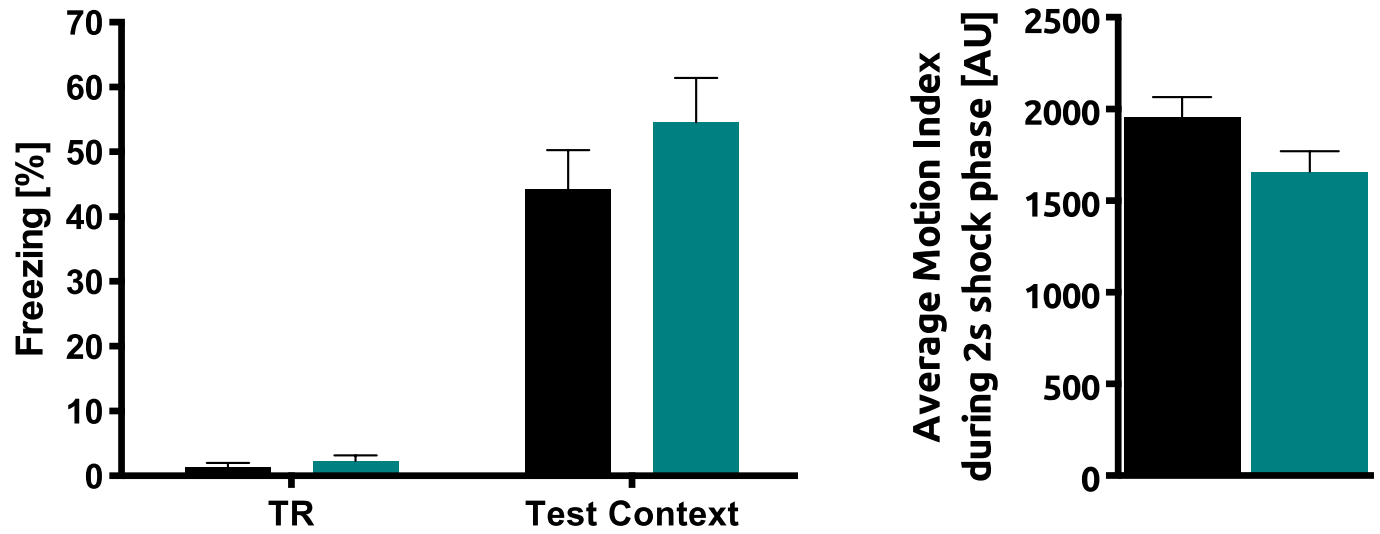

AAV-GFP

AAV-Kat2a-MYC

Figure 3-12: Behavioral analysis of Kat2a gain-of-function. Open field test (A), Elevated plus maze (B) and 4arms cross maze (C) indicated normal behavior with regard to locomotion, exploratory behavior, basal anxiety and working memory. Novel object recognition (D) and contextual fear conditioning (E) showed an insignificant trend towards increased long-term memory. Control parameters like object preference during training (D, TR) or activity in response to electric foot shock (E, right panel) were only slightly different. AAV-GFP served as control group. Hashes indicate significant differences from chance level within groups. TR, Training; STM, Short-term memory; LTM, Long-term memory. For all experiments: $n=7$ (AAV-GFP), $n=9$ for (AAV-GFP). 
Finally, we subjected mice to the MWM. Though both groups rapidly learned to navigate within the pool $^{23}$, AAV-Kat2a-MYC mice displayed a significant retardation in finding the hidden platform (rmANOVA, main effect of time: $F_{(6,84)}=33.8, p<0.001$; main effect of AAV: $F_{(1,14)}=7.9, p=0.014$; Fisher's uncorrected LSD test for multiple comparison : Day2, $p=0.028$; Day3, $p=0.022$; Day7, $p=0.009$; Figure 3-13A).

At the day of the probe test, the platform was removed and time spent in the four quadrants of the pool was monitored for one minute. Time spent in the target quadrant (TQ) was almost significantly different from chance level (Student's one-sample t-test: $p=0.0549$ ) as well as from average time spent in the other three quadrants ( 1 1-3, Student's t-test: $p=0.0548$ ) in AAV-GFP mice (Figure 3-13B). However, AAV-Kat2a-MYC mice did not show any evidence for preference for the target quadrant. Also the number and integrated time of crossing over the former platform region was reduced in AAV-Kat2a-MYC mice, though the difference in platform crossings was only at the verge of statistical significance (Student's t-test: Platform crossings: $p=0.063$, Time: $p=0.029$, Figure 3-12C,D). To exclude effects of potential mobility impairments to account for this differences, we also monitored the swim speed on the probe test day), in order to get an average over a full minute of swimming behavior (Figure 3-13E). We further excluded the possibility of differential body weight to affect the results of behavioral testing, as both groups had comparable body weights after completing the behavioral test battery (Figure 3-13F)

In conclusion, Kat2a gain-of-function did neither result in changed locomotion or baseline anxiety levels nor was there any evidence for altered working memory or short-term recognition memory. Interestingly, long-term memory was affected in both groups. Though there was a trend towards increased aversive memory in the CFC paradigm, spatial memory impairments became evident in the MWM.

\footnotetext{
${ }^{23}$ In this experiment both groups were notably fast to learn how to escape the watermaze, especially the control group. In order to be able to still pick up potential differences between AAV-GF and AAV-Kat2a-MYC mice, we stopped training phase after day 7 and performed probe testing on day 8.
} 
A

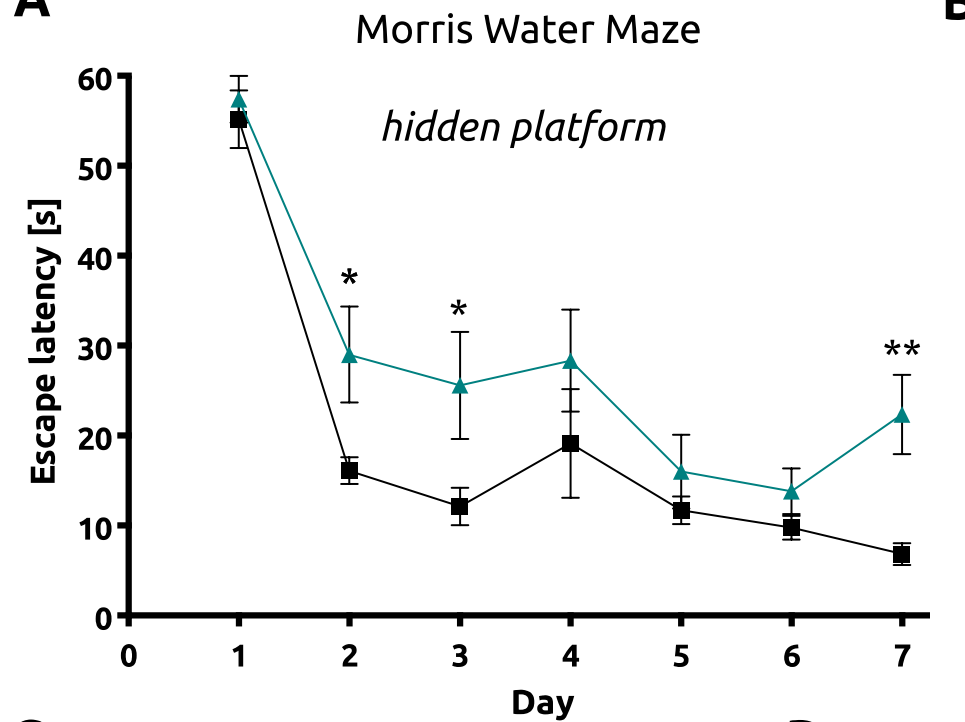

B

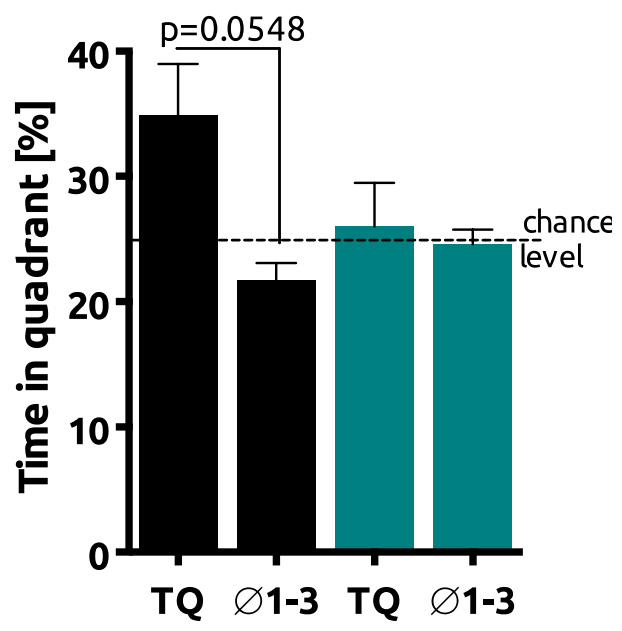

C

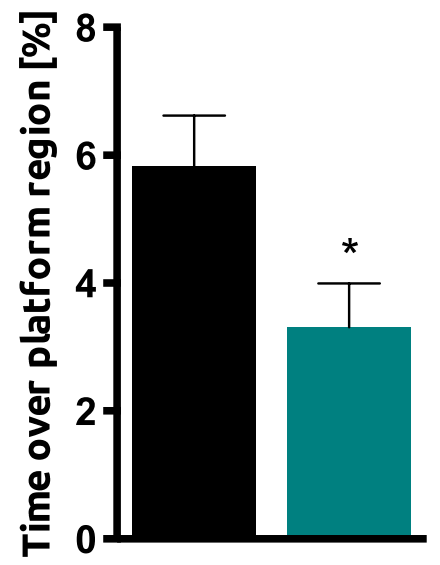

E

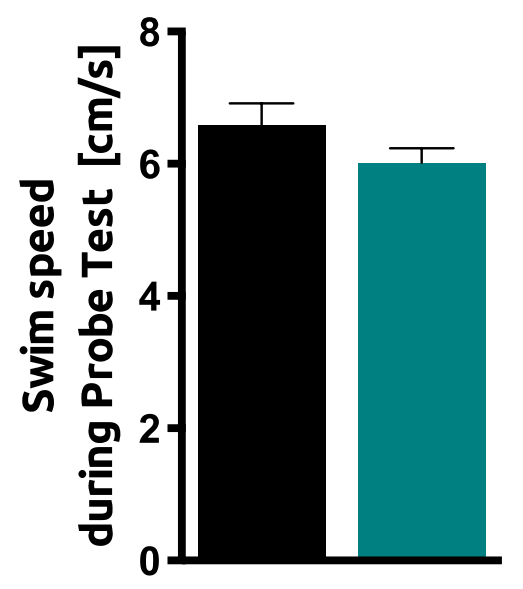

D

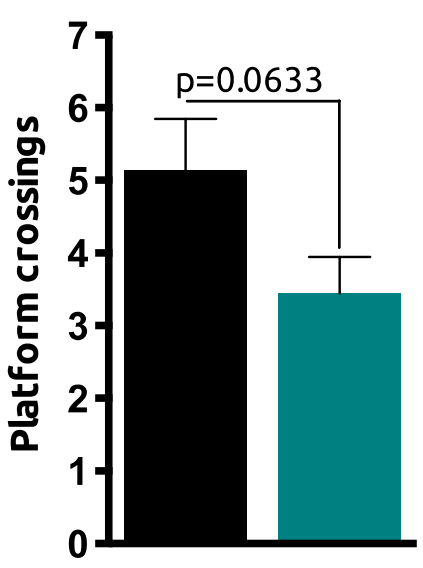

$\mathbf{F}$

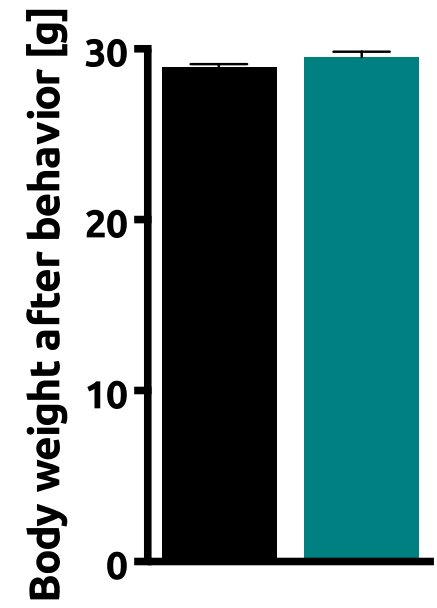

AAV-Kat2a-MYC

Figure 3-13: Morris water maze reveals impairment in spatial memory in Kat2a-overexpressing mice. Learning curves demonstrate rapidly decreasing escape latencies for both groups (A). Time spent in each of the four quadrants on the day of the probe test (B). Summed time spent over former platform region and number of platform crossings during probe test $(C, D)$. Average swim speed during probe test (E). Body weight as measured for both groups after completion of behavioral battery (F). Asterisks indicate significant differences between groups. TQ, Target quadrant; 1-3, quadrants 1 to 3 . Indicated p-values are based on Student's t-test. 


\subsection{Regulation of Gene Expression in Hippocampal Aging}

Regulation of gene expression is an important step in maintaining homeostasis. Aging can be viewed as a process leading to deterioration of homeostasis. Therefore we were interested in the transcriptional regulation in the aging hippocampus and its association with potential alterations in histone acetylation, with a special focus on advanced aging. For all following experiments, only male C57Bl/6J mice were used.

\subsubsection{Histone Acetylation in the Aged Hippocampus}

\subsubsection{Decreased HDAC Activity is not Associated with Bulk Changes in Histone Acetylation Levels in} the Aged Hippocampus

Middle-aged (16-month-old) mice failed to induce upregulation of H4K12ac upon a learning stimulus and subsequent regulation of learning-related genes. This induction could be reinstated using HDACi. Interestingly, naïve mice of this age did neither show alterations in histone acetylation nor in nuclear HDAC activity (Peleg et al., 2010).

Based on these results we asked, whether advanced aging may be associated with changes in HDAC or HAT activity and ultimately histone acetylation in a mouse model for advanced aging. To this end, we measured these parameters in 24-month-old mice and mice of an even more advanced age ( $\geq 28$ months), to explore effects that occur towards the end of lifespan (EOL) of these animals. For all these assays, nuclear lysates prepared from hippocampal tissue were used. It is important to reiterate this here, since the procedure of subcellular fractionation precludes potential effects of non-nuclear-localized HDACs, HATs and importantly also histones that are synthesized in the cytoplasm and may already carry post-translational modifications.

Remarkably, we observed decreased HDAC activity with increasing age. 1way ANOVA revealed a significant effect of age $\left(\mathrm{F}_{(2,17)}=8.1, \mathrm{p}=0.003\right)$ and a significant linear trend between HDAC activity and age $\left(R^{2}=0.473, p=0.001\right)$. The age effect was attributed to a significant difference between 3month-old control mice with $\geq 28$-month-old EOL mice (Tukey's multiple comparisons test: $p=0.003$, Figure 3-14A). HAT activity however, remained unchanged. Here, we monitored kinetic activity development over time and found a significant effect of time, but not of age (rmANOVA, main effect of time: $F_{(16,304)}=2736, p<0.001$, Figure 3-14B).

The assay for HDAC activity measurement is unselective towards a specific HDAC. In order to determine, whether a particular HDAC (or HDAC family) is responsible for the detected decrease in activity in 3 vs. $\geq 28$-month-old mice, we performed quantitative western blotting for Hdac2 and Hdac3, two HDACs that have been implicated in memory function before (Guan et al., 2009; McQuown and Wood, 2011). However, we did not detect alterations in nuclear protein levels of these HDACs (Figure 3-14C).

We next asked, whether decreased HDAC activity is associated with altered histone acetylation. As representatives we chose H3K9, H3K14 and H4K12 as potentially deregulated target sites. Interestingly, using quantitative western blots we could detect slightly increased bulk levels at all 
three marks, when related to total histone $\mathrm{H} 3$ or $\mathrm{H} 4$ levels. However, these differences were not statistically significant.

Taken together, increasing age is associated with decreasing HDAC activity, but this was not explained by a reduction in protein levels of Hdac2 or $\mathrm{Hdac} 3$ and paralleled by only insignificantly increased bulk histone acetylation at H3K9, H3K14 or H4K12 sites.

A

HDAC activity assay

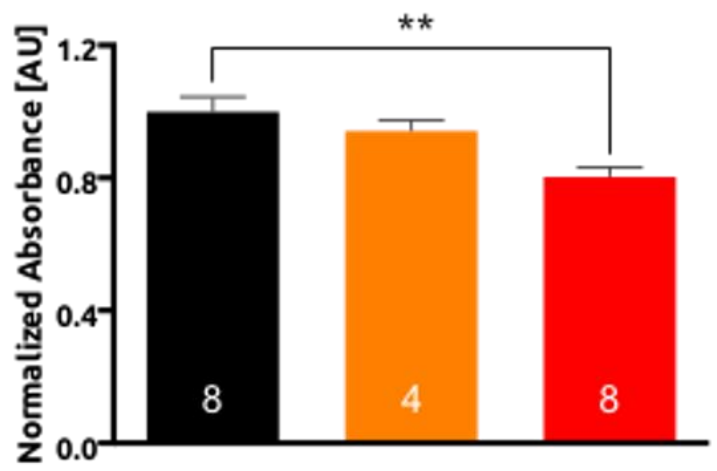

C

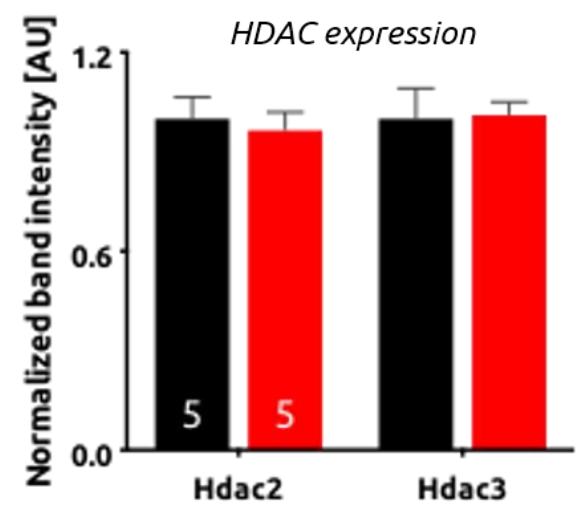

B

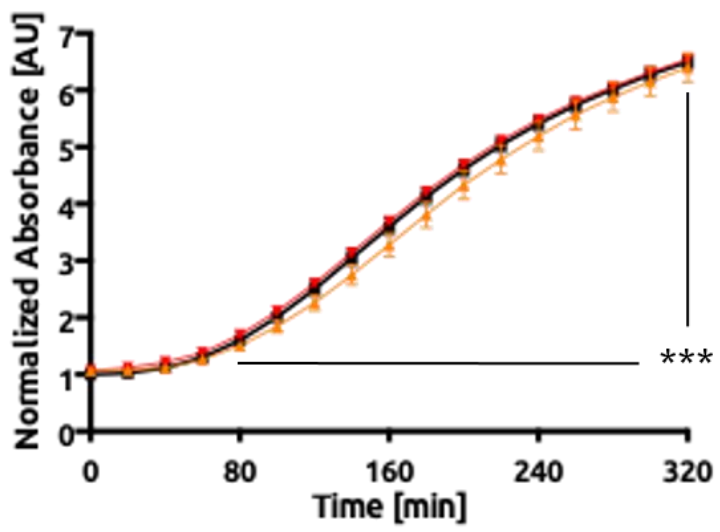

HAT activity assay

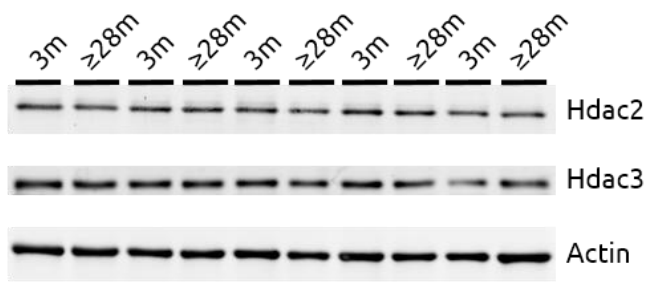

D

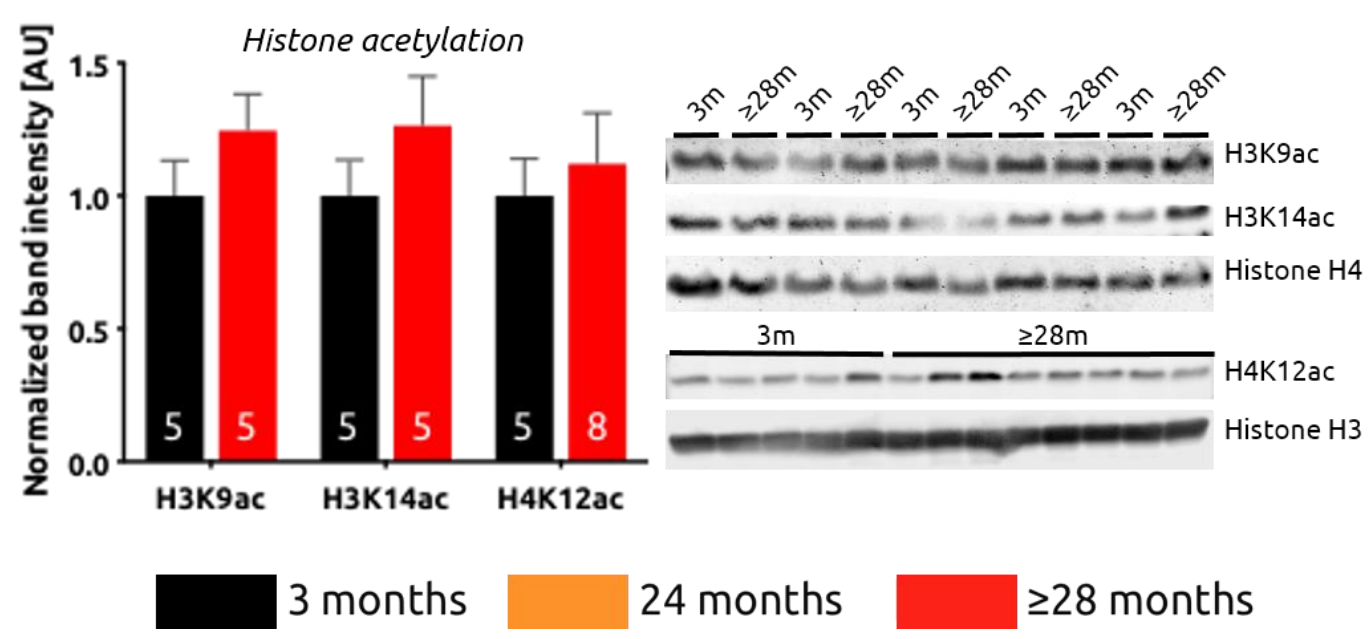

Figure 3-14: EOL mice exhibit decreased nuclear HDAC activity, but no altered bulk histone acetylation. HDAC activity followed a linearly decreasing trend with increasing age. When comparing 3-month old mice with $\geq 28$-month-old mice the trend reached statistical significance (A). HAT activity kinetics were similar between age groups (B). Hdac2 and Hdac3 protein levels were did not change between young and EOL mice(C). Acetylation of three histone sites was not changed significantly (D). 


\subsubsection{Orally Administered HDAC Inhibition Improves Age-Associated Memory Impairment}

Based on these results, we assumed a compensatory mechanism to act in the hippocampus with advancement of aging. We therefore hypothesized that facilitating this naturally occurring process may improve aging-associated memory impairment (AAMI). In favor of this notion, the host laboratory could recently show, that administration of the HDAC inhibitor SAHA restored spatial and aversive memory in aged (16 months old) wild type mice, when infused intrahippocampally. Based on these findings, we hypothesized that pharmacological HDACi may have a beneficial effect on memory also at an advanced age.

In a pilot experiment, we asked the question, whether systemic administration of SAHA would have an effect on histone acetylation in the brain, especially in the hippocampus. To answer this question we injected 3-month-old mice with either vehicle or $50 \mu \mathrm{g} / \mathrm{g}$ of SAHA INTRA PERITONEAL (IP) once per day over the course of 7 days. After the last injection, we isolated brain tissue of mice after $1,3,8$ and 24 hours and measured H4K12 acetylation by western blotting.

Importantly, we discovered significant elevation of H4K12ac levels only at 1 hour after the last injection (Student's t-test: $p=0.029$; Figure 3-15). This increase was absent already 2 hours later.

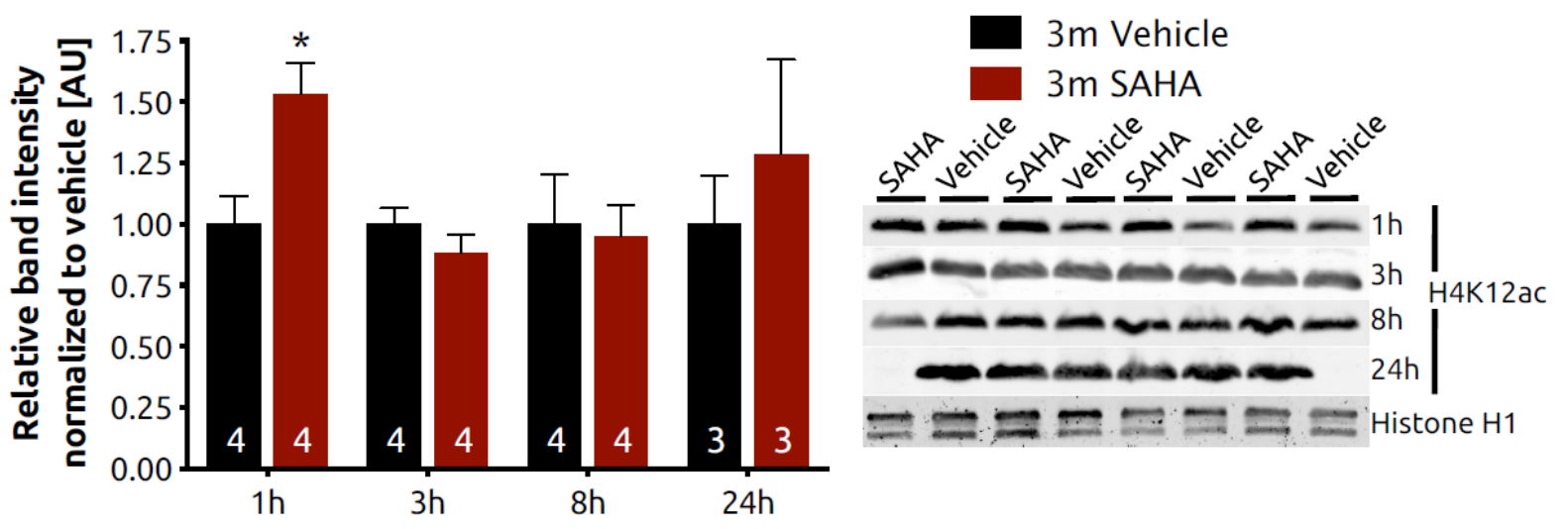

Figure 3-15: Chronic SAHA administration in young mice transiently increases H4K12 acetylation. One hour after the last injection, H4K12ac levels were significantly elevated, and were indistinguishable from vehicle controls beyond 3 hours. 
To explore putatively beneficial effects of SAHA in aged mice, we fed 23-month-old mice for 3 weeks with $50 \mu \mathrm{g} / \mathrm{g}$ body weight of SAHA as explained in 2.2.2 and subsequently started behavioral testing. Oral administration was chosen over daily i.p. injections, since mice of this advanced age are considerably more fragile compared to young, healthy mice and the stress of repeated fixation and injection may have resulted in a high premature death rate. Furthermore, with an eye on potential clinical applications in the future, oral administration is more favorable compared to any form of injection.

Because in our experience working with 24-month-old mice leads to problems during elevated plus maze, Rotarod performance test, MWM and cFC, these tests were not conducted for the purpose of this study. However, we did observe normal behavior in the OF test in terms of time spent in the center region, which is a measure for exploratory behavior and basal anxiety. This parameter was comparable between groups fed with SAHA and vehicle as were the other parameters measured, namely traveled distance and average speed (Figure 3-16A).

The NOR test is a powerful memory test, in that it allows to compare recognition memory not only between groups, but also within a group, as the object preference parameter offers to test VERSUS randomness (i.e. chance level). Here, we used a slightly modified novel object recognition paradigm in order to meet previous observations that mice of advanced ages lack motivation to explore objects when habituated for a long time. During the training session, both groups explored each of the similar objects equivalently. Also in the 5-min-STM test, mice of neither the SAHA group nor vehicle group did show preference for any of the two objects. Interestingly, while vehicle treated mice still had no preference for either object after reintroduction for the 24-hours-LTM test, SAHA treated mice showed a moderate, but statistically significant preference for the novel object (Student one-sample t-test: $p=0.047$; Figure 3-16B).

As mice were fed with $200 \mathrm{mg}$ of high-caloric peanut butter, mixed with $20 \mu$ of either vehicle or SAHA solution, we also monitored potential changes in body weight. The first observation was readiness of peanut butter uptake: Mice ingested the prepared mixture without hesitation and within a time of only 10 minutes. Interestingly, this devouring of high-fat extra diet during 3 weeks did neither lead to overt changes in body weight within groups nor was there an effect of the type of additive (SAHA or vehicle). Though rmANOVA revealed a significant effect of time $\left(\mathrm{F}_{(10,170)}=5.3\right.$, $p<0.001$ ), this effect was attributed to individual daily fluctuations (e.g. POST HOC test for day 5 vs. day 13, Tukey's multiple comparisons test: $p=0.0012$ ) and there was no significant difference between day 1 and day 21 (Figure 3-16C). 
A

Open Field Test
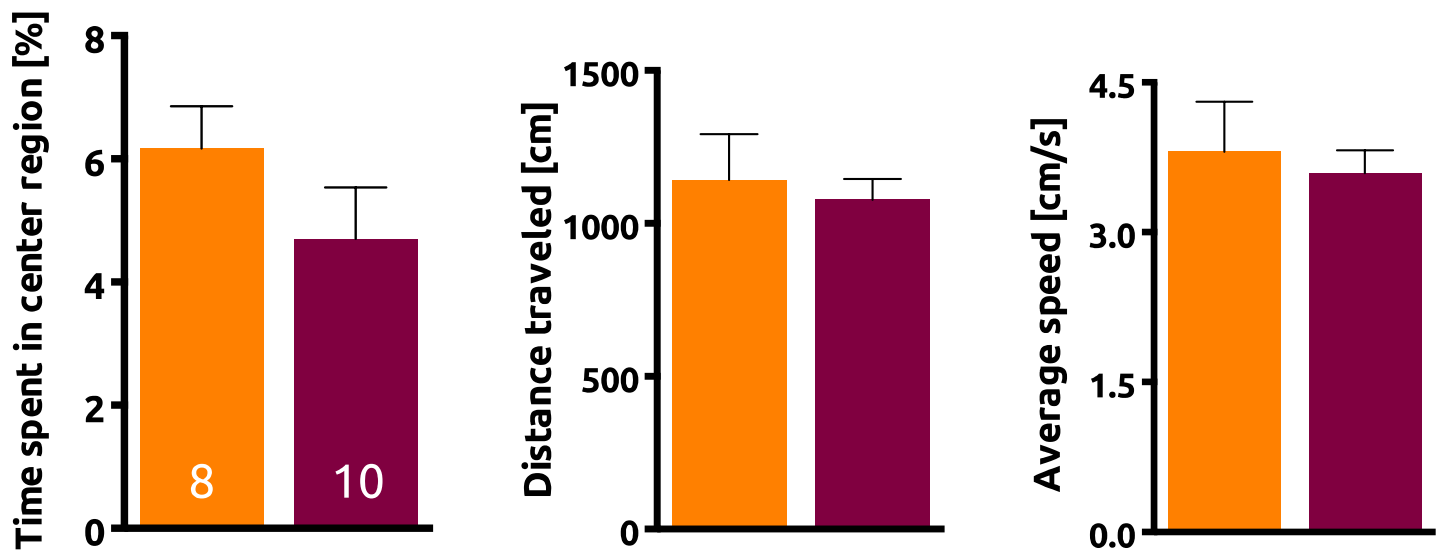

B

Novel Object Recognition
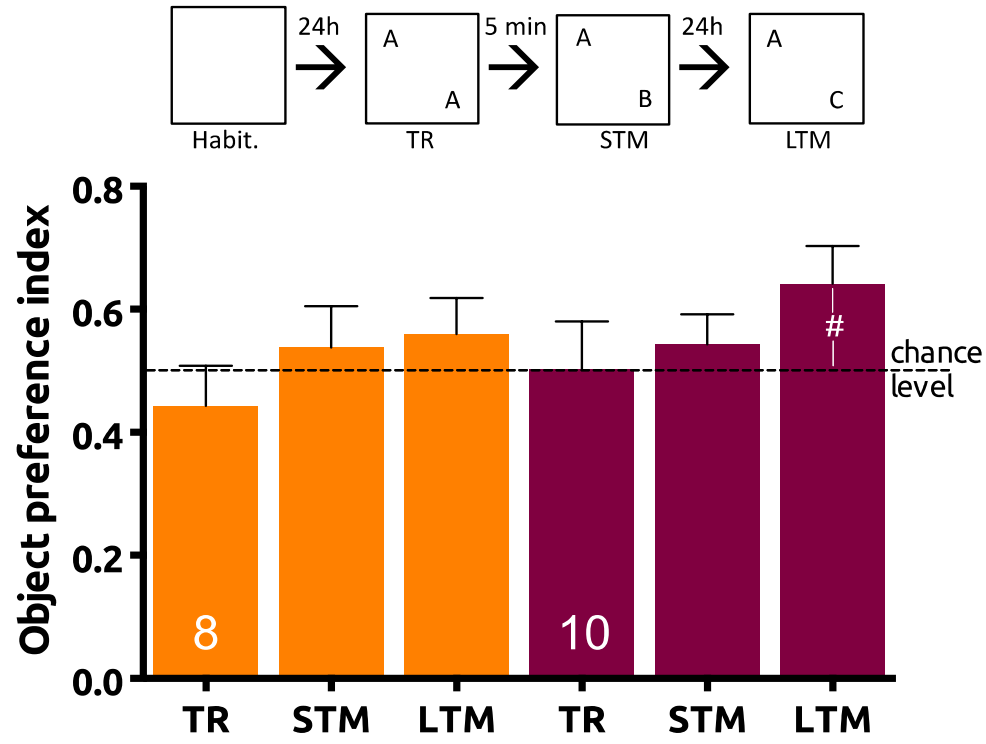

24m Vehicle

24m SAHA

C

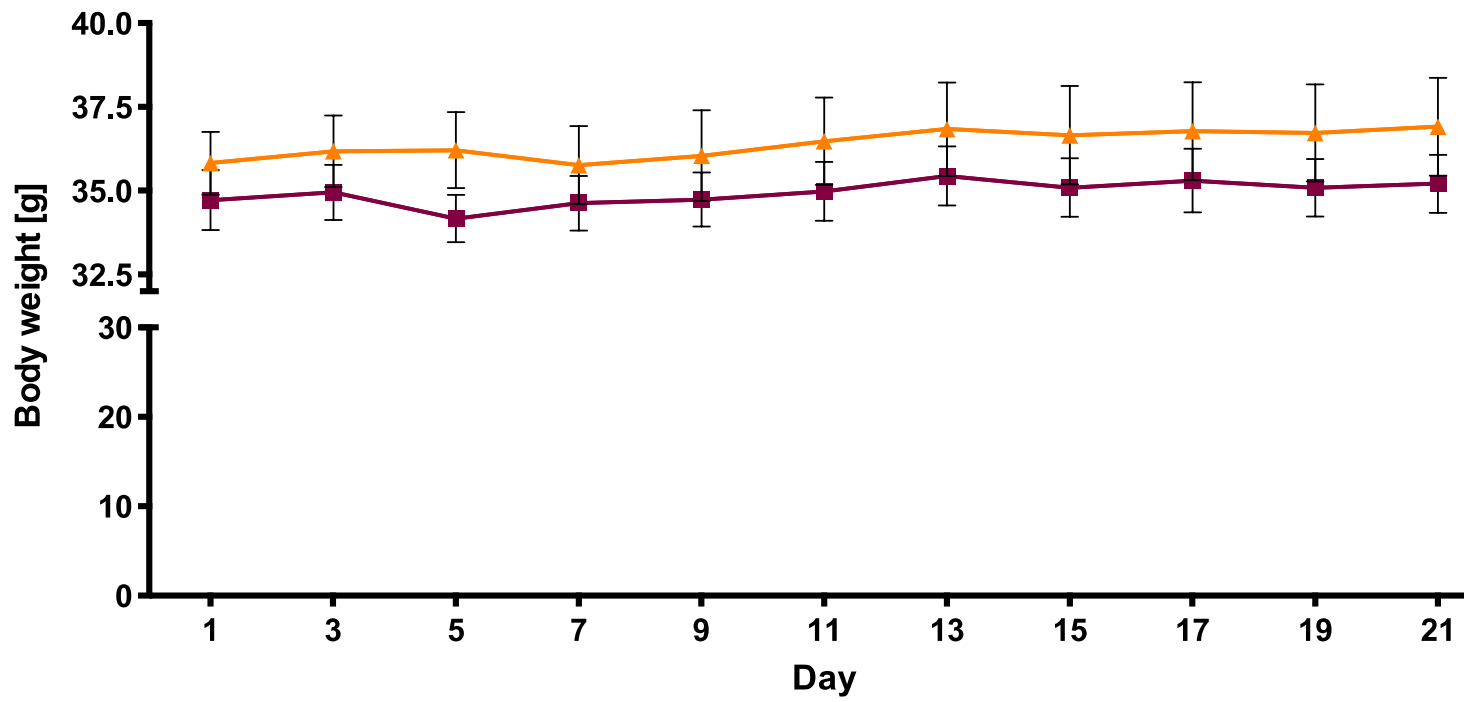

Figure 3-16: Chronic oral SAHA administration improves long-term object recognition. The Open field test did not reveal differences in exploratory behavior or anxiety (A). Novel object recognition memory was slightly improved by chronic SAHA treatment (B). Body weight did not change greatly over the 3-weeks feeding period(C). 


\subsubsection{Gene Expression in the Aged Hippocampus}

We next tested, whether we could detect transcriptional changes in the aging hippocampus that might be associated with altered HDAC activity and/or described and observed age-associated memory impairments. To this end, we performed RNA-seq and microarray-based comparisons of mRNA levels for three different aging groups. The use of multiple technical approaches yields additional information concerning the validity of these methods. In addition each technique offers different advantages. Microarrays are broadly used and highly standardized in terms of technical processing and bioinformatics assessment. RNA-seq on the other hand features a novel, unbiased approach that is not limited to known gene annotations, is more sensitive towards low abundant transcripts (depending on sequencing depth) and may be used to estimate differential splicing by estimating transcript abundance on the exon level (Sîrbu et al., 2012). qRT-PCR, as a third way to determine RNA levels, is unparalleled in sensitivity and specificity and remains to be the "gold standard" for expression analyses, often used to validate genome-wide approaches but limited by the experimenter's selection and sequence availability.

\subsubsection{RNA Sequencing and Microarray Technology Reveal Dramatic Changes in Gene Expression}

In the following experiment three technical approaches were used, comprising single-end (SE) RNAsequencing, paired-end (PE) RNA-sequencing and Agilent microarrays. Figure 3-17A shows a summary of the groups used with each approach. In all cases, 3-month-old mice served as reference. RNA-seq yielded 12 - 37 million single-end reads of 50 bp per sample and 14 - 20 million paired reads of $200 \mathrm{bp}(100+100 \mathrm{bp})$ (Figure 3-17B). Reads were of high quality, with an average Phred quality score of $>30$ across the full read length (Figure 3-9C). Alignment of reads to the mouse genome using Bowtie2 resulted very high alignment rates in all RNA-seq samples (see Table 2-9).

Interestingly, 2-dimensional principal component analysis (PCA) revealed that "age" was a highly likely explanation for a great fraction of the variation between samples across all three comparisons, microarray and RNA-seq likewise (Figure 3-17D). Expression-based sample distances confirmed this notion. Several samples within each group showed good correlation (Figure 3-17E). 
A

\begin{tabular}{|c|c|c|c|}
\hline \multirow{2}{*}{$\begin{array}{l}\text { Age } \\
\text { group }\end{array}$} & \multicolumn{2}{|c|}{ RNA-seg } & \multirow{2}{*}{ Array } \\
\hline & SE & PE & \\
\hline $\begin{array}{c}3 \\
\text { months }\end{array}$ & $n=5$ & $n=3$ & $n=4$ \\
\hline $\begin{array}{c}24 \\
\text { months }\end{array}$ & $n=6$ & - & \\
\hline $\begin{array}{c}\geq 28 \\
\text { months }\end{array}$ & - & $n=3$ & $n=4$ \\
\hline
\end{tabular}

B

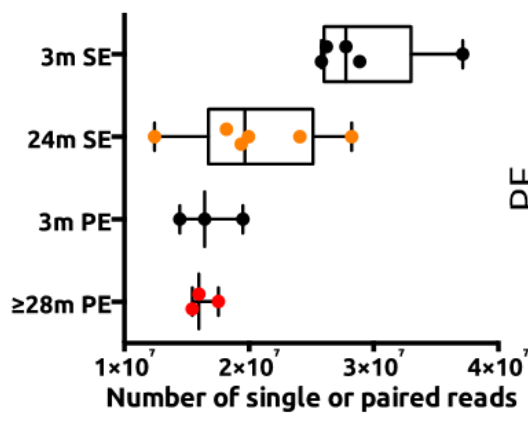

C

뜸

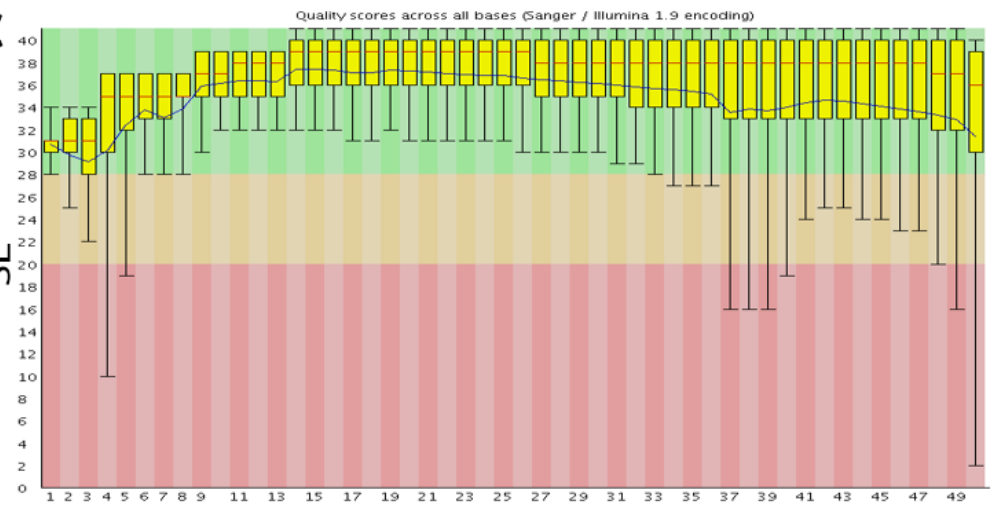

Quality scores across all bases (Sanger / Illumina 1.9 encoding)

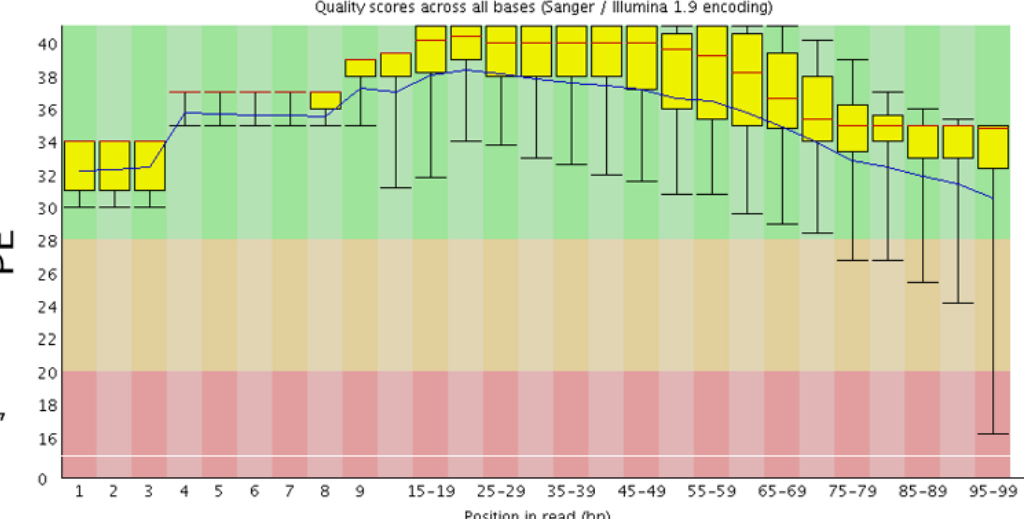

D
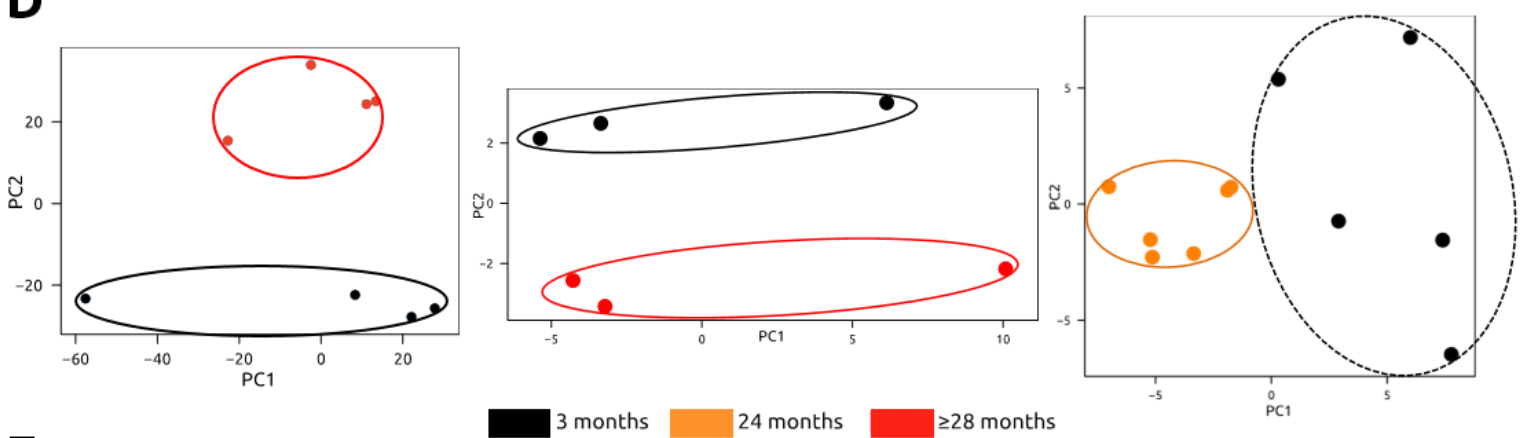

E
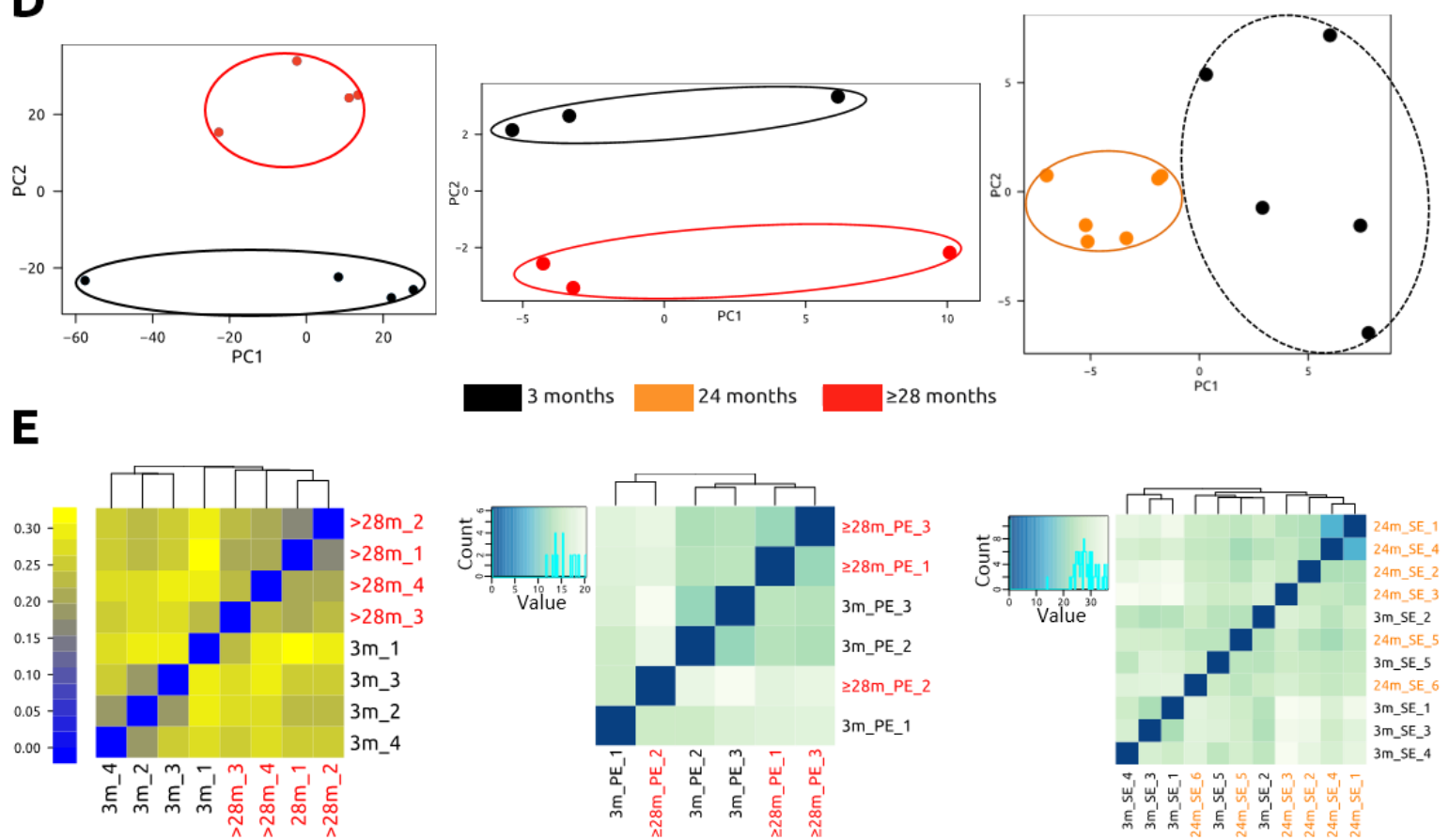

Figure 3-17: Expression analysis in the aging hippocampus: Group description and quality control. Overview of groups. For each column-wise comparison 3-month-old mice served as reference. The number of biological replicates is represented by $n(\mathbf{A})$. Median and range of total read counts for each group (B). Representative distribution of average Phred quality score across all reads of a sample dependent on the position within the read for single-end (SE) and paired-end (PE) libraries (C). 2-dimensional principal component analysis for each experiment. Scale is in \% (D). Sample distance and cluster dendrogram for all comparisons (E) 
We found robust changes of transcription in the aging hippocampus. In general, all approaches yielded similar results: In terms of the number of genes changing, we found comparable numbers in all comparisons, except for the number of downregulated genes in the PE RNA-seq comparison of 3 vs. $\geq 28$-month-old mice. In total, we found 841 genes that were significantly upregulated and 578 genes that were downregulated when comparing young and aged mice (Figure 3-18A). Among the upregulated genes, we found a substantial amount of overlap with comparable numbers between ages and methods, i.e. RNA-seq vs. microarray, both $3 \mathrm{~m}$ vs. $28 \mathrm{~m}$, gave a similar overlap as RNA-seq for $3 \mathrm{~m}$ vs. 24 vs. microarray (Figure 3-18B). Interestingly, overlaps among downregulated genes were considerably less pronounced (Figure 3-18C). Genes that were overlapping are given in Table 3-5, together with one possibility of how a particular overlap may be interpreted. We also found a negligible fraction of genes that were overlapping between up- and downregulated genes (Table 3-5).

Notably, we could not detect changes in HDAC expression, which would be related to decreased HDAC activity as described in 3.2.1.1. However, we found two HATs to be differentially regulated from 3 to $\geq 28$-month-old mice using the microarray data. These were Kat $2 b$ (a.k.a. Pcaf) upregulated with age) and Kat6b (a.k.a. Myst4, downregulated with age).

\begin{tabular}{|l|c|c|}
\hline \multicolumn{1}{|c|}{ Comparison } & $\frac{\text { Genes (Probes) }}{\text { upregulated }}$ & $\begin{array}{c}\text { Genes (Probes) } \\
\text { downregulated }\end{array}$ \\
\hline $3 \mathrm{~m}$ vs. $24 \mathrm{~m}$ RNA-seq & 376 & 239 \\
\hline $3 \mathrm{~m}$ vs. $\geq 28 \mathrm{~m}$ RNA-seq & 326 & 89 \\
\hline $3 \mathrm{~m}$ vs. $\geq 28 \mathrm{~m}$ Microarray & $374(423)$ & $284(330)$ \\
\hline Merged 3m vs. $\geq 24$ (nr) & 841 & 578 \\
\hline
\end{tabular}

B

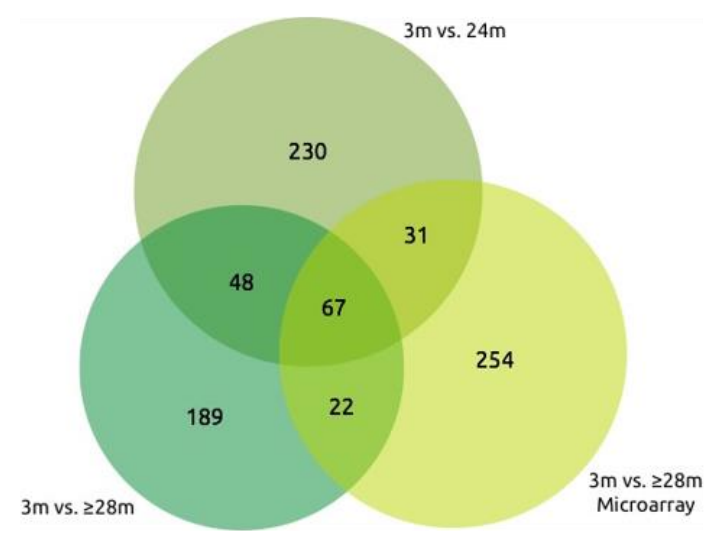

upregulated
C

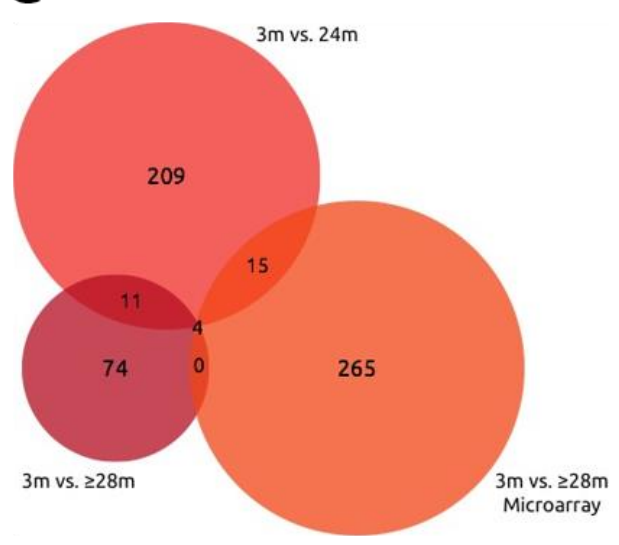

downregulated

Figure 3-18: Expression analysis in the aging hippocampus: DEGs. A comparable amount of genes was differentially regulated in the tree comparisons, with the exception the exception of downregulated genes in $3 \mathrm{~m}$ vs. $\geq 28 \mathrm{~m}$ RNA-seq. Merged lists are non-redundant (nr) lists of all genes found to be differentially regulated due to aging in general. Numbers of probes with significantly different signals (including redundant and nonannotated probes are given in brackets (A). Number of overlapping genes that are upregulated during aging, circle radius and intersection area correspond to value as closely as possible (B). Number of overlapping genes that are downregulated during aging, circle radius and intersection area correspond to value as closely as possible (C). 
Table 3-5: Overlapping DEGs between different comparisons. In the "Group" column also the technique and a possible interpretation of the particular group are given.

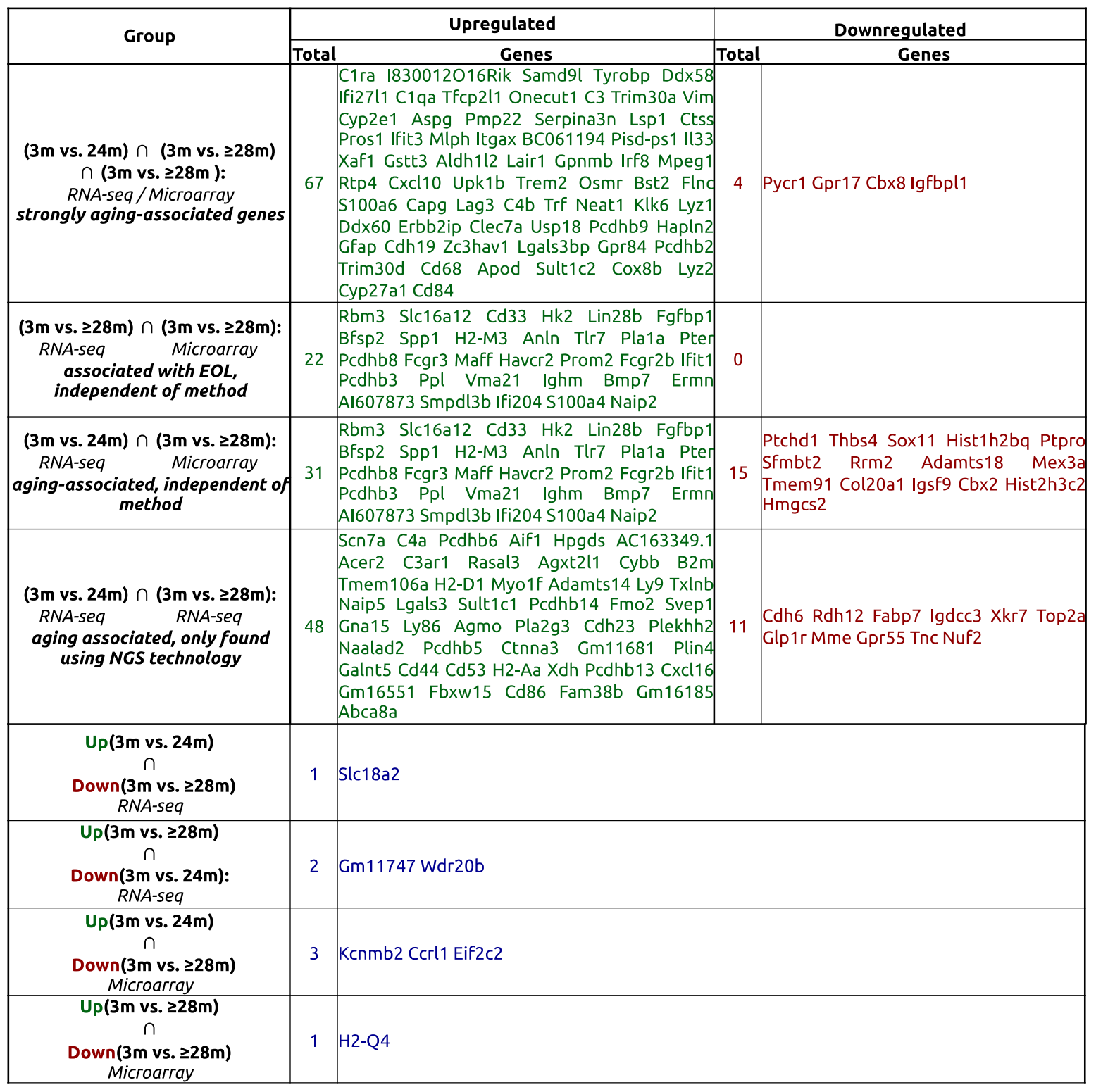

From a chromatin-biology point of view, another interesting observation was a considerable number of downregulated histone genes. To further examine, whether we could detect more functional groups of genes, we again performed GO-Term and KEGG-Pathway analysis as described in 2.6.3.

Within upregulated genes, all comparisons between young and aged showed great agreement. A substantial amount of genes was involved in a plethora of terms associated with biological processes of immune function and associated with MHC complexes (see Table 6-5 to Table 6-7). In addition many upregulated genes were associated with the plasma membrane. Molecular functions of upregulated genes included chemokine activity and calcium ion binding. Among the most enriched pathways were the complement-system cascade and other immune related pathways. Only a few functional categories distinguished groups. These included the regulation of RNA metabolic process (only seen among 3 vs. 24 -month-old mice), fatty acid biosynthesis (in $3 \mathrm{~m}$ vs. $\geq 28 \mathrm{~m}$, RNAseq), gland, bone and tissue development and regulation of apoptosis (in $3 \mathrm{~m}$ vs. $\geq 28 \mathrm{~m}$, microarray). 
Within downregulated genes, there were a lot less significantly enriched categories, which were also different between comparisons to a larger extend than categories of upregulated genes. This observation is in line with a smaller overlap among the downregulated genes. Categories that discriminated the groups included chromatin-related processes (only among 3 vs. 24-month-old mice and found with microarray), neurotransmitter receptor activity (in $3 \mathrm{~m}$ vs. $\geq 28 \mathrm{~m}$, RNA-seq) and mitosis (only found with microarray).

We also merged lists of all three comparisons between young and old to determine the general theme of up- and down-regulated processes associated with hippocampal aging. Though the lists were now considerably larger, and therefore more prone to random effects, analysis of these lists greatly confirmed findings resulting from analyses of individual comparisons and increased enrichment and statistical significance for several categories (Table 3-6). In particular, complementpathway enrichment among upregulated genes and chromatin-related categories among downregulated remained the highest ranked functional categories. Interestingly, neurogenesis and neuroactive ligand-receptor activation were terms, where enrichment was enhanced after merging lists of downregulated genes. 
Table 3-6: GO-Term and KEGG-Pathway analysis of merged, non-redundant lists for $3 \mathrm{~m}$ vs. $\geq 24 \mathrm{~m}$ (841 upregulated, 578 downregulated).

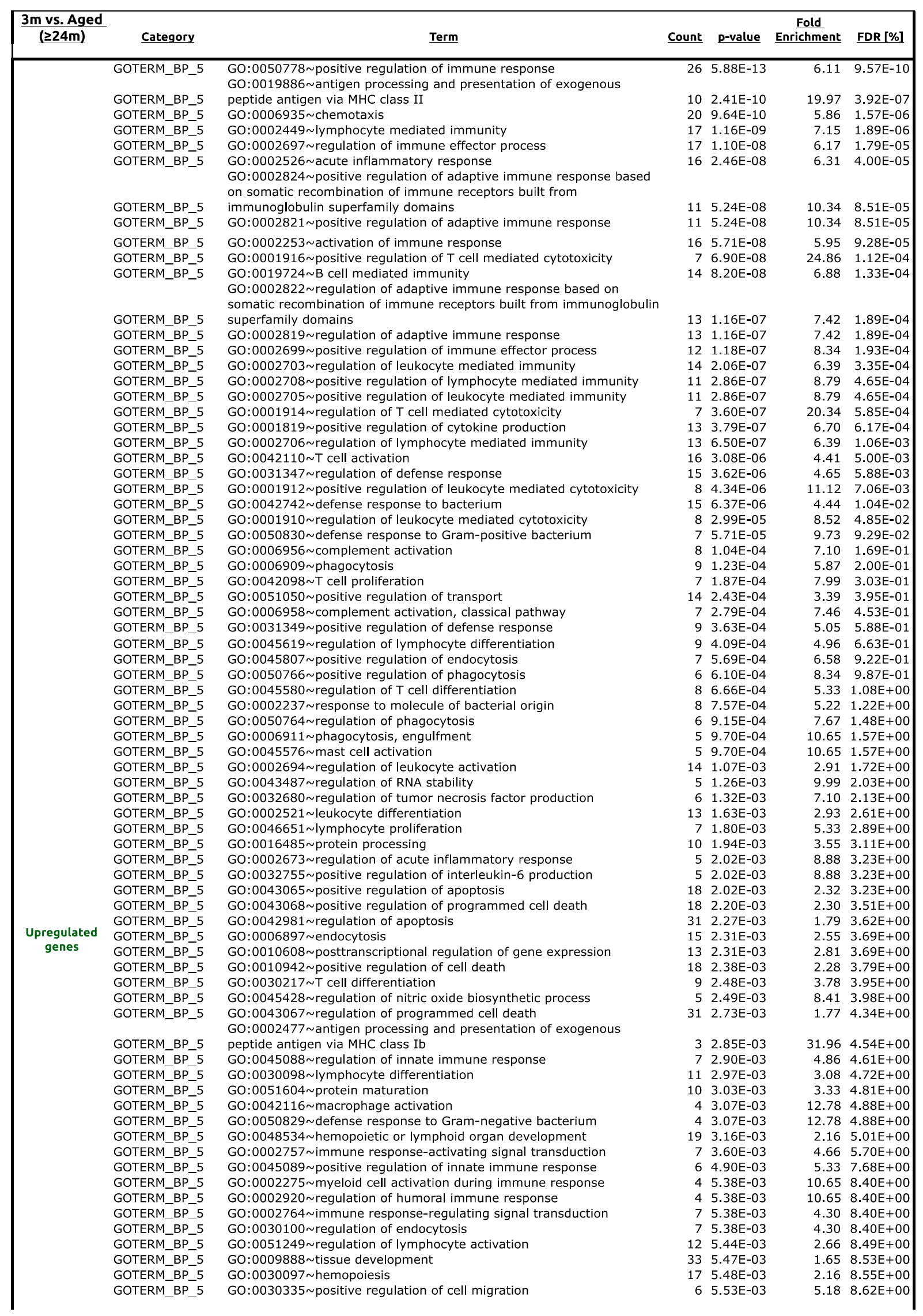


Table 3-6: (continued)

\begin{tabular}{|c|c|c|c|c|c|c|}
\hline & GOTERM_CC_5 & GO:0042611 MHC protein complex & 14 & $6.34 \mathrm{E}-09$ & 8.39 & $8.22 \mathrm{E}-06$ \\
\hline & GOTERM_CC_5 & GO:0009897 external side of plasma membrane & 26 & $1.04 \mathrm{E}-08$ & 3.94 & $1.35 \mathrm{E}-05$ \\
\hline & GOTERM_CC_5 & GO:0042613 MHC class II protein complex & 6 & $7.25 \mathrm{E}-06$ & 18.71 & $9.39 \mathrm{E}-03$ \\
\hline & GOTERM_CC_5 & GO:0044459 plasma membrane part & 82 & $3.50 \mathrm{E}-05$ & 1.57 & 4.53E-02 \\
\hline & GOTERM_CC_5 & GO:0042824 MHC class I peptide loading complex & 5 & $1.15 \mathrm{E}-04$ & 17.32 & $1.49 \mathrm{E}-01$ \\
\hline & GOTERM_CC_5 & GO:0042612 MHC class I protein complex & 8 & $3.35 \mathrm{E}-04$ & 5.94 & 4.34E-01 \\
\hline & GOTERM_CC_5 & GO:0042825 TAP complex & 4 & $1.04 \mathrm{E}-03$ & 17.82 & $1.33 E+00$ \\
\hline & GOTERM_MF_5 & GO:0008009 chemokine activity & 11 & $3.25 \mathrm{E}-07$ & 8.60 & $3.98 \mathrm{E}-04$ \\
\hline & GOTERM_MF_5 & GO:0042379 chemokine receptor binding & 11 & $4.24 \mathrm{E}-07$ & 8.38 & $5.19 \mathrm{E}-04$ \\
\hline & GOTERM_MF_5 & GO:0004896 cytokine receptor activity & 9 & 4.55E-04 & 4.86 & 5.55E-01 \\
\hline & GOTERM_MF_5 & GO:0005509 calcium ion binding & 47 & $6.14 \mathrm{E}-04$ & 1.66 & $7.49 \mathrm{E}-01$ \\
\hline & GOTERM_MF_5 & GO:0042288 MHC class I protein binding & 4 & $2.71 \mathrm{E}-03$ & 13.21 & $3.26 \mathrm{E}+00$ \\
\hline & KEGG_PATHWAY & mmu04610:Complement and coagulation cascades & 20 & $2.85 \mathrm{E}-11$ & 6.86 & $3.34 \mathrm{E}-08$ \\
\hline & KEGG_PATHWAY & mmu04060:Cytokine-cytokine receptor interaction & 31 & $1.10 \mathrm{E}-08$ & 3.27 & $1.29 \mathrm{E}-05$ \\
\hline & KEGG_PATHWAY & mmu04612:Antigen processing and presentation & 18 & 4.77E-08 & 5.09 & $5.60 \mathrm{E}-05$ \\
\hline & KEGG_PATHWAY & mmu05332: Graft-versus-host disease & 14 & $2.06 \mathrm{E}-07$ & 6.21 & $2.42 \mathrm{E}-04$ \\
\hline & KEGG_PATHWAY & mmu04940:Type I diabetes mellitus & 14 & $5.76 \mathrm{E}-07$ & 5.72 & 6.77E-04 \\
\hline & KEGG_PATHWAY & mmu05330:Allograft rejection & 13 & $1.54 \mathrm{E}-06$ & 5.77 & $1.81 \mathrm{E}-03$ \\
\hline & KEGG_PATHWAY & mmu04062: Chemokine signaling pathway & 23 & $1.55 \mathrm{E}-06$ & 3.25 & $1.82 \mathrm{E}-03$ \\
\hline & KEGG_PATHWAY & mmu05322: Systemic lupus erythematosus & 17 & $1.65 \mathrm{E}-06$ & 4.25 & $1.94 \mathrm{E}-03$ \\
\hline & KEGG_PATHWAY & mmu05416:Viral myocarditis & 16 & 2.49E-06 & 4.38 & $2.92 \mathrm{E}-03$ \\
\hline & KEGG_PATHWAY & mmu05320:Autoimmune thyroid disease & 13 & $1.64 \mathrm{E}-05$ & 4.65 & $1.93 \mathrm{E}-02$ \\
\hline & KEGG_PATHWAY & mmu04621:NOD-like receptor signaling pathway & 12 & $2.02 \mathrm{E}-05$ & 4.98 & 2.37E-02 \\
\hline & KEGG_PATHWAY & mmu04640:Hematopoietic cell lineage & 13 & $8.01 \mathrm{E}-05$ & 3.98 & $9.40 \mathrm{E}-02$ \\
\hline & KEGG_PATHWAY & mmu04514: Cell adhesion molecules (CAMs) & 18 & $8.16 \mathrm{E}-05$ & 3.01 & $9.58 \mathrm{E}-02$ \\
\hline & KEGG_PATHWAY & mmu05310:Asthma & 8 & $2.13 \mathrm{E}-04$ & 6.24 & $2.50 \mathrm{E}-01$ \\
\hline & KEGG_PATHWAY & mmu04620:Toll-like receptor signaling pathway & 13 & 3.93E-04 & 3.38 & $4.60 \mathrm{E}-01$ \\
\hline & KEGG_PATHWAY & mmu05340:Primary immunodeficiency & 7 & $2.30 \mathrm{E}-03$ & 5.00 & $2.67 E+00$ \\
\hline & KEGG_PATHWAY & mmu04622:RIG-I-like receptor signaling pathway & 9 & 4.42E-03 & 3.41 & $5.06 \mathrm{E}+00$ \\
\hline & KEGG_PATHWAY & mmu04630: Jak-STAT signaling pathway & 14 & $5.72 \mathrm{E}-03$ & 2.37 & $6.51 \mathrm{E}+00$ \\
\hline & KEGG_PATHWAY & mmu04650: Natural killer cell mediated cytotoxicity & 12 & $7.28 \mathrm{E}-03$ & 2.53 & $8.22 \mathrm{E}+00$ \\
\hline \multirow{33}{*}{$\begin{array}{c}\text { Downregulated } \\
\text { genes }\end{array}$} & GOTERM_BP_5 & GO:0006333 chromatin assembly or disassembly & 10 & $8.36 \mathrm{E}-04$ & 4.02 & $1.29 \mathrm{E}+00$ \\
\hline & GOTERM_BP_5 & GO:0000087 M phase of mitotic cell cycle & 13 & $1.61 \mathrm{E}-03$ & 2.94 & $2.47 E+00$ \\
\hline & GOTERM_BP_5 & GO:0022008 neurogenesis & 24 & $2.68 \mathrm{E}-03$ & 1.96 & $4.09 E+00$ \\
\hline & GOTERM_BP_5 & GO:0007067 mitosis & 12 & 4.13E-03 & 2.77 & $6.24 \mathrm{E}+00$ \\
\hline & GOTERM_BP_5 & GO:0048699 generation of neurons & 22 & 4.74E-03 & 1.94 & $7.12 \mathrm{E}+00$ \\
\hline & GOTERM_BP_5 & GO:0006334 nucleosome assembly & 7 & $6.23 \mathrm{E}-03$ & 4.20 & $9.26 \mathrm{E}+00$ \\
\hline & GOTERM_CC_5 & GO:0005694 chromosome & 24 & $1.06 \mathrm{E}-05$ & 2.88 & $1.34 \mathrm{E}-02$ \\
\hline & GOTERM_CC_5 & GO:0044427 chromosomal part & 20 & 7.99E-05 & 2.85 & $1.00 \mathrm{E}-01$ \\
\hline & GOTERM_CC_5 & GO:0000785 chromatin & 13 & $2.88 \mathrm{E}-04$ & 3.58 & $3.62 \mathrm{E}-01$ \\
\hline & GOTERM_CC_5 & GO:0005875 microtubule associated complex & 8 & $1.38 \mathrm{E}-03$ & 4.78 & $1.72 \mathrm{E}+00$ \\
\hline & GOTERM_CC_5 & GO:0043232 intracellular non-membrane-bounded organelle & 62 & $1.67 \mathrm{E}-03$ & 1.47 & $2.08 \mathrm{E}+00$ \\
\hline & GOTERM_CC_5 & GO:0000786 nucleosome & 7 & $2.34 \mathrm{E}-03$ & 5.12 & $2.91 \mathrm{E}+00$ \\
\hline & GOTERM_MF_5 & GO:0008528 peptide receptor activity, G-protein coupled & 12 & $3.14 \mathrm{E}-05$ & 4.89 & $3.68 \mathrm{E}-02$ \\
\hline & GOTERM_MF_5 & GO:0008188 neuropeptide receptor activity & 7 & $1.71 \mathrm{E}-04$ & 8.26 & $2.00 \mathrm{E}-01$ \\
\hline & GOTERM_MF_5 & GO:0005216 ion channel activity & 19 & 7.69E-04 & 2.44 & $8.97 \mathrm{E}-01$ \\
\hline & GOTERM_MF_5 & GO:0022838 substrate specific channel activity & 19 & $1.10 \mathrm{E}-03$ & 2.37 & $1.28 \mathrm{E}+00$ \\
\hline & GOTERM_MF_5 & GO:0022836 gated channel activity & 16 & $1.50 \mathrm{E}-03$ & 2.55 & $1.74 \mathrm{E}+00$ \\
\hline & GOTERM_MF_FAT & GO:0030594 neurotransmitter receptor activity & 12 & $3.94 \mathrm{E}-06$ & 6.09 & $5.61 \mathrm{E}-03$ \\
\hline & GOTERM_MF_FAT & GO:0042165 neurotransmitter binding & 12 & $3.94 \mathrm{E}-06$ & 6.09 & $5.61 \mathrm{E}-03$ \\
\hline & GOTERM_MF_FAT & GO:0008528 peptide receptor activity, G-protein coupled & 12 & $3.79 \mathrm{E}-05$ & 4.82 & $5.39 \mathrm{E}-02$ \\
\hline & GOTERM_MF_FAT & GO:0001653 peptide receptor activity & 12 & $3.79 \mathrm{E}-05$ & 4.82 & $5.39 \mathrm{E}-02$ \\
\hline & GOTERM_MF_FAT & GO:0008188 neuropeptide receptor activity & 7 & $1.89 \mathrm{E}-04$ & 8.13 & $2.69 \mathrm{E}-01$ \\
\hline & GOTERM_MF_FAT & GO:0042923 neuropeptide binding & 7 & $1.89 \mathrm{E}-04$ & 8.13 & 2.69E-01 \\
\hline & GOTERM_MF_FAT & GO:0042277 peptide binding & 13 & $2.76 \mathrm{E}-04$ & 3.59 & $3.92 \mathrm{E}-01$ \\
\hline & GOTERM_MF_FAT & GO:0005216 ion channel activity & 19 & $9.91 \mathrm{E}-04$ & 2.40 & $1.40 \mathrm{E}+00$ \\
\hline & GOTERM_MF_FAT & GO:0022838 substrate specific channel activity & 19 & $1.42 \mathrm{E}-03$ & 2.33 & $2.00 \mathrm{E}+00$ \\
\hline & GOTERM_MF_FAT & GO:0022803 passive transmembrane transporter activity & 19 & $1.63 \mathrm{E}-03$ & 2.30 & $2.29 \mathrm{E}+00$ \\
\hline & GOTERM_MF_FAT & GO:0015267 channel activity & 19 & 1.63E-03 & 2.30 & $2.29 E+00$ \\
\hline & GOTERM_MF_FAT & GO:0022836 gated channel activity & 16 & $1.84 \mathrm{E}-03$ & 2.51 & $2.59 E+00$ \\
\hline & GOTERM_MF_FAT & GO:0046873 metal ion transmembrane transporter activity & 16 & $2.49 \mathrm{E}-03$ & 2.44 & $3.48 \mathrm{E}+00$ \\
\hline & GOTERM_MF_FAT & GO:0005261 cation channel activity & 14 & $4.24 \mathrm{E}-03$ & 2.49 & $5.87 \mathrm{E}+00$ \\
\hline & KEGG_PATHWAY & mmu04080: Neuroactive ligand-receptor interaction & 17 & $2.52 \mathrm{E}-04$ & 2.84 & $2.78 \mathrm{E}-01$ \\
\hline & KEGG_PATHWAY & mmu04020:Calcium signaling pathway & 13 & $1.29 \mathrm{E}-03$ & 2.96 & $1.42 \mathrm{E}+00$ \\
\hline
\end{tabular}




\subsubsection{Upregulation of the Complement Component C4 Correlates with Age and is Associated with} Increased Intronic H3K9 Acetylation

Among the upregulated genes with the highest fold-change in all comparisons between young and aged hippocampi was the $C 4 b$ gene (Fold changes: RNA-seq: $3 \mathrm{~m}$ vs. $24 \mathrm{~m}: 4.3 \mathrm{x}, 3 \mathrm{~m}$ vs. $\geq 28 \mathrm{~m}: 7 \mathrm{x}$, microarray: $5 x$ ). This gene's product, called complement component 4 (C4), is a precursor protein that is further processed several times during the complement cascade to form the C4a, C4b and c4d proteins or polypeptides.

We confirmed upregulation of $C 4 b$ by $\mathbf{q R T}$-PCR identifying a significant increase in 24 - and $\geq 28$ month-old mice (1way ANOVA: $F_{(2,14)}=13, p<0.001$, Tukey's multiple comparisons test: $3 \mathrm{~m}$ vs. $24 \mathrm{~m}$ : $p=0.034,3 \mathrm{~m}$ vs. $\geq 28 \mathrm{~m}: \mathrm{p}<0.001,6.6$-fold increase) and a linear trend between age and expression level $\left(R^{2}=0.62, p<0.001\right.$, Figure 3-19A).

We performed genome-wide occupation experiments for H3K9ac (ChIP-seq) to investigate, whether transcriptional regulation during hippocampal aging is associated with changes in activation-related histone acetylation marks. Interestingly, we found increasing enrichment of H3K9ac the $C 4 b$ locus with increasing age. However, H3K9ac enrichment was not restricted to the promoter region of the $c 4 b$ gene but was dispersed over two additional hotspots within the two largest intronic regions of the gene. Strikingly, increasing enrichment of H3K9ac with age was focused at the first of these intronic hot spots (Figure 3-19B). This intronic region features a GA-rich, low-complexity region. To determine potential functional relevance, we performed bioinformatics analysis of the sequence of this region. Using the TFSearch tool, multiple MZF1-binding sites were found as well as a binding site for p300, a.k.a. histone acetyltransferase Kat3b. 

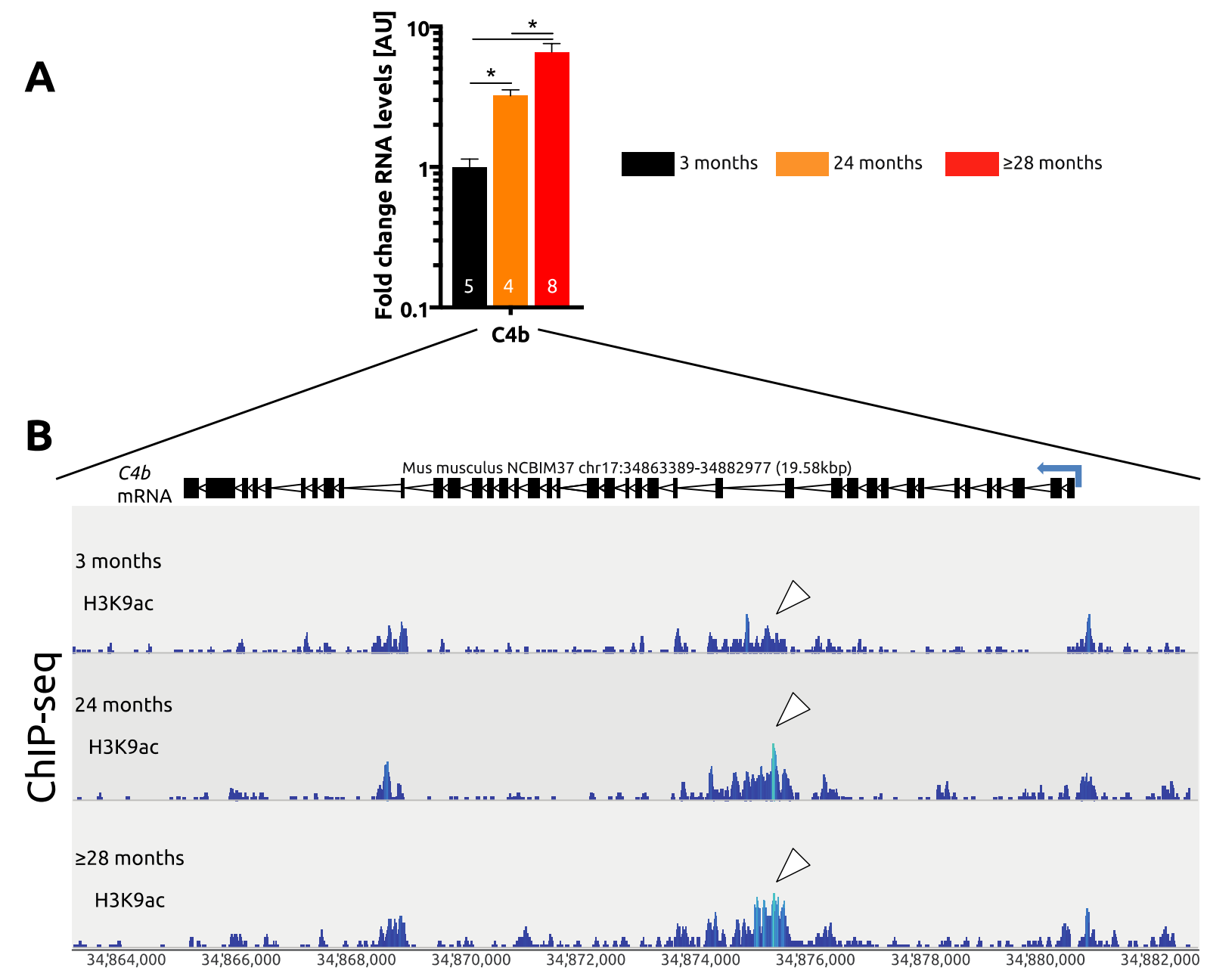

3 months
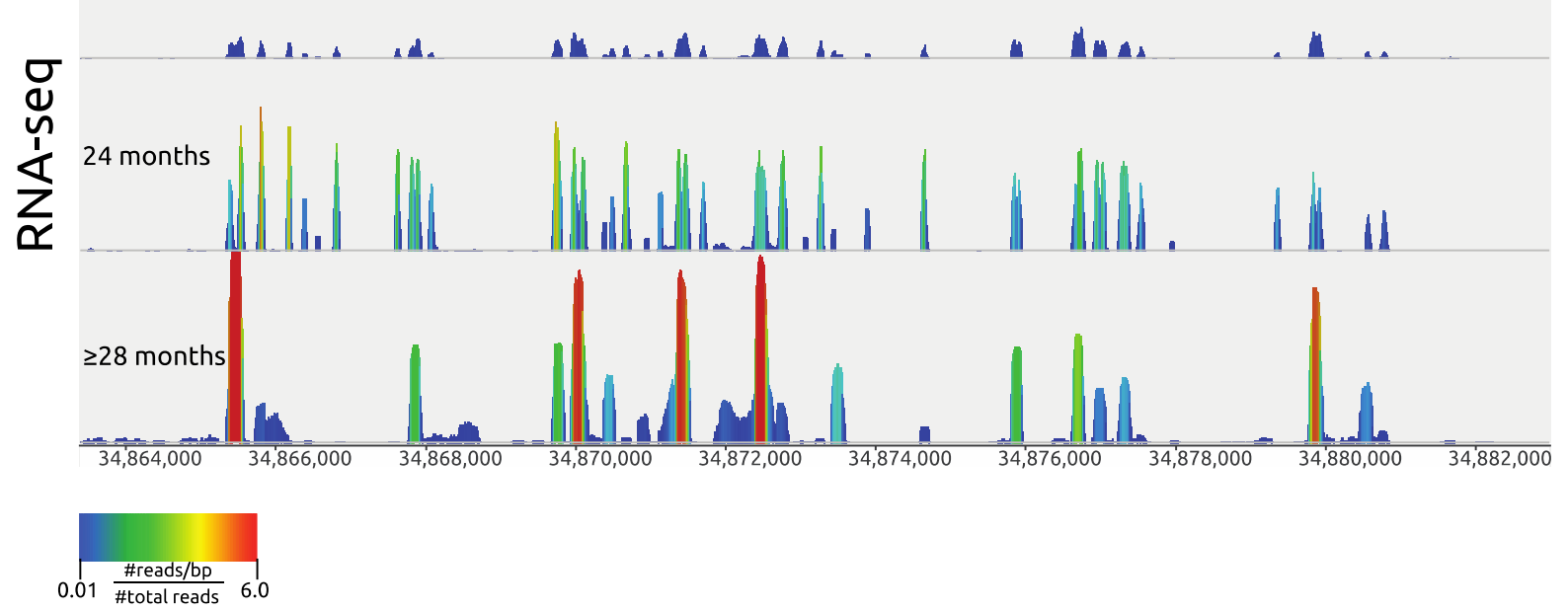

Figure 3-19: qRT-PCR validation and correlation with H3K9ac. qRT-PCR validation of $C 4 b$ upregulation during aging. Note the logarithmic scale. Normalized to $3 \mathrm{~m}$ group. Legend gives age in months. (A). Detailed view of the C4b locus. Data for enrichment of H3K9ac derived form ChIP-seq experiments (2 biological replicates, upper panel) correlate with increased $C 4 b$ mRNA abundance (arrowheads, B). Peak height and color correlate. 


\subsubsection{Increased C4 Protein Levels and Astrogliosis Accompany Hippocampal Aging}

For two of the most dramatically upregulated genes, namely $\mathbf{C} \boldsymbol{4 b}$ and $\mathbf{G f a p}$, we carried out immunohistochemical stainings to validate differential expression on the protein level and to explore the distribution of these upregulated proteins within the hippocampus.

Interestingly, fluorescent immunohistochemistry of aged brain tissue is handicapped by strong autofluorescent background (AFBG) that turned out to be aggregates of lipofuscin (Gray and Woulfe, 2005). Interestingly, lipofuscin aggregates were particularly larger and brighter in CA3 pyramidal cells compared to DG granule cells. The brightest aggregates, however, were found in microglia (see Figure 6-3)

For C4 stainings, we therefore produced overlay and subtraction images (using a different excitation wavelength) to discern true C4 staining from AFGB. We found C4 staining was almost absent in sections from 3-month-old mice, while there was prominent staining in sections from 24and $\geq 28$-month-old mice. Staining was found at filamentous structures or aggregates across the hippocampus and did not seem to localize inside cells (Figure 3-20A).

Using a Gfap specifc antibody, we found an increased number and density of astroglia in the hippocampus of aged mice that was evident across the whole hippocampus. This invasion of astroglia is also known as astrogliosis. In a more detailed magnification of the dentate gyrus, we could again see AFBG but Gfap staining was clearly distinguishable, since its localization pattern and the morphology of astroglia is easily recognizable. Upregulation on the RNA level was also confirmed by qRT-PCR, validating observed fold changes of RNA-seq and microarray (1.5-fold and 2fold, respectively; Figure 3-20B)

In conclusion, we could confirm upregulated expression for these two genes at the protein level. While Gfap is an astroglial marker and not known to be expressed by neurons, there is accumulating evidence that complement components, including $C 4 b$, are expressed in neurons and serve diverse functions in the brain (Davoust et al., 1999; Maier et al., 2008; Moriyama et al., 2011; Ramaglia et al., 2012; Schafer et al., 2012; Wyss-Coray and Rogers, 2012). Whether, however, upregulated C4 expression during aging is originating in glia cells or in neurons, we could not determine using immunohistological stainings. 
A

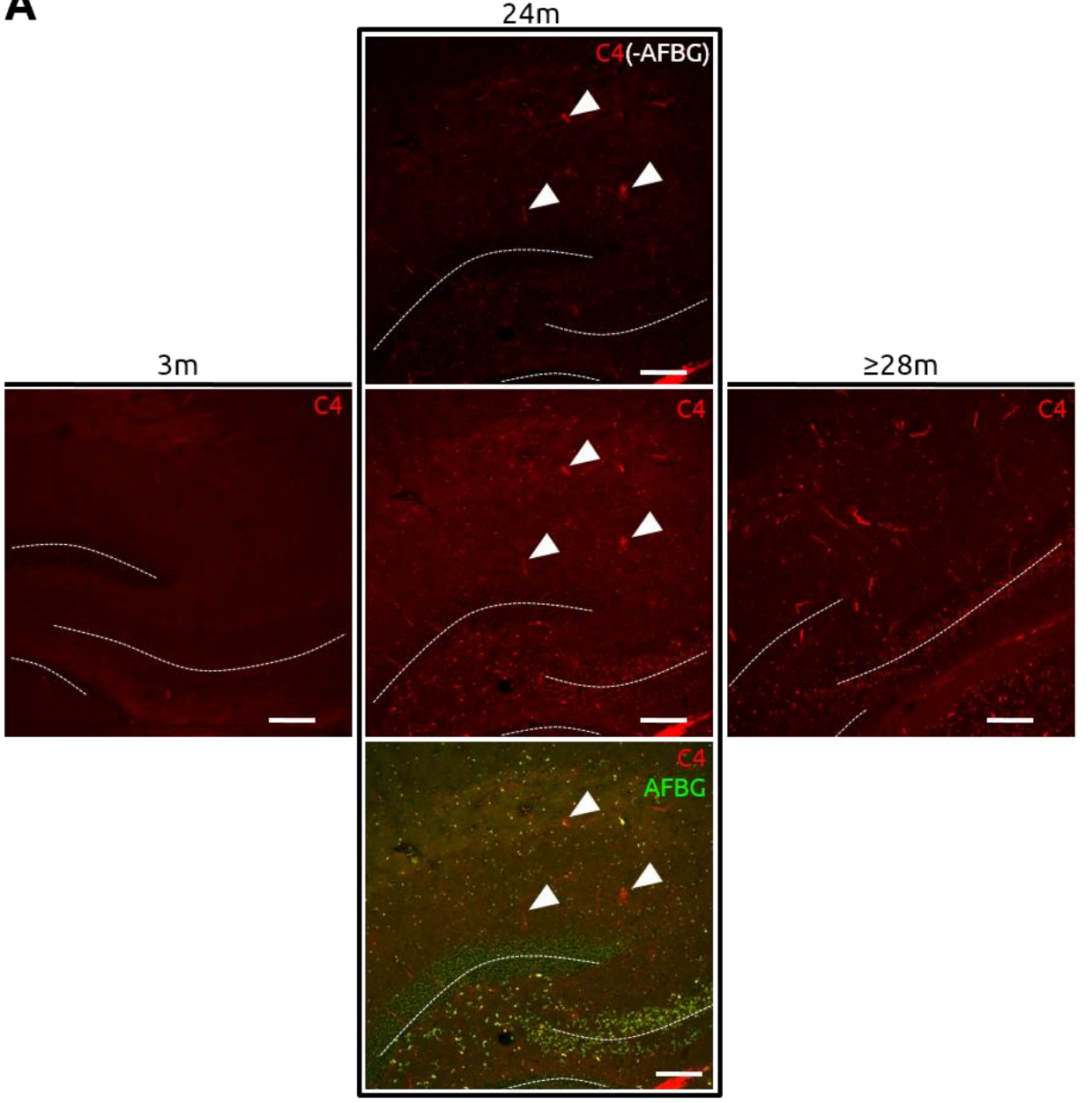

B
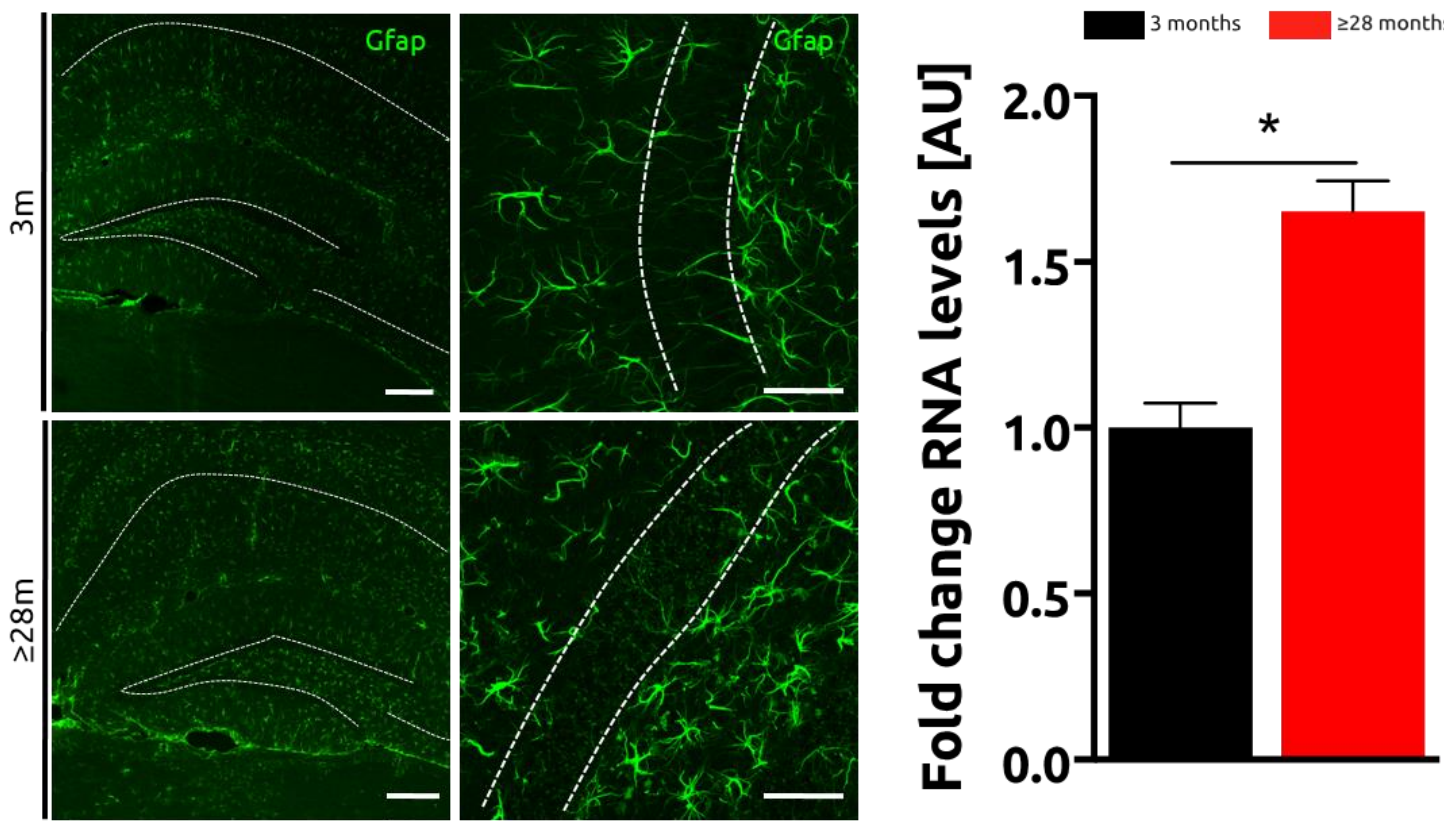

Figure 3-20: Increasing protein levels in the aging hippocampus. Staining of processed C4 protein and downstream products (C4, C4b, C4d) across tested ages. Strong autofluorescent background (Lipofuscin) complicated analysis and was subtracted $(24 \mathrm{~m}$, upper panel) or overlaid $(24 \mathrm{~m}$, lower panel) for demonstration purposes and to enhance true staining of C4 (arrowheads, A). Staining for astroglial marker Gfap shows increased number and density of Astroglia (Astrogliosis), which is in line with qRT-PCR validation of increase in mRNA level (B). White outlines show hippocampal neuronal cell layers (DG \& CA3). Representative images, scale bar is $100 \mu \mathrm{m}$ for $10 \mathrm{x}$ magnifications and $50 \mu \mathrm{m}$ for $63 \mathrm{x}$. 


\section{Discussion}

The data presented here contributes to our understanding of the role of Kat2a in transcriptiondependent memory formation and further deepens our knowledge about the transcriptional changes in the aging hippocampus and its association with histone acetylation.

Like the Results section, the Discussion section is divided into two parts that will help to review and interpret the data critically based on current literature. Some closing remarks integrate the results of this thesis into a larger context.

\subsection{Kat2a is an Important HAT in the Hippocampus that Regulates Long-Term Memory Formation}

\subsubsection{Kat2a conditional knockout mice exhibit several specific phenotypes related to memory function}

Kat2a, formerly known as Gcn5l2 in Mus musculus or Gcn5 across species, is a well-studied histone acetyltransferase. To my knowledge, the presented study provides detailed analysis of Kat2a function in the mammalian brain in the context of learning and memory for the first time.

Investigation of the expression pattern of Kat2a suggests an important role in hippocampal function. The expression of Kat2a mRNA was in agreement with IN SITU hybridization data retrieved from the Allen Brain Atlas, showing strong expression in pyramidal and granule cell layers of the hippocampal formation, especially in the CA region (Figure 3-1, Figure 6-1). On the protein level, we find Kat2a localized in the nucleus, with a very low concentration in cytosolic fractions, suggesting a major function in acetylation of histones and other nuclear proteins. Indeed, all known targets of enzymatic Kat2a-mediated acetylation are nuclear proteins, including several non-histone proteins (see 4.1.2).

We find high nuclear protein levels in cortical regions, higher even than in the CA1 and DG. Interestingly, CA3 protein levels of Kat2a were not different from cerebellum levels. Thus, the overall agreement of expression on the RNA and protein level is violated in certain brain regions. This may represent mechanisms of posttranscriptional regulation before or during translation (e.g. miRNA binding or positive/negative regulators of translational efficiency). However, these discrepancies may also have technical reasons as quantitative western blotting is not as sensitive as qRT-PCR in picking up differences (Bhuiyan et al., 2001). If we assume a correlation of expression level with functional relevance in a given cell type, these results support the notion that Kat2a plays an important role in the hippocampus and therefore memory-related functions in this brain area.

Use of the Cre-loxp-based conditional knockout system resulted in forebrain-specific knockout of Kat2a, demonstrated on DNA (Figure 2-1B), RNA (Figure 3-1A) and protein level, in the latter case using Western blot and immunohistochemical methodology. (Figure 3-1C,D, Figure 3-3A). However, a detectable amount of mRNA and protein (ca. 18\%) was remaining. This is likely to reflect Kat2a 
expression in inhibitory neurons and glia, as these cell types do not express the CamK2a-promoterdriven Cre recombinase. Together with the consideration of a glia:neuron ratio of about 2.5:1 in the rodent hippocampus (Bandeira et al., 2009), these low levels of residual expression suggest that Kat2a is mainly expressed in excitatory neurons.

An obvious phenotype of conditional knockout mice was a slight but significant reduction in body weight that was paralleled by a proportionally reduced brain weight. However, the ratio of brain weight to body weight remained unchanged. This strongly suggests that reduction in brain weight can be attributed to overall smaller body size. It can be hypothesized that reduced body size reflects the role of Kat2a in development and growth. However, as shown in several previous studies, CamKlla-driven, Cre-mediated recombination in our CamKIla-Cre line does not appear before postnatal day 19 and various studies, including studies from the host laboratory, have made use of this line in the context of learning and memory (Kerimoglu et al., 2013; Kuczera et al., 2011; Mayford et al., 1996; Minichiello et al., 1999; Rotenberg et al., 1996; Tsien et al., 1996). Even so, there might have been an effect on growth through hormonal signaling, as Kat2a is also deleted in regulatory parts of the endocrine system, e.g. the hypothalamus, upon CamKIla promoter activation. Indeed, a limited early hypothalamic activation of the CamKIla promoter is reported in a similar Cre line by the Jackson laboratory ${ }^{24}$ However, we did not observe overt neuronal loss, as seen by DAPI and NeuN (Rbfox3) staining (Figure 3-3B,C). Also the synaptic markers Map2, Syt and Synpr did not reveal changes in morphology (Figure 3-3C,D) and we there was no indication for differential expression of these genes in our RNAseq data. In addition, we looked at astrocytes and myelin distribution that may indicate indirect effects of Kat2a deletion in the neuronal environment. Again, we did not observe overt changes (Figure 3-4). Together, these findings argue for a moderate effect on growth in Kat2 $a^{\mathrm{f} / \mathrm{F} \text { tg }}$ mice that, however, seems to have no effect on normal brain function.

When we investigated the effect of Kat2a deletion on hippocampal baseline histone acetylation levels, we found H4K12ac levels to be increased, while H3K9ac and H4K14ac were unchanged (Figure 3-10). Since all three sites are suggested target sites for Kat2a catalytic activity, these results came as a surprise. While it is plausible that those sites may not change in baseline condition on the bulk level because of compensatory activity of other HATs in substitution for Kat2a, upregulation of $\mathrm{H} 4 \mathrm{~K} 12 \mathrm{ac}$ in the knockout condition is more complicated. It might reflect "overcompensation" in residual HAT activity or increased binding to genomic regions by other HATs. We did not find any evidence for increased, compensatory expression of acetyltransferases, however this does not exclude differential enzyme activity (see section 4.1.2 below). Other explanations include, that proper function of HATs generally limits acetylation to relevant genomic regions and boundaries (e.g. euchromatin), and an imbalance in HAT function leads to uncontrolled acetylation of regions that would normally not be acetylated. This might, in part, be the result of binding to now free binding sites that are normally occupied by Kat2a (either acetylated lysine residues for binding by bromodomains or genomic sequences) However, as there are no indications in the literature so far, this is purely speculative. Further investigation of genome-wide H4K12ac

\footnotetext{
${ }^{24}$ The provided information can be found on the webpage for the mouse line B6.Cg-Tg(Camk2a-Cre)T29-1Stl/J. The data in question is, however, not provided in a citable format ("data not shown"), so that it remains an unproven claim.
} 
distribution would be necessary to determine which genomic regions are targeted by this increase. Finally, also a non-cell-autonomous effect cannot be excluded. In summary, these data emphasize that IN VIVO histone acetylation is a tightly regulated, highly dynamic and complex process that is influenced by a plethora of factors, including HAT-HDAC balance, upstream and downstream effects of the transcriptional machinery and not at least the metabolic status of the cell. Future experiments should be directed to the investigation of stimulus-dependent histone acetylation.

The behavioral phenotype of Kat2 $a^{f / f ; t g}$ mice however met with expectations. We did not observe an effect of Kat2a deficiency on exploratory behavior or basal anxiety, as tested by the open field and the elevated plus maze paradigms (Figure 3-5A,C). In the cross maze as well as in the short-term novel object recognition paradigm, both groups performed better than chance level, demonstrating intact spatial working memory (Figure 3-5D) and short-term memory (Figure 3-5E), respectively. We did not observe differences between conditional knockout and control mice in the Rotarod performance test. This test involves testing for physical strength as well as motor learning, and is dependent on proper cerebellum function (Coughenour et al., 1977; Goddyn et al., 2006; Shiotsuki et al., 2010), which was similar in both groups as indicated by an increase in latency to fall over time (Figure 3-5B). Given, that all aspects of these behavioral control parameters were normal, there is no reason to assume, that the growth effect (see above) leads to functional impairments.

We found a specific impairment of long-term memory as shown by decreased object recognition, and impaired performance in contextual fear conditioning ( $\mathrm{CFC}$ ) and Morris' water maze (MWM) (Figure 3-5E,F, Figure 3-6). While the effect on object-recognition and aversive memory was relatively mild, the spatial memory impairment was prominent. The Kat2 $a^{f / f ; t g}$ demonstrated a slower decrease in escape latency during the training and showed no preference for the platform region in the probe test (Figure 3-6A,C). The parameters of platform crossings and time spent over the former platform region during probe test also supported this finding, though here the differences were not statistically significant (Figure 3-6D,E). During the training phase, 2 way ANOVA did not reveal a significant interaction between genotype and training day, indicating a similar negative slope for escape latencies, with a shifted starting point. Indeed, differences were already observed on the first day. This fact may be attributed to the protocol we used, considering that four individual trials at each training day allow for within-day improvement of performance. A visual form of MWM with an independent group of mice was used to rule out possible effects of the gene knockout on vision. Both groups showed a very low escape latency on day 1 already and further decreasing latencies during the following days, thereby excluding visual impairment. Yet, we observed a higher latency in Kat2 $a^{f / f ; t g}$ mice on day 2 (Figure 3-6A). This result may as well be a sign of spatial learning impairment, since the visual task also involves learning to navigate in the pool and to recognize that the platform is the only way out of the maze.

Contextual fear conditioning as well as spatial navigation in the watermaze clearly depend on proper hippocampal function (Anagnostaras et al., 2001). Conversely, the role of the hippocampus in novel-object-recognition memory is controversial based on the literature (Barker and Warburton, 2011). As object recognition is a rather broad term, the brain region dependency seems to change with the specific protocol used for testing. According to a systematic study in rats by Barker and 
Warburton, object preference, as tested in the present thesis, does not involve the hippocampus but rather the perirhinal cortex (Barker and Warburton, 2011). Experiments by Wood and colleagues support this notion (Haettig et al., 2011; Stefanko et al., 2009). Though, in our paradigm, mice with forebrain-wide neuronal Kat2a deficiency performed slightly worse compared to controls, they showed a strong preference for the novel object, indicating specificity of Kat2a function for hippocampus-dependent memories.

Taken together, these data suggest, that Kat2a plays a major role in hippocampus-dependent longterm memory and supports the possibility that Kat2a loss-of-function also affects memory functions depending on other brain regions. In order to exclude any effects on development and to investigate purely hippocampus-dependent functions, future experiments would need to aim for a more focal loss-of-function approach, such as making use of AAV-mediated Cre expression under a neuron-specific promoter.

Interestingly, phenotypic evidence for a role of Kat2a in memory-related function is paralleled by data from electrophysiological measurements at the CA3-CA1 synapse (Schaffer collaterals). Long-term potentiation is claimed to be a molecular correlate of the memory trace, since strong stimulation can have detectable after-effects lasting for up to several hours. We found severe impairment in long-term potentiation in Kat2 $a^{\mathrm{f} / \mathrm{f} \text {; } \mathrm{tg}}$ mice. While input/output correlation, pairedpulse inhibition/facilitation and baseline recording during LTP measurement were comparable, tetanization in the LTP protocol did result in a more modest potentiation and faster decay thereof, returning to baseline within the measurement time window. This indicates a genotype effect during the early phases of LTP with increasing severity in later stages. Both alterations may be explained by Kat2a function in transcriptional regulation: Kat2a may on one hand regulate a set of genes that is involved in or modulates post-synaptic function, like neurotransmitter receptors. On the other hand, the fact that later phases of LTP are also affected is likely explained by Kat2a playing a role in stimulus-dependent regulation of genes necessary for mRNA and protein-synthesis-dependent forms of LTP. Our data however, do not exclude the possibility that Kat2a acetylates non-histone proteins during any of the steps involved in LTP establishment and maintenance.

\subsubsection{Stimulus-induced gene-expression}

To find possible molecular mechanisms for the observed phenotypes and potential target genes of Kat2a we performed transcription profiling of the complete CA1 mRNAome in a stimulusdependent manner, using a novelty-exposure paradigm (Figure 3-8) with subsequent, regionspecific transcriptome sequencing. RNA-seq is a powerful application of next-generation sequencing technology. One of its advantages is the possibility to visualize individual transcripts. Interestingly, we could also visualize the transcript resulting from recombination-mediated deletion of multiple exons and introns within in the Kat2a gene locus, thereby confirming the proposed knockout mechanism and the genotype of the mice used for the experiment (Figure 3-9F). The observed higher read count in Kat2a exons 1, 2, and the most-3' exon may reflect an attempt to compensate for absent Kat2a by upregulation of Kat2a expression, since transcription only leads to 
non-functional RNA. We can however only speculate about whether this transcript is processed further or even translated.

Principal component and sample distance analysis revealed that profiles of mice with different genotypes were not as clearly differentiated as mice with different treatment (home cage or novelty-exposed, Figure 3-9D,E). However, there were robust changes in gene expression between groups when compared pairwise (Figure 3-9G,H).

By comparing expression of group $\boldsymbol{A}$ vs. $\boldsymbol{B}$, we could determine the effectiveness of our novelty protocol. Novelty exposure induced the expression of several activity-related genes required for transcriptional activation (e.g. Fos, the Egr's and the Nr4a's) as well as neuronal immediate early genes (IEGs) involved in dendritic function like Homer1. GO-Term and KEGG pathway mining confirmed this observation, revealing enriched terms related to positive regulation of transcription and the MAPK-signaling pathway, that is known to play a role in neuronal activity, plasticity and memory (Table 6-1, Sweatt, 2001). We only found half as many downregulated genes, which did not reveal overall significant enrichment in specific functional categories except for 4 genes that are generally involved in regulation of proliferation or growth (Egf, Gdf3, Fgf22 and Gli2). This observation is in line with the expectation that novelty exposure mainly stimulates certain transcriptional programs rather than inhibiting a particular group of genes.

To determine which genes change expression due to Kat deficiency in young, healthy, naïve mice, we needed to compare groups $\boldsymbol{A}$ and $\boldsymbol{C}$. Here, we found a total of 104 DEGs (68 up, 36 downregulated, including the Kat2a gene $)^{25}$. These genes revealed only weak enrichment of functional categories. However, a relatively large number of upregulated genes were associated with the plasma membrane, suggesting a role in communication with the outside environment, e.g. cell-to-cell communication (Table 6-2). Additionally, we could determine some genes associated with neuronal signaling, as represented by terms associated with activation of phospholipase $C$ (PLC) and the neuroactive ligand-receptor interaction pathway. These genes were the serotonin receptors Htr2a and Htr2c, the neuropeptide receptor Npbwr1 and the hypocretin receptor Hcrtr2 (discussed below).

The $\boldsymbol{B}$ vs. $\boldsymbol{D}$ comparison is the most informative comparison when analyzing stimulus-induced gene expression that is potentially regulated by Kat2a. In this respect, differences in the comparisons of $A$ vs. $B$ and $C$ vs. $D$ also provide insight, since genes that are only novelty-regulated in the control condition but not in conditional knockout mice are likely to be dependent on Kat2a function (Table 3-4). However, the latter is less informative since statistical considerations need to be taken into account. For example, a gene might be below the threshold for statistically significant DEGs in comparison $C$ vs. $D$ (C: $\mathrm{n}=6, \mathrm{D}: \mathrm{n}=7$ ) but not in comparison $A$ vs. $B(\mathrm{~A}: \mathrm{n}=6, \mathrm{~B}: \mathrm{n}=5)$, though it had a similar average fold change in both comparisons. Indeed, this was the case for the IEG ArC, which was significantly upregulated in conditional knockout mice upon novelty exposure, but not in control mice, because it failed to reach statistical significance in the latter ( $p_{\text {adj }}=0.22$ ). However, when comparing $B$ vs. $D$ directly, there was - as expected - no difference in Arc expression.

\footnotetext{
${ }^{25}$ For a full list, see Supplementary Table in Appendix 6.2.1
} 
Nevertheless, this comparison showed the highest number of DEGs, with respect to up- as well as downregulated genes, suggesting that Kat2a has a larger effect on transcription when neurons become activated. Interestingly, comparisons $A$ vs. $C$ and $B$ vs. $D$ shared only a minority of DEGs (28 upregulated, 12 downregulated, including Kat2a itself, Figure 3-9G,H and Table 3-4) further supporting this hypothesis. Among upregulated genes in comparison $B$ vs. $D$ we found genes that are associated with calcium ion-binding and extracellular-matrix interaction (Table 6-3). These categories certainly are relevant for proper neuronal function and signaling. One of the calcium binding genes is Ano2 (a.k.a. anoctamin2 or TMEM16B), which is a calcium-activated chloride channel. This channel is used for repolarization during an action potential as shown by knockdown and pharmacological inhibition in hippocampal neurons (Huang et al., 2012). Thus, upregulation of this gene may modulate action potentials towards shorter spike durations without affecting EPSP amplitude or slope (Huang et al., 2012). Ano2 was also found upregulated in comparison $A$ vs. $C$ and if upregulation correlates with shorter duration of action potentials and thus decreased time for signal integration this provides a possible explanation for the observed electrophysiological phenotype of Kat2 $a^{f / f i t g}$ mice (i.e. impaired LTP while input/output function was normal).

Interestingly, several downregulated genes were associated with neuronal activities, including serotonin receptor activity, and translation (i.e. ribosome components). Downregulation of translation-associated genes may be a sign of impaired DE NOVO protein synthesis, which is implicated with LTP and LTM as described in 1.1.3.1. The downregulated serotonin receptors only partly overlap with those found in the $A$ vs. C comparison (Htr2a), the additionally regulated 5-HT receptors are Htr1a and Htr1b. In total, there are 4 out of the 16 known 5-HT receptors encoded in the mouse genome (10 of those expressed in CA1, Andrade, 1998) downregulated in Kat2 $a^{\mathrm{f} / \mathrm{i} \text { tg }}$ mice (Htr1a/b and Htr2a/c). Indeed, novelty-related behavior has been linked to the serotonin system (Kerman et al., 2011). Furthermore, serotonin receptors are well known to play a role in learning and memory (Bert et al., 2008; King et al., 2008; Ogren et al., 2008). In particular, Htr1a and Htr2a were found to modulate synaptic activity IN VITRO and IN VIVO (Arvanov and Wang, 1998; Kojima et al., 2003; Mori et al., 2001).

Together, these results provide a potential regulatory mechanism relating Kat2a deficiency to reduced LTP and impairments in long-term memory consolidation. Alterations of neuronal excitability may result from differential serotonergic modulation and calcium sensing in resting as well as active CA1 neurons.

By comparing groups $\boldsymbol{C}$ and $\boldsymbol{D}$ one can most clearly see which genes are regulated by noveltyexposure independently of Kat2a. Interestingly, we found similar results as in the $A$ vs. $B$ comparison in terms of IEGs. This is in line with previous reports of IGE expression in Cbp-loss-of-function (Valor et al., 2011; Wood et al., 2006) and HDACi-treated (Vecsey et al., 2007) mice after cFC. Here, we also found enrichment of transcription associated terms among upregulated genes, including the MAPKsignaling pathway and no evidence for overrepresented functional categories within downregulated genes. Therefore it is not surprising, that a considerable fraction of upregulated genes are shared between $C$ vs. $D$ and $A$ vs. B. However, an equally large fraction was not overlapping with $A$ vs. $B$, indicating possible compensatory upregulation. Again, there might also be 
a few genes that are different due to statistical issues (see above). We could not identify any functional pattern among the genes only upregulated in $C$ vs. D. Remarkably, almost no downregulated genes in the two novelty comparisons overlap. This supports the earlier notion that novelty, and in turn neuronal activity, induces active gene transcription rather than downregulates a certain set of genes.

\section{What are potential Kat2a target genes in the CA1?}

Being a protein with enzymatic activity towards other proteins as well as a transcriptional activator Kat2a has two kinds of potential target genes. The first kind are targets of lysine acetylation, such as specific lysine residues in the histone tail domains as well as non-histone proteins. In fact, several non-histone proteins are described to be acetylated by Kat2a/Gcn5, including the transcriptional regulators p53, Myc and Pcg1a (Patel et al., 2004). The capability of Kat2a to autoacetylate itself is, in contrast to autoacetylating Kat2b, controversial (Herrera et al., 1997; Vernarecci et al., 2010).

The second kind of Kat2a targets are genes that are activated by Kat2a-mediated acetylation of histones within the loci of these genes. To date, it is still not clear whether each HAT regulates a different set of genes or whether histone acetylation is a non-specific feature of transcriptional activation in general. In the latter case, target genes may vary between organisms, cell types and even treatments or stimuli. HATs are said to be "co-activators", which expresses that they aid in transcriptional activation but do not take the lead, i.e. they are not selective for specific genes and this function needs to be accomplished by other sequence specific DNA binding proteins (e.g. classical transcription factors) or - possibly - (non-coding)RNAs (Vernarecci et al., 2010). In conclusion we cannot speak of target genes in general but rather of target genes in a particular setting or situation, which in this case is the CA1 region in the basal state and upon novelty exposure. Interestingly, the notion that different situations or settings require different sets of genes to be regulated by Kat2a is supported by a related study in Kat2a conditional knock mice using a nestin-Cre-based knockout approach. Neither did the differentially regulated genes in knockout neural stem and precursor cells show substantial overlap with genes found in this study nor did the enriched functional categories (Martínez-Cerdeño et al., 2012). This further supports specificity of Kat2a function the regulation of genetic programs in the adult hippocampus.

To identify potential target genes for Kat2a in this setting, a simple comparison between young, naïve controls and CKO mice ( $A$ and $C$, respectively) can only give a static impression on the transcriptional profile of CA1 neurons in basal state because the true effect of knockout-mediated deregulation may be masked by many other mechanisms that compensate for Kat2a loss-offunction. In order to identify dynamic changes in response to Kat2a deletion, we designed the novelty exposure paradigm. As mentioned in Table 3-5, genes that are regulated by novelty exposure in control mice, but not in Kat2 $a^{\mathrm{f} / \text {; tg }}$ mice, are likely to be target genes of Kat2a regulation in this particular situation ( $A$ vs. $B$ only). In particular, the intersection of upregulated genes in $A$ vs. $B$ and downregulated genes in $B$ vs. $D$ deserves special attention. These genes are Vamp5, Hmgb2, Mc4r and Fam83d. Vamp5, a.k.a. myobrevin, is a plasma membrane associated protein, important for vesicle fusion (Zeng et al., 1998, 2003). Though its localization has not been studied in neurons, it has been shown to be upregulated by LTP induction and electroconvulsive therapy in rats (Elfving et 
al., 2008; Romcy-Pereira et al., 2009). Thus, this gene may be regulated by neuronal activity and is possibly involved in neurotransmitter release or other cell communication processes. Hmgb2 is a member of the high mobility group proteins, which are non-histone components of chromatin involved in regulation of transcription and DNA bending (Thomas, 2001). Therefore, this gene may be involved in stimulus-dependent transcription and nuclear architecture. Interestingly, the $M c 4 r$ gene has been implicated in learning and memory and synaptic plasticity before (Cui et al., 2012; Shen et al., 2013). The gene encodes a melanocortin receptor that is also known to regulate feeding behavior (Cui et al., 2012). Fam83d is a protein associated to the mitotic spindle apparatus in dividing cells (Varisli, 2012). About a role in postmitotic neurons however, possibly in microtubuleassociated trafficking, I can only speculate. Together, these genes may very well belong to a larger transcriptional program activated by neuronal activity that is, at least in part, likely to be regulated by Kat2a-mediated histone acetylation.

In summary, we report here for the first time a comprehensive view of changes in the transcriptome of the murine hippocampal CA1 region after novelty exposure and present evidence for a role of Kat2a in stimulus-dependent regulation of neuronal gene expression. Generally, this is also the first description of HAT-dependent, stimulus-induced gene expression IN VIVO that goes beyond selection of single target genes. However, I am aware that the high number of upregulated IEGs in both genotypes indicates that the second wave of induced, long-term-plasticity-related transcription (see 1.3.2 and Figure 1-1) is yet to come and may be regulated by Kat2a to an even larger extent. This could easily be determined experimentally by investigating the transcriptome at a later time point after novelty exposure (e.g. in the range of several hours).

In our setting, we could not find evidence for a crucial role of Kat2a in regulation of IEGs. This observation is in contrast to the fact that regulation of IEGs and stress-induced genes by Kat2a, or promoter occupancy of these by Kat2a-containing complexes, has been described in multiple situation and organisms, ranging from salt stress in yeast and drosophila to induction of primary response genes in mammalian cells of the immune system. (Hargreaves et al., 2009; Krebs et al., 2011; Nagy and Tora, 2007; Nagy et al., 2010; Spedale et al., 2012).

Kat2a is a learning-inducible gene itself. It was found upregulated one hour after a fear-conditioning stimulus (Peleg et al., 2010). In our novelty-exposure paradigm however, we did not find any HATs differentially regulated, neither in response to novelty exposure nor to Kat2a deficiency. Upregulation of HATs in the latter would have indicated a possible compensatory effect. Especially, the closely related HAT Kat2b, which has been implicated in learning and memory before (Bousiges et al., 2010; Gaub et al., 2010; Maurice et al., 2008), did not show upregulation. Together, these findings argue for a non-redundant and rather specific function of Kat2a. But it also reveals that a simple novelty exposure paradigm can be differentiated from a memory-inducing paradigm on the level of the transcriptome. While there may be overlapping molecular and cellular mechanisms, a further experimental dissection of the different settings is likely to reveal a distinct expression profile in, for example, an associative learning paradigm like fear conditioning, and may as well depend on Kat2a regulation (Peleg et al., 2010). 


\subsubsection{A dose dependent effect of Kat2a?}

In order to investigate possible gain-of-function effects, we designed an adeno-associated viral vector for overexpression of Kat2a, harboring a C-Terminal MYC-tag (AAV-Kat2a-MYC).

Using Kat2a specific primers for qRT-PCR as well as a MYC-specific antibody for IHC, we confirmed robust, DG-confined overexpression on the mRNA and protein level, respectively, which was about 5-fold overexpressed 14 days after injection compared to hippocampi either injected with AAV-GFP or AAV-Hdac2-MYC. This time course paralleled other AAV-mediated overexpression experiments in the host laboratory (Figure 3-11) (Bahari-Javan et al., 2012; Kerimoglu et al., 2013). Comparison of mRNA expression levels of both Kat2a and Hdac2 during overexpression by two different constructs yielded proof for expression while showing that overexpression of Kat2a did not result in compensatory upregulation of Hdac2 and VICE VERSA (Figure 3-11and Figure 6-2).

Fourteen days after injection, we subjected mice to a behavioral test battery to test for functional consequences of this overexpression. Basal anxiety, exploratory behavior and working memory of these mice were normal and comparable between groups. In addition, mice showed comparable amounts of freezing in response to contextual fear conditioning. The NOR task also did not reveal any differences. Mice of both groups failed to show preference for the novel object during longterm memory testing. This may indicate that a general negative effect of either surgery or viral injection or both on this type of memory testing. Yet, if we assume this memory type to be hippocampus-independent as discussed earlier (see 4.1.1), AAV injection into the DG cannot be the reason. It is possible, however, that small areas of cortical tissue are damaged during surgery and that these parts are necessary for long-term object recognition memory.

In the MWM we could detect a spatial-memory-impairment phenotype. Mice overexpressing Kat2a performed significantly worse in this test compared to the control group. However, there are several caveats for the interpretation of these results. First of all, the group size for behavioral experiments was rather small. This increases the chances of high variability in the sample group. Indeed, statistical testing for preference for the target quadrant in the control group did not reach the threshold of $p<0.05$ due to high variability. Second, the virus was injected into the DG and not the CA1, where LTP and expression analysis were carried out on. The decision about the intrahippocampal injection site was made due to the relatively lower expression in CA1 compared to DG. It is possible that differential expression in the different hippocampal subregions is necessary for normal function of the structure as a whole. Furthermore, this result could indicate a dominant negative effect of the overexpressed construct by replacing endogenous Kat2a from the SAGA and ATAC complexes while at the same time being non-functional due to the MYC-tag. The tag might also interfere with the Kat2a bromodomain that lies close to the C-terminus of the protein (Figure 1-2). However, this seems rather unlikely because the MYC-tag is only 10 AA long and the other tasks, including hippocampus-dependent contextual fear conditioning, did not yield any evidence for impaired function. A last but equally important caveat is the observation that AAV-Kat2ainjected mice did not actually perform poorly in the MWM. Remarkably, the control AAV-GFPinjected mice reached an average escape latency that was already below $10 \mathrm{~s}$ on day 7 , rendering a 
probe test for day 8 feasible. Also the AAV-Kat2a-injected mice showed a remarkable decrease in escape latency, yet not as fast as controls. Thus, this testing batch might resemble a group with an extraordinarily high performance with the AAV-GFP-injected mice at the top. Therefore, to get a conclusive answer, whether Kat2a overexpression in the DG is detrimental, the experiments needed to be conducted with a larger sample size.

In summary, though some caveats have to be taken into consideration, our results from the Kat2a gain-of-function approach demonstrate a functional role of Kat2s spatial memory, supporting the findings from the loss-of-function approach. Since both, gain- and loss-of-function yield similar results with respect to spatial memory, Kat2a function might be dose-dependent. However, there is no evidence in the literature to support this impression and additional experiments should be conducted with CA1-specific overexpression and further investigation of functionality of the construct in terms of functional integration into SAGA and ATAC complexes, binding to genomic regions and histone acetylation. 


\subsection{Extensive Transcriptional Changes and Decreased HDAC Activity in the Aging Hippocampus}

\subsubsection{Decreased HDAC activity towards the end of lifespan}

While HAT activity was similar in naïve young and aged mice, we found a negative correlation between age and downregulation of HDAC activity in mice at the end of their life span ( $\geq 28$ months, EOL) (Figure 3-14A,B). This came as a surprise for two reasons. First, decreased HDAC activity is generally associated with improvement of cognitive abilities and neuroprotection, and increased HDAC activity with age-associated, neurodegenerative diseases (Gräff and Tsai, 2013b; Kosik et al., 2012; Stilling and Fischer, 2011), which is clearly not the case in EOL mice. Though we did not test memory in these mice, it is highly unlikely that cognitive abilities improve again with further increasing age. Second, we could not detect global functional consequences of the decreased activity with regard to histone acetylation: the three investigated sites (H4K12ac, H3K9ac, H3K14ac) did not show alterations in bulk acetylation in hippocampi of EOL mice (Figure 3-14D). Also, expression of two key nuclear HDACs, namely Hdac2 and Hdac3, remained unchanged on the protein level (Figure 3-14C).

However, there are multiple explanations for this observation. First, decreased activity is measured at the enzymatic level, which indicates endogenous inhibitory regulation in the lysates. Indeed, endogenous inhibitors of HDAC activity haven been identified, including butyrate and $\beta$ hydroxybutyrate (Candido et al., 1978; Shimazu et al., 2013), pyruvate (Thangaraju et al., 2006), sphingosine-1-phosphate (S1P, mediated by SphK2 activity) (Hait et al., 2009; Spiegel et al., 2012) and mechanisms mediated by reactive oxygen species (ROS) (Druz et al., 2012; Rahman et al., 2004). In addition, HDAC as well as SIRT activity are coupled to the metabolic state of the cell via the metabolites Ac-CoA and NAD+, respectively (Ladurner, 2006). One or more of these mechanisms may be involved. Oxidative-stress-related mechanisms are particularly likely to affect HDAC activity in aged neurons and glia, since ROS are produced in larger amounts in old than in young cells (Muller et al., 2007).

An alternative interpretation is a compensatory mechanism at work in hippocampal cells. As the genome and chromatin stability is deteriorating with increasing age (see discussion in 4.2.3), it might become favorable for a cell to downregulate HDAC activity to maintain genomic plasticity.

Our results are in striking contrast to published data. A recent study by Zeng ET AL. suggested decreased BDNF expression in the rat hippocampus in association with decreased H3K9 and H4K12 acetylation (Zeng et al., 2011). Several other studies have also found decreasing levels of histone acetylation with aging. We found neither any evidence for reduced BDNF expression nor for decreased histone acetylation in the aging murine hippocampus. If at all, there were slight, nonsignificant increases in bulk histone acetylation at the sites we looked at (H3K9, H3K14 and H4K12); possibly connected to decreased HDAC activity. However, one has to consider the different experimental approaches, i.e. targeted, localized studies vs. genome-wide and bulk level experiments. 
A possible explanation for the discrepancies may arise from the fact that the assay used cannot discriminate between HDAC and SIRT activity. In fact, increasing activity of the SIRTs has in been shown to protect from age-associated damage and promote life span extension (Kanfi et al., 2012; Michan and Sinclair, 2007; Oberdoerffer et al., 2008), which is in line with our observation of decreasing activity towards the end of murine life span. Using SIRT and HDAC selective activity assays, it will be exciting to see whether decreased SIRT activity is the main component of the observed effect. This would also explain our finding of non-changing histone acetylation, since the nuclear SIRTs mainly deacetylate H4K16, which was not tested here.

\subsubsection{Memory enhancing effects of HDACi}

Genetic or pharmacological HDAC inhibition (HDACi) has been proven to ameliorate memoryassociated phenotypes in several animal models for neurological conditions, such as Alzheimer's disease, ALS, Huntington's disease, and stroke (Fischer et al., 2007; Francis et al., 2009; Govindarajan et al., 2011, 2012; Kilgore et al., 2009; Peleg et al., 2010; Ricobaraza et al., 2009) and could even enhance memory skills in young, healthy wild type mice (Fontán-Lozano et al., 2008; Guan et al., 2009). Though the latter might be an artifact of conventional mouse housing due to deprivation of sensory stimuli, the host laboratory could recently show, that the HDAC inhibitor SAHA could restore spatial and aversive memory in aged (16 months old), healthy wild type mice, when infused intrahippocampally (Peleg et al., 2010). Based on these findings, we hypothesized that pharmacological HDACi may also improve aging-associated memory impairment (AAMI) in a mouse model for advanced aging.

We administered SAHA orally, dissolved in DMSO and mixed with peanut butter. This approach is a lot less stressful, especially for mice at the advanced age of $23-24$ months. We could observe a rapid (ca. $10 \mathrm{~min}$ ) and complete ingestion of the $300 \mathrm{mg}$ peanut butter-SAHA dose offered each day for 3 weeks, which is a time frame chosen based on successful i.p. experiments in younger mice (Guan et al., 2009). Interestingly, this additional high-calorie diet did not lead to an overall increase in body weight. While, there was a significant effect of time in the daily body weight measurements, this effect was attributed to individual days only and is likely a result of daily variation.

To assess possible effects of SAHA on memory, we chose to perform the NOR test because it offers several advantages over other tests of the battery, like intragroup testing of memory and the absence of negative extrinsic motivation factors (e.g. aversion towards a context in FC). However, we modified the protocol slightly to meet our observation that aged mice show a lack of motivation to explore the objects when habituated for too long. Since we administered SAHA systemically, it was irrelevant whether our NOR paradigm was truly hippocampus-dependent. Our results clearly demonstrate memory impairment in the vehicle group in both, STM and LTM paradigms, thereby confirming age-associated memory impairment. While STM was unchanged in SAHA-treated animals, we could observe a moderate improvement in LTM, indicating a transcription-dependent mechanism of action (Figure 3-16B). However, this finding raises the question of, how mice with impaired STM can form an LTM trace. The answer may very well lie in the way the experiment was conducted, since the STM test can also be viewed as an additional training session for the LTM test. 
In a control experiment we could prove that SAHA crosses the blood brain barrier and has a transient positive effect on hippocampal H4K12ac for at least 1 hour after i.p. injection (Figure 3-15). In fact, we expected histone acetylation to be increased for a longer period, since the drug was applied chronically over 1 week. If we assume a similar time course after oral administration, daily SAHA will cause repeated waves of increased histone acetylation rather than a chronically high level of acetylation. It can be speculated that these waves may result in differential transcriptional profiles in the brain, if repeated over a certain time (e.g. 3 weeks).

To my knowledge, our results for the first time demonstrate functional oral application of SAHA in the context of mammalian learning and memory and thereby underline the therapeutic potential of HDACi in AAMI.

\subsubsection{Transcriptional Regulation in the Hippocampus: Immune System and Chromatin}

Though brain aging also goes along with massive transcriptional changes, a detailed, homogenous picture of the transcriptome of the aging mouse hippocampus, especially towards the end of an individual's life span, has not been drawn yet.

Using two different techniques we could determine the transcription profile of the aging hippocampus. Both techniques showed a robust overlap within age-upregulated genes, suggesting a good fit between methods and most variability can be attributed to age rather than to technical issues. Furthermore, overlapping enrichment of functional categories suggests the induction of a distinguished transcriptional program that is characteristic of aging. This was also confirmed by analyzing functional enrichment in a merged list of upregulated genes during aging independent of the specific age group ( $\geq 24$ months). Though genes sets regulated in the different aging groups were not exactly the same, similar pathways were regulated. This is a general theme in transcriptional regulation and it also points to the fact that the changes are not due to random deterioration of transcription, but are part of a regulated process. If so, what is the starting point of this process? Does this also imply that aging is an evolutionary programmed process rather than the effect of incremental damage over time? These and other questions have been asked repeatedly in the field of aging research and to answer them is far beyond the scope of this thesis. What this study, however, can provide is important insight into the specific transcriptional changes that are associated with the aging process. If these changes are the cause or links in a long chain of consequences, remains a matter of ongoing research.

Based on the gene expression profile, the induced program is highly associated with the immune system. Several functional categories were highly enriched among age-upregulated genes, the most significant one being "positive regulation of immune response" (Table 3-6). Interestingly, a number of these genes are also implicated in autoimmune diseases like systemic lupus erythematosus, i.e. they may play a role in self-directed, destructive inflammatory processes also in the aging brain. These genes belong to the complement cascade, which is an important subsystem 
of the innate immune system. The specific implications of this particular group of genes in the aging process will be discussed below (see 4.2.4)

Interestingly, and in parallel to results from the Kat2a-loss-of-function expression study, the overlap among downregulated genes was considerably less pronounced. This is likely due to the low number of replicates $(n=3)$ in the comparison of $3 m$ vs. $28 m$ using RNA-seq, but it may also reflect that the pattern of downregulation is not as strictly programmed as that of upregulation. As a matter of fact, interindividual as well as cell-to-cell variation is commonly observed with increasing age of an organism (Bahar et al., 2006; Li et al., 2009b; Somel et al., 2006; Southworth et al., 2009; Welle et al., 2003). However, our data does not yield any evidence for higher variability in samples prepared from aged tissue (PCA, sample distance plots, Figure 3-17)

However, we were able to discover some striking gene sets to be downregulated. These included chromatin-related genes such as histone-coding genes. It has recently been described that aging is associated with reduced histone biosynthesis and elevated histone expression promotes life span extension (Feser et al., 2010; O'Sullivan and Karlseder, 2012; O'Sullivan et al., 2010), which supports our observation. Apart from downregulated histone genes, a highly upregulated chromatin-related gene codes for the long non-coding RNA (IncRNA) Neat1. This gene was found in all samples from aging mice, independent of the method used. Neat 1 is an interesting gene, since it is necessary for nuclear paraspeckle formation (Bond and Fox, 2009; Naganuma and Hirose, 2013). It may therefore be implicated in changes in nuclear architecture that are evident during aging (Barascu et al., 2012; Taimen et al., 2009). Along with Neat1, three other genes involved in paraspeckle formation were differentially regulated ( $P s p c 1$, downregulated, CPSF6, upregulated, Malat1, another IncRNA, upregulated), further supporting the importance of this recently discovered structure in hippocampal aging. Interestingly, increased paraspeckle formation was also linked to viral infection of the central nervous system (Bond and Fox, 2009; Saha et al., 2006), providing an additional link to immune-response-related mechanisms to be upregulated with aging. Paraspeckles may also be involved in cotranscriptional mRNA processing and splicing (Bond and Fox, 2009; Fox et al., 2002). As the brain is a major site of alternative splicing (Dillman et al., 2013; Li et al., 2007), it does not come as a surprise that also brain aging may be associated with differential splicing, as several recent studies suggest (Harries et al., 2011; Mazin et al., 2013; Meshorer and Soreq, 2002; Muratore et al., 2013; Osorio et al., 2011; Tollervey et al., 2011). Further analyses of the data with respect to differential splicing events are necessary to determine whether specifc program becomes evident or whether it provides insights into the reasons for the observed transcriptional changes.

In general, the results are in line with other studies on transcriptional changes in the hippocampus during aging (e.g. Blalock et al., 2010 (primate) or Verbitsky et al., 2004 (mouse)), since they have several genes and functional category enrichment in common (e.g. immune response). Yet, some features are constrain commonalities. For example, Blalock et al. found chromatin-related gene to be upregulated, while we and others find several chromatin constituents to be downregulated with aging (Blalock et al., 2010; O'Sullivan and Karlseder, 2012). 


\subsubsection{Aging, Alzheimer's disease and the complement connection}

For two genes, $C 4 b$ and $G F a p$, we could confirm upregulation using QRT-PCR and on the protein level. qRT-PCR results resembled fold changes from genome-wide methods very closely, thereby validating these. Furthermore, the genome-wide methods validate each other in our aging study.

Gfap upregulation is clearly an effect of astrocyte invasion, as seen by immunostaining. Astrocytic hypertrophy in the brain is an established effect during aging and has been described extensively (reviewd by Finch and Morgan, 1990). Based on similar findings, Blalock and collegues have speculated that increased numbers of glia may be eiter a cause or a consequence of immune gene induction (Blalock et al., 2003).

In this respect, $\mathbf{C} \boldsymbol{4} \boldsymbol{b}$ is an interesting gene from many aspects. First, it was already found to be upregulated as one of the very few DEGs in the comparison of hippocampal expression in 3-monthold mice with 16-month-old mice (3-fold upregulation) (Peleg et al., 2010). Interestingly, out of the 12 upregulated genes found in this independent study, 6 genes were also found upregulated in the present study. In addition, other studies have observed complement-gene upregulation in early studies of aging in the hippocampus as well as in the prefrontal cortex (Bordner et al., 2011; Reichwald et al., 2009; Verbitsky et al., 2004). Along with $C 4 b$ and $C 3$ - another complement component - these were 1700112E06Rik, BC061194, Cox8b and Sult1c2. While the latter two of these four genes have a described function - notably, Cox8b is a cytochrome involved in oxidative phosphorylation and associated with neurodegenerative diseases - the former two transcripts have no known function. However, these 6 genes obviously belong to a set of genes that are highly associated with murine hippocampal aging.

Human aging is the strongest risk factor for Alzheimer's disease (AD) (Lopez, 2011). The reason why late-onset $A D$ (LOAD) is associated with increasing age is not yet fully understood. However, accumulating evidence links LOAD to an increasing number of genetic risk factors and it seems feasible that these genetic risk factors result in LOAD due to changes at the transcriptome and proteome level. Indeed, in our hippocampal aging study in mice, we found upregulation of several homologs, interaction partners or other closely related genes to almost all genes that appear to be the top 10 single genetic risk factors for LOAD as designated by the AlzGene database ${ }^{26}$ (Table 4-1) (Bertram et al., 2007).

\footnotetext{
${ }^{26}$ http://www.alzgene.org/TopResults.asp, Accessed: 25.03.2013
} 
Table 4-1: Comparison of results from genetic association studies for LOAD from the AlzGene database with differential gene expression in the aging mouse hippocampus. All "Related genes" were upregulated with aging.

\begin{tabular}{|c|c|c|}
\hline $\begin{array}{c}\text { AlzGene Top } 10 \\
\text { Results }\end{array}$ & $\begin{array}{c}\text { Related genes } \\
\text { (homologs, direct interaction partners or } \\
\text { involved in similar function) }\end{array}$ & Group/ Function \\
\hline$A P O E_{-} e 2 / 3 / 4$ & $\begin{array}{c}\text { Apobec1 } \\
\text { Apobr } \\
\text { Apod } \\
\text { Apol9a } \\
\text { Apol9b }\end{array}$ & $\begin{array}{l}\text { Apolipoproteins, } \\
\text { receptors or } \\
\text { transcript } \\
\text { modifying }\end{array}$ \\
\hline BIN1 & - & $\begin{array}{l}\text { transcriptional } \\
\text { regulation }\end{array}$ \\
\hline$C L U$ & see $A p o E$ & $\begin{array}{l}\text { Apolipoproteins } \\
(\text { Clu = ApoJ }) \\
\text { regulator of } \\
\text { complement } \\
\text { cascade }\end{array}$ \\
\hline$A B C A 7$ & see $A p o E$ & $\begin{array}{l}\text { binds to } \\
\text { Apolipoproteins }\end{array}$ \\
\hline \multirow[t]{9}{*}{ CR1 } & C1qa & \\
\hline & $c 1 q b$ & \\
\hline & C1qc & \\
\hline & C1ra & Complement \\
\hline & C2 & system, innate \\
\hline & C3 & immune system \\
\hline & C3ar1 & \\
\hline & $c 4 a$ & \\
\hline & $c 4 b$ & \\
\hline PICALM & - & $\begin{array}{c}\text { clathrin-mediated } \\
\text { endocytosis }\end{array}$ \\
\hline$M S 4 A 6 A$ & $M s 4 a 6 b$ & $\begin{array}{l}\text { Transmembrane } \\
\text { proteins, immune } \\
\text { system }\end{array}$ \\
\hline$C D 33$ & $\begin{array}{l}\text { Cd33 } \\
\text { cd22 }\end{array}$ & $\begin{array}{c}\text { lectins, immune } \\
\text { system }\end{array}$ \\
\hline \multirow[t]{2}{*}{ MS4A4E } & $M s 4 a 4 b$ & Transmembrane \\
\hline & $M s 4 a 4 c$ & $\begin{array}{c}\text { proteins, immune } \\
\text { system }\end{array}$ \\
\hline$C D 2 A P$ & $\mathrm{Cd} 2$ & immune system \\
\hline
\end{tabular}

Hence, the second reason to look at $C 4 b$ in more detail is its role as a complement component. The complement system is part of the innate immune system and is used for lysis of pathogenic and other cells, activation of inflammatory responses, as well as for clearance of antibody complexes and other extracellular aggregates (Czirr and Wyss-Coray, 2012). Activation and regulation of the complement cascade, also in the brain, are well studied but complex and often controvertial (for a review see Czirr and Wyss-Coray, 2012). Supplementary Figure 6-4 shows the KEGG pathway of the complement and coagulation cascades and the differentially expressed genes found in this study. It is important to understand that C4 as well as the C3 proteins are critical relay nodes that are extracellularly processed upon activation. The products of this process will in turn lead to further downstream activation of the cascade and ultimately to the assembly of the membrane attack complex. A key negative regulator of the cascade is the complement receptor $\mathbf{1}$ (CR1), encoded in 
the mouse by the $C r 2$ gene ${ }^{27}$ (Jacobson and Weis, 2008). CR1 binds processed C4 and C3 and leads to degradation of the products by serving as a cofactor for their cleavage or receptor internalization (Krych-Goldberg and Atkinson, 2001). Interestingly, in human populations there is a high degree of variability and several alleles are present. Both sources of this variability, single nucleotide polymorphisms (SNPs) and copy number variations (CNVs) in the human CR1 gene have been shown to be highly associated with LOAD in a number of studies (Bertram et al., 2007; Biffi et al., 2010, 2012; Brouwers et al., 2012; Carrasquillo et al., 2010; Chibnik et al., 2011; Crehan et al., 2012; Hazrati et al., 2012; Keenan et al., 2012; Lambert et al., 2009). This makes it very tempting to draw the conclusion that increased expression of C3 and C4 with aging will result in aberrant regulation of the complement cascade and in turn to neurodegeneration in carriers of the "wrong" CR1 allele. Aberrant regulation could lead to neurodegeneration in two different, non-mutual exclusive ways. On the one hand, differential CR1 regulatory activity could lead to less effective Aß aggregate clearance (Fonseca et al., 2004). On the other hand, deregulated activation of the complement system could result in collateral damage by overactive inflammation (reviewed in (Czirr and WyssCoray, 2012).

The exact mechanism of altered CR1 regulatory function in this complex network is still to be elucidated and a beneficial effect of complement inhibition has been demonstrated (Fonseca et al., 2004; Kulkarni et al., 2008, 2011; Leinhase et al., 2006; Pillay et al., 2008; Rancan et al., 2003) but remains controversial (Loeffler, 2004; Maier et al., 2008; Wyss-Coray et al., 2002). Thus, targeting the complement system and other inflammatory pathways poses an intriguing possibility for the treatment of aging-associated diseases and cognitive decline. Physical activity in rodents and humans has been shown to improve cognitive abilities during aging (Erickson et al., 2011; van Praag et al., 1999; Praag et al., 2005). Remarkably, a recent study could show in aged mice that voluntary wheel running leads to a reduction in $C 4 b$ expression in the hippocampus (Kohman et al., 2011), demonstrating a clear correlation between C4 levels and cognitive abilities, which is further supported by the finding that $\mathrm{C} 4$ inhibition by vaccinia virus complement control protein has beneficial effects on memory performance in mouse models of Alzheimer's disease (Kulkarni et al., 2008, 2011; Pillay et al., 2008).

Interestingly, we found evidence for the involvement of H3K9ac in the upregulation of C4b using ChIP-seq data for this modification. This nicely demonstrates the power of such a genome-wide approach, since intronic enrichment of $\mathrm{H} 3 \mathrm{~K} 9 \mathrm{ac}$ would have remained unexplored, if we had performed regular ChIP experiments with subsequent qRT-PCR, targeting the $C 4 b$ promoter. However, further validation of this finding by QRT-PCR and correlation with other histone modifications or DNA methylation will be necessary to establish a clear involvement of chromatindependent upregulation of complement-associated genes.

\footnotetext{
${ }^{27}$ The $C r 2$ gene in non-primate mammals produces two isoforms, CR1 and CR2, by alternative splicing. The primate CR1 receptor likely evolved form duplications of another gene, Cr1la.k.a. Crry. Both gene products can bind and regulate processed C4 and C3 (Jacobson and Weis, 2008).
} 
In conclusion, our data strongly suggest an extensive upregulation of immune-system-related genes with aging, that may, at least in part be regulated by altered chromatin-dependent mechanisms, including reduced synthesis of new histones and other chromatin components on one hand, and upregulation of histone acetylation at the $c 4 b$ locus on the other. Reduction of inflammatory damage may in turn result in improvement of cognitive abilities in aged mice and humans. Upregulation of the complement cascade with age has been observed repeatedly before, but its significance for non-pathological aging may have been overlooked or neglected. Now, in the light of recent genome-wide association studies for LOAD risk loci, this fact deserves new attention. 


\subsection{Closing remarks and Outlook}

\subsubsection{Technical considerations}

Any immunological experiments to determine the abundance of localization of a certain histone modification depend on antibody specificity. To date most histone-modification antibodies are not fully determined in their specificity. Commercial vendors offer validation using multiple measures of specific binding to a certain histone modification. However, these tests often neglect the effect of combinatorial modifications that are very likely to occur on any histone. For example, Histone $\mathrm{H} 4$ may be acetylated on $\mathrm{K} 12$ and at the same time at $\mathrm{K} 5$. It cannot be easily inferred whether an antibody that is "specific" to H4K12ac would recognize this histone or whether it only recognizes $\mathrm{H} 4$ that is acetylated at K12 exclusively. This strongly depends on the type and length of the immunogen that is used. Mostly the immunogen is a short peptide carrying a modified lysine residue surrounded by several amino acids in the sequence of a conserved histone tail. This effect always has to be taken into consideration when interpreting western blot, IHC or ChIP-seq results.

Short-read sequencing results in millions of short DNA sequences that need to be aligned to a reference sequence. For our data we used Bowtie2 that aligns reads to the mouse genome. However, alignment cannot account for splice junctions, since the genomic reference sequence also contains all introns that are not present in the sequenced cDNA reads. We used a very sensitive local alignment mode of Bowtie2 to circumvent this problem to the largest possible extent and retrieved very good alignment rates. However, recent developments in bioinformatics short-read aligning for RNA-seq data may produce even better alignments and will also provide more detailed information about splicing events, that were not analyzed in this thesis (Trapnell et al., 2009). As with all largescale experiments, a further caveat in using NGS-based approaches for differential expression analysis is the generation of artifacts like false positive DEG calling. A likely artifact is calling of small RNAs as DEGs, since these often lie within the gene bodies of other genes They should, however, be excluded by poly-dT pulldown and also by size selection step in library preparation. This structural error in RNA-seq becomes more critical in complex mammalian genomes.

A limiting factor in the search for enriched functional categories is the amount of data stored in databases that are queried for the analysis. Only genes with annotated function can be found in these databases and can contribute to representation. Furthermore, genes are often annotated in the context of a particular treatment or cell type, tissue or disease. The more GO-Terms or pathways are associated with a certain gene, the better studied this gene is. This correlation could be the result of a sample bias but it is also possible that this gene is an important node in one or more regulatory networks and is well studied because it is readily found in multiple experimental settings. 


\subsubsection{The role of histone acetylation in learning and memory}

As discussed above, the results of our experiments on the role of Kat2a in learning and memory and stimulus-dependent gene expression open further questions. To address these questions additional analyses would need to be done, including a more focal knockout of Kat2a using AAV-Cre mediated recombination, which would exclude all developmental and non-hippocampus dependent effects of the knockout (McQuown and Wood, 2011). Furthermore, recently evidence is accumulating that not only transcriptional initiation but also elongation and even stimulus-dependent co-transcriptional alternative splicing is heavily influenced by chromatin status and histone modifications, including histone acetylation (Ai et al., 2011; Ginsburg et al., 2009; Johnsson and Wright, 2010; Kim et al., 2002; Schor et al., 2009; Tyagi et al., 2009; Zhou et al., 2011b). Thus, it would be intriguing to analyze our RNA-seq data set on the level of individual exons to assess differential transcript abundance in response to novelty exposure.

Though more and more evidence is gathered to determine a role for histone acetylation in learning and memory most of the data so far is correlative. In particular, concerning the molecular function of certain histone sites there is no definite proof for functional relevance of those modifications in multicellular organisms. To this end, functional studies in yeast (Dai et al., 2008; Dion et al., 2005; Durrin et al., 1991), where specific mutant histone genes are introduced to study the functional consequences of these mutations, need to be advanced to higher organisms. A promising model organism for these kinds of studies, especially in the context of learning and memory is DROSOPHILA MELANOGASTER. Here, genetic tools have been developed and started being used for applied functional studies (Gunesdogan et al., 2010; Pengelly et al., 2013). It is exciting to think of making use of these tools in the context of learning and memory.

\subsubsection{Implications for the synaptic tagging and capture hypothesis}

As shown in this and many studies HDACi as well as HAT activation have a positive effect on longterm memory consolidation. The reason for this is likely to be found in the stimulation of expression of genes necessary for long-lasting changes in synaptic plasticity. Some studies have shown that HDACi is able to transform early-LTP into transcription-dependent forms of late-LTP (Vecsey et al., 2007; Wood et al., 2005), which led us to propose that dynamic histone acetylation may be involved in synaptic tagging and capturing (STC) (Stilling and Fischer, 2011). This thesis provides further support for the proposed implication of HDACi and HAT activity in transcription-dependent capturing of synaptic tags. This view also helps to understand the specific effect of SAHA treatment in long-term object recognition. Here, the STM test could result in setting the synaptic tag, while SAHA is involved in transcription-dependent capturing. In addition, our results regarding impaired LTP induction in Kat2a cKO mice support the STC hypothesis. Figure 4-1 summarizes a possible extension of the STC hypothesis and its implications for HDACi and HAT activity to facilitate memory consolidation. While Wood and colleagues have come to similar conclusions and have formulated the "molecular brake pad hypothesis of HDAC function" as an explanation for these intriguing observations (McQuown and Wood, 2011; Vogel-Ciernia and Wood, 2012), Fontán-Lozano 
et al. take the same line in hypothesizing "[...] that systemic administration of HDAC inhibitors decreases the stimulation threshold necessary for cognitive processes [...]" (Fontán-Lozano et al., 2008). In this way, HDAC inhibitors on a molecular parallel the electrophysiological effects of transcranial direct current stimulation (Nitsche et al., 2003).

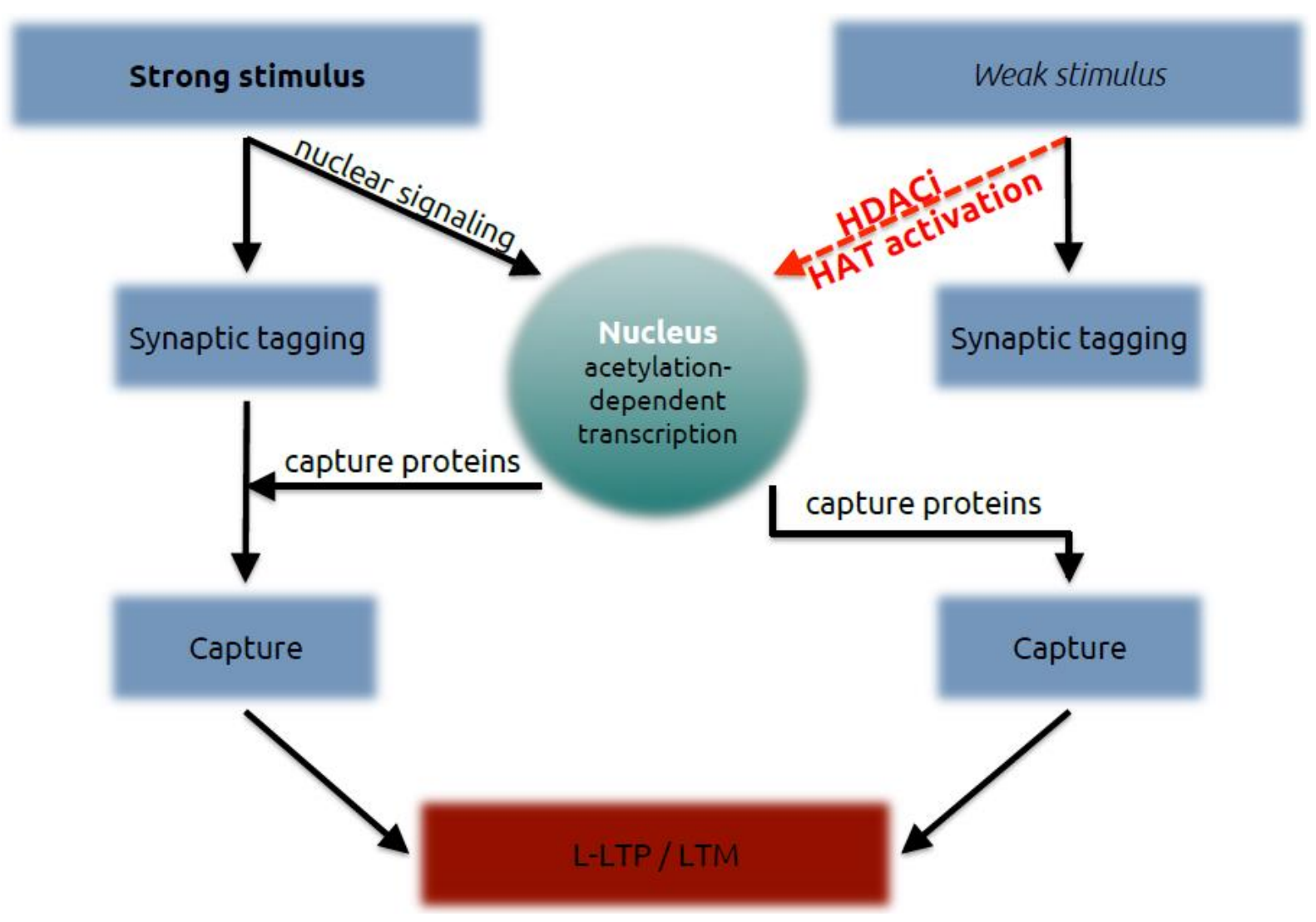

Figure 4-1: The role of histone acetylation in synaptic tagging and capturing. HDACi or HAT activation bridge nuclear signaling after a weak stimulus and regulate the production of proteins needed for capturing. Thereby a weak stimulus can result in late LTP (L-LTP) and LTM consolidation.

\subsection{4. (Brain) Aging - an Aggregopathy? An Autoimmune Disease? Or Both!?}

During the last century, multiple theories of aging have been developed, with different degrees of validity, advantages and shortcomings. These theories are in part also based on findings resulting from new genome-wide transcriptional profiling techniques. In spite of general agreements (with the mentioned exceptions) of our data with previous studies, these studies have lead to different, sometimes opposing, interpretations. While it would go far beyond this thesis to discuss all theories here in detail, I want to contribute to this ongoing discussion by drawing attention to one of these theories, since it may help to understand certain aspects of my interpretation of the data described earlier.

Pleiotropy describes a mechanism by which a gene can have more than one function. Alternatively a particular gene function can have diverse effects, depending on the situation (Stearns, 2010). A special case of pleiotropy is antagonistic pleiotropy, where these two functions or outcomes 
phenotypically antagonize each other. It has been proposed that antagonistic phenotypes may present with a substantial time lag within an organism's life span. For example, a certain gene has a beneficial or even necessary role during development or early life but becomes detrimental during later phases in life. Natural selection will most likely only be able to act on the early-life effects of this gene, so that detrimental effects may accumulate in the population. The tumor suppressor p53 has been suggested to be a gene with such antagonistic functions in youth and aging (Rodier et al., 2007).

Results from this study suggest that many immune-related genes may have antagonistic pleiotropic effects, especially in the context of brain aging. While a functional immune system confers defense against various parasites and defective or degenerated cells and presents an evolutionary advantage, deregulated immune cascades lead to autoimmunity and collateral damage in inflammated tissue. This hypothesis could be tested by applying anti-inflammatory agents starting from a certain age (Jang et al., 2010; Leinhase et al., 2006). Interestingly, Blalock and colleagues favor a similar model for the implications of inflammation in brain aging: Demyelination may lead to higher myelin turnover, which results in autoimmune inflammatory cascades against new myelin antigens involving astrocytes and microglia (Blalock et al., 2003). From this point of view, myelincomposing proteins exhibit antagonistic pleiotropy.

A related concept accounts for the fact that many age-related diseases are associated with the accumulation of insoluble intra- or extracellular aggregates (e.g. Aß-Plaques, Neurofibrillary Tangles, Lewi Bodies, SOD-accumulates, Huntingtin-aggregates). Also refered to as proteopathies (Walker and LeVine, 2000), the term aggregopathy (Dohm et al., 2008) tries to group these diseases based on this commonality. During aging oxidative damage of macromolecules also leads to accumulation of aggregates (e.g. Lipofuscin) that may result in impaired cellular functionality and eventually degeneration. Consequently aging could be termed an aggregopathy.

The two concepts, antagonistic pleiotropy and aggregopathies, may in fact be interconnected. Immune-system function may be directed at clearing aggregates and abnormally functioning cells but would destroy surrounding tissue during this process (see (Czirr and Wyss-Coray, 2012). The role of the complement system in clearance of antibody complexes would be especially important from this point of view. Testing this hypothesis however is not straightforward since inhibiting immune function could also result in increased accumulation of aggregates which would be detrimental in itself.

Aging has many facets and age-associated memory impairment is most likely caused by several of these factors. However, it remains one of the fundamental challenges of aging research to determine causes and consequences of the aging process. This study is supposed to offer some insights that may deserve a closer look and hints towards directions for further exploration. I personally would be more than pleased to see future research revealing that this thesis has contributed to elucidating a tiny part of one of the greatest puzzles of all: How and why do we age? 


\section{References}

Agis-Balboa, R.C., Arcos-Diaz, D., Wittnam, J., Govindarajan, N., Blom, K., Burkhardt, S., Haladyniak, U., Agbemenyah, H.Y., Zovoilis, A., Salinas-Riester, G., et al. (2011). A hippocampal insulin-growth factor 2 pathway regulates the extinction of fear memories. EMBO J 30, 4071-4083.

Ai, N., Hu, X., Ding, F., Yu, B., Wang, H., Lu, X., Zhang, K., Li, Y., Han, A., Lin, W., et al. (2011). Signal-induced Brd4 release from chromatin is essential for its role transition from chromatin targeting to transcriptional regulation. Nucleic Acids Res.

Allfrey, V.G., Faulkner, R., and Mirsky, A.E. (1964). ACETYLATION AND METHYLATION OF HISTONES AND THEIR POSSIBLE ROLE IN THE REGULATION OF RNA SYNTHESIS*. Proc Natl Acad Sci U S A 51, 786-794.

Allis, C.D., Berger, S.L., Cote, J., Dent, S., Jenuwien, T., Kouzarides, T., Pillus, L., Reinberg, D., Shi, Y., Shiekhattar, R., et al. (2007). New Nomenclature for Chromatin-Modifying Enzymes. Cell 131, 633-636.

Anagnostaras, S.G., Gale, G.D., and Fanselow, M.S. (2001). Hippocampus and contextual fear conditioning: recent controversies and advances. Hippocampus 11, 8-17.

Anamika, K., Krebs, A.R., Thompson, J., Poch, O., Devys, D., and Tora, L. (2010). Lessons from genome-wide studies: an integrated definition of the co-activator function of histone acetyl transferases. Epigenetics Chromatin 3, 18.

Anders, S., and Huber, W. (2010). Differential expression analysis for sequence count data. Genome Biol. 11, R106.

Andersen, P., Morris, R., Amaral, D., and O’Keefe, J. (2007). The hippocampus book (Oxford University Press US).

Andrade, R. (1998). Regulation of membrane excitability in the central nervous system by serotonin receptor subtypes. Ann. N. Y. Acad. Sci. 861, 190-203.

Arrowsmith, C.H., Bountra, C., Fish, P.V., Lee, K., and Schapira, M. (2012). Epigenetic protein families: a new frontier for drug discovery. Nat Rev Drug Discov 11, 384-400.

Arvanov, V.L., and Wang, R.Y. (1998). M100907, a selective 5-HT2A receptor antagonist and a potential antipsychotic drug, facilitates N-methyl-D-aspartate-receptor mediated neurotransmission in the rat medial prefrontal cortical neurons in vitro. Neuropsychopharmacology 18, 197-209.

Atkinson, R.C., and Shiffrin, R.M. (1968). Human Memory: A Proposed System and its Control Processes. In Psychology of Learning and Motivation, Kenneth W. Spence and Janet Taylor Spence, ed. (Academic Press), pp. 89-195.

Baddeley, A. (2003). Working memory: looking back and looking forward. Nat Rev Neurosci 4, 829-839.

Bahar, R., Hartmann, C.H., Rodriguez, K.A., Denny, A.D., Busuttil, R.A., Dollé, M.E.T., Calder, R.B., Chisholm, G.B., Pollock, B.H., Klein, C.A., et al. (2006). Increased cell-to-cell variation in gene expression in ageing mouse heart. Nature 441, 1011-1014.

Bahari-Javan, S., Maddalena, A., Kerimoglu, C., Wittnam, J., Held, T., Bähr, M., Burkhardt, S., Delalle, I., Kügler, S., Fischer, A., et al. (2012). HDAC1 Regulates Fear Extinction in Mice. J. Neurosci. 32, 5062-5073.

Ballarini, F., Moncada, D., Martinez, M.C., Alen, N., and Viola, H. (2009). Behavioral tagging is a general mechanism of long-term memory formation. Proc. Natl. Acad. Sci. U.S.A. 106, 14599-14604.

Bandeira, F., Lent, R., and Herculano-Houzel, S. (2009). Changing numbers of neuronal and non-neuronal cells underlie postnatal brain growth in the rat. PNAS 106, 14108-14113.

Barascu, A., Le Chalony, C., Pennarun, G., Genet, D., Zaarour, N., and Bertrand, P. (2012). Oxydative stress alters nuclear shape through lamins dysregulation: a route to senescence. Nucleus $3,411-417$.

Barco, A., Lopez de Armentia, M., and Alarcon, J.M. (2008). Synapse-specific stabilization of plasticity processes: the synaptic tagging and capture hypothesis revisited 10 years later. Neurosci Biobehav Rev 32, 831-851. 
Barker, G.R.I., and Warburton, E.C. (2011). When Is the Hippocampus Involved in Recognition Memory? J. Neurosci. 31, 10721-10731.

Benito, E., Valor, L.M., Jimenez-Minchan, M., Huber, W., and Barco, A. (2011). cAMP response element-binding protein is a primary hub of activity-driven neuronal gene expression. J. Neurosci. 31, 18237-18250.

Benjamini, Y., and Hochberg, Y. (1995). Controlling the false discovery rate: a practical and powerful approach to multiple testing. Journal of the Royal Statistical Society. Series B. Methodological 57, 289-300.

Berger, A., Venturelli, S., Kallnischkies, M., Böcker, A., Busch, C., Weiland, T., Noor, S., Leischner, C., Weiss, T.S., Lauer, U.M., et al. (2012). Kaempferol, a new nutrition-derived pan-inhibitor of human histone deacetylases. J. Nutr. Biochem.

Bert, B., Fink, H., Rothe, J., Walstab, J., and Bönisch, H. (2008). Learning and memory in 5-HT(1A)-receptor mutant mice. Behav. Brain Res. 195, 78-85.

Bertram, L., McQueen, M.B., Mullin, K., Blacker, D., and Tanzi, R.E. (2007). Systematic meta-analyses of Alzheimer disease genetic association studies: the AlzGene database. Nat. Genet. 39, 17-23.

Bhuiyan, M.M.R., Sato, M., Murao, K., Imachi, H., Namihira, H., Ishida, T., Takahara, J., and Miyauchi, A. (2001). Differential expression of menin in various adrenal tumors. Cancer 92, 1393-1401.

Biffi, A., Anderson, C.D., Desikan, R.S., Sabuncu, M., Cortellini, L., Schmansky, N., Salat, D., and Rosand, J. (2010). Genetic variation and neuroimaging measures in Alzheimer disease. Arch. Neurol. 67, 677-685.

Biffi, A., Shulman, J.M., Jagiella, J.M., Cortellini, L., Ayres, A.M., Schwab, K., Brown, D.L., Silliman, S.L., Selim, M., Worrall, B.B., et al. (2012). Genetic variation at CR1 increases risk of cerebral amyloid angiopathy. Neurology 78, 334-341.

Bishop, N.A., Lu, T., and Yankner, B.A. (2010). Neural mechanisms of ageing and cognitive decline. Nature 464, 529-535.

Blalock, E.M., Chen, K.-C., Sharrow, K., Herman, J.P., Porter, N.M., Foster, T.C., and Landfield, P.W. (2003). Gene Microarrays in Hippocampal Aging: Statistical Profiling Identifies Novel Processes Correlated with Cognitive Impairment. J. Neurosci. 23, 3807-3819.

Blalock, E.M., Grondin, R., Chen, K., Thibault, O., Thibault, V., Pandya, J.D., Dowling, A., Zhang, Z., Sullivan, P., Porter, N.M., et al. (2010). Aging-related gene expression in hippocampus proper compared with dentate gyrus is selectively associated with metabolic syndrome variables in rhesus monkeys. J. Neurosci 30, 6058-6071.

Blanchard, R.J., and Blanchard, D.C. (1969). Passive and active reactions to fear-eliciting stimuli. J Comp Physiol Psychol 68, 129-135.

Bliss, T.V., and Gardner-Medwin, A.R. (1973). Long-lasting potentiation of synaptic transmission in the dentate area of the unanaestetized rabbit following stimulation of the perforant path. J. Physiol. (Lond.) 232, 357-374.

Bliss, T.V.P., and Lømo, T. (1973). Long-lasting potentiation of synaptic transmission in the dentate area of the anaesthetized rabbit following stimulation of the perforant path. J Physiol 232, 331-356.

Bond, C.S., and Fox, A.H. (2009). Paraspeckles: nuclear bodies built on long noncoding RNA. J. Cell Biol. 186, 637-644.

Bordner, K.A., Kitchen, R.R., Carlyle, B., George, E.D., Mahajan, M.C., Mane, S.M., Taylor, J.R., and Simen, A.A. (2011). Parallel declines in cognition, motivation, and locomotion in aging mice: association with immune gene upregulation in the medial prefrontal cortex. Exp. Gerontol. 46, 643-659.

Bousiges, O., Vasconcelos, A.P. de, Neidl, R., Cosquer, B., Herbeaux, K., Panteleeva, I., Loeffler, J.-P., Cassel, J.C., and Boutillier, A.-L. (2010). Spatial Memory Consolidation is Associated with Induction of Several LysineAcetyltransferase (Histone Acetyltransferase) Expression Levels and H2B/H4 Acetylation-Dependent Transcriptional Events in the Rat Hippocampus. Neuropsychopharmacology.

Braidy, N., Guillemin, G.J., Mansour, H., Chan-Ling, T., Poljak, A., and Grant, R. (2011). Age related changes in NAD+ metabolism oxidative stress and Sirt1 activity in wistar rats. PLoS ONE 6, e19194.

Bramham, C.R., and Wells, D.G. (2007). Dendritic mRNA: transport, translation and function. Nat. Rev. Neurosci. 8, 776-789. 
Bromley-Brits, K., Deng, Y., and Song, W. (2011). Morris Water Maze Test for Learning and Memory Deficits in Alzheimer\&\#39;s Disease Model Mice. Journal of Visualized Experiments.

Brouwers, N., Van Cauwenberghe, C., Engelborghs, S., Lambert, J.-C., Bettens, K., Le Bastard, N., Pasquier, F., Montoya, A.G., Peeters, K., Mattheijssens, M., et al. (2012). Alzheimer risk associated with a copy number variation in the complement receptor 1 increasing C3b/C4b binding sites. Mol Psychiatry 17, 223-233.

Brownell, J.E., Zhou, J., Ranalli, T., Kobayashi, R., Edmondson, D.G., Roth, S.Y., and Allis, C.D. (1996). Tetrahymena Histone Acetyltransferase A: A Homolog to Yeast Gcn5p Linking Histone Acetylation to Gene Activation. Cell 84, 843-851.

Bruce, D. (2001). Fifty years since Lashley's In search of the Engram: refutations and conjectures. J Hist Neurosci 10, 308-318.

Bu, P., Evrard, Y.A., Lozano, G., and Dent, S.Y.R. (2007). Loss of Gcn5 Acetyltransferase Activity Leads to Neural Tube Closure Defects and Exencephaly in Mouse Embryos. Mol Cell Biol 27, 3405-3416.

Butte, A.J., Dzau, V.J., and Glueck, S.B. (2001). Further defining housekeeping, or "maintenance," genes Focus on "A compendium of gene expression in normal human tissues". Physiol. Genomics 7, 95-96.

Buzsáki, G. (1996). The hippocampo-neocortical dialogue. Cereb. Cortex 6, 81-92.

Cajigas, I.J., Tushev, G., Will, T.J., Tom Dieck, S., Fuerst, N., and Schuman, E.M. (2012). The local transcriptome in the synaptic neuropil revealed by deep sequencing and high-resolution imaging. Neuron 74, 453-466.

Candido, E.P., Reeves, R., and Davie, J.R. (1978). Sodium butyrate inhibits histone deacetylation in cultured cells. Cell 14, 105-113.

Cannon, W.B. (1929). Organization for Physiological Homeostasis. Physiol Rev 9, 399-431.

Carrasquillo, M.M., Belbin, O., Hunter, T.A., Ma, L., Bisceglio, G.D., Zou, F., Crook, J.E., Pankratz, V.S., Dickson, D.W., Graff-Radford, N.R., et al. (2010). Replication of CLU, CR1, and PICALM associations with alzheimer disease. Arch. Neurol. 67, 961-964.

Carrozza, M.J., Utley, R.T., Workman, J.L., and Côté, J. (2003). The diverse functions of histone acetyltransferase complexes. Trends in Genetics 19, 321-329.

De Carvalho Myskiw, J., Benetti, F., and Izquierdo, I. (2013). Behavioral tagging of extinction learning. Proc. Natl. Acad. Sci. U.S.A. 110, 1071-1076.

Castellucci, V.F., Frost, W.N., Goelet, P., Montarolo, P.G., Schacher, S., Morgan, J.A., Blumenfeld, H., and Kandel, E.R. (1986). Cell and molecular analysis of long-term sensitization in Aplysia. J. Physiol. (Paris) 81, 349-357.

Chen, Y.C., Gatchel, J.R., Lewis, R.W., Mao, C.-A., Grant, P.A., Zoghbi, H.Y., and Dent, S.Y.R. (2011). Gcn5 loss-offunction accelerates cerebellar and retinal degeneration in a SCA7 mouse model. Human Molecular Genetics.

Chepelev, I., Wei, G., Wangsa, D., Tang, Q., and Zhao, K. (2012). Characterization of genome-wide enhancerpromoter interactions reveals co-expression of interacting genes and modes of higher order chromatin organization. Cell Res. 22, 490-503.

Chibnik, L.B., Shulman, J.M., Leurgans, S.E., Schneider, J.A., Wilson, R.S., Tran, D., Aubin, C., Buchman, A.S., Heward, C.B., Myers, A.J., et al. (2011). CR1 is associated with amyloid plaque burden and age-related cognitive decline. Ann. Neurol. 69, 560-569.

Chwang, W.B., O'Riordan, K.J., Levenson, J.M., and Sweatt, J.D. (2006). ERK/MAPK regulates hippocampal histone phosphorylation following contextual fear conditioning. Learn. Mem. 13, 322-328.

Ciurciu, A., Komonyi, O., Pankotai, T., and Boros, I.M. (2006). The Drosophila histone acetyltransferase Gcn5 and transcriptional adaptor Ada2a are involved in nucleosomal histone H4 acetylation. Mol. Cell. Biol 26, 94139423.

Ciurciu, A., Komonyi, O., and Boros, I.M. (2008). Loss of ATAC-specific acetylation of histone H4 at Lys12 reduces binding of JIL-1 to chromatin and phosphorylation of histone H3 at Ser10. J. Cell. Sci 121, 3366-3372.

Corkin, S. (2002). What's new with the amnesic patient H.M.? Nat Rev Neurosci 3, 153-160. 
Coughenour, L.L., Mclean, J.R., and Parker, R.B. (1977). A new device for the rapid measurement of impaired motor function in mice. Pharmacol. Biochem. Behav. 6, 351-353.

Crehan, H., Holton, P., Wray, S., Pocock, J., Guerreiro, R., and Hardy, J. (2012). Complement receptor 1 (CR1) and Alzheimer's disease. Immunobiology 217, 244-250.

CSH Protocols (2007). Laemmli sample buffer (2X) with DTT. Cold Spring Harb Protoc 2007, pdb.rec10878.

Cui, H., Mason, B.L., Lee, C., Nishi, A., Elmquist, J.K., and Lutter, M. (2012). Melanocortin 4 receptor signaling in dopamine 1 receptor neurons is required for procedural memory learning. Physiol. Behav. 106, 201-210.

Czirr, E., and Wyss-Coray, T. (2012). The immunology of neurodegeneration. J. Clin. Invest. 122, 1156-1163.

Dai, J., Hyland, E.M., Yuan, D.S., Huang, H., Bader, J.S., and Boeke, J.D. (2008). Probing nucleosome function: a highly versatile library of synthetic histone H3 and H4 mutants. Cell 134, 1066-1078.

Danilova, A.B., and Grinkevich, L.N. (2012). Failure of long-term memory formation in juvenile snails is determined by acetylation status of histone $\mathrm{H} 3$ and can be improved by NaB treatment. PLoS ONE 7, e41828.

Dashwood, R.H., and Ho, E. (2008). Dietary agents as histone deacetylase inhibitors. Nutr Rev 66, S36-S38.

Davis, H.P., and Squire, L.R. (1984). Protein synthesis and memory: a review. Psychol Bull 96, 518-559.

Davoust, N., Nataf, S., Holers, V.M., and Barnum, S.R. (1999). Expression of the murine complement regulatory protein crry by glial cells and neurons. Glia 27, 162-170.

Deng, W., Aimone, J.B., and Gage, F.H. (2010). New neurons and new memories: how does adult hippocampal neurogenesis affect learning and memory? Nat Rev Neurosci 11, 339-350.

Dillman, A.A., Hauser, D.N., Gibbs, J.R., Nalls, M.A., McCoy, M.K., Rudenko, I.N., Galter, D., and Cookson, M.R. (2013). mRNA expression, splicing and editing in the embryonic and adult mouse cerebral cortex. Nat. Neurosci.

Dion, M.F., Altschuler, S.J., Wu, L.F., and Rando, O.J. (2005). Genomic characterization reveals a simple histone $\mathrm{H} 4$ acetylation code. Proceedings of the National Academy of Sciences of the United States of America 102, 5501-5506.

Dohm, C.P., Kermer, P., and Bähr, M. (2008). Aggregopathy in neurodegenerative diseases: mechanisms and therapeutic implication. Neurodegener Dis 5, 321-338.

Douglas, R.M., and Goddard, G.V. (1975). Long-term potentiation of the perforant path-granule cell synapse in the rat hippocampus. Brain Res. 86, 205-215.

Druz, A., Betenbaugh, M., and Shiloach, J. (2012). Glucose depletion activates mmu-miR-466h-5p expression through oxidative stress and inhibition of histone deacetylation. Nucleic Acids Res 40,7291-7302.

Dudai, Y. (2004). The neurobiology of consolidations, or, how stable is the engram? Annu Rev Psychol 55, 51-86.

Dudai, Y., and Eisenberg, M. (2004). Rites of passage of the engram: reconsolidation and the lingering consolidation hypothesis. Neuron 44, 93-100.

Durrin, L.K., Mann, R.K., Kayne, P.S., and Grunstein, M. (1991). Yeast histone H4 N-terminal sequence is required for promoter activation in vivo. Cell 65, 1023-1031.

Eichenbaum, H. (1997). How does the brain organize memories? Science 277, 330-332.

Ekwall, K. (2005). Genome-wide analysis of HDAC function. Trends in Genetics 21, 608-615.

Elfving, B., Bonefeld, B.E., Rosenberg, R., and Wegener, G. (2008). Differential expression of synaptic vesicle proteins after repeated electroconvulsive seizures in rat frontal cortex and hippocampus. Synapse 62, 662-670.

Erickson, K.I., Voss, M.W., Prakash, R.S., Basak, C., Szabo, A., Chaddock, L., Kim, J.S., Heo, S., Alves, H., White, S.M., et al. (2011). Exercise training increases size of hippocampus and improves memory. PNAS.

Federman, N., Fustiñana, M.S., and Romano, A. (2009). Histone acetylation is recruited in consolidation as a molecular feature of stronger memories. Learn. Mem 16, 600-606. 
Fendt, M., and Fanselow, M.S. (1999). The neuroanatomical and neurochemical basis of conditioned fear. Neurosci Biobehav Rev 23, 743-760.

Feser, J., Truong, D., Das, C., Carson, J.J., Kieft, J., Harkness, T., and Tyler, J.K. (2010). Elevated Histone Expression Promotes Life Span Extension. Molecular Cell 39, 724-735.

Finch, C.E., and Morgan, D.G. (1990). RNA and protein metabolism in the aging brain. Annu. Rev. Neurosci. 13, 75-88.

Fischer, A. (2010). HDAC-Inhibitoren als Therapie für neuronale Erkrankungen. Entdeckung neuer Anwendungsgebiete. Pharmazie in Unserer Zeit 39, 204-209.

Fischer, A., Sananbenesi, F., Wang, X., Dobbin, M., and Tsai, L.-H. (2007). Recovery of learning and memory is associated with chromatin remodelling. Nature $447,178-182$.

Fitts, P.M. (1954). The information capacity of the human motor system in controlling the amplitude of movement. J Exp Psychol 47, 381-391.

Fitzsimons, H.L., and Scott, M.J. (2011). Genetic modulation of Rpd3 expression impairs long-term courtship memory in Drosophila. PLoS ONE 6, e29171.

Flexner, J.B., Flexner, L.B., and Stellar, E. (1963). Memory in mice as affected by intracerebral puromycin. Science $141,57-59$.

Fonseca, M.I., Zhou, J., Botto, M., and Tenner, A.J. (2004). Absence of C1q leads to less neuropathology in transgenic mouse models of Alzheimer's disease. J. Neurosci. 24, 6457-6465.

Fontán-Lozano, A., Romero-Granados, R., Troncoso, J., Múnera, A., Delgado-García, J.M., and Carrión, A.M. (2008). Histone deacetylase inhibitors improve learning consolidation in young and in KA-inducedneurodegeneration and SAMP-8-mutant mice. Mol. Cell. Neurosci. 39, 193-201.

Fox, A.H., Lam, Y.W., Leung, A.K.L., Lyon, C.E., Andersen, J., Mann, M., and Lamond, A.I. (2002). Paraspeckles: a novel nuclear domain. Curr. Biol. 12,13-25.

Francis, Y.I., Fà, M., Ashraf, H., Zhang, H., Staniszewski, A., Latchman, D.S., and Arancio, O. (2009). Dysregulation of histone acetylation in the APP/PS1 mouse model of Alzheimer's disease. J. Alzheimers Dis 18, 131-139.

Frey, U., and Morris, R.G. (1997). Synaptic tagging and long-term potentiation. Nature 385, 533-536.

Garner, A.R., Rowland, D.C., Hwang, S.Y., Baumgaertel, K., Roth, B.L., Kentros, C., and Mayford, M. (2012). Generation of a Synthetic Memory Trace. Science 335, 1513-1516.

Gaub, P., Tedeschi, A., Puttagunta, R., Nguyen, T., Schmandke, A., and Di Giovanni, S. (2010). HDAC inhibition promotes neuronal outgrowth and counteracts growth cone collapse through CBP/p300 and P/CAF-dependent p53 acetylation. Cell Death Differ 17, 1392-1408.

Gazzaniga, M.S. (2004). The Cognitive Neurosciences (MIT Press).

Gentleman, R.C., Carey, V.J., Bates, D.M., Bolstad, B., Dettling, M., Dudoit, S., Ellis, B., Gautier, L., Ge, Y., Gentry, J., et al. (2004). Bioconductor: open software development for computational biology and bioinformatics. Genome Biol. 5, R80.

Ginsburg, D.S., Govind, C.K., and Hinnebusch, A.G. (2009). NuA4 lysine acetyltransferase Esa1 is targeted to coding regions and stimulates transcription elongation with Gcn5. Mol. Cell. Biol 29, 6473-6487.

Goddyn, H., Leo, S., Meert, T., and D'Hooge, R. (2006). Differences in behavioural test battery performance between mice with hippocampal and cerebellar lesions. Behavioural Brain Research 173, 138-147.

Govindarajan, N., Agis-Balboa, R.C., Walter, J., Sananbenesi, F., and Fischer, A. (2011). Sodium butyrate improves memory function in an Alzheimer's disease mouse model when administered at an advanced stage of disease progression. J. Alzheimers Dis. 26, 187-197.

Govindarajan, N., Rao, P., Burkhardt, S., Sananbenesi, F., Schlüter, O.M., Bradke, F., Lu, J., and Fischer, A. (2012). Reducing HDAC6 ameliorates cognitive deficits in a mouse model for Alzheimer's disease. EMBO Mol Med. 
Gräff, J., and Tsai, L.-H. (2013a). Histone acetylation: molecular mnemonics on the chromatin. Nature Reviews Neuroscience $14,97-111$.

Gräff, J., and Tsai, L.-H. (2013b). The potential of HDAC inhibitors as cognitive enhancers. Annu. Rev. Pharmacol. Toxicol. 53, 311-330.

Gray, D.A., and Woulfe, J. (2005). Lipofuscin and aging: a matter of toxic waste. Sci Aging Knowledge Environ 2005, re1.

Grinkevich, L.N. (2012). [Investigation of histone H3 methylation during long term memory formation]. Ross Fiziol Zh Im I M Sechenova 98, 1111-1118.

Guan, J.-S., Haggarty, S.J., Giacometti, E., Dannenberg, J.-H., Joseph, N., Gao, J., Nieland, T.J.F., Zhou, Y., Wang, X., Mazitschek, R., et al. (2009). HDAC2 negatively regulates memory formation and synaptic plasticity. Nature $459,55-60$.

Guelman, S., Kozuka, K., Mao, Y., Pham, V., Solloway, M.J., Wang, J., Wu, J., Lill, J.R., and Zha, J. (2009). The Double-Histone-Acetyltransferase Complex ATAC Is Essential for Mammalian Development. Mol. Cell. Biol. 29, 1176-1188.

Gunderson, F.Q., and Johnson, T.L. (2009). Acetylation by the Transcriptional Coactivator Gcn5 Plays a Novel Role in Co-Transcriptional Spliceosome Assembly. PLoS Genet 5, e1000682.

Gunesdogan, U., Jackle, H., and Herzig, A. (2010). A genetic system to assess in vivo the functions of histones and histone modifications in higher eukaryotes. EMBO Rep 11,772-776.

Gupta, S., Kim, S.Y., Artis, S., Molfese, D.L., Schumacher, A., Sweatt, J.D., Paylor, R.E., and Lubin, F.D. (2010). Histone Methylation Regulates Memory Formation. J. Neurosci. 30, 3589-3599.

Haettig, J., Stefanko, D.P., Multani, M.L., Figueroa, D.X., McQuown, S.C., and Wood, M.A. (2011). HDAC inhibition modulates hippocampus-dependent long-term memory for object location in a CBP-dependent manner. Learning \& Memory 18, 71-79.

Hagihara, H., Toyama, K., Yamasaki, N., and Miyakawa, T. (2009). Dissection of Hippocampal Dentate Gyrus from Adult Mouse. Journal of Visualized Experiments.

Hait, N.C., Allegood, J., Maceyka, M., Strub, G.M., Harikumar, K.B., Singh, S.K., Luo, C., Marmorstein, R., Kordula, T., Milstien, S., et al. (2009). Regulation of histone acetylation in the nucleus by sphingosine-1-phosphate. Science $325,1254-1257$.

Hargreaves, D.C., Horng, T., and Medzhitov, R. (2009). Control of Inducible Gene Expression by SignalDependent Transcriptional Elongation. Cell 138, 129-145.

Harries, L.W., Hernandez, D., Henley, W., Wood, A., Holly, A.C., Bradley-Smith, R.M., Yaghootkar, H., Dutta, A., Murray, A., Frayling, T.M., et al. (2011). Human aging is characterized by focused changes in gene expression and deregulation of alternative splicing. Aging Cell.

Hassan, A.H., Awad, S., Al-Natour, Z., Othman, S., Mustafa, F., and Rizvi, T.A. (2007). Selective recognition of acetylated histones by bromodomains in transcriptional co-activators. Biochem. J. 402, 125.

Hazrati, L.-N., Van Cauwenberghe, C., Brooks, P.L., Brouwers, N., Ghani, M., Sato, C., Cruts, M., Sleegers, K., St George-Hyslop, P., Van Broeckhoven, C., et al. (2012). Genetic association of CR1 with Alzheimer's disease: a tentative disease mechanism. Neurobiol. Aging 33, 2949.e5-2949.e12.

Hedden, T., and Gabrieli, J.D.E. (2004). Insights into the ageing mind: a view from cognitive neuroscience. Nat Rev Neurosci 5, 87-96.

Herrera, J.E., Bergel, M., Yang, X.-J., Nakatani, Y., and Bustin, M. (1997). The Histone Acetyltransferase Activity of Human GCN5 and PCAF Is Stabilized by Coenzymes. J. Biol. Chem. 272, 27253-27258.

Holliday, R. (2006). Epigenetics: a historical overview. Epigenetics 1, 76-80.

Hörtnagl, H., Berger, M.L., Sperk, G., and Pifl, C. (1991). Regional heterogeneity in the distribution of neurotransmitter markers in the rat hippocampus. Neuroscience 45, 261-272. 
Huang, D.W., Sherman, B.T., and Lempicki, R.A. (2009a). Systematic and integrative analysis of large gene lists using DAVID bioinformatics resources. Nat Protoc 4, 44-57.

Huang, D.W., Sherman, B.T., and Lempicki, R.A. (2009b). Bioinformatics enrichment tools: paths toward the comprehensive functional analysis of large gene lists. Nucleic Acids Res. 37, 1-13.

Huang, W.C., Xiao, S., Huang, F., Harfe, B.D., Jan, Y.N., and Jan, L.Y. (2012). Calcium-activated chloride channels (CaCCs) regulate action potential and synaptic response in hippocampal neurons. Neuron 74, 179-192.

Huber, W., Von Heydebreck, A., Sültmann, H., Poustka, A., and Vingron, M. (2002). Variance stabilization applied to microarray data calibration and to the quantification of differential expression. Bioinformatics 18 Suppl 1 , S96-104.

Igaz, L.M., Vianna, M.R.M., Medina, J.H., and Izquierdo, I. (2002). Two time periods of hippocampal mRNA synthesis are required for memory consolidation of fear-motivated learning. J. Neurosci. 22, 6781-6789.

Igaz, L.M., Bekinschtein, P., Vianna, M.M.R., Izquierdo, I., and Medina, J.H. (2004). Gene expression during memory formation. Neurotox Res 6, 189-204.

Iordanova, M.D., Burnett, D.J., Aggleton, J.P., Good, M., and Honey, R.C. (2009). The role of the hippocampus in mnemonic integration and retrieval: complementary evidence from lesion and inactivation studies. Eur. J. Neurosci. 30, 2177-2189.

Jacobs, L.F., Gaulin, S.J., Sherry, D.F., and Hoffman, G.E. (1990). Evolution of spatial cognition: sex-specific patterns of spatial behavior predict hippocampal size. PNAS 87, 6349-6352.

Jacobson, A.C., and Weis, J.H. (2008). Comparative Functional Evolution of Human and Mouse CR1 and CR2. J Immunol 181, 2953-2959.

Jang, S., Dilger, R.N., and Johnson, R.W. (2010). Luteolin Inhibits Microglia and Alters Hippocampal-Dependent Spatial Working Memory in Aged Mice. Journal of Nutrition.

Ji, J., and Maren, S. (2008). Differential roles for hippocampal areas CA1 and CA3 in the contextual encoding and retrieval of extinguished fear. Learn. Mem. 15, 244-251.

Johnsson, A.E., and Wright, A.P.H. (2010). The role of specific HAT-HDAC interactions in transcriptional elongation. Cell Cycle 9, 467-471.

Johnsson, A., Durand-Dubief, M., Xue-Franzén, Y., Rönnerblad, M., Ekwall, K., and Wright, A. (2009). HAT-HDAC interplay modulates global histone H3K14 acetylation in gene-coding regions during stress. EMBO Rep 10, 1009-1014.

Jones, B.C., Hou, X., and Cook, M.N. (1996). Effect of exposure to novelty on brain monoamines in C57BL/6 and DBA/2 mice. Physiol. Behav. 59, 361-367.

Kanfi, Y., Naiman, S., Amir, G., Peshti, V., Zinman, G., Nahum, L., Bar-Joseph, Z., and Cohen, H.Y. (2012). The sirtuin SIRT6 regulates lifespan in male mice. Nature 483, 218-221.

Kanno, T., Kanno, Y., Siegel, R.M., Jang, M.K., Lenardo, M.J., and Ozato, K. (2004). Selective recognition of acetylated histones by bromodomain proteins visualized in living cells. Mol. Cell 13, 33-43.

Keenan, B.T., Shulman, J.M., Chibnik, L.B., Raj, T., Tran, D., Sabuncu, M.R., Allen, A.N., Corneveaux, J.J., Hardy, J.A., Huentelman, M.J., et al. (2012). A coding variant in CR1 interacts with APOE- 44 to influence cognitive decline. Hum. Mol. Genet. 21, 2377-2388.

Kerimoglu, C. (2012). Role of Histone Methylation in Cognition and Effects of Different Durations of Environmental Enrichment on Learning and Memory.

Kerimoglu, C., Agis-Balboa, R.C., Kranz, A., Stilling, R., Bahari-Javan, S., Benito-Garagorri, E., Halder, R., Burkhardt, S., Stewart, A.F., and Fischer, A. (2013). Histone-Methyltransferase MLL2 (KMT2B) Is Required for Memory Formation in Mice. J. Neurosci. 33, 3452-3464.

Kerman, I.A., Clinton, S.M., Bedrosian, T.A., Abraham, A.D., Rosenthal, D.T., Akil, H., and Watson, S.J. (2011). High novelty-seeking predicts aggression and gene expression differences within defined serotonergic cell groups. Brain Res. 1419, 34-45. 
Kilgore, M., Miller, C.A., Fass, D.M., Hennig, K.M., Haggarty, S.J., Sweatt, J.D., and Rumbaugh, G. (2009). Inhibitors of Class 1 Histone Deacetylases Reverse Contextual Memory Deficits in a Mouse Model of Alzheimer's Disease. Neuropsychopharmacology 35, 870-880.

Kim, J.J., and Fanselow, M.S. (1992). Modality-specific retrograde amnesia of fear. Science 256, 675-677.

Kim, J.-H., Lane, W.S., and Reinberg, D. (2002). Human Elongator facilitates RNA polymerase II transcription through chromatin. Proceedings of the National Academy of Sciences of the United States of America 99 , 1241-1246.

King, M.V., Marsden, C.A., and Fone, K.C.F. (2008). A role for the 5-HT(1A), 5-HT4 and 5-HT6 receptors in learning and memory. Trends Pharmacol. Sci. 29, 482-492.

Kohman, R.A., Rodriguez-Zas, S.L., Southey, B.R., Kelley, K.W., Dantzer, R., and Rhodes, J.S. (2011). Voluntary Wheel Running Reverses Age-Induced Changes in Hippocampal Gene Expression. PLoS ONE 6, e22654.

Kojima, T., Matsumoto, M., Togashi, H., Tachibana, K., Kemmotsu, O., and Yoshioka, M. (2003). Fluvoxamine suppresses the long-term potentiation in the hippocampal CA1 field of anesthetized rats: an effect mediated via 5-HT1A receptors. Brain Res. 959, 165-168.

Kosik, K.S., Rapp, P.R., Raz, N., Small, S.A., Sweatt, J.D., and Tsai, L.-H. (2012). Mechanisms of Age-Related Cognitive Change and Targets for Intervention: Epigenetics. J Gerontol A Biol Sci Med Sci.

Kouzarides, T. (2007). Chromatin Modifications and Their Function. Cell 128, 693-705.

Krebs, A.R., Karmodiya, K., Lindahl-Allen, M., Struhl, K., and Tora, L. (2011). SAGA and ATAC histone acetyl transferase complexes regulate distinct sets of genes and ATAC defines a class of p300-independent enhancers. Mol. Cell 44, 410-423.

Krych-Goldberg, M., and Atkinson, J.P. (2001). Structure-function relationships of complement receptor type 1. Immunol. Rev. 180, 112-122.

Kuczera, T., Stilling, R.M., Hsia, H.-E., Bahari-Javan, S., Irniger, S., Nasmyth, K., Sananbenesi, F., and Fischer, A. (2011). The anaphase promoting complex is required for memory function in mice. Learn. Mem 18, 49-57.

Kulkarni, A.P., Pillay, N.S., Kellaway, L.A., and Kotwal, G.J. (2008). Intracranial administration of vaccinia virus complement control protein in Mo/Hu APPswe PS1dE9 transgenic mice at an early age shows enhanced performance at a later age using a cheese board maze test. Biogerontology 9, 405-420.

Kulkarni, A.P., Govender, D.A., Kotwal, G.J., and Kellaway, L.A. (2011). Modulation of anxiety behavior by intranasally administered vaccinia virus complement control protein and curcumin in a mouse model of Alzheimer's disease. Curr Alzheimer Res 8, 95-113.

Ladurner, A.G. (2006). Rheostat control of gene expression by metabolites. Mol. Cell 24, 1-11.

Lah, S., and Miller, L. (2008). Effects of temporal lobe lesions on retrograde memory: a critical review. Neuropsychol Rev 18, 24-52.

Lambert, J.-C., Heath, S., Even, G., Campion, D., Sleegers, K., Hiltunen, M., Combarros, O., Zelenika, D., Bullido, M.J., Tavernier, B., et al. (2009). Genome-wide association study identifies variants at CLU and CR1 associated with Alzheimer's disease. Nature Genetics 41, 1094-1099.

Langmead, B., and Salzberg, S.L. (2012). Fast gapped-read alignment with Bowtie 2. Nature Methods 9, 357359

Latham, T., Mackay, L., Sproul, D., Karim, M., Culley, J., Harrison, D.J., Hayward, L., Langridge-Smith, P., Gilbert, N., and Ramsahoye, B.H. (2012). Lactate, a product of glycolytic metabolism, inhibits histone deacetylase activity and promotes changes in gene expression. Nucl. Acids Res. 40, 4794-4803.

Lavenex, P., and Amaral, D.G. (2000). Hippocampal-neocortical interaction: a hierarchy of associativity. Hippocampus 10, 420-430.

Lechner, H.A., Squire, L.R., and Byrne, J.H. (1999). 100 years of consolidation--remembering Müller and Pilzecker. Learn. Mem. 6, 77-87. 
Lein, E.S., Hawrylycz, M.J., Ao, N., Ayres, M., Bensinger, A., Bernard, A., Boe, A.F., Boguski, M.S., Brockway, K.S., Byrnes, E.J., et al. (2007). Genome-wide atlas of gene expression in the adult mouse brain. Nature 445, 168176.

Leinhase, I., Schmidt, O.I., Thurman, J.M., Hossini, A.M., Rozanski, M., Taha, M.E., Scheffler, A., John, T., Smith, W.R., Holers, V.M., et al. (2006). Pharmacological complement inhibition at the C3 convertase level promotes neuronal survival, neuroprotective intracerebral gene expression, and neurological outcome after traumatic brain injury. Exp. Neurol. 199, 454-464.

LeRoy, G., Rickards, B., and Flint, S.J. (2008). The double bromodomain proteins Brd2 and Brd3 couple histone acetylation to transcription. Mol. Cell 30, 51-60.

Levenson, J.M., O’Riordan, K.J., Brown, K.D., Trinh, M.A., Molfese, D.L., and Sweatt, J.D. (2004). Regulation of Histone Acetylation during Memory Formation in the Hippocampus. Journal of Biological Chemistry 279, 40545-40559.

Li, H., Handsaker, B., Wysoker, A., Fennell, T., Ruan, J., Homer, N., Marth, G., Abecasis, G., and Durbin, R. (2009a). The Sequence Alignment/Map format and SAMtools. Bioinformatics 25, 2078-2079.

Li, Q., Lee, J.-A., and Black, D.L. (2007). Neuronal regulation of alternative pre-mRNA splicing. Nat Rev Neurosci 8, 819-831.

Li, Z., Wright, F.A., and Royland, J. (2009b). Age-dependent variability in gene expression in male Fischer 344 rat retina. Toxicol. Sci. 107, 281-292.

Lin, W., Zhang, Z., Srajer, G., Chen, Y.C., Huang, M., Phan, H.M., and Dent, S.Y.R. (2008). Proper expression of the Gcn5 histone acetyltransferase is required for neural tube closure in mouse embryos. Developmental Dynamics $237,928-940$.

Liu, X., Ramirez, S., Pang, P.T., Puryear, C.B., Govindarajan, A., Deisseroth, K., and Tonegawa, S. (2012). Optogenetic stimulation of a hippocampal engram activates fear memory recall. Nature.

Loeffler, D.A. (2004). Using animal models to determine the significance of complement activation in Alzheimer's disease. Journal of Neuroinflammation 1, 18.

Loerch, P.M., Lu, T., Dakin, K.A., Vann, J.M., Isaacs, A., Geula, C., Wang, J., Pan, Y., Gabuzda, D.H., Li, C., et al. (2008). Evolution of the Aging Brain Transcriptome and Synaptic Regulation. PLoS ONE 3, e3329.

Lømo, T. (2003). The discovery of long-term potentiation. Philos Trans R Soc Lond B Biol Sci 358, 617-620.

Lopez, O.L. (2011). The growing burden of Alzheimer's disease. Am J Manag Care 17 Suppl 13, S339-345.

Lu, T., Pan, Y., Kao, S.-Y., Li, C., Kohane, I., Chan, J., and Yankner, B.A. (2004). Gene regulation and DNA damage in the ageing human brain. Nature 429, 883-891.

Maguire, E.A., Woollett, K., and Spiers, H.J. (2006). London taxi drivers and bus drivers: a structural MRI and neuropsychological analysis. Hippocampus 16, 1091-1101.

Maier, M., Peng, Y., Jiang, L., Seabrook, T.J., Carroll, M.C., and Lemere, C.A. (2008). Complement C3 deficiency leads to accelerated amyloid beta plaque deposition and neurodegeneration and modulation of the microglia/macrophage phenotype in amyloid precursor protein transgenic mice. J. Neurosci. 28, 6333-6341.

Mann, B.S., Johnson, J.R., Cohen, M.H., Justice, R., and Pazdur, R. (2007). FDA approval summary: vorinostat for treatment of advanced primary cutaneous T-cell lymphoma. Oncologist 12, 1247-1252.

Martin, S.J., Grimwood, P.D., and Morris, R.G. (2000). Synaptic plasticity and memory: an evaluation of the hypothesis. Annu. Rev. Neurosci. 23, 649-711.

Martinez, E., Palhan, V.B., Tjernberg, A., Lymar, E.S., Gamper, A.M., Kundu, T.K., Chait, B.T., and Roeder, R.G. (2001). Human STAGA complex is a chromatin-acetylating transcription coactivator that interacts with premRNA splicing and DNA damage-binding factors in vivo. Mol. Cell. Biol 21, 6782-6795.

Martínez-Cerdeño, V., Lemen, J.M., Chan, V., Wey, A., Lin, W., Dent, S.R., and Knoepfler, P.S. (2012). N-Myc and GCN5 Regulate Significantly Overlapping Transcriptional Programs in Neural Stem Cells. PLoS ONE 7, e39456. 
Maurice, T., Duclot, F., Meunier, J., Naert, G., Givalois, L., Meffre, J., Célérier, A., Jacquet, C., Copois, V., Mechti, N., et al. (2008). Altered Memory Capacities and Response to Stress in p300/CBP-Associated Factor (PCAF) Histone Acetylase Knockout Mice. Neuropsychopharmacology 33, 1584-1602.

Mayford, M., Bach, M.E., Huang, Y.-Y., Wang, L., Hawkins, R.D., and Kandel, E.R. (1996). Control of Memory Formation Through Regulated Expression of a CaMKII Transgene. Science 274, 1678-1683.

Mazin, P., Xiong, J., Liu, X., Yan, Z., Zhang, X., Li, M., He, L., Somel, M., Yuan, Y., Phoebe Chen, Y.-P., et al. (2013). Widespread splicing changes in human brain development and aging. Mol. Syst. Biol. 9, 633.

McGaugh, J.L. (2000). Memory--a century of consolidation. Science 287, 248-251.

McHugh, M.L. (2011). Multiple comparison analysis testing in ANOVA. Biochem Med (Zagreb) 21, $203-209$.

McQuown, S.C., and Wood, M.A. (2011). HDAC3 and the Molecular Brake Pad Hypothesis. Neurobiol Learn Mem $96,27-34$.

Merschbaecher, K., Haettig, J., and Mueller, U. (2012). Acetylation-mediated suppression of transcriptionindependent memory: bidirectional modulation of memory by acetylation. PLoS ONE 7, e45131.

Meshorer, E., and Soreq, H. (2002). Pre-mRNA splicing modulations in senescence. Aging Cell 1, 10-16.

Michan, S., and Sinclair, D. (2007). Sirtuins in mammals: insights into their biological function. Biochem. J. 404, $1-13$.

Miller, C.A., and Sweatt, J.D. (2007). Covalent modification of DNA regulates memory formation. Neuron 53, 857-869.

Miller, C.A., Campbell, S.L., and Sweatt, J.D. (2008). DNA methylation and histone acetylation work in concert to regulate memory formation and synaptic plasticity. Neurobiol Learn Mem 89, 599-603.

Minichiello, L., Korte, M., Wolfer, D., Kühn, R., Unsicker, K., Cestari, V., Rossi-Arnaud, C., Lipp, H.-P., Bonhoeffer, T., and Klein, R. (1999). Essential Role for TrkB Receptors in Hippocampus-Mediated Learning. Neuron 24, 401414.

Moncada, D., and Viola, H. (2007). Induction of long-term memory by exposure to novelty requires protein synthesis: evidence for a behavioral tagging. J. Neurosci. 27, 7476-7481.

Moncada, D., Ballarini, F., Martinez, M.C., Frey, J.U., and Viola, H. (2011). Identification of transmitter systems and learning tag molecules involved in behavioral tagging during memory formation. Proc. Natl. Acad. Sci. U.S.A. $108,12931-12936$.

Montarolo, P.G., Goelet, P., Castellucci, V.F., Morgan, J., Kandel, E.R., and Schacher, S. (1986). A critical period for macromolecular synthesis in long-term heterosynaptic facilitation in Aplysia. Science 234, 1249-1254.

Morgan, J.I., and Curran, T. (1988). Calcium as a modulator of the immediate-early gene cascade in neurons. Cell Calcium 9, 303-311.

Morgan, J.I., and Curran, T. (1989). Stimulus-transcription coupling in neurons: role of cellular immediate-early genes. Trends Neurosci. 12, 459-462.

Mori, K., Togashi, H., Kojima, T., Matsumoto, M., Ohashi, S., Ueno, K., and Yoshioka, M. (2001). Different effects of anxiolytic agents, diazepam and 5-HT(1A) agonist tandospirone, on hippocampal long-term potentiation in vivo. Pharmacol. Biochem. Behav. 69, 367-372.

Moriyama, M., Fukuhara, T., Britschgi, M., He, Y., Narasimhan, R., Villeda, S., Molina, H., Huber, B.T., Holers, M., and Wyss-Coray, T. (2011). Complement receptor 2 is expressed in neural progenitor cells and regulates adult hippocampal neurogenesis. J. Neurosci. 31, 3981-3989.

Moroz, L.L., and Kohn, A.B. (2010). Do different neurons age differently? Direct genome-wide analysis of aging in single identified cholinergic neurons. Front Aging Neurosci 2.

Morris, R. (1984). Developments of a water-maze procedure for studying spatial learning in the rat. Journal of Neuroscience Methods 11, 47-60. 
Moser, M.B., and Moser, E.I. (1998). Functional differentiation in the hippocampus. Hippocampus 8, 608-619.

Muller, F.L., Lustgarten, M.S., Jang, Y., Richardson, A., and Van Remmen, H. (2007). Trends in oxidative aging theories. Free Radic. Biol. Med. 43, 477-503.

Müller, G., and Pilzecker, A. (1900). Experimentelle Beiträge zur Lehre vom Gedächtnis. Z. Psychol. Ergänzungsband 1, 1-300.

Muratore, C.R., Hodgson, N.W., Trivedi, M.S., Abdolmaleky, H.M., Persico, A.M., Lintas, C., De La Monte, S., and Deth, R.C. (2013). Age-dependent decrease and alternative splicing of methionine synthase mRNA in human cerebral cortex and an accelerated decrease in autism. PLoS ONE 8, e56927.

Murray, A. (1961). Labeling of biologically important compounds with radioisotopes. Los Alamos Sci LAMS-2526, 248.

Naganuma, T., and Hirose, T. (2013). Paraspeckle formation during the biogenesis of long noncoding RNAs. RNA Biol 10.

Nagy, Z., and Tora, L. (2007). Distinct GCN5/PCAF-containing complexes function as co-activators and are involved in transcription factor and global histone acetylation. Oncogene 26, 5341-5357.

Nagy, Z., Riss, A., Fujiyama, S., Krebs, A., Orpinell, M., Jansen, P., Cohen, A., Stunnenberg, H.G., Kato, S., and Tora, L. (2010). The metazoan ATAC and SAGA coactivator HAT complexes regulate different sets of inducible target genes. Cell. Mol. Life Sci. 67, 611-628.

Nguyen, P.V., Abel, T., and Kandel, E.R. (1994). Requirement of a critical period of transcription for induction of a late phase of LTP. Science $265,1104-1107$.

Nitsche, M.A., Schauenburg, A., Lang, N., Liebetanz, D., Exner, C., Paulus, W., and Tergau, F. (2003). Facilitation of implicit motor learning by weak transcranial direct current stimulation of the primary motor cortex in the human. J Cogn Neurosci 15, 619-626.

O'Sullivan, R.J., and Karlseder, J. (2012). The great unravelling: chromatin as a modulator of the aging process. Trends Biochem. Sci. 37, 466-476.

O'Sullivan, R.J., Kubicek, S., Schreiber, S.L., and Karlseder, J. (2010). Reduced histone biosynthesis and chromatin changes arising from a damage signal at telomeres. Nat. Struct. Mol. Biol. 17, 1218-1225.

Oberdoerffer, P., Michan, S., McVay, M., Mostoslavsky, R., Vann, J., Park, S.-K., Hartlerode, A., Stegmuller, J., Hafner, A., Loerch, P., et al. (2008). SIRT1 redistribution on chromatin promotes genomic stability but alters gene expression during aging. Cell 135, 907-918.

Ogren, S.O., Eriksson, T.M., Elvander-Tottie, E., D'Addario, C., Ekström, J.C., Svenningsson, P., Meister, B., Kehr, J., and Stiedl, O. (2008). The role of 5-HT(1A) receptors in learning and memory. Behav. Brain Res. 195, 54-77.

Osorio, F.G., Navarro, C.L., Cadiñanos, J., López-Mejía, I.C., Quirós, P.M., Bartoli, C., Rivera, J., Tazi, J., Guzmán, G., Varela, I., et al. (2011). Splicing-Directed Therapy in a New Mouse Model of Human Accelerated Aging. Science Translational Medicine 3, 106 ra107.

Owen, D.J., Ornaghi, P., Yang, J.-C., Lowe, N., Evans, P.R., Ballario, P., Neuhaus, D., Filetici, P., and Travers, A.A. (2000). The structural basis for the recognition of acetylated histone $\mathrm{H} 4$ by the bromodomain of histone acetyltransferase Gcn5p. EMBO J 19, 6141-6149.

Patel, J.H., Du, Y., Ard, P.G., Phillips, C., Carella, B., Chen, C.-J., Rakowski, C., Chatterjee, C., Lieberman, P.M., Lane, W.S., et al. (2004). The c-MYC oncoprotein is a substrate of the acetyltransferases hGCN5/PCAF and TIP60. Mol. Cell. Biol. 24, 10826-10834.

Pawlowski, T.L., Bellush, L.L., Wright, A.W., Walker, J.P., Colvin, R.A., and Huentelman, M.J. (2009). Hippocampal gene expression changes during age-related cognitive decline. Brain Res. 1256, 101-110.

Peleg, S., Sananbenesi, F., Zovoilis, A., Burkhardt, S., Bahari-Javan, S., Agis-Balboa, R.C., Cota, P., Wittnam, J.L., Gogol-Doering, A., Opitz, L., et al. (2010). Altered histone acetylation is associated with age-dependent memory impairment in mice. Science $328,753-756$.

Pengelly, A.R., Copur, Ö., Jäckle, H., Herzig, A., and Müller, J. (2013). A Histone Mutant Reproduces the Phenotype Caused by Loss of Histone-Modifying Factor Polycomb. Science 339, 698-699. 
Penner, M.R., Roth, T.L., Chawla, M.K., Hoang, L.T., Roth, E.D., Lubin, F.D., Sweatt, J.D., Worley, P.F., and Barnes, C.A. (2010a). Age-related changes in Arc transcription and DNA methylation within the hippocampus. Neurobiol. Aging.

Penner, M.R., Roth, T.L., Barnes, C.A., and Sweatt, J.D. (2010b). An epigenetic hypothesis of aging-related cognitive dysfunction. Front Aging Neurosci 2, 9.

Pillay, N.S., Kellaway, L.A., and Kotwal, G.J. (2008). Early detection of memory deficits and memory improvement with vaccinia virus complement control protein in an Alzheimer's disease model. Behav. Brain Res. 192, 173-177.

Pletcher, S.D., Macdonald, S.J., Marguerie, R., Certa, U., Stearns, S.C., Goldstein, D.B., and Partridge, L. (2002). Genome-wide transcript profiles in aging and calorically restricted Drosophila melanogaster. Curr. Biol. 12, 712-723.

Praag, H. van, Shubert, T., Zhao, C., and Gage, F.H. (2005). Exercise Enhances Learning and Hippocampal Neurogenesis in Aged Mice. J. Neurosci. 25, 8680-8685.

Van Praag, H., Christie, B.R., Sejnowski, T.J., and Gage, F.H. (1999). Running enhances neurogenesis, learning, and long-term potentiation in mice. Proc. Natl. Acad. Sci. U.S.A. 96, 13427-13431.

Rahman, I., Marwick, J., and Kirkham, P. (2004). Redox modulation of chromatin remodeling: impact on histone acetylation and deacetylation, NF-kappaB and pro-inflammatory gene expression. Biochem. Pharmacol 68, 1255-1267.

Ramaglia, V., Hughes, T.R., Donev, R.M., Ruseva, M.M., Wu, X., Huitinga, I., Baas, F., Neal, J.W., and Morgan, B.P. (2012). C3-Dependent Mechanism of Microglial Priming Relevant to Multiple Sclerosis. PNAS 109, 965-970.

Rancan, M., Morganti-Kossmann, M.C., Barnum, S.R., Saft, S., Schmidt, O.I., Ertel, W., and Stahel, P.F. (2003). Central nervous system-targeted complement inhibition mediates neuroprotection after closed head injury in transgenic mice. J. Cereb. Blood Flow Metab. 23, 1070-1074.

Raz, N., and Rodrigue, K.M. (2006). Differential aging of the brain: Patterns, cognitive correlates and modifiers. Neuroscience \& Biobehavioral Reviews 30, 730-748.

Reichwald, J., Danner, S., Wiederhold, K.-H., and Staufenbiel, M. (2009). Expression of complement system components during aging and amyloid deposition in APP transgenic mice. Journal of Neuroinflammation 6, 35.

Reijmers, L.G., Perkins, B.L., Matsuo, N., and Mayford, M. (2007). Localization of a stable neural correlate of associative memory. Science 317, 1230-1233.

Reymann, K.G., and Frey, J.U. (2007). The late maintenance of hippocampal LTP: requirements, phases, "synaptic tagging", "late-associativity" and implications. Neuropharmacology 52, 24-40.

Ricobaraza, A., Cuadrado-Tejedor, M., Pérez-Mediavilla, A., Frechilla, D., Del Río, J., and García-Osta, A. (2009). Phenylbutyrate ameliorates cognitive deficit and reduces tau pathology in an Alzheimer's disease mouse model. Neuropsychopharmacology 34,1721-1732.

Rodier, F., Campisi, J., and Bhaumik, D. (2007). Two faces of p53: aging and tumor suppression. Nucleic Acids Res 35, 7475-7484.

Romcy-Pereira, R.N., Erraji-Benchekroun, L., Smyrniotopoulos, P., Ogawa, S., Mello, C.V., Sibille, E., and Pavlides, C. (2009). Sleep-Dependent Gene Expression in the Hippocampus and Prefrontal Cortex Following Long-Term Potentiation. Physiol Behav 98, 44-52.

Rotenberg, A., Mayford, M., Hawkins, R.D., Kandel, E.R., and Muller, R.U. (1996). Mice expressing activated CaMKII lack low frequency LTP and do not form stable place cells in the CA1 region of the hippocampus. Cell $87,1351-1361$.

Roth, S.Y., Denu, J.M., and Allis, C.D. (2001). HISTONE ACETYLTRANSFERASES. Annu. Rev. Biochem. 70, 81-120.

Saha, S., Murthy, S., and Rangarajan, P.N. (2006). Identification and characterization of a virus-inducible noncoding RNA in mouse brain. J. Gen. Virol. 87, 1991-1995.

Sakaguchi, M., and Hayashi, Y. (2012). Catching the engram: strategies to examine the memory trace. Mol Brain $5,32$. 
Schafer, D.P., Lehrman, E.K., Kautzman, A.G., Koyama, R., Mardinly, A.R., Yamasaki, R., Ransohoff, R.M., Greenberg, M.E., Barres, B.A., and Stevens, B. (2012). Microglia Sculpt Postnatal Neural Circuits in an Activity and Complement-Dependent Manner. Neuron 74, 691-705.

Schiltz, R.L., Mizzen, C.A., Vassilev, A., Cook, R.G., Allis, C.D., and Nakatani, Y. (1999). Overlapping but Distinct Patterns of Histone Acetylation by the Human Coactivators p300 and PCAF within Nucleosomal Substrates. Journal of Biological Chemistry 274, $1189-1192$.

Schor, I.E., Rascovan, N., Pelisch, F., Alló, M., and Kornblihtt, A.R. (2009). Neuronal cell depolarization induces intragenic chromatin modifications affecting NCAM alternative splicing. Proc Natl Acad Sci U S A 106, 43254330.

Schrödinger, E. (1992). What is life?: the physical aspect of the living cell ; with, Mind and matter ; \& Autobiographical sketches (Cambridge ; New York: Cambridge University Press).

Schuman, E.M., Dynes, J.L., and Steward, O. (2006). Synaptic Regulation of Translation of Dendritic mRNAs. J. Neurosci. 26, 7143-7146.

Schütz, A. (2008). Neuroanatomy. Scholarpedia 3, 3158.

Schwartz, J.H., Castellucci, V.F., and Kandel, E.R. (1971). Functioning of identified neurons and synapses in abdominal ganglion of Aplysia in absence of protein synthesis. J. Neurophysiol. 34, 939-953.

Scoville, W.B., and Millner, B. (1957). Loss of recent memory after bilateral hippocampal lesions. J. Neurol. Neurosurg. Psychiatr. 20,11-21.

Seifert, G. (2010). Langzeitpotenzierung in der CA1-Region - verschiedene Modulatoren. Text.Thesis.Doctoral. Albert-Ludwigs-Universität Freibug.

Semon, R.W. (1921). The mneme (G. Allen \& Unwin ltd.).

Shen, Y., Fu, W.-Y., Cheng, E.Y.L., Fu, A.K.Y., and Ip, N.Y. (2013). Melanocortin-4 receptor regulates hippocampal synaptic plasticity through a protein kinase A-dependent mechanism. J. Neurosci. 33, 464-472.

Shimazu, T., Hirschey, M.D., Newman, J., He, W., Shirakawa, K., Le Moan, N., Grueter, C.A., Lim, H., Saunders, L.R., Stevens, R.D., et al. (2013). Suppression of oxidative stress by $\beta$-hydroxybutyrate, an endogenous histone deacetylase inhibitor. Science 339, 211-214.

Shiotsuki, H., Yoshimi, K., Shimo, Y., Funayama, M., Takamatsu, Y., Ikeda, K., Takahashi, R., Kitazawa, S., and Hattori, N. (2010). A rotarod test for evaluation of motor skill learning. J. Neurosci. Methods 189, 180-185.

Shires, K.L., Da Silva, B.M., Hawthorne, J.P., Morris, R.G.M., and Martin, S.J. (2012). Synaptic tagging and capture in the living rat. Nat Commun 3, 1246.

Sidak, Z. (1967). Rectangular Confidence Regions for the Means of Multivariate Normal Distributions. Journal of the American Statistical Association 62, 626-633.

Da Silva, W.C., Bonini, J.S., Bevilaqua, L.R.M., Medina, J.H., Izquierdo, I., and Cammarota, M. (2008). Inhibition of mRNA synthesis in the hippocampus impairs consolidation and reconsolidation of spatial memory. Hippocampus 18, 29-39.

Sîrbu, A., Kerr, G., Crane, M., and Ruskin, H.J. (2012). RNA-Seq vs Dual- and Single-Channel Microarray Data: Sensitivity Analysis for Differential Expression and Clustering. PLoS ONE 7, e50986.

Smyth, G.K. (2004). Linear models and empirical bayes methods for assessing differential expression in microarray experiments. Stat Appl Genet Mol Biol 3, Article3.

Smyth, G.K., Michaud, J., and Scott, H.S. (2005). Use of within-array replicate spots for assessing differential expression in microarray experiments. Bioinformatics 21, 2067-2075.

Somel, M., Khaitovich, P., Bahn, S., Pääbo, S., and Lachmann, M. (2006). Gene expression becomes heterogeneous with age. Curr. Biol. 16, R359-360.

Southworth, L.K., Owen, A.B., and Kim, S.K. (2009). Aging Mice Show a Decreasing Correlation of Gene Expression within Genetic Modules. PLoS Genet 5, e1000776. 
Spedale, G., Timmers, H.T.M., and Pijnappel, W.W.M.P. (2012). ATAC-king the complexity of SAGA during evolution. Genes Dev. 26, 527-541.

Spiegel, S., Milstien, S., and Grant, S. (2012). Endogenous Modulators and Pharmacological Inhibitors of Histone Deacetylases in Cancer Therapy. Oncogene 31, 537-551.

Stearns, F.W. (2010). One Hundred Years of Pleiotropy: A Retrospective. Genetics 186, 767-773.

Stefanko, D.P., Barrett, R.M., Ly, A.R., Reolon, G.K., and Wood, M.A. (2009). Modulation of long-term memory for object recognition via HDAC inhibition. Proc. Natl. Acad. Sci. U.S.A 106, 9447-9452.

Stilling, R.M., and Fischer, A. (2011). The role of histone acetylation in age-associated memory impairment and Alzheimer's disease. Neurobiol Learn Mem 96, 19-26.

Stowell, J.C., Huot, R.I., and Van Voast, L. (1995). The synthesis of N-hydroxy-N'-phenyloctanediamide and its inhibitory effect on proliferation of AXC rat prostate cancer cells. J. Med. Chem. 38, 1411-1413.

Student (1908). The Probable Error of a Mean. Biometrika 6, 1-25.

Swank, M.W., and Sweatt, J.D. (2001). Increased histone acetyltransferase and lysine acetyltransferase activity and biphasic activation of the ERK/RSK cascade in insular cortex during novel taste learning. J. Neurosci 21, 3383-3391.

Sweatt, J.D. (2001). The neuronal MAP kinase cascade: a biochemical signal integration system subserving synaptic plasticity and memory. J. Neurochem. 76, 1-10.

Taimen, P., Pfleghaar, K., Shimi, T., Möller, D., Ben-Harush, K., Erdos, M.R., Adam, S.A., Herrmann, H., Medalia, O., Collins, F.S., et al. (2009). A progeria mutation reveals functions for lamin A in nuclear assembly, architecture, and chromosome organization. Proc. Natl. Acad. Sci. U.S.A. 106, 20788-20793.

Thangaraju, M., Gopal, E., Martin, P.M., Ananth, S., Smith, S.B., Prasad, P.D., Sterneck, E., and Ganapathy, V. (2006). SLC5A8 triggers tumor cell apoptosis through pyruvate-dependent inhibition of histone deacetylases. Cancer Res. 66, 11560-11564.

Thomas, J.O. (2001). HMG1 and 2: architectural DNA-binding proteins. Biochem. Soc. Trans. 29, 395-401.

Tollervey, J.R., Wang, Z., Hortobágyi, T., Witten, J.T., Zarnack, K., Kayikci, M., Clark, T.A., Schweitzer, A.C., Rot, G., Curk, T., et al. (2011). Analysis of alternative splicing associated with aging and neurodegeneration in the human brain. Genome Res.

Trapnell, C., Pachter, L., and Salzberg, S.L. (2009). TopHat: discovering splice junctions with RNA-Seq. Bioinformatics 25, 1105-1111.

Tsien, J.Z., Huerta, P.T., and Tonegawa, S. (1996). The essential role of hippocampal CA1 NMDA receptordependent synaptic plasticity in spatial memory. Cell $87,1327-1338$.

Tulving, E., and Donaldson, W. (1972). Organization of Memory (Academic Pr).

Tyagi, A., Ryme, J., Brodin, D., Ostlund Farrants, A.K., and Visa, N. (2009). SWI/SNF associates with nascent premRNPs and regulates alternative pre-mRNA processing. PLoS Genet 5, e1000470.

Ullman, M.T. (2001). A neurocognitive perspective on language: the declarative/procedural model. Nat. Rev. Neurosci. 2, 717-726.

Valor, L.M., Pulopulos, M.M., Jimenez-Minchan, M., Olivares, R., Lutz, B., and Barco, A. (2011). Ablation of CBP in Forebrain Principal Neurons Causes Modest Memory and Transcriptional Defects and a Dramatic Reduction of Histone Acetylation But Does Not Affect Cell Viability. J. Neurosci 31, 1652-1663.

Varisli, L. (2012). Meta-analysis of the expression of the mitosis-related gene Fam83D. Oncol Lett 4, 1335-1340.

Värv, S., Kristjuhan, K., Peil, K., Lõoke, M., Mahlakõiv, T., Paapsi, K., and Kristjuhan, A. (2010). Acetylation of H3 K56 is required for RNA polymerase II transcript elongation through heterochromatin in yeast. Mol. Cell. Biol $30,1467-1477$. 
Vazdarjanova, A., Ramirez-Amaya, V., Insel, N., Plummer, T.K., Rosi, S., Chowdhury, S., Mikhael, D., Worley, P.F., Guzowski, J.F., and Barnes, C.A. (2006). Spatial exploration induces ARC, a plasticity-related immediate-early gene, only in calcium/calmodulin-dependent protein kinase II-positive principal excitatory and inhibitory neurons of the rat forebrain. J. Comp. Neurol. 498, 317-329.

Vecsey, C.G., Hawk, J.D., Lattal, K.M., Stein, J.M., Fabian, S.A., Attner, M.A., Cabrera, S.M., McDonough, C.B., Brindle, P.K., Abel, T., et al. (2007). Histone Deacetylase Inhibitors Enhance Memory and Synaptic Plasticity via CREB: CBP-Dependent Transcriptional Activation. J. Neurosci. 27, 6128-6140.

Verbitsky, M., Yonan, A.L., Malleret, G., Kandel, E.R., Gilliam, T.C., and Pavlidis, P. (2004). Altered Hippocampal Transcript Profile Accompanies an Age-Related Spatial Memory Deficit in Mice. Learning \& Memory 11, 253260.

Vernarecci, S., Tosi, F., and Filetici, P. (2010). Tuning acetylated chromatin with HAT inhibitors: a novel tool for therapy. Epigenetics 5, 105-111.

Vogel-Ciernia, A., and Wood, M.A. (2012). Molecular brake pad hypothesis: pulling off the brakes for emotional memory. Reviews in the Neurosciences 1-20.

Waddington, C.H. (1953). Epigenetics and Evolution. Symp. Soc. Exp. Biol. 7, 186-199.

Walker, L.C., and LeVine, H. (2000). The cerebral proteopathies: neurodegenerative disorders of protein conformation and assembly. Mol. Neurobiol. 21, 83-95.

Welle, S., Brooks, A.I., Delehanty, J.M., Needler, N., and Thornton, C.A. (2003). Gene expression profile of aging in human muscle. Physiol. Genomics 14, 149-159.

West, M.J. (1990). Stereological studies of the hippocampus: a comparison of the hippocampal subdivisions of diverse species including hedgehogs, laboratory rodents, wild mice and men. Prog. Brain Res. 83, 13-36.

Winston, F., and Allis, C.D. (1999). The bromodomain: a chromatin-targeting module? Nat Struct Mol Biol 6, 601604.

Wood, M.A., Kaplan, M.P., Park, A., Blanchard, E.J., Oliveira, A.M.M., Lombardi, T.L., and Abel, T. (2005). Transgenic mice expressing a truncated form of CREB-binding protein (CBP) exhibit deficits in hippocampal synaptic plasticity and memory storage. Learn. Mem. 12, 111-119.

Wood, M.A., Attner, M.A., Oliveira, A.M.M., Brindle, P.K., and Abel, T. (2006). A transcription factor-binding domain of the coactivator CBP is essential for long-term memory and the expression of specific target genes. Learn. Mem. 13, 609-617.

Woollett, K., and Maguire, E.A. (2011). Acquiring "the Knowledge" of London's layout drives structural brain changes. Curr. Biol. 21, 2109-2114.

Wyss-Coray, T., and Rogers, J. (2012). Inflammation in Alzheimer Disease-A Brief Review of the Basic Science and Clinical Literature. Cold Spring Harb Perspect Med 2.

Wyss-Coray, T., Yan, F., Lin, A.H.-T., Lambris, J.D., Alexander, J.J., Quigg, R.J., and Masliah, E. (2002). Prominent neurodegeneration and increased plaque formation in complement-inhibited Alzheimer's mice. Proc. Natl. Acad. Sci. U.S.A. 99, 10837-10842.

Xu, X., Zhan, M., Duan, W., Prabhu, V., Brenneman, R., Wood, W., Firman, J., Li, H., Zhang, P., Ibe, C., et al. (2007). Gene expression atlas of the mouse central nervous system: impact and interactions of age, energy intake and gender. Genome Biol. 8, R234.

Yankner, B.A., Lu, T., and Loerch, P. (2008). The Aging Brain. Annu. Rev. Pathol. Mech. Dis. 3, 41-66.

Zahn, J.M., Poosala, S., Owen, A.B., Ingram, D.K., Lustig, A., Carter, A., Weeraratna, A.T., Taub, D.D., Gorospe, M., Mazan-Mamczarz, K., et al. (2007). AGEMAP: A Gene Expression Database for Aging in Mice. PLos Genet 3, e201.

Zeng, Q., Subramaniam, V.N., Wong, S.H., Tang, B.L., Parton, R.G., Rea, S., James, D.E., and Hong, W. (1998). A Novel Synaptobrevin/VAMP Homologous Protein (VAMP5) Is Increased during In Vitro Myogenesis and Present in the Plasma Membrane. Mol. Biol. Cell 9, 2423-2437.

Zeng, Q., Tran, T.T.H., Tan, H.-X., and Hong, W. (2003). The Cytoplasmic Domain of Vamp4 and Vamp5 Is Responsible for Their Correct Subcellular Targeting THE N-TERMINAL EXTENSION OF VAMP4 CONTAINS A 
DOMINANT AUTONOMOUS TARGETING SIGNAL FOR THE TRANS-GOLGI NETWORK. J. Biol. Chem. 278, 2304623054.

Zeng, Y., Tan, M., Kohyama, J., Sneddon, M., Watson, J.B., Sun, Y.E., and Xie, C.-W. (2011). Epigenetic Enhancement of BDNF Signaling Rescues Synaptic Plasticity in Aging. The Journal of Neuroscience 31, $17800-$ 17810.

Zhou, B.O., Wang, S.-S., Zhang, Y., Fu, X.-H., Dang, W., Lenzmeier, B.A., and Zhou, J.-Q. (2011a). Histone H4 Lysine 12 Acetylation Regulates Telomeric Heterochromatin Plasticity in Saccharomyces cerevisiae. PLoS Genet 7, e1001272.

Zhou, H.-L., Hinman, M.N., Barron, V.A., Geng, C., Zhou, G., Luo, G., Siegel, R.E., and Lou, H. (2011b). Hu proteins regulate alternative splicing by inducing localized histone hyperacetylation in an RNA-dependent manner. Proc Natl Acad Sci U S A.

Babraham Bioinformatics - FastQC A Quality Control tool for High Throughput Sequence Data.

HTSeq: Analysing high-throughput sequencing data with Python.

Babraham Bioinformatics - SeqMonk Mapped Sequence Analysis Tool. 


\section{Appendix}

\subsection{Supplementary Figures}

6.1.1. Expression pattern of Kat2a in the adult murine brain from the Allen Brain Atlas

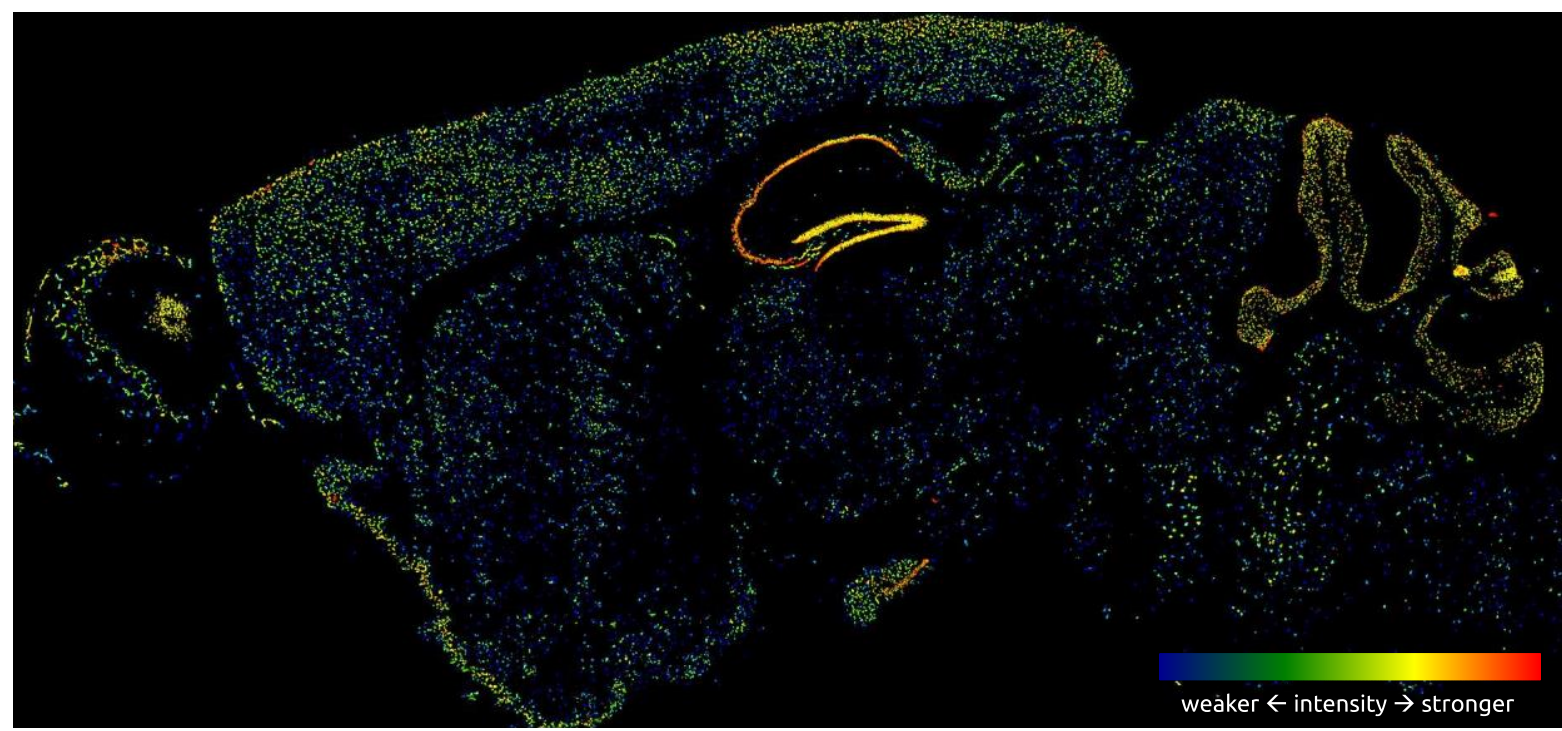

Figure 6-1: IN SITU hybridization image of the adult murine brain, sagittal section. Image adapted from the Allen Brain Atlas, mouse.brainmap.org

6.1.2. Robust AAV-mediated expression of MYC-tagged Kat2a 14 days after injection

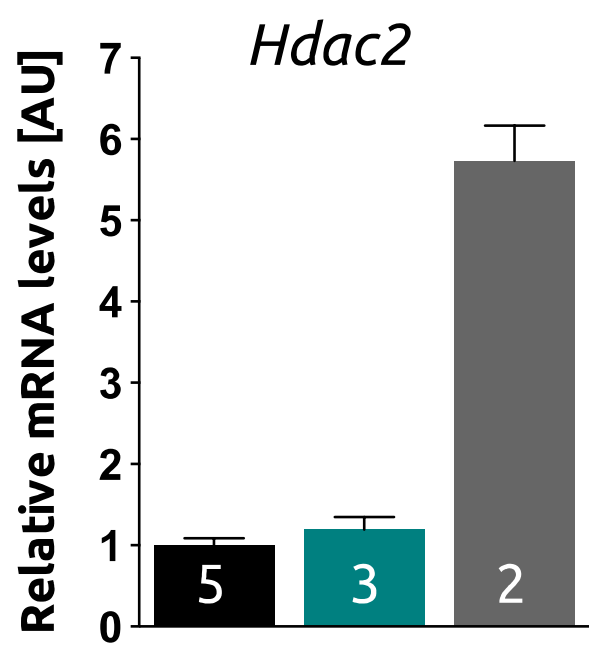

AAV

Figure 6-2: Hdac2 mRNA levels 14 days after injection. mRNA levels of Hdac2 were almost 6-fold increased in mice injected with Hdac2-MYC-AAV compared to mice injected with either GFP-AAV or Kat2a-MYC-AAV. 


\subsubsection{Strong Accumulation of Autofluorescent Lipofuscin in Hippocampal Microglia}

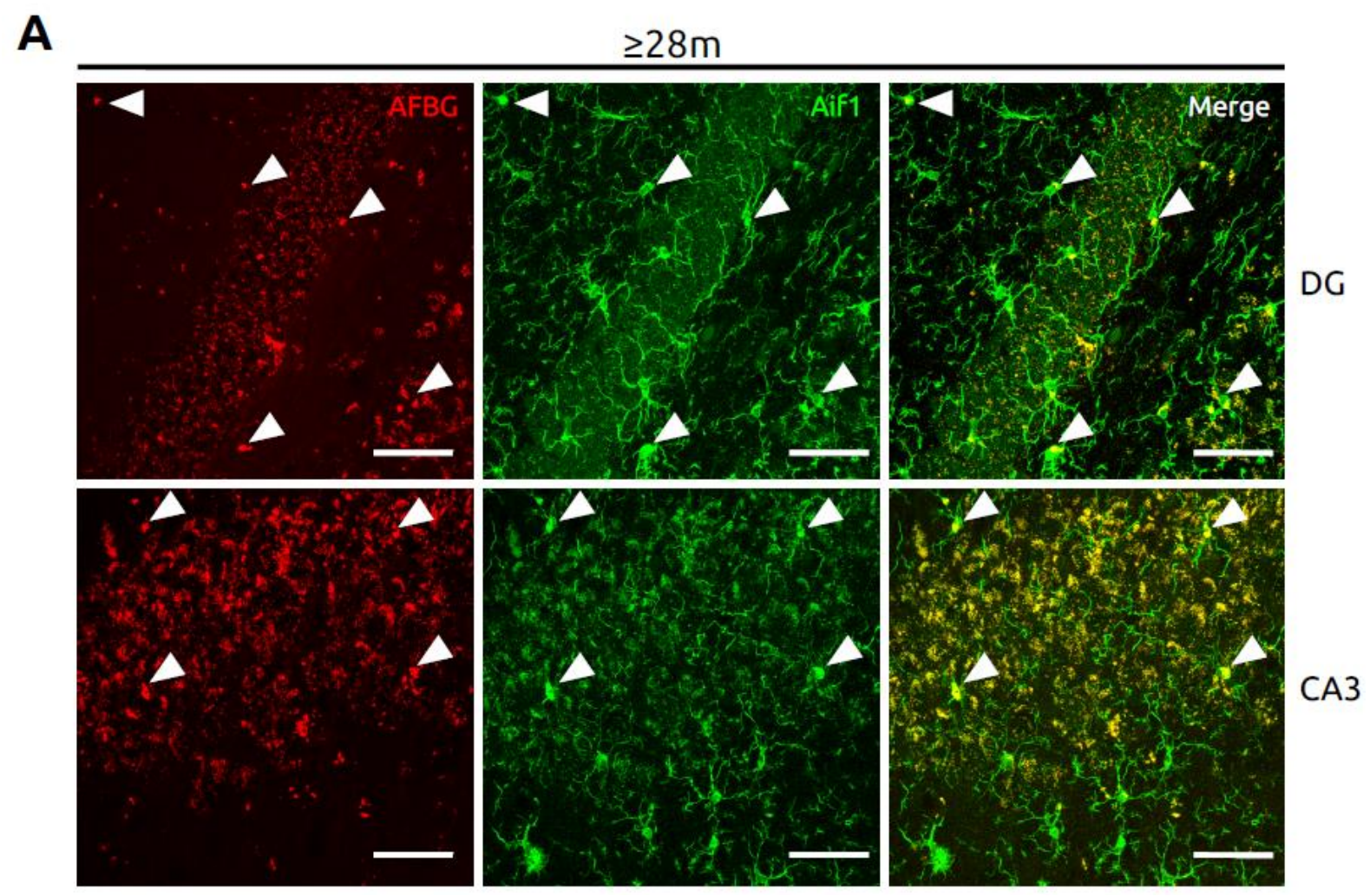

Figure 6-3: Lipofuscin in DG and CA3 co-stained with microglia. CA3 (lower panels) pyramidal neurons seem to contain bigger and brighter aggregates compared to DG (upper panels) granule cells in $\geq 28$-month-old mice. Some of the brightest autofluorescent spots co-localize with $\mathrm{Iba1} 1^{+}$(Aif1 ${ }^{+}$) activated microglia (white arrowheads, A). Scale bar is $50 \mu \mathrm{m}$. 


\subsubsection{Overview of the complement system and coagulation pathyway}

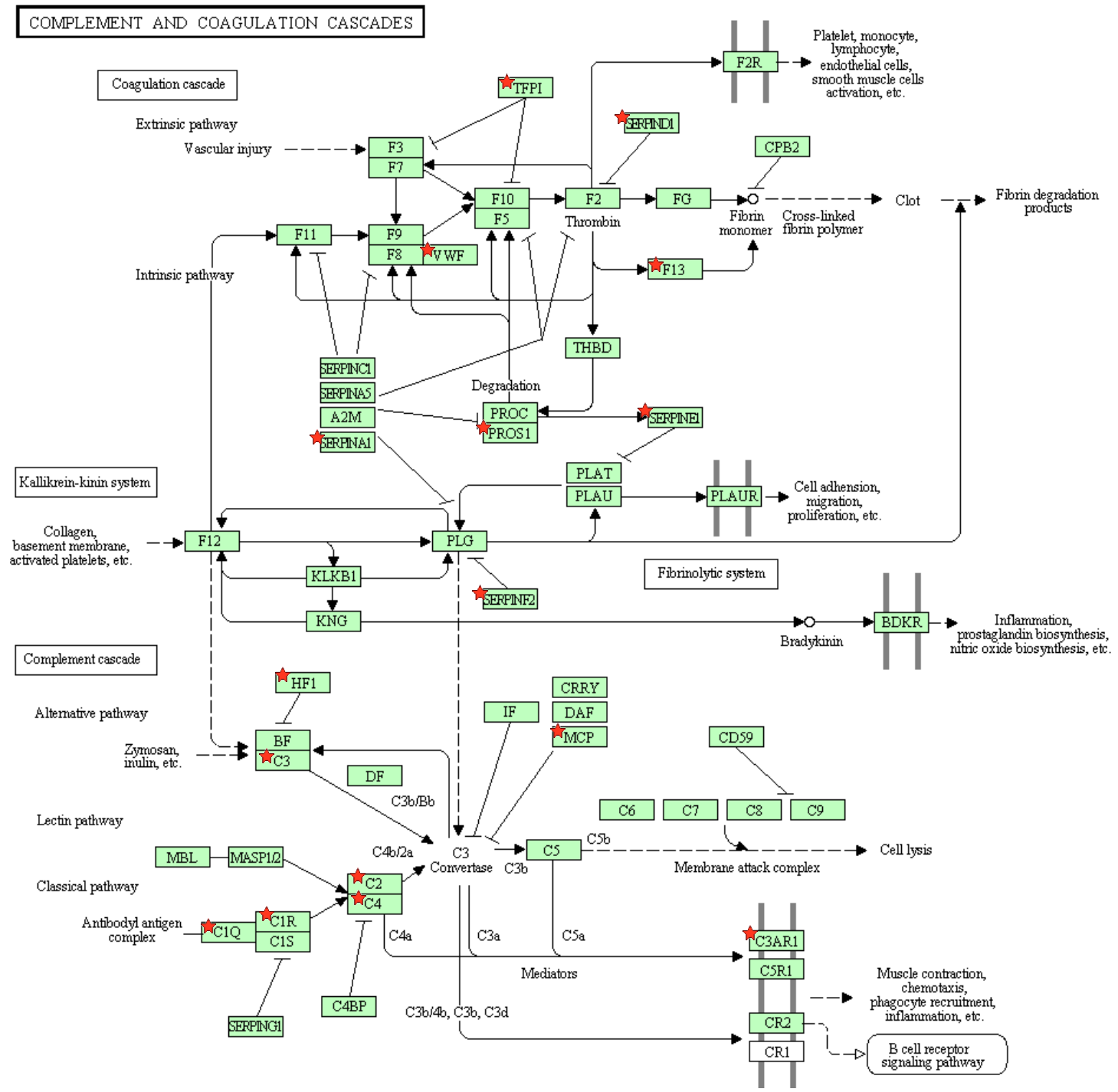

Figure 6-4: KEGG pathway for complement and coagulation cascades. Red stars mark the genes found to be differentially regulated in the study of transcriptional changes during hippocampal aging. 


\subsection{Supplementary Tables}

\subsubsection{List of DEGs in the study of Kat2a function}

The following table contains gene names called as DEGs in pairwise comparisons during the study of Kat2a function in stimulus-dependent gene expression. DEGs were defined as genes with a $\log _{2}=0.5$ - fold change at a significance level of $p_{a d j}<0.1$. The lists are sorted by descending fold-change.

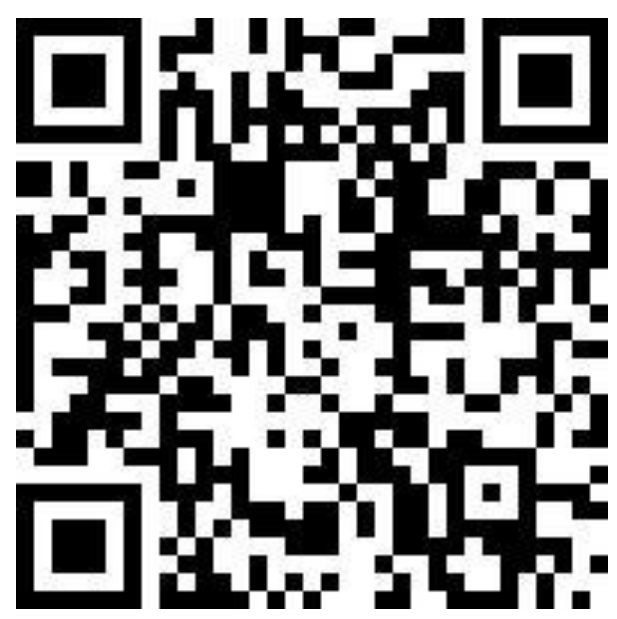

QR 1: https://dl.dropbox.com/u/1715727/Supplementary_Table_6.2.1.zip

Password protected zip-file (70 kilobyte)

PW: stl921 


\subsubsection{Lists of significantly overrepresented GO-Terms and KEGG-Pathways in the study of Kat2a function}

Table 6-1: Comparison of group A and group B. For "Merged " a merged list of up- and downregulated genes was used as input for functional annotation.

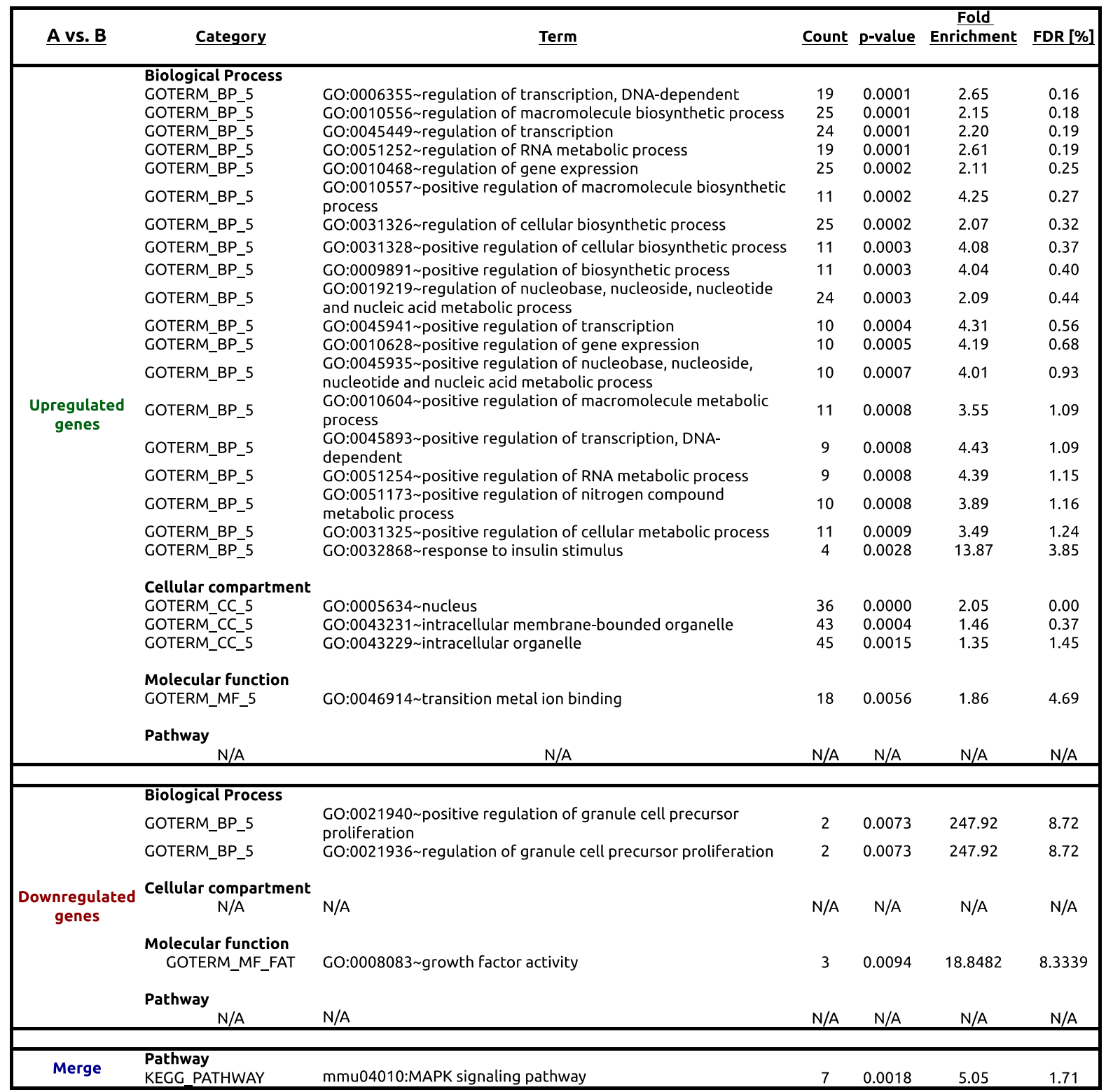


Table 6-2: Comparison of group A and group C. For "Merged " a merged list of up- and downregulated genes was used as input for functional annotation.

\begin{tabular}{|c|c|c|c|c|c|c|}
\hline A vs. C & Category & $\underline{T e r m}$ & Count & p-value & $\begin{array}{c}\text { Fold } \\
\text { Enrichment }\end{array}$ & FDR [\%] \\
\hline \multirow{5}{*}{$\begin{array}{l}\text { Upregulated } \\
\text { genes }\end{array}$} & $\begin{array}{c}\text { Biological Process } \\
\text { N/A }\end{array}$ & \multirow[b]{2}{*}{ GO:0005886 plasma membrane } & N/A & N/A & N/A & N/A \\
\hline & $\begin{array}{l}\text { Cellular compartment } \\
\text { GOTERM_CC_4 }\end{array}$ & & 17 & 0.0054 & 2.0 & 5.76 \\
\hline & $\begin{array}{c}\text { Molecular function } \\
\text { N/A }\end{array}$ & $\mathrm{N} / \mathrm{A}$ & N/A & N/A & N/A & N/A \\
\hline & Pathway & & & & & \\
\hline & $\mathrm{N} / \mathrm{A}$ & $\mathrm{N} / \mathrm{A}$ & N/A & $\mathrm{N} / \mathrm{A}$ & $\mathrm{N} / \mathrm{A}$ & $\mathrm{N} / \mathrm{A}$ \\
\hline \multirow{15}{*}{$\begin{array}{l}\text { Downregulated } \\
\text { genes }\end{array}$} & Biological Process & \multirow[b]{2}{*}{$\begin{array}{l}\text { GO:0007200 activation of phospholipase C activity by G- } \\
\text { protein coupled receptor protein signaling pathway coupled } \\
\text { to IP3 second messenger }\end{array}$} & & & & \\
\hline & GOTERM_BP_FAT & & 3 & 0.0008 & 67.9 & 1.07714 \\
\hline & GOTERM_BP_FAT & GO:0010863 positive regulation of phospholipase C activity & 3 & 0.0009 & 65.3 & 1.16522 \\
\hline & GOTERM_BP_FAT & \multirow{7}{*}{$\begin{array}{l}\text { GO:0010518 positive regulation of phospholipase activity } \\
\text { GO:0007202 activation of phospholipase C activity } \\
\text { GO:0060193 positive regulation of lipase activity } \\
\text { GO:0010517 regulation of phospholipase activity } \\
\text { GO:0060191 regulation of lipase activity } \\
\text { GO:0048015 phosphoinositide-mediated signaling } \\
\text { GO:0007208 activation of phospholipase C activity by } \\
\text { serotonin receptor signaling pathway }\end{array}$} & 3 & 0.0009 & 65.3 & 1.16522 \\
\hline & GOTERM_BP_FAT & & 3 & 0.0009 & 65.3 & 1.16522 \\
\hline & GOTERM_BP_FAT & & 3 & 0.0009 & 62.9 & 1.25661 \\
\hline & GOTERM_BP_FAT & & 3 & 0.001 & 60.7 & 1.35128 \\
\hline & GOTERM_BP_FAT & & 3 & 0.0013 & 53.1 & 1.76242 \\
\hline & GOTERM_BP_FAT & & 3 & 0.0021 & 42.5 & 2.73625 \\
\hline & GOTERM_BP_FAT & & 2 & 0.0051 & 377.4 & 6.62283 \\
\hline & \multicolumn{2}{|l|}{ Cellular compartment } & \multirow[b]{2}{*}{ N/A } & \multirow[b]{2}{*}{ N/A } & \multirow[b]{2}{*}{ N/A } & \multirow[b]{2}{*}{ N/A } \\
\hline & N/A & $\mathrm{N} / \mathrm{A}$ & & & & \\
\hline & $\begin{array}{l}\text { Molecular function } \\
\text { N/A }\end{array}$ & N/A & N/A & N/A & N/A & N/A \\
\hline & \multirow{2}{*}{$\begin{array}{l}\text { Pathway } \\
\text { KEGG PATHWAY }\end{array}$} & \multirow[b]{2}{*}{ mmu04080:Neuroactive ligand-receptor interaction } & \multirow[b]{2}{*}{4} & \multirow[b]{2}{*}{0.0063} & \multirow[b]{2}{*}{8.8} & \multirow[b]{2}{*}{9.26} \\
\hline & & & & & & \\
\hline Merge & $\begin{array}{l}\text { Pathway } \\
\text { KEGG_PATHWAY }\end{array}$ & mmu04080:Neuroactive ligand-receptor interaction & 6 & 0.0040 & 5.3 & 3.50 \\
\hline
\end{tabular}


Table 6-3: Comparison of group B and group D. For "Merged " a merged list of up- and downregulated genes was used as input for functional annotation.

\begin{tabular}{|c|c|c|c|c|c|c|}
\hline B vs. D & Category & Term & Count & p-value & $\begin{array}{c}\text { Fold } \\
\text { Enrichment }\end{array}$ & FDR [\%] \\
\hline $\begin{array}{l}\text { Upregulated } \\
\text { genes }\end{array}$ & $\begin{array}{l}\text { Biological Process } \\
\text { GOTERM_BP_5 } \\
\text { Cellular compartment } \\
\text { N/A } \\
\text { Molecular function } \\
\text { GOTERM_MF_5 } \\
\text { KEGG-Pathway } \\
\text { KEGG_PATHWAY } \\
\text { KEGG_PATHWAY }\end{array}$ & $\begin{array}{l}\text { GO:0007283 spermatogenesis } \\
\text { N/A } \\
\text { GO:0005509 calcium ion binding } \\
\text { mmu04512:ECM-receptor interaction } \\
\text { mmu04510:Focal adhesion }\end{array}$ & $\begin{array}{l}7 \\
\text { N/A } \\
13 \\
\\
5 \\
6\end{array}$ & $\begin{array}{l}0.0060 \\
\text { N/A } \\
0.0047 \\
0.0006 \\
0.0024\end{array}$ & $\begin{array}{l}4.21 \\
\text { N/A } \\
2.47 \\
11.92 \\
6.00\end{array}$ & $\begin{array}{l}7.83 \\
\text { N/A } \\
4.56 \\
\\
0.62 \\
2.30 \\
\end{array}$ \\
\hline $\begin{array}{c}\text { Downregulated } \\
\text { genes }\end{array}$ & $\begin{array}{l}\text { Biological Process } \\
\text { PANTHER_BP_ALL } \\
\text { Cellular compartment } \\
\text { GOTERM_CC_5 } \\
\text { Molecular function } \\
\text { GOTERM_MF_FAT } \\
\text { GOTERM_MF_FAT } \\
\text { KEGG-Pathway } \\
\text { KEGG_PATHWAY } \\
\end{array}$ & $\begin{array}{l}\text { BP00166: Neuronal activities } \\
\text { GO:0005840 ribosome } \\
\text { GO:0004993 serotonin receptor activity } \\
\text { GO:0003735 structural constituent of ribosome } \\
\text { mmu03010:Ribosome }\end{array}$ & $\begin{array}{l}3 \\
5\end{array}$ & $\begin{array}{l}0.0043 \\
0.0022 \\
0.0008 \\
0.0011 \\
0.0005 \\
\end{array}$ & $\begin{array}{l}3.76 \\
8.83 \\
69.45 \\
10.73 \\
12.90 \\
\end{array}$ & $\begin{array}{l}2.17 \\
0.90 \\
1.22 \\
0.39 \\
\end{array}$ \\
\hline Merge & $\begin{array}{l}\text { KEGG-Pathway } \\
\text { KEGG_PATHWAY } \\
\text { KEGG_PATHWAY } \\
\text { KEGG_PATHWAY }\end{array}$ & $\begin{array}{l}\text { mmu04080:Neuroactive ligand-receptor interaction } \\
\text { mmu04512:ECM-receptor interaction } \\
\text { mmu03010:Ribosome }\end{array}$ & $\begin{array}{l}10 \\
6 \\
5\end{array}$ & $\begin{array}{l}0.0005 \\
0.0009 \\
0.0089\end{array}$ & $\begin{array}{l}4.09 \\
7.68 \\
5.97\end{array}$ & $\begin{array}{l}0.56 \\
0.97 \\
8.91\end{array}$ \\
\hline
\end{tabular}


Table 6-4: Comparison of group C and group D. For "Merged " a merged list of up- and downregulated genes was used as input for functional annotation.

\begin{tabular}{|c|c|c|c|c|c|c|}
\hline C vs. D & Category & $\underline{\text { Term }}$ & Count & p-value & $\begin{array}{c}\text { Fold } \\
\text { Enrichment }\end{array}$ & FDR [\%] \\
\hline \multirow{15}{*}{$\begin{array}{l}\text { Upregulated } \\
\text { genes }\end{array}$} & Biological Process & & & & & \\
\hline & GOTERM_BP_5 & \multirow{2}{*}{$\begin{array}{l}\text { GO:0019219 regulation of nucleobase, nucleoside, nucleotide } \\
\text { and nucleic acid metabolic process } \\
\text { GO:0045449 regulation of transcription }\end{array}$} & 25 & 0.0006 & 1.98 & 0.81 \\
\hline & GOTERM_BP_5 & & 24 & 0.0007 & 2.00 & 0.93 \\
\hline & GOTERM_BP_5 & & 25 & 0.0012 & 1.88 & 1.63 \\
\hline & GOTERM_BP_5 & GO:0006355 regulation of transcription, DNA-dependent & 18 & 0.0013 & 2.28 & 1.68 \\
\hline & GOTERM_BP_5 & & 18 & 0.0015 & 2.25 & 1.99 \\
\hline & GOTERM_BP_5 & GO:0010556 regulation of macromolecule biosynthetic process & 24 & 0.0018 & 1.87 & 2.39 \\
\hline & GOTERM_BP_5 & GO:0010468 regulation of gene expression & 24 & 0.0024 & 1.84 & 3.12 \\
\hline & Cellular compartment & & & & & \\
\hline & GOTERM_CC_5 & & 37 & 0.0000 & 1.88 & 0.01 \\
\hline & GOTERM_CC_5 & GO:0043231 intracellular membrane-bounded organelle & 48 & 0.0002 & 1.45 & 0.20 \\
\hline & GOTERM_CC_5 & GO:0043229 intracellular organelle & 49 & 0.0027 & 1.31 & 2.68 \\
\hline & $\begin{array}{l}\text { Molecular function } \\
\text { GOTERM_MF_5 }\end{array}$ & GO:0046914 transition metal ion binding & 22 & 0.0045 & 1.76 & 4.02 \\
\hline & KEGG-Pathway & \multirow[b]{2}{*}{ mmu04010:MAPK signaling pathway } & & & & \\
\hline & KEGG_PATHWAY & & 7 & 0.0005 & 6.32 & 0.43 \\
\hline \multirow{8}{*}{$\begin{array}{l}\text { Downregulated } \\
\text { genes }\end{array}$} & \multicolumn{2}{|l|}{ Biological Process } & & & & \\
\hline & N/A & N/A & N/A & N/A & N/A & N/A \\
\hline & \multicolumn{2}{|l|}{ Cellular compartment } & & & & \\
\hline & N/A & N/A & N/A & N/A & N/A & N/A \\
\hline & \multicolumn{2}{|l|}{ Molecular function } & & & & \\
\hline & N/A & N/A & N/A & N/A & N/A & N/A \\
\hline & \multicolumn{2}{|l|}{ KEGG-Pathway } & & & & \\
\hline & $\mathrm{N} / \mathrm{A}$ & N/A & N/A & N/A & N/A & $\mathrm{N} / \mathrm{A}$ \\
\hline Merge & $\begin{array}{l}\text { KEGG-Pathway } \\
\text { see upregulated }\end{array}$ & & & & & \\
\hline
\end{tabular}


6.2.3. List of DEGs in the study of transcriptional changes during hippocampal aging

The following table contains gene names called as DEGs in pairwise comparisons during the study of transcriptional changes during hippocampal aging. DEGs were defined as genes with a $\log _{2}=0.5-$ fold change at a significance level of $\mathrm{p}_{\mathrm{adj}}<0.1$. The lists are sorted by descending fold-change.

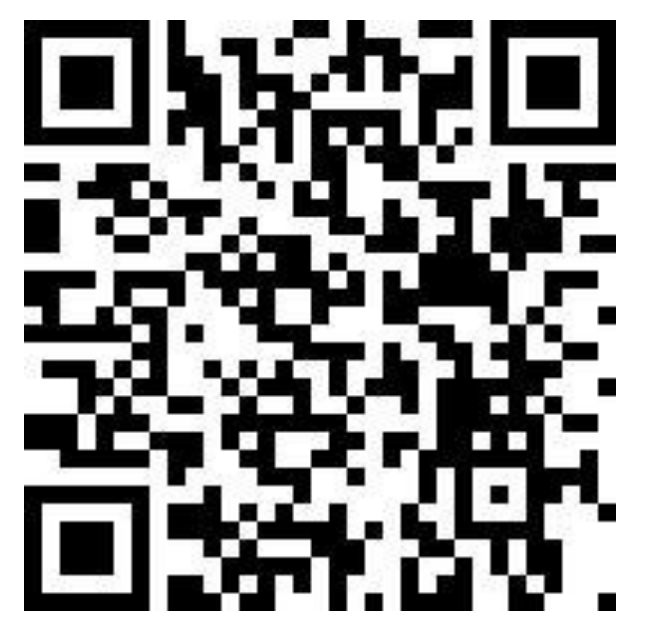

QR 2: https://dl.dropbox.com/u/1715727/Supplementary_Table_6.2.3.zip

Password protected zip-file (82 kilobyte)

PW: stl923 


\subsubsection{Lists of Significantly Overrepresented GO-Terms and KEGG-Pathways in the}

\section{Study of Hippocampal Aging}

\section{Table 6-5: Comparison of $3 \mathrm{~m}$ and $24 \mathrm{~m}$ using RNA-seq}

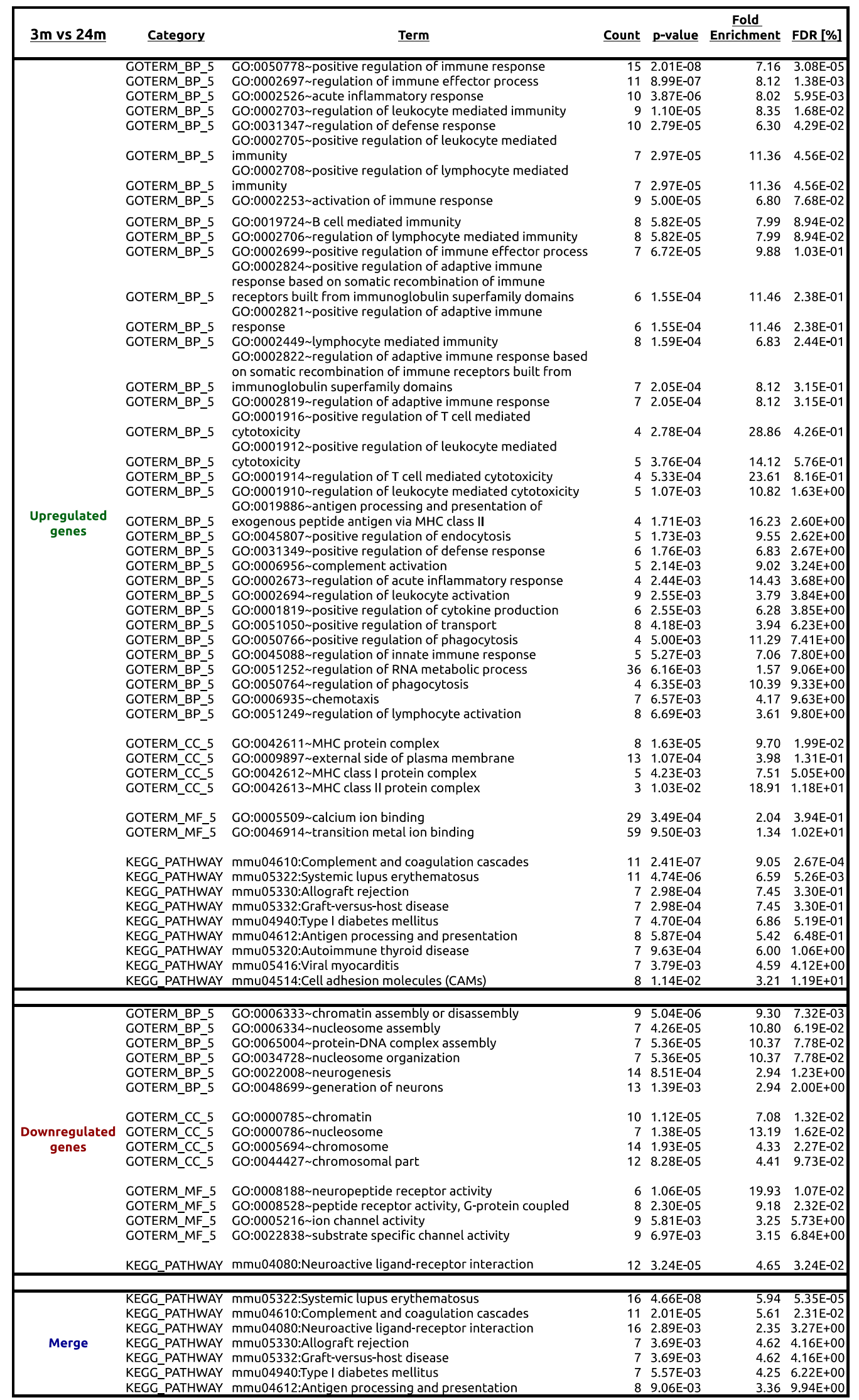


Table 6-6: Comparison of $3 \mathrm{~m}$ and $\geq 28 \mathrm{~m}$ using RNA-seq

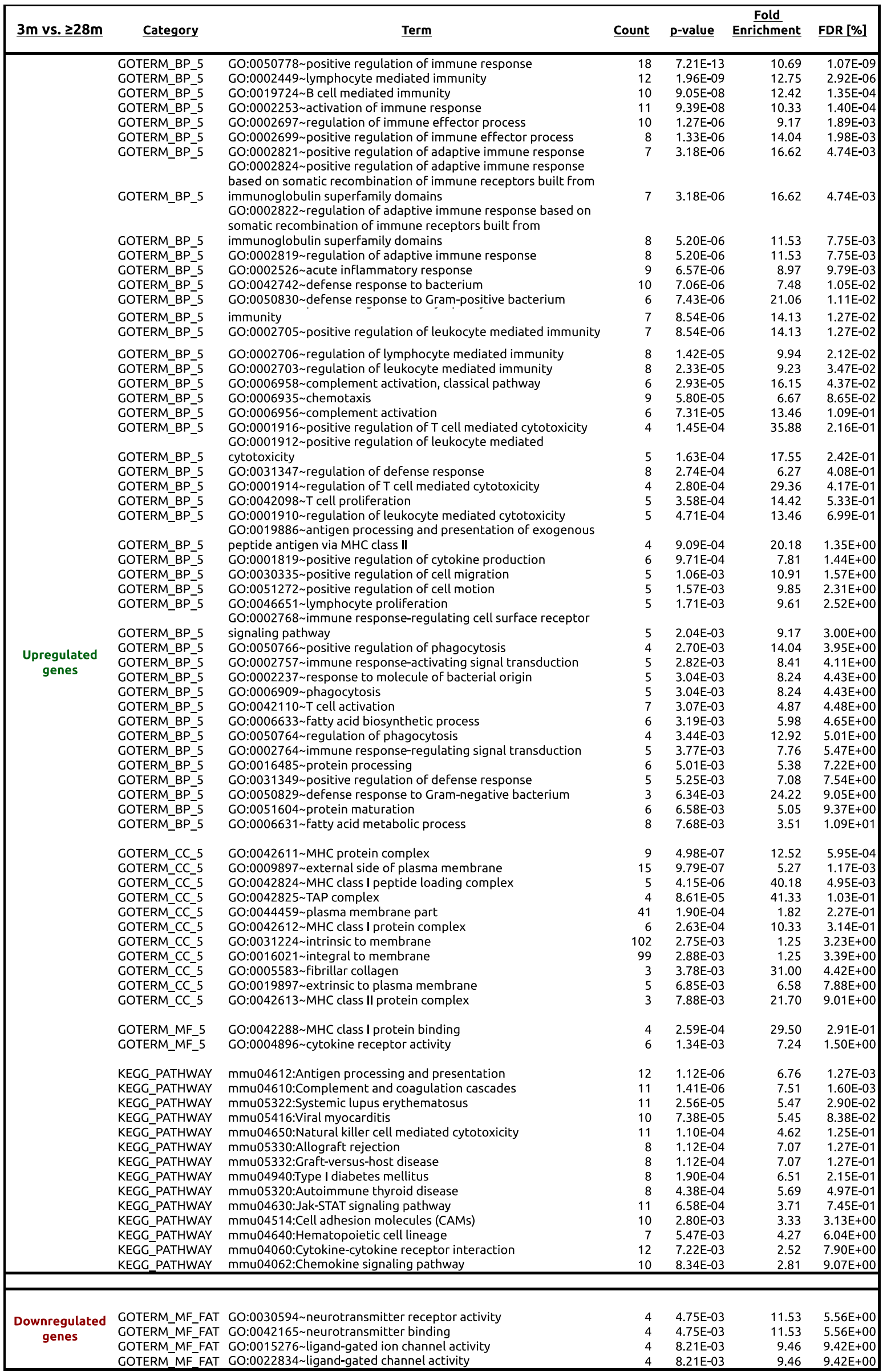


Table 6-7: Comparison of $3 \mathrm{~m}$ and $\geq 28 \mathrm{~m}$ using microarrays

\begin{tabular}{|c|c|c|c|c|c|c|}
\hline$\frac{3 \text { vs. } \geq 28 m}{\text { Array }}$ & Category & Term & Count & p-value & $\begin{array}{c}\text { Fold } \\
\text { Enrichment }\end{array}$ & FDR [\%] \\
\hline \multirow{39}{*}{$\begin{array}{l}\text { Upregulated } \\
\text { genes }\end{array}$} & $\begin{array}{l}\text { GOTERM_BP_5 } \\
\text { GOTERM_BP_5 } \\
\text { GOTERM_BP_5 } \\
\text { GOTERM_BP_5 } \\
\text { GOTERM_BP_5 } \\
\text { GOTERM_BP_5 } \\
\text { GOTERM_BP_5 } \\
\text { GOTERM_BP_5 }\end{array}$ & $\begin{array}{l}\text { GO:0002526 acute inflammatory response } \\
\text { GO:0002449 lymphocyte mediated immunity } \\
\text { GO:0019724 B cell mediated immunity } \\
\text { GO:0006935 chemotaxis } \\
\text { GO:0050778 positive regulation of immune response } \\
\text { GO:0001819 positive regulation of cytokine production } \\
\text { GO:0006958 complement activation, classical pathway } \\
\text { GO:0031347 regulation of defense response }\end{array}$ & $\begin{array}{r}12 \\
11 \\
10 \\
11 \\
12 \\
8 \\
6 \\
9\end{array}$ & $\begin{array}{l}1.54 \mathrm{E}-08 \\
9.68 \mathrm{E}-08 \\
2.76 \mathrm{E}-07 \\
2.90 \mathrm{E}-06 \\
3.19 \mathrm{E}-06 \\
2.42 \mathrm{E}-05 \\
5.47 \mathrm{E}-05 \\
9.70 \mathrm{E}-05\end{array}$ & $\begin{array}{r}10.51 \\
10.27 \\
10.91 \\
7.16 \\
6.26 \\
9.15 \\
14.19 \\
6.20\end{array}$ & \begin{tabular}{r|}
$2.40 \mathrm{E}-05$ \\
$1.51 \mathrm{E}-04$ \\
$4.29 \mathrm{E}-04$ \\
0.004519831 \\
0.00496257 \\
0.037604796 \\
0.085154703 \\
0.150926775 \\
\end{tabular} \\
\hline & $\begin{array}{l}\text { GOTERM_BP_5 } \\
\text { GOTERM_BP_5 } \\
\text { GOTERM_BP_5 } \\
\text { GOTERM_BP_5 } \\
\text { GOTERM_BP_5 } \\
\text { GOTERM_BP_5 } \\
\text { GOTERM_BP_5 } \\
\text { GOTERM_BP_5 } \\
\text { GOTERM_BP_5 }\end{array}$ & $\begin{array}{l}\text { GO:0006956 complement activation } \\
\text { GO:0002253 activation of immune response } \\
\text { GO:0002697 regulation of immune effector process } \\
\text { GO:0016485 protein processing } \\
\text { GO:0051604 protein maturation } \\
\text { GO:0002699 positive regulation of immune effector process } \\
\text { GO:0051050 positive regulation of transport } \\
\text { GO:0031349 positive regulation of defense response } \\
\text { GO:0045807 positive regulation of endocytosis } \\
\text { GO:0019886 antigen processing and presentation of exogenous }\end{array}$ & $\begin{array}{l}6 \\
8 \\
8 \\
8 \\
8 \\
6 \\
9 \\
6 \\
5\end{array}$ & $\begin{array}{r}1.35 \mathrm{E}-04 \\
1.99 \mathrm{E}-04 \\
2.30 \mathrm{E}-04 \\
2.64 \mathrm{E}-04 \\
3.93 \mathrm{E}-04 \\
4.39 \mathrm{E}-04 \\
5.33 \mathrm{E}-04 \\
0.001185759 \\
0.001244601\end{array}$ & $\begin{array}{r}11.82 \\
6.60 \\
6.45 \\
6.31 \\
5.91 \\
9.25 \\
4.84 \\
7.47 \\
10.43\end{array}$ & $\begin{array}{l}0.210350673 \\
0.309606574 \\
0.357245218 \\
0.410608423 \\
0.610064377 \\
0.681620303 \\
0.826794622 \\
1.829511564 \\
1.919479203\end{array}$ \\
\hline & $\begin{array}{l}\text { GOTERM_BP_5 } \\
\text { GOTERM_BP_5 }\end{array}$ & $\begin{array}{l}\text { peptide antigen via MHC class II } \\
\text { GO:0002706 regulation of lymphocyte mediated immunity }\end{array}$ & $\begin{array}{l}4 \\
6\end{array}$ & $\begin{array}{l}0.001324699 \\
0.002138162\end{array}$ & $\begin{array}{r}17.73 \\
6.55\end{array}$ & $\begin{array}{l}2.041820946 \\
3.276246394\end{array}$ \\
\hline & GOTERM_BP_5 & GO:0002705 positive regulation of leukocyte mediated immunity & 5 & 0.002297837 & 8.87 & 3.516836587 \\
\hline & GOTERM_BP_5 & GO:0002708 positive regulation of lymphocyte mediated immunity & 5 & 0.002297837 & 8.87 & 3.516836587 \\
\hline & GOTERM_BP_5 & GO:0043065 positive regulation of apoptosis & 11 & 0.002586379 & 3.15 & 3.950178791 \\
\hline & GOTERM_BP_5 & GO:0043068 positive regulation of programmed cell death & 11 & 0.002751407 & 3.12 & 4.197203439 \\
\hline & GOTERM_BP_5 & GO:0009967 positive regulation of signal transduction & 9 & 0.002912649 & 3.71 & 4.437985679 \\
\hline & GOTERM_BP_5 & GO:0010942 positive regulation of cell death & 11 & 0.002935499 & 3.10 & 4.472062013 \\
\hline & GOTERM_BP_5 & GO:0002703 regulation of leukocyte mediated immunity & 6 & 0.002962275 & 6.08 & 4.511979368 \\
\hline & GOTERM_BP_5 & GO:0009888 tissue development & 19 & 0.003649197 & 2.11 & 5.530692569 \\
\hline & GOTERM_BP_5 & GO:0050766 positive regulation of phagocytos & 4 & 0.003898568 & 12.34 & 5.897990017 \\
\hline & GOTERM_BP_5 & GO:0042742 defense response to bacterium & 7 & 0.004092833 & 4.60 & 6.183195013 \\
\hline & GOTERM_BP_5 & GO:0042981 regulation of apoptosis & 17 & 0.004773516 & 2.18 & 7.176154422 \\
\hline & GOTERM BP 5 & GO:0006909 phagocytosis & 5 & 0.004832484 & 7.24 & 7.261709941 \\
\hline & GOTERM_BP_5 & GO:0050764 regulation of phagocytosis & 4 & 0.004960381 & 11.35 & 7.44702094 \\
\hline & GOTERM_BP_5 & GO:0006897 endocytosis & 9 & 0.004993081 & 3.40 & 7.494344837 \\
\hline & GOTERM_BP_5 & GO:0010647 positive regulation of cell communication & 9 & 0.00512028 & 3.38 & 7.678213457 \\
\hline & GOTERM_BP_5 & GO:0043067 regulation of programmed cell death & 17 & 0.00537756 & 2.15 & 8.049070072 \\
\hline & GOTERM_BP_5 & GO:0042110 T cell activation & 7 & 0.005792794 & 4.28 & 8.644672248 \\
\hline & GOTERM_BP_5 & GO:0030100 regulation of endocytosis & 5 & 0.005977852 & 6.82 & 8.90894982 \\
\hline & GOTERM_BP_5 & GO:0048534 hemopoietic or lymphoid organ development & 11 & 0.006252573 & 2.78 & 9.299955163 \\
\hline & GOTERM_BP_5 & GO:0060348 bone development & 7 & 0.006288063 & 4.21 & 9.350352896 \\
\hline & GOTERM_BP_5 & GO:0048732 gland development & 9 & 0.006612617 & 3.24 & 9.810020407 \\
\hline & GOTERM_CC 5 & GO:0009897 external side of plasma membrane & 12 & $1.52 \mathrm{E}-04$ & 4.15 & 0.18272335 \\
\hline & GOTERM_CC_5 & GO:0044459 plasma membrane part & 36 & 0.006427642 & 1.57 & 7.483216789 \\
\hline & GOTERM_MF_5 & GO:0008009 chemokine activity & 8 & $9.58 \mathrm{E}-07$ & 14.54 & 0.001074797 \\
\hline & GOTERM_MF_5 & GO:0042379 chemokine receptor binding & 8 & $1.15 \mathrm{E}-06$ & 14.17 & 0.001294254 \\
\hline & GOTERM_MF_5 & GO:0005509 calcium ion binding & 23 & 0.004240057 & 1.89 & 4.656012939 \\
\hline & GOTERM_MF_5 & GO:0004867 serine-type endopeptidase inhibitor activity & 7 & 0.006225143 & 4.20 & 6.767217317 \\
\hline & KEGG_PATHWAY & mmu04610: Complement and coagulation cascades & 13 & $2.05 \mathrm{E}-09$ & 10.47 & $2.25 \mathrm{E}-06$ \\
\hline & KEGG_PATHWAY & mmu04060: Cytokine-cytokine receptor interaction & 19 & $5.51 \mathrm{E}-08$ & 4.70 & $6.04 \mathrm{E}-05$ \\
\hline & KEGG_PATHWAY & mmu04621:NOD-like receptor signaling pathway & 10 & $5.73 \mathrm{E}-07$ & 9.74 & $6.29 \mathrm{E}-04$ \\
\hline & KEGG_PATHWAY & mmu05322: Systemic lupus erythematosus & 9 & $2.55 \mathrm{E}-04$ & 5.28 & 0.279572774 \\
\hline & KEGG_PATHWAY & mmu04062: Chemokine signaling pathway & 11 & $7.22 \mathrm{E}-04$ & 3.65 & 0.78873003 \\
\hline & KEGG_PATHWAY & mmu04623:Cytosolic DNA-sensing pathway & 6 & 0.001932068 & 6.59 & 2.099162058 \\
\hline & KEGG_PATHWAY & mmu04640: Hematopoietic cell lineage & 7 & 0.002396325 & 5.03 & 2.597563992 \\
\hline & KEGG_PATHWAY & mmu04622:RIG-I-like receptor signaling pa & 6 & 0.004900091 & 5.33 & 5.245948393 \\
\hline & KEGG PATHWAY & mmu04620:Toll-like receptor signaling pathway & 7 & 0.005430687 & 4.27 & 5.798717014 \\
\hline \multirow{11}{*}{$\begin{array}{c}\text { Downregulated } \\
\text { genes }\end{array}$} & GOTERM_BP_5 & GU:000/00/ & 9 & 0.001563235 & 4.09 & .227697321 \\
\hline & GOTERM_BP_5 & GO:0000087 M phase of mitotic cell cycle & 9 & 0.001783037 & 4.01 & 2.537196649 \\
\hline & GOTERM_BP_5 & GO:0000279 M phase & 10 & 0.0053669 & 3.05 & 7.456716398 \\
\hline & GOTERM_BP_5 & GO:0006333 chromatin assembly or disassembly & 6 & 0.008390263 & 4.76 & 11.42610313 \\
\hline & GOTERM_BP_5 & GO:0040029 regulation of gene expression, epigenetic & 5 & 0.008523676 & 6.17 & 11.59755665 \\
\hline & GOTFRM CC 5 & G0.0005694 م & 14 & $250 \mathrm{~F}-04$ & 3.38 & (299772801 \\
\hline & GOTERM CC 5 & GO:0044427 chromosomal part & 12 & $7.18 \mathrm{E}-04$ & 3.45 & 0.858611225 \\
\hline & GOTERM CC 5 & GO:0000785 chromatin & 8 & 0.002186781 & 4.43 & 2.594803536 \\
\hline & GOTERM_CC 5 & GO:0043232 intracellular non-membrane-bounded organelle & 35 & 0.002464326 & 1.67 & 2.919682295 \\
\hline & GOTERM_CC_5 & GO:0005819 spindle & 6 & 0.006738025 & 5.03 & 7.798453797 \\
\hline & GOTERM_CC_5 & GO:0005875 microtubule associated complex & 5 & 0.009426523 & 6.01 & 10.75131957 \\
\hline
\end{tabular}




\subsection{Plasmid Maps}

AAV vectors for virus generation and subsequent IN VIVo overexpression. Plasmid $A$ was the target vector for cloning of other cDNAs, but also used for control virus (AAV-GFP) generation.

A

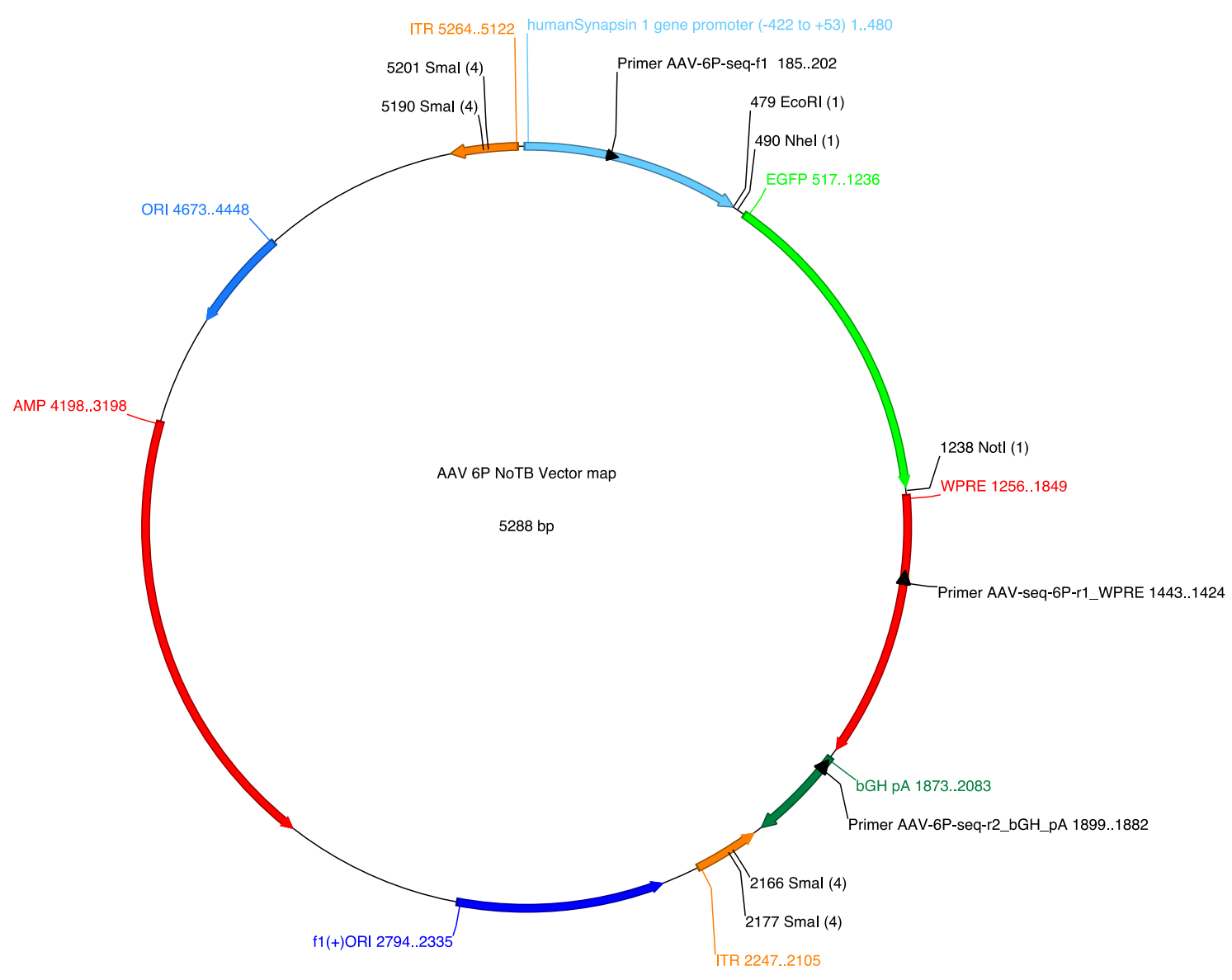


Plasmid B shows the target vector, where EGFP was exchanged for Kat2a cDNA.

B

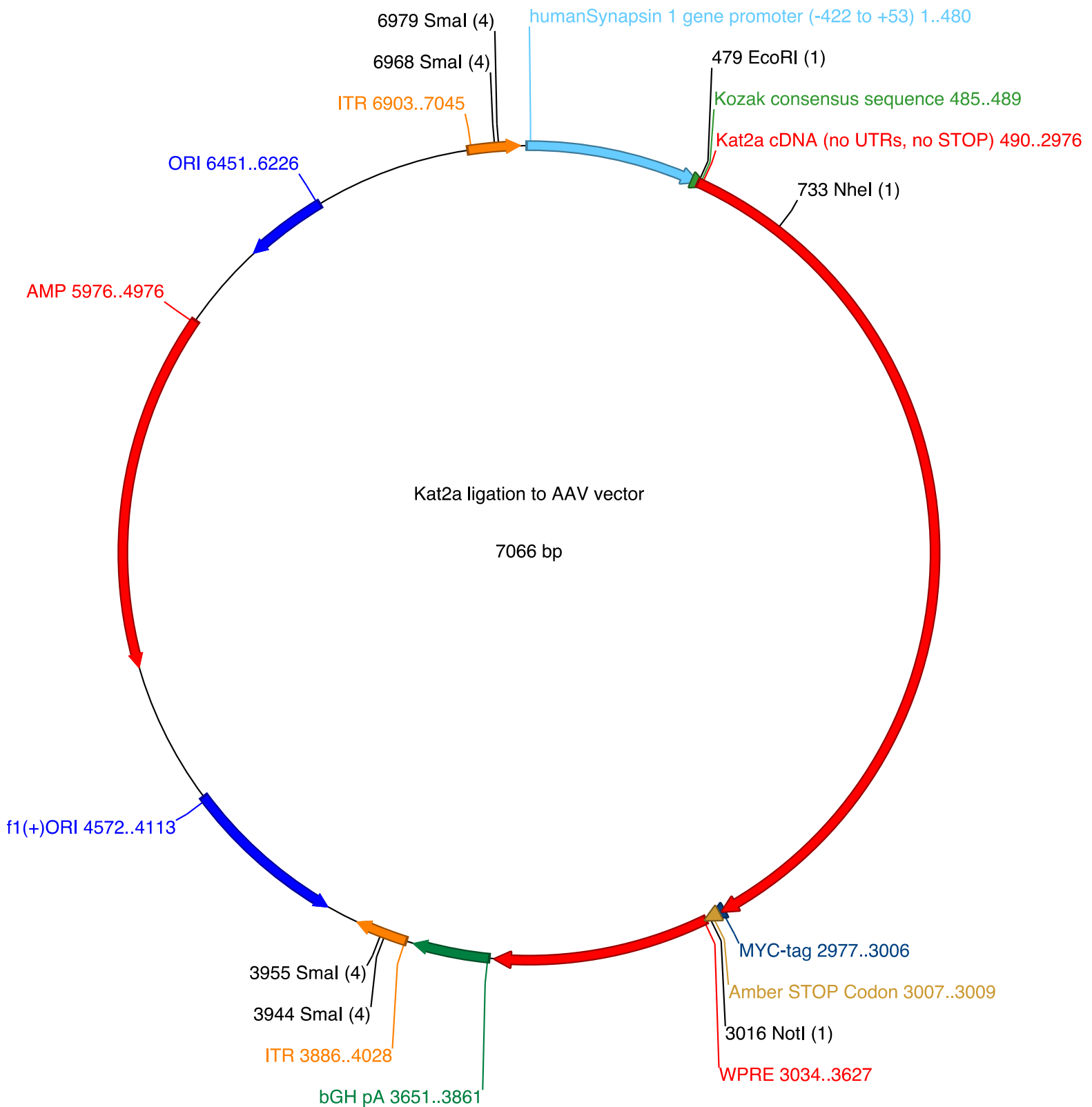




\subsection{List of Primers Used in this Thesis (in order of appearance)}

Please note that all primers were ordered from Sigma-Aldrich Custom Oligos.

Table 6-8: List of primers used in this thesis (in order of appearance)

\begin{tabular}{|c|c|c|}
\hline Name & Sequence $\left(5^{\prime} \rightarrow 3^{\prime}\right)$ & Used for \\
\hline Kat2a-GT_fwd & CACAGAGCTTCTTGGAGACC & Genotyping \\
\hline Kat2a-GT_rev & GGAGGTACAGGAATCAAGCC & Genotyping \\
\hline Kat2a-GT_rev2 & TGTAGAATGTCTGGTGGCCA & Genotyping \\
\hline Kat2a-myc-fwd & CCGGAATTCCCACCATGGCGGAACCTTCCCAGGCCC ${ }^{28}$ & Linker-PCR (2.4.3.1) \\
\hline Kat2a-myc-rev & CAGATCCTCTTCTGAGATGAGTTTTTGTTCCTTGTCGATGAGC & Linker-PCR \\
\hline Myc-STOP-Notl & ATTTGCGGCCGCTTTATCCTACAGATCCTCTTCTGAGATGAG & Linker-PCR \\
\hline Kat5-myc-fwd & CCGGAATTCCCACCATGGCGGAGGTGGGGGAG & Linker-PCR \\
\hline Kat5-rev & CAGATCCTCTTCTGAGATGAGTTTTTGTTCCCACTTTCCTCTC & Linker-PCR \\
\hline Kat7-myc-fwd & CTAGCTAGCCCACCATGCCGCGAAGGAAGAGAAATG & Linker-PCR \\
\hline Kat7-myc-rev & CAGATCCTCTTCTGAGATGAGTTTTTGTTCAGTGCCCTTGGGA & Linker-PCR \\
\hline Hdac2-myc-fwd & CCGGAATTCCCACCATGGCGTACAGTCAAGGAGG & Linker-PCR \\
\hline Hdac2-myc-rev & CAGATCCTCTTCTGAGATGAGTTTTTGTTCAGGGTTGCTGAGT & Linker-PCR \\
\hline Hdac3-myc-fwd & CCGGAATTCCCACCATGGCCAAGACCGTGGCG & Linker-PCR \\
\hline Hdac3-myc-rev & CAGATCCTCTTCTGAGATGAGTTTTTGTTCAATCTCCACATCAC & Linker-PCR \\
\hline AAV-6P-seq-f1 & САСТGCCAGCTTCAGCAC & $\begin{array}{l}\text { Sequencing of AAV } \\
\text { construct }\end{array}$ \\
\hline AAV-6P-seq-r1_WPRE & GGGCCACAACTCCTCATAAA & $\begin{array}{l}\text { Sequencing of AAV } \\
\text { construct }\end{array}$ \\
\hline AAV-6P-seq-r2_bGH_pA & TAGAAGGCACAGTCGAGG & $\begin{array}{l}\text { Sequencing of AAV } \\
\text { construct }\end{array}$ \\
\hline C4b-qRT-PCR-fwd & TCTCACAAACCCCTCGACAT & qRT-PCR (UPL \#10) \\
\hline C4b-qRT-PCR-rev & AGCATCCTGGAACACCTGAA & qRT-PCR (UPL \#10) \\
\hline Hprt-qRT-PCR-fwd & ТССТССТСAGACCGСTTTT & qRT-PCR (UPL \#95) \\
\hline Hprt-qRT-PCR-rev & ССTGGTTCATCATCGCTAATC & qRT-PCR (UPL \#95) \\
\hline Gfap-qRT-PCR-fwd & TCGAGATCGCCACCTACAG & qRT-PCR (UPL \#67) \\
\hline Gfap-qRT-PCR-rev & GTCTGTACAGGAATGGTGATGC & qRT-PCR (UPL \#67) \\
\hline
\end{tabular}

\footnotetext{
${ }^{28}$ Legend: BLACK: Restriction facilitating extension - RED: Restriction site - GREEN: Kozak consensus sequence - DARK GREEN: cDNA specific - YELLOW: MYC-tag - PURPLE: Stop-Codon
} 


\subsection{Lists of Antibodies Used in this Thesis}

\subsubsection{Primary Antibodies}

Table 6-9: List of primary antibodies used in this thesis (in alphabetical order)

\begin{tabular}{|c|c|c|c|c|}
\hline Name & Company, catalog number & $\begin{array}{l}\text { Dilution for } \\
\text { Western Blot }\end{array}$ & $\begin{array}{l}\text { Dilution for } \\
\text { IHC }\end{array}$ & $\begin{array}{l}\text { Amount used } \\
\text { for ChIP [ } \mu g]\end{array}$ \\
\hline$\beta$-Actin & Santa Cruz, sc-69879 & $1: 1,000$ & & \\
\hline $\mathrm{C} 4$ [16D2] & Abcam, ab11863 & - & 1:100 & - \\
\hline Gfap & $\begin{array}{l}\text { kind gift from Anja Schneider } \\
\text { (DZNE-G) }\end{array}$ & - & $1: 1,000$ & - \\
\hline $\mathrm{H} 1$ & Santa Cruz, sc-8030 & & - & - \\
\hline H3 & Abcam, ab1791 & & & \\
\hline H3K9ac & Merck Millipore, 07-352 & $1: 1,000$ & - & 4.5 \\
\hline H3K14ac & Merck Millipore, 07-353 & $1: 1,000$ & - & - \\
\hline $\mathrm{H} 4$ & Abcam, ab10158-100 & $1: 1,000$ & - & - \\
\hline $\mathrm{H} 4 \mathrm{~K} 12 \mathrm{ac}$ & Merck Millipore, 07-595 & $1: 1,000$ & - & - \\
\hline Hdac2 [H54] & Santa Cruz, sc-7899 & $1: 1,000$ & - & - \\
\hline Hdac3 [H99] & Santa Cruz, sc-11417 & $1: 1,000$ & - & - \\
\hline Iba1 & $\begin{array}{l}\text { kind gift from Anja Schneider } \\
\text { (DZNE-G) }\end{array}$ & $1: 1,000$ & - & - \\
\hline Kat2a (Gcn5l2) & Cell Signaling, \#3305 & $1: 1,000$ & 1:100 & - \\
\hline Map2 & SySy, 188002 & - & $1: 1,000$ & - \\
\hline MYC [9E10] & Abcam, ab32 & - & $1: 1,000$ & - \\
\hline $\begin{array}{l}\text { NeuN (Rbfox3) } \\
{[A 60]}\end{array}$ & Merck Millipore, MAB377 & - & $1: 1,000$ & - \\
\hline Plp1 & $\begin{array}{l}\text { kind gift from Anja Schneider } \\
\text { (DZNE-G) }\end{array}$ & - & $1: 1,000$ & - \\
\hline Svp & Sigma, S5768 & - & $1: 1,000$ & - \\
\hline Synpr & SySy, 102002 & - & $1: 1,000$ & - \\
\hline
\end{tabular}

\subsubsection{Secondary Antibodies}

Table 6-10: List of antibodies used in this thesis (in alphabetical order)

\begin{tabular}{cccc}
\hline Name & Company, catalog number & $\begin{array}{c}\text { Dilution for } \\
\text { Western Blot }\end{array}$ & $\begin{array}{c}\text { Dilution for } \\
\text { IHC }\end{array}$ \\
\hline Alexa 488 a-mouse & life Technologies, A11029 & - & $1: 1,000$ \\
Alexa 488 a-rabbit & life Technologies, A21206 & - & $1: 1,000$ \\
Cy3 a-rabbit & $\begin{array}{c}\text { life Technologies, A10520 } \\
\text { Jackson ImmunoResearch, } \\
\text { Cy3 a-rat }\end{array}$ & - & $1: 1,000$ \\
Li-cor IRDye 800 a-mouse & Li-cor, 926-32210 & $1: 15,000$ & $1: 1,000$ \\
Li-cor IRDye 800 a-rabbit & Li-cor, 926-32211 & $1: 15,000$ & \\
\hline
\end{tabular}




\section{Curriculum vitae}

\section{$\underline{\text { Roman Manuel Stilling }}$}

born 02.08.1984 in Beja / Portugal

\section{Education}

$10 / 2009-04 / 2013$

University of Goettingen, Germany

PhD thesis in the European Neuroscience Institute Göttingen, under supervision of Prof. Dr. André Fischer.

10/2008-09/2009 University of Goettingen, Germany

M.Sc./PhD Program at the International Max-Planck-Research School (IMPRS) Neurosciences with final examination, Master thesis waived

$10 / 2005-09 / 2008$

University of Muenster, Germany

B.Sc. in Biology (1550/1700 points)

$08 / 1995-07 / 2004$

Kopernikus Gymnasium Rheine, Germany

Graduation "Allgemeine Hochschulreife" (Qualification for university studies)

Research Projects

10/2009 - present

PhD thesis. (Prof. Dr. André Fischer)

$01 / 2009-06 / 2009$

3 lab rotations in the course of the MSc/Ph.D Program Neuroscience

$03 / 2008-08 / 2008$

Bachelor Thesis, Institute of Neuro and Behavioural Biology, University of Münster. The role of Posterior sex combs (Psc) in olfactory neuron differentiation in Drosophila melanogaster (Prof. Dr. Thomas Hummel).

$02 / 2007-08 / 2008$ Student assistant, Department of Dermatology, University of Münster (Prof. Dr. Annegret Kuhn). I worked on a project involving phototesting of Cutaneous Lupus Erythematosus, including literature research and data management.

Scholarships

$10 / 2008-08 / 2009$ Stipend of the Excellence Foundation for the Promotion of the Max Planck Society 


\section{Publications}

Kerimoglu C, Agis-Balboa R*, Kranz A*, Stilling, R*, Bahari Javan S, Benito-Garagorri E, Halder R, Burkhardt S, Stewart FA, Fischer A. Histone-Methyltransferase Mll2 (Kmt2b) is required for memory formation in mice. J Neurosci 2013 Feb 20;33(8):3452-64, *equal contribution

Ruland V, Haust M, Stilling RM, Amler S, Ruzicka T, Kuhn A. Updated analysis of standardised photoprovocation in patients with cutaneous lupus erythematosus. Arthritis Care Res (Hoboken). 2012 epub ahead of print

Zovoilis A, Agbemenyah HY, Agis-Balboa RC, Stilling RM, Edbauer D, Rao P, Farinelli L, Delalle I, Schmitt A, Falkai P, Bahari-Javan S, Burkhardt S, Sananbenesi F, Fischer A. microRNA-34C is a novel target to treat dementias. EMBO J. 2011;30(20):4299-4308.

Stilling RM, Fischer A. The role of histone acetylation in age-associated memory impairment and Alzheimer's disease. Neurobiol Learn Mem. 2011;96(1):19-26. (Review article)

Stilling RM, Fischer A. A Drosophila model for the role of epigenetics in brain function and development. Genome Biol. 2011;12(2):103. (Research highlight)

Kuczera T, Stilling RM, Hsia H-E, u. a. The anaphase promoting complex is required for memory function in mice. Learn. Mem. 2011;18(1):49-57. 